Check for updates

Cite this: Mater. Adv., 2022, 3, 19

Received 10th June 2021 Accepted 18th October 2021

DOI: 10.1039/d1ma00506e

rsc.li/materials-advances

\title{
Nitrogen-rich covalent organic frameworks: a promising class of sensory materials
}

\author{
Himanshi Bhambri, ${ }^{a}$ Sadhika Khullar, (D) ${ }^{\mathrm{b}}$ Sakshi $^{\mathrm{b}}$ and Sanjay K. Mandal (D) $\star^{\mathrm{a}}$
}

\begin{abstract}
Covalent organic frameworks (COFs) have emerged as highly crystalline porous organic materials and proven to be potential candidates for various interesting applications, such as gas adsorption and separation, catalysis, energy storage, and sensing. Fluorescence-based sensing has attracted significant attention due to its high selectivity, rapid response time, and real-time monitoring. Lately, luminescent COFs have been developed as sensing probes to detect various target molecules. In this review, we present a comprehensive account of the chronological development of the field of COFs starting from 2005 to date. We describe the design principles of COFs with a focus on various topologies reported, classification of their structural diversity based on thirty-two linkages and dimensionality, methods for their synthesis with examples, and the use of various characterization techniques. Interestingly, almost half of the COFs contain nitrogen-rich linkages. With a background in different processes involved in fluorescence sensing, we illustrate the potential applicability of nitrogen-rich COFs as sensors for numerous analytes, such as explosives, volatile organic molecules, $\mathrm{pH}$, temperature, toxic anions, metal ions and biomolecules in different media utilizing their striking features with predesigned structures and diverse functionality.
\end{abstract}

\section{Introduction}

Polymers have been known for many years and are currently indispensable in daily life. The term 'polymer' was coined by

\footnotetext{
${ }^{a}$ Department of Chemical Sciences, Indian Institute of Science Education and Research Mohali, Sector 81, Manauli PO, S.A.S. Nagar, Mohali, Punjab 140306, India.E-mail: sanjaymandal@iisermohali.ac.in

${ }^{b}$ Department of Chemistry, Dr B R Ambedkar National Institute of Technology Jalandhar, Jalandhar, Punjab 144011, India.E-mail: khullars@nitj.ac.in
}

Berzelius in 1833, which was initially muddled with the concept of 'isomers'. At that time, polymers were described as two substances having a purely compositional relationship and had nothing to do with their relative sizes, building units (organic or inorganic), structures and ability to interconvert. ${ }^{1}$ However, with the evolution of organic chemistry, the two concepts of polymerism and isomerism were set equal. Later in 1922, Straudinger introduced the term 'macromolecules' to describe large covalently bonded organic chains carrying more than 1000 atoms. $^{2}$ Organic polymers commonly have the

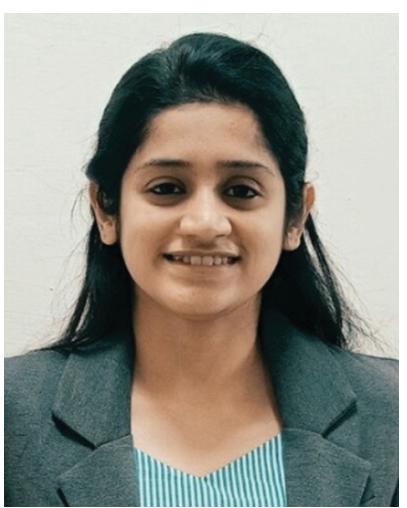

Himanshi Bhambri
Himanshi Bhambri was born in Punjab, India. She received her Bachelor of Science degree from Kurukshetra University, Kurukshetra in 2013, and Master of Science degree in Chemistry from Panjab University, Chandigarh. Currently, she is pursuing her PhD in Chemistry under the supervision of Prof. Sanjay Mandal. Her research mainly focuses on the design, synthesis, and applications of porous materials, such as metal and covalent organic frameworks (MOFs and COFs, respectively).

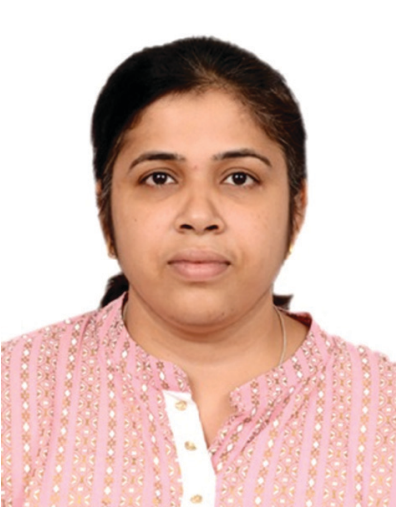

Sadhika Khullar
Dr Sadhika Khullar is currently an Assistant Professor in the Department of Chemistry at $\mathrm{Dr} B . \quad R$. Ambedkar National Institute of Technology Jalandhar. She obtained her Master's in Chemistry from Guru Nanak Dev University, Amritsar, and $P h D$ in Chemistry from IISER Mohali, Punjab, India. She is recognized for her expertise in supramolecular chemistry, catalysis, smart materials, and X-ray crystallography. 
drawback of being non-porous, one-dimensional mingled chains with compromised crystallinity and stability when exposed to heat and chemicals. There is little or no control over the synthesis of organic polymers, resulting in a lack of the above-mentioned properties. Although various attempts have been made to control the monomer sequence during the synthesis of polymers, which influences the macroscopic properties of these materials, ${ }^{3}$ the desired robustness and uniformity have not been achieved. Therefore, the two factors are of paramount importance, i.e., synthetic control of the polymer construction and choice of the precursor molecules. Consequently, covalent organic frameworks (COFs) have emerged.

COFs are highly crystalline and porous framework materials built from purely organic monomers, which exhibit exceptional robustness and stability in a wide range of organic and inorganic solvents. The synthesis of the first COF was proposed and successfully achieved by O. M. Yaghi and co-workers in 2005 using boronic acid monomer units, in which the macromolecular framework is made up from diboronic benzene molecules. ${ }^{4}$ Soon after, a huge effort was made to synthesize controlled 2D and 3D porous covalent-bonded frameworks with variable functionalities and tailorable pore shapes and sizes. ${ }^{5,6}$ Utilizing their unique and tuneable electronic and photophysical properties based on the linkages and functionalities, this class of materials has been established as potential candidates for many applications, such as gas adsorption and separation, catalysis, energy production and storage, optoelectronics, drug delivery, and sensing. ${ }^{7-11}$ However, the recent focus on their luminescent properties, which have been exploited for sensing applications, is noteworthy.

In recent years, several reviews have been published on COFs, which selectively discussed their design principle and very concise topological description, synthesis and characterization with emphasis on a particular application topic. Furthermore, only a few of these reviews included mechanistic studies for the understanding of the control over the growth of their framework and critical steps to obtain single crystal materials. Lately, a few more reviews appeared on sensing due to the above-mentioned reasons. ${ }^{12,13}$ Therefore, it is necessary to compile all these topics in an elaborate manner to connect certain types of COFs to a particular application with the underlying principles discussed to achieve a structure-property-application correlation. In this review, we attempt to discuss in the following sequence: (a) the design principle with a detailed analysis of all the topologies reported for $2 \mathrm{D}$ and $3 \mathrm{D}$ COFs, (b) their classification based on thirty-two different linkages and dimensionality, (c) mechanistic details to understand the growth of their framework, (d) their synthesis with a background on all the general methods used, and (e) their characterization via six commonly used analytical techniques. Based on this detailed background in four different sections, we then describe their luminescence properties and the processes involved in the application of nitrogen-rich COFs, which are mainly the sensing of various analytes such as explosives, pH, VOCs and small organic molecules, toxic anions, metal ions, biomolecules and temperature.

\section{Design principle}

To make a pre-designable approach and set up a regular pore framework, topological delineation must be followed precisely. The fabrication of a regular net involves the placement of the $\mathrm{C}_{n}$-reaction substrates (where ' $n$ ' is the number of reactive terminals) according to their topological connectivities and geometries, which further requires good knowledge of molecular symmetry. ${ }^{14}$ For instance, a covalent-bonded network with a honeycomb topology primarily requires a combination of planar $C_{3^{-}}$and $C_{2}$-symmetric monomers, ${ }^{4}$ whereas a square lattice combines $C_{4}$ - and $C_{2}$-symmetric units. ${ }^{15}$ The appropriate monomer/linker sets combine via strong covalently bonded linkages to reticulate into a highly ordered network under

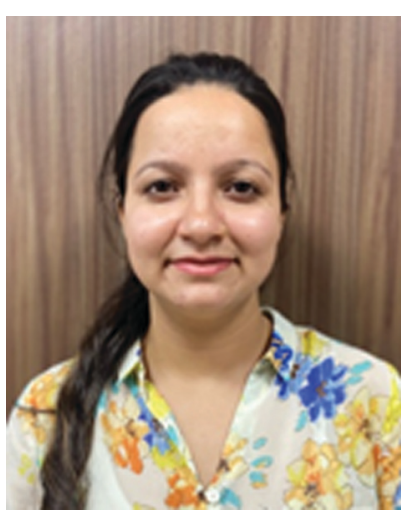

Sakshi
Sakshi is currently a PhD Fellow at Dr B R Ambedkar National Institute of Technology, Jalandhar under the supervision of Dr Sadhika Khullar. She completed her BSc (Nonmedical) in 2015 and MSc (Chemistry) in 2017, respectively, from Guru Nanak Dev University, Amritsar. Her research area includes porous materials, specifically the synthesis of coordination architectures and their application in sensing, water splitting reactions, and catalysis.

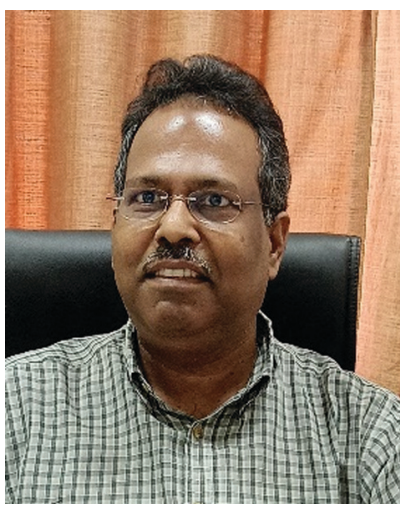

Sanjay K. Mandal
Prof. Sanjay Mandal is currently working in the department of Chemical Sciences at IISER Mohali. Prior to joining this Institute in August 2008 as one of the first faculty members, he spent over twenty years in the USA. He earned his PhD under the guidance of Professor F. Albert Cotton at Texas A\&M University. His career has been equally divided in academia and industry for over three decades. His research interests span several diverse fields involving multifunctional nanomaterials, crystal engineering, organometallic chemistry, X-ray crystallography, and Six Sigma methodologies with special emphasis on heterogeneous catalysis, and energy/environment-related topics. 
favourable conditions. Moreover, the plane of symmetry plays a vital role in the resulting dimensionality and nature of the framework. The systematic connection of planar organic monomers forms two-dimensional sheets, which lay on each other via supramolecular interactions such as $\pi-\pi$ stacking, leading to 2D COFs. AA-stacked sheets create larger pores in comparison to $\mathrm{AB}$ - and $\mathrm{ABC}$-stacked $2 \mathrm{D}$ sheets. Conversely, for an increase in the dimensionality of the framework, non-planar moieties are the appropriate choice, which yield either interpenetrated or non-interpenetrated $3 \mathrm{D}$ frameworks. ${ }^{16}$

\subsection{Topologies of $2 \mathrm{D}$ COFs}

The known topological designs for two-dimensional COFs include hcb (honeycomb), hxl (hexagonal), sql (square lattice), kgm (kagome), fxt, kgd (kagome-dual), fes, cpi, bex, mtf, htb and tth networks formed from $C_{1}, C_{2}, C_{3}, C_{4}$ and $C_{6}$-symmetric and asymmetric building units (Fig. 1). These topologies are elaborated as follows.

hcb topology. This topology is very common in 2D COFs and devised from various combinations of $C_{1}$-asymmetric and $C_{2}$ - and $C_{3}$-symmetric monomers. Primarily, the self-condensation of linear $C_{2}$-symmetric moieties, mostly having nitrile and

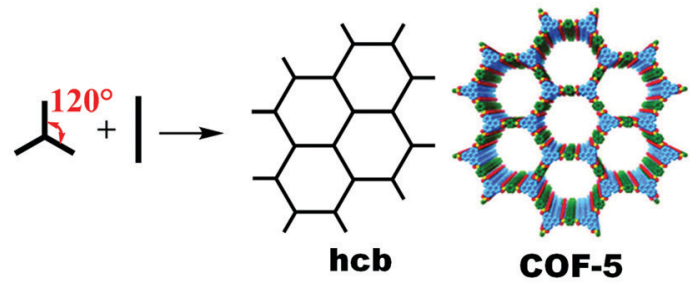
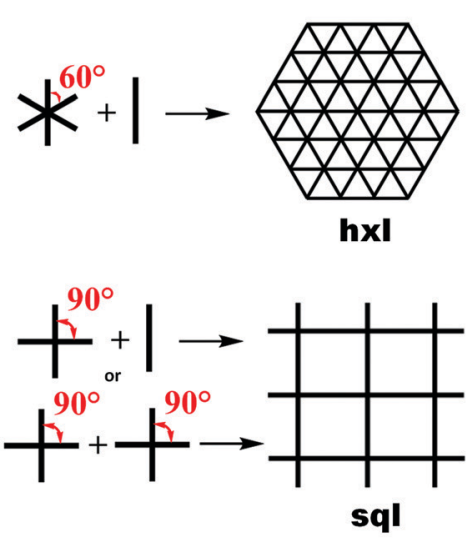

ICOF-1

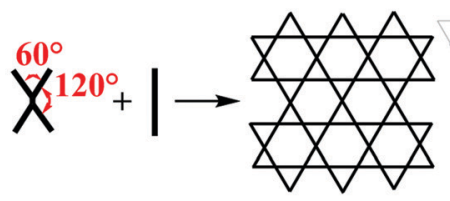

kgm
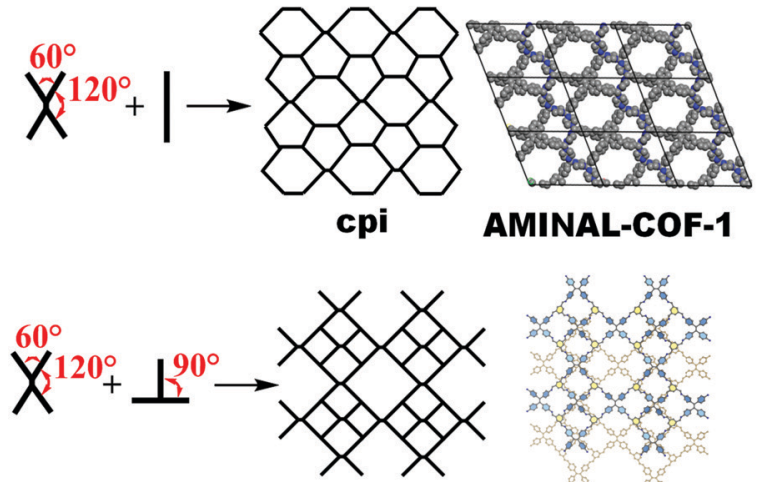

mtf

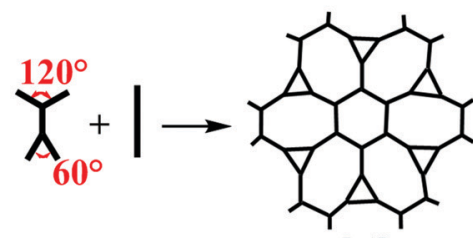

htb

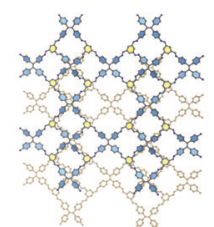

COF-432

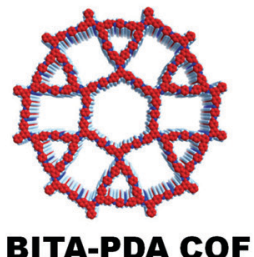

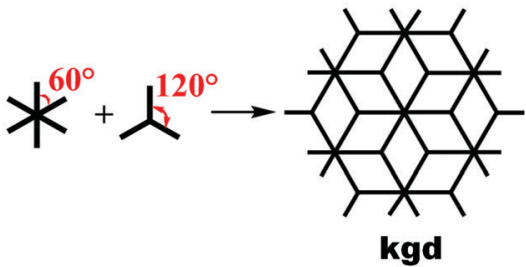
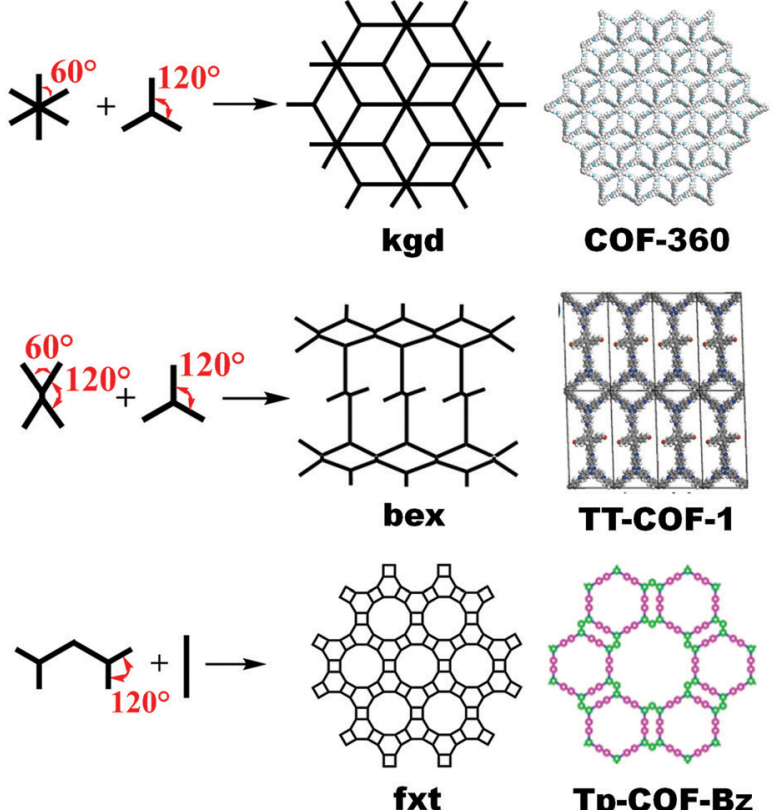

Tp-COF-Bz

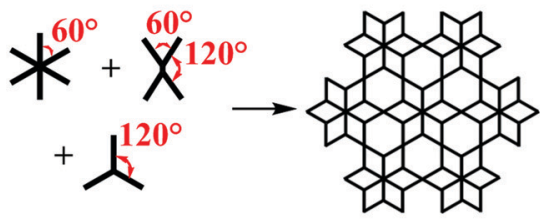

tth

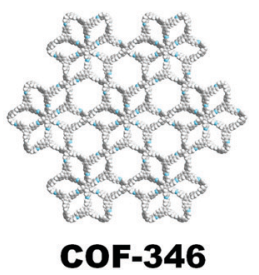

Fig. 1 Topological design for 2D COFs guided by suitable symmetries and angularity of their planar building blocks. 
boronic acid functionalities, develops hexagonal pores, forming triazine and boroxine anhydride-based frameworks, respectively. ${ }^{4,17-19}$ COFs with triazine-based linkages are also called covalent triazine frameworks (CTFs). CTF-1 consists of a terephthalonitrile unit (which is linear, planar and possesses 2 -fold symmetry), yielding edge-connected hexagons with the triazine core located at the vertices and the aromatic strut on the edges via self-condensation. ${ }^{20}$ Triazine-based frameworks knitted from $C_{2}$-asymmetric monomers are also known. ${ }^{21}$ The same topology is obtained with the $\left[C_{3}+C_{2}\right]$ combination having hexagonal pores within the framework. Depending on the stacking of the $2 \mathrm{D}$ sheets, hcb is comprised of the gra (staggered stacking) and bnn (eclipsed stacking) topologies. ${ }^{4,22}$ In addition, the combination of two planar trigonal monomers $\left[C_{3}+C_{3}\right]$ weaves a honeycomb net with similar pores. One example is the microporous COF-6 synthesized via the polycondensation reaction between 2,3,6,7,10,11-hexahydroxytriphenylene (HHTP) and 1,3,5-benzenetriboronic acid (BTBA) $\left(C_{3}\right.$-units). ${ }^{23}$ A unique six-fold interpenetrated hcb topology framework was obtained via the Schiff base reaction between tri(4-formylphenoxy)cyanurate (TFPC) and $\alpha$-aminonaphthalene, offering a starshaped channel. ${ }^{24}$

hxl topology. Another topology knitting the 2D framework is the hexagonal topology formed via the two-component condensation reaction between $C_{6}$ - and $C_{2}$-symmetric functionalities having a $\sigma_{\mathrm{h}}$ plane, where triangular micropores are obtained. ${ }^{25}$ The propellershaped hexabenzocoronene bearing six amino groups $\left(\left[\mathrm{NH}_{2}\right]_{6} \mathrm{HBC}\right)$ undergoes Schiff-base condensation reaction with a linear terephthaldehyde unit $\left[C_{6}+C_{2}\right]$ and stitches HBC-COF with supermicropores $(1.2 \mathrm{~nm}){ }^{26} \mathrm{HEX}-\mathrm{COF}$ is another example of the hxl topology, which is fabricated using the propeller-like hexaphenylbenzene core with azine linkages.

kgm topology. The kagome topology is also called hexadeltille (having hexagonal and trigonal tilling), which possesses triangular micropores and hexagonal mesopores. For the formation of a periodically arranged dual-pore kgm net, the planar $\left[C_{4}+C_{2}\right]$ symmetric building units are combined. ${ }^{27,28}$ An appropriate example is the imine-linked binary COF in which the four-fold $D_{2 \mathrm{~h}}$ symmetric ETTA $\left(4,4^{\prime}, 4^{\prime \prime}, 4^{\prime \prime \prime}\right.$-ethene(1,1,2,2tetrayl)-tetraaniline) locks with the two-fold linear terephthaldehyde to give a kagome network. The TPE-Ph COF, which was reported in 2016, exhibits a dual-pore kagome lattice when stacked in the AA-layer having tetraphenylethene units on its vertices. ${ }^{29}$ Furthermore, rhombic structures of the kgm topology are also formed, weaving a single-type pore with a $\left[C_{4}+C_{2}\right]$ combination. $^{30,31}$

kgd topology. The kagome-dual topology represents rhombille tilling, which is a tessellation of identical rhombi (looks like diamonds) with inner angles of $60^{\circ}$ and $120^{\circ} .{ }^{32}$ The synthesis of HAT-NTBCA-COF from the heterodromous orientation of imine linkages formed by fusing $\left[C_{6}+C_{3}\right]$ symmetric units, i.e., propeller-like hexaamino-hexaazatriphenylene $\left(\left[\mathrm{NH}_{2}\right]_{6} \mathrm{HAT}\right)$ and triangular symmetric linker $4^{\prime}, 4^{\prime \prime}, 4^{\prime \prime \prime}$-nitrilotris([1,1'-biphenyl]4-carboxaldehyde) (NTBA), gives a dual kagome net. ${ }^{33}$ Likewise, the $\left[C_{6}+C_{3}\right]$ combination of propeller-like hexaformyl-phenylbenzene (HFPB) and 1,3,5-tris( $p$-aminophenyl)benzene (TAPB) tailors a single-pore COF-360 framework. $^{34}$ sql topology. The combination of either $\left[C_{4}+C_{2}\right]$ or $\left[C_{4}+C_{4}\right]$ symmetric reaction substrates confers a uni-pore square lattice topology. The tetra-functional square planar $C_{4}$ unit phathalocyanine tetra(acetonide) (Pc) preferably combines with $C_{2}$ symmetric diboronic acid to stitch an upright square lattice in the Pc-PBBA COF. ${ }^{15}$ Likewise, the one-component synthesis of an azodioxylinked POR-COF from tetrakis(arylhydroxylamine)porphyrin under a protected environment creates a square lattice with the porphyrin unit covering the vertices and azodioxy-linkages located at the edges. ${ }^{35}$

fxt topology. This is a unique topology in which ternary-pore COFs are designed via the mixture of a bent $C_{4}$ unit and a linear $C_{2}$ symmetric unit. Notably, the $\mathrm{V}$-shape of the four-fold reaction substrate is essential in developing the connectivity to obtain a three-pore framework. TP-COF-BZ and TP-COF-DAB are two examples of the fxt topology utilizing terphenyl-tetracarbaldehyde with benzidine and 1,4-diaminobenzine, respectively. ${ }^{36}$

fes topology. Another rare topology in $2 \mathrm{D}$ COF materials forming a semi-regular net with one type of vertex but more than one type of face. For example, the spiroborate-linked ICOF-1 and ICOF-2 obtained via the condensation of $\mathrm{B}(\mathrm{OMe})_{3}$ with a $C_{4}$-symmetric polyol moiety create the fes topology with dual pores. ${ }^{37}$

cpi topology. The combination of a rectangular $C_{4}$-functionalized core with a linear $C_{2}$-symmetric ditopic core tailors the cpi net. The planar monomers usually fabricate the kgm topology and only one case is reported for the cpi net. Aminal-COF-1 and aminal-COF-2 were synthesized in 2019 via the condensation of linear piperazine and $D_{2 \mathrm{~h}}$-symmetric tetraldehyde units. ${ }^{38}$

bex topology. When a triangular $C_{3}$ symmetric core unites with a rectangular $C_{4}$ unit, the combination yields a dual-pore bex topology with two distinct vertex moieties. Examples of this are PT- and PY-COFs, which topologically connect pyrene-tetraniline (P) along with triazine tribenzaldehyde (T) and benzene tripicolinaldehyde (Y), respectively, as $\left[C_{4}+C_{3}\right]$ to frame a small rectangular and a large hexagonal pore. ${ }^{39}$ Likewise, TT-COF-1 and TT-COF-2 were tailored using a TPE-core rectangular $C_{4}$ with a $C_{3}$ unit.

mtf topology. This unprecedented net uses the rectangular $C_{4}$ symmetric unit with a $C_{3}$ symmetric fragment to form a voided square grid-type topology, viz., mtf. COF-432 was reportedly knitted from $4,4^{\prime}, 4^{\prime \prime}, 4^{\prime \prime \prime}$-ethene(1,1,2,2-tetrayl)tetraaniline (ETTA) and 1,3,5-triformylbenzene (TFB), where in the conformation of $\mathrm{TFB}$, the two formyl groups form a $90^{\circ}$ angle with the third formyl group. ${ }^{40}$ This net is similar to the sql topology with the removal of 1/8 4-c nodes, giving (3, 4, 4)-connectivity.

htb topology. The newly designed htb topology can be represented by a hexagonal tungsten bronze diagram, which utilizes a uniquely designed building block to tessellate the network. The $C_{4}$-functionalized monomer must have angles of $\sim 60^{\circ}, 90^{\circ}, 90^{\circ}$ and $120^{\circ}$ to meet the connectivity requirement, an example of which was reported recently. The BITA-PDA COF uses benzimidazole-based tetraaldehyde (BITA) with a $C_{2}$-symmetric linear phenylenediamine linker to knit the ternary-pore framework. ${ }^{41}$

tth topology. This distinctive topology tessellates the 2D COFs synthesized from three-component reactions, incorporating 
complexity into their structure. Therefore, careful choice of the organic building units with an appropriate linker length and angle between the two functionalities of the same unit is necessary. Considering this, COF-346 was synthesized from a hexatopic (hexaaminophenylbenzene (HAPB)), a tetratopic (tetrakis(4-aminophenyl)ethane (ETTA)) and a tritopic (1,3,5tri( $p$-formylphenyl)benzene (TFPB)) linker having the angles of $60^{\circ}, 60^{\circ}$ and $120^{\circ}$, respectively, which formed a strain-free th framework. ${ }^{34}$ On the contrary, if the tetratopic linker ETTA is replaced with either a porphyrin-tetraamine (having $90^{\circ}$ angle) or a tetrakis(4-aminophenyl)pyrene (length $11.3 \AA$ ), the topology design fails to give the desired COF. This is attributed to the higher angle of the porphyrin linker and length of the pyrenebased linker in comparison to ETTA and HAPB (ca. 7.1 ̊), which prevents the formation of a closed ring.

\subsection{Topologies of 3D COFs}

All the above-mentioned combinations with an appropriate planar geometry of organic monomers and suitable topological connectivities give rise to stacked 2D COF sheets. To increase the dimensionality of the framework, a deviation from planarity and implementation of geometric constraints over the building units are prominent choices. ${ }^{42}$ There are 15 topologies (Fig. 2) known thus far, in which 3D COFs are tessellated, six of which use monomers with a $T_{\mathrm{d}}$ geometry, for instance, dia (diamond net), ctn (cubic- $\mathrm{C}_{3} \mathrm{~N}_{4}$ ), bor (borcaite net), pts (platinum sulfide) net, ljh (Luojia Hill), lon (lonsdaleite) and rra. A few reports have been found in which the planar building units connect in such a way that they knit three-dimensional COFs, which are srs $\left(\mathrm{SrSi}_{2}\right.$ net), ffc, tbo (twisted boracite) and fjh. The former utilizes the $\left[C_{3}+C_{3}\right]$ combination, whereas the other three amalgamates the triangular $C_{3}$ unit with a $C_{4}$ symmetric monomer unit with varying geometry (rectangular or square planar). An expansion in the topological library of 3D COFs is seen when high connectivity building units $\left(C_{6}\right.$ and $\left.C_{8}\right)$ have been reported to give stp, acs, ceq and bcu (body-centered cubic) nets. Various examples of 3D COFs with different topological designs are elaborated as follows.

dia topology. A very common topology for $3 \mathrm{D}$ networks is attributed to the diamond net forged from the combination of a tetrahedral-shaped $C_{4}$ building unit and a linear $C_{2}$ monomer or another $T_{\mathrm{d}}$ node. ${ }^{43}$ For example, the extended 3D network of COF-300 was synthesized using tetra-(4-anilyl)methane (TAM), which is a tetrahedral unit, and terephthaldehyde via a twocomponent polycondensation reaction, leading to the formation of the dia topology. ${ }^{6}$ COF-300 crystallizes in the body-centered
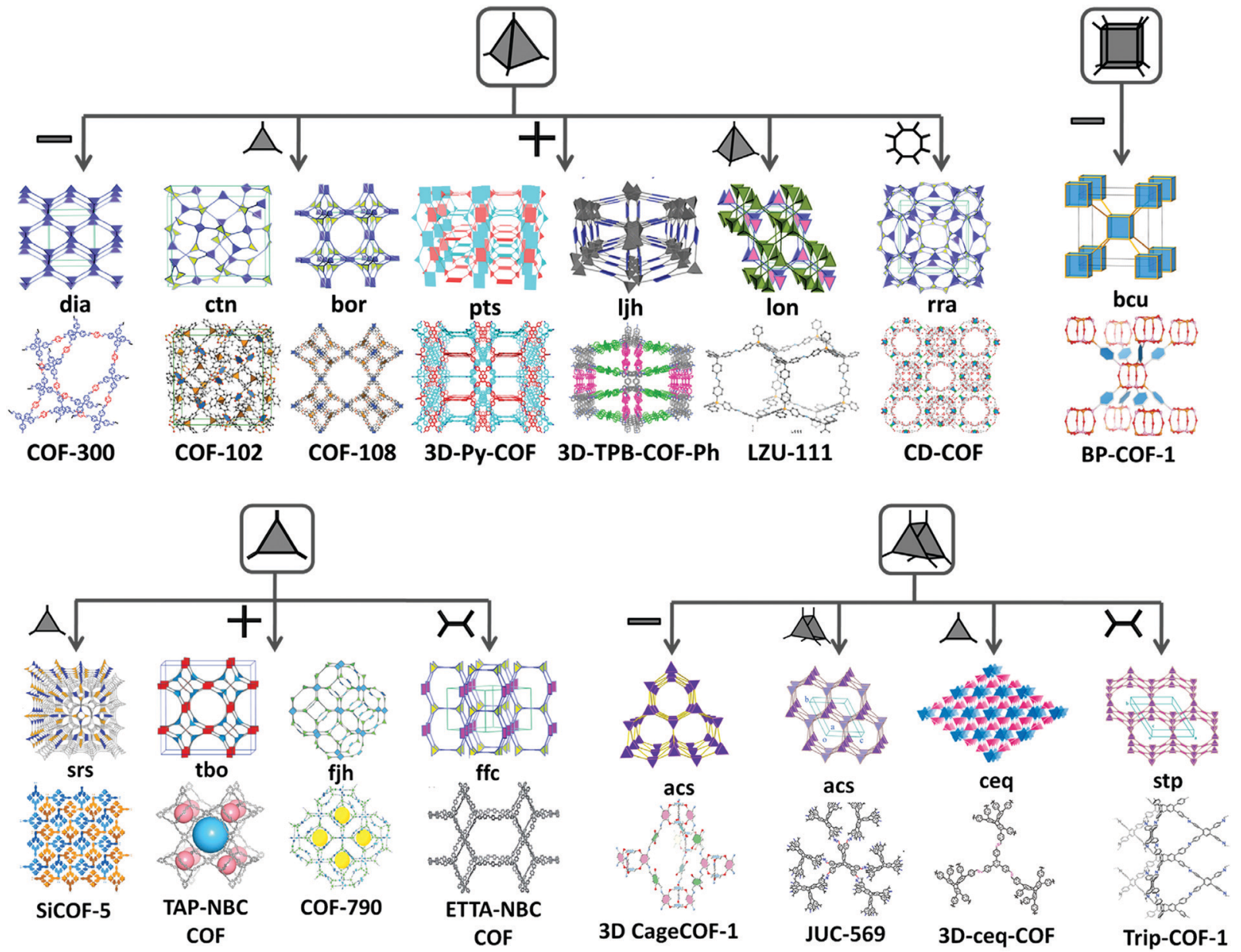

Fig. 2 Topological designs for 3D COFs guided by combining symmetrical, planar and non-planar building blocks. 
tetragonal space group with a dia-c7 net with seven-fold interpenetration. However, the first non-interpenetrated dia net was found in PI-COF-4, in which the imidization of tetraaminoadmantane (TAA) (a smaller-sized $T_{\mathrm{d}}$ monomer, ca. $3.1 \AA$ ) and pyromellitic dianhydride (PMDA) occurs. ${ }^{44}$ Steric factors play a role in hindering the interpenetration here.

ctn and bor topology. The $\left[C_{4}+C_{3}\right]$ combination of the reaction substrates gives both ctn and bor nets, which differ only in their topological densities and pore size inside the framework. The topological density is "the number of vertices per unit volume for the given embedding". The Bor net is observed to be topologically less dense than the ctn net, with the latter being more strain free and possessing smaller pores. COF-105 and COF-108 are unified in the ctn and bor topology, giving lower densities of 0.41 and $0.17 \mathrm{~g} \mathrm{~cm}^{-3}$, respectively. ${ }^{45}$ This is attributed to the different synthetic units in both COFs, given that COF-108 is formed by cross-condensation of tetra(4dihydroxyborylphenyl)methane (TBPM) with a triangular hexahydroxytriphenylene (HHTP) unit in comparison to COF-108 formed by cross-condensation of the silane analog of TBPM and HHTP. Thus, the linker geometry does not explain the topological difference between the two.

pts topology. In the platinum sulphide-like net, a tetrahedral $C_{4}$-symmetric unit, which replaces the sulphide ions, combines with a square planar $C_{4}$-unit at the location of the platinum nodes to give the underlying pts topology design. This is more likely to exist in an interpenetrated form. 3D-Py-COF obtained from the $\left[C_{4}+C_{4}\right]$ imine condensation reaction between tetra $(p$ aminophenyl)methane (TAPM) and tetrakis(4-formylphenyl)pyrene (TFFPy) affords a two-fold interpenetrated pts net with vague $\pi-\pi$ interactions. $^{46}$

ljh topology. This is an unusual topology, which was reported recently by introducing steric factors in the monomers of the pts net. The very common combination of $\left[T_{\mathrm{d}}+C_{4}\right]$ using tetra( $p$-aminophenyl)methane (TAPM) and 1,2,4,5-tetrakis(4-formylphenyl)-3,6-diphenylbenzene (TPB-Ph) formed a rare unprecedented self-penetrated ljh net. ${ }^{47}$

lon topology. A rare topology representing the lonsdaleite network is woven using two $T_{\mathrm{d}}$ units. The lon topology usually competes with the dia net, which is quite commonly knitted with the $\left[T_{\mathrm{d}}+T_{\mathrm{d}}\right]$ combination. In 2018, single crystals of LZU111 were obtained via the imine condensation of tetra-(4anilyl)methane (TAM) and tetra(4-formylphenyl)silane (TFS), which crystallize in a chiral $P 6_{5}$ space group with a three-fold interpenetrated lon-b-c3 topology. ${ }^{6}$ Surprisingly, despite the use of achiral monomers, the $6_{5}$ screw axis was found to be generated from an interpenetration within the network.

rra topology. With the idea of incorporating pliable building units to diversify the topological domain of covalently linked porous organic networks, a $\gamma$-cyclodextrin monomer was used to form a 3D anionic CD-COF with the rra net. ${ }^{48}$ The topology was constructed by combining a 4-c $T_{\mathrm{d}}$ anionic borate node with eight hydroxyl groups of $\gamma$-cyclodextrin via the formation of spiroborate linkages.

srs topology. There are a few network designs that are constructed from planar building units, but higher dimensionality is achieved. One of these nets is srs, representing a strontium silicide framework knitted from a $\left[C_{3}+C_{3}\right]$ monomer combination. The first COF with the srs net is the anionic SiCOF-5 synthesized from the condensation of a catecholate building unit (HHTP) and tetramethoxysilane (TMOS) using the reversible Si-O chemistry. ${ }^{49}$ Here, the TMOS unit acts as a silicon source, which in situ forms the dianionic octahedral $\left[\mathrm{SiO}_{6}\right]^{2-}$ building unit, and hence increases the dimensionality of the whole framework. The anions are stabilized by alkali metal ions.

ffc topology. Another unusual 3D topology formed from planar monomeric units is ffc, which is obtained by mixing a rectangular unit and a triangular unit. The $\left[C_{4}+C_{3}\right]$ imine condensation of ETTA with NBC yields ETTA-NBC COF (named COF-1 in the original paper), which contains two independent $(3,4)$-coordinated nets interwoven with each other to increase the dimensionality. ${ }^{50}$

tbo topology. The tbo net represents a twisted boracite, which differs from the ffc net with the geometry of the $C_{4}$ symmetric unit. It uses a 4-connected square planar monomer together with a 3-connected triangular unit to yield a noninterpenetrated 3D framework with three different polyhedron sharing faces when closely packed. The truncated tetrahedron polyhedron cage shares the trigonal face with the truncated cube, which further shares an octagon face with the truncated cuboctahedron. A porphyrin-based square planar amine unit $\left(\mathrm{H}_{2} \mathrm{TAP}\right)$ underwent $\left[C_{4}+C_{3}\right]$ imine condensation with the tritopic nitrilotris(biphenyl)carbaldehyde (NBC) unit to form the TAP-NBC COF (named COF-1 in the original paper). ${ }^{51}$

fjh topology. The combination of trigonal-planar and squareplanar moieties connected at the dihedral angles of $\sim 75-90^{\circ}$ resulted in an fjh net for COF-790, which has rarely been reported. For its synthesis, a trimethyl substituted tris(4formylphenyl)benzene (TTFB) was chosen with 4-c ETTA, which provided an appropriate dihedral angle due to the position of the methyl group. ${ }^{52}$ A single crystal of COF-790 was obtained with the molecular formula $\left[(\text { TTFB })_{4}(\text { ETTA })_{3}\right]_{\text {imine, }}$, which crystallizes in the orthorhombic Iba2 (No. 45) space group. It has been investigated that the additional substitution of methyl on TFPB contributes to the crystallization in the fjh topology.

stp topology. Recently, JUC-564 COF was synthesized using a $D_{3 \mathrm{~h}}$-symmetric trigonal prism unit with a triptycene-based core as a 6-c node with a 4 -c $D_{2 \mathrm{~h}}$ square-planar monomer. ${ }^{53}$ The unique link angle of $60^{\circ}$ and $120^{\circ}$ in the respective linkers yielded the novel stp topology for the low-density large-pore COF. A few months later, two other triptycene-centered COFs, Trip-COF 1 and Trip-COF2, were reported with an stp net via the $\left[C_{6}+C_{4}\right]$ combination using rigid hexagonal hexakis(4formylphenyl)triptycene (HFPTP) with tetraamino benzene and pyrene units, respectively. ${ }^{54}$

acs topology. The combination of a high connected trigonal prism node with a linear ditopic unit weaves the symmetryguided design for the acs topology. This rare net was reported when the 3D-cage COF-1 was synthesized from a $D_{3 \mathrm{~h}}$-symmetric cage-6- $\mathrm{NH}_{2}$ unit and linear 2,5-dihydroxyterephthaldehyde (DHTPA). ${ }^{55}$ Reversible structural transformation was noted between the small-pore and large-pore with the loading of DMF, although the topology remained intact. 
ceq topology. Another rarely reported topology with a high coordination number is ceq. 3D-ceq net was constructed using a rigid $D_{3 \mathrm{~h}}$-symmetric hexaformyl triptycene moiety oriented at $120^{\circ}$ at vertical positions with a substituted triangular planar organic strut. $^{56}$ Therefore, the connectivity of the 6-c trigonal prismatic unit with three different planar monomers, i.e., square planar, triangular and linear, yields stp, ceq and acs nets, respectively.

bcu topology. This indicates body-centered cubic connectivity from the combination of an 8-coordinated core having a link angle of $\sim 70.5^{\circ}$ with 2-c linear units. A polycubane BP-COF-1 was reported in which eight borophosphonic acid units join to give BP-cubes with B-O-P linkages. ${ }^{57}$ The $\mathrm{sp}^{3}$-hybridised B- and P-atoms located at the cube vertices connect the next eight BP-cubes through phenyl linkers, yielding a polycubane framework with the bcu topology.

\section{Classification of COFs}

\subsection{Based on their linkages}

There are numerous linkages used for constructing COFs with high crystallinity, stability towards heat, chemicals and water, and enormous surface areas with sizeable pores and functionalization (Fig. 3). Topological diversity can help explain the use of these linkages. COFs with good crystallinity, exceptional stability and multiple functionalization are challenging to synthesize given that control of their growth is required (longrange order), but functionalization results in the loss of this control. Furthermore, thermal, chemical and hydrolytic stability are other challenges to overcome within a single framework. An attempt to combine different synthetic strategies to engineer the desired COFs was envisioned by Lotsch and co-workers last year. ${ }^{58}$ It is known that by employing dynamic covalent chemistry, one can control the covalent bond formation rate. Accordingly, introducing reversible linkages within the framework of COFs causes them to be more crystalline, robust and resistant upon exposure to heat; however, their stability towards moisture and several organic and inorganic chemicals is sacrificed. For instance, the first thermally stable and ordered boroxine and boronic ester-linked COFs, namely, COF-1 and COF-5, disintegrate in the presence of water following Le Chatelier's principle. Water, as the by-product, shifts the reaction to the backward direction, limiting or preventing the formation of a COF. Recent studies have shown that to maintain the 'error-correction' and 'proofreading' during COF synthesis, a calculated amount of $\mathrm{H}_{2} \mathrm{O}$ ( $\sim 15$ equiv.) is required to get highly crystalline COF-5 with controlled crystallite sizes. ${ }^{5}$ The COF would not be formed when water content is increased to 20 equiv. However, functionalizing the linkers with alkyl chains in boron-containing COFs can provide resistance to aqueous medium to some extent. ${ }^{59}$

Further attempts have been made to increase the stability of COFs in thermal, chemical and humid environments by incorporating N-containing bonds within their framework. The various linkages containing a nitrogen heteroatom include triazine, imine, heptazine, hydrazone, aminal, phenazine, azine, squarine, azidoxy, anhydride, indoimine, nitrile-substituted olefin, amide, viologen, urea, and pyrimidazole, as shown Fig. 3. These $\mathrm{N}$-containing linkages enrich the framework with robust reversible bonds and provide protection against different organic and inorganic chemicals and moisture. Based on a detailed study and literature comparison, it can be perceived that the COFs enriched with $\mathrm{N}$-centres are highly crystalline, possess great stability and can tolerate functionalization of their framework compared to boron-based linkages (Table 1).

Linkages such as borazine, borosilicate and polycubane were obtained by introducing $\mathrm{N}, \mathrm{Si}$ and $\mathrm{P}$ atoms in boron-rich frameworks, endowing the frameworks with exceptional crystallinity, but their chemical and hydrolytic stabilities are still lacking. ${ }^{57,60,61}$ Some oxygen-containing linkages have also contributed to the stability, i.e., dioxin, ester and silicate-based, examples of which are shown in Table $1 .^{49,62,63}$ To explore more traits, the chemistry of knitting a framework with two different linkages was practiced. The N-rich imine linkage was intermixed with boroxine (or boronic ester) and $\beta$-ketoenamine to enhance the crystallinity and stability. ${ }^{64,65}$ Furthermore, the boroxine and hydrazone linkages were taken in a single framework to overcome the chemical stability limitation and allow selective hydrolysis in the framework. ${ }^{66}$ These COFs are known as double-stage COFs.

Lately, it has been found that incorporating reversibility during the synthesis of COFs is not the only route to attain all the required properties in one frame. Hence, combining reversible covalent bond-forming reactions with irreversible non-covalent interactions has gathered increasing attention to solve the above-mentioned problem. Remarkably, stable materials with intact crystallinity in harsh acidic, basic and aqueous conditions have been synthesized using this strategy, which can also withstand chemical functionalities tied up on the framework. These linkages are $\beta$-ketoenamine, ${ }^{67}$ bis(imidazole), ${ }^{68}$ benzo-bis(oxazole) ${ }^{69}$ and thiazole ${ }^{70}$ (Fig. 4). In these types of linkages, the pre-orientation of the building units to induce order via reversible linkages provides crystallinity and irreversible locking of the bonds to induce stability in the framework synergistically.

Nevertheless, the fabrication of ordered COFs solely using irreversible reactions is a very cumbersome process. However, by limiting the flexibility and reducing the degree of freedom in the building units, a kinetically controlled but crystalline framework can be obtained. One example of this was reported by Liu et al. in 2017, where a highly ordered 2D-CAP COF from the exceptionally rigid 2,7,13,18-tetrabromodibenzo[a,c]dibenzo $[5,6: 7,8]$ quinoxalino[2,3-i]phenazine (2-TBQP) building block was successfully engineered. ${ }^{71}$ In addition to the 2D framework, an energetically less favourable 1D-CAP was formed. The 2D polymer was formed via metal-surface-mediated polymerization at an elevated temperature of $\sim 250{ }^{\circ} \mathrm{C}$, where the debromination of 2-TBQP and aryl-aryl coupling reaction occurred. A similar example of an irreversible $\mathrm{C}-\mathrm{C}$ coupling reaction was reported the next year, in 2018, forming highly conjugated 2D aromatic polymers, viz., CAP-1 and CAP-2, at high temperature and under an inert atmosphere (Fig. 5). ${ }^{72} \mathrm{~A}$ few other COFs synthesized using irreversible Ullmann, Suzuki-Glaser ${ }^{73,74}$ and Horner-Wadsworth-Emmons reactions ${ }^{75}$ were also reported. 


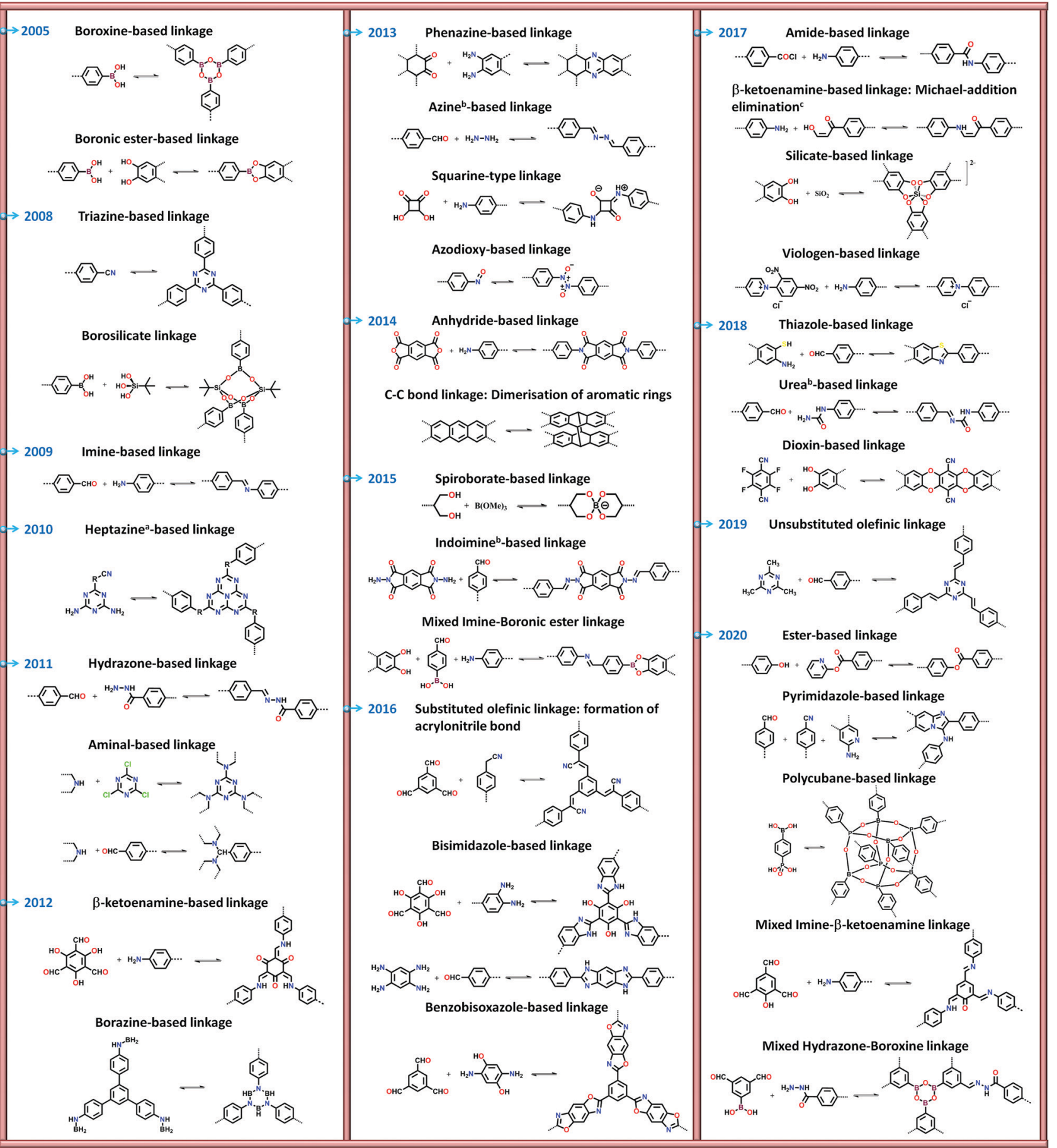

Fig. 3 Types of linkages in COFs with a timeline for their discovery and progress. ${ }^{a}$ Subclass of imine linkages, ${ }^{b}$ subclass of hydrazone linkages and ${ }^{c}$ falls under $\beta$-ketoenamine linkage.

\subsection{Based on their dimensionality}

Considering their dimensionality, COFs are categorized as $2 \mathrm{D}$ and 3D COFs. As explained earlier in Section 2, following the topological delineation of 2D COFs, planar building units from the diverse list of monomers are used, whereas deviation from planarity is one of the requirements to yield 3D frameworks. In this section, we focus on the chemistry involved in the formation of $2 \mathrm{D}$ and $3 \mathrm{D}$ frameworks using covalently bonded organic units.
3.2.1 2D COFs. Upon closely monitoring the mechanism through which two-dimensional covalent organic frameworks are formed in the reaction, two forces have been found to play an essential role as follows: (1) the reversible covalent bond formation among the organic monomers and (2) the supramolecular interactions formed among the $2 \mathrm{D}$ sheets. The use of dynamic covalent chemistry involves reversibility in the organic reaction by making and breaking covalent bonds, which directs 


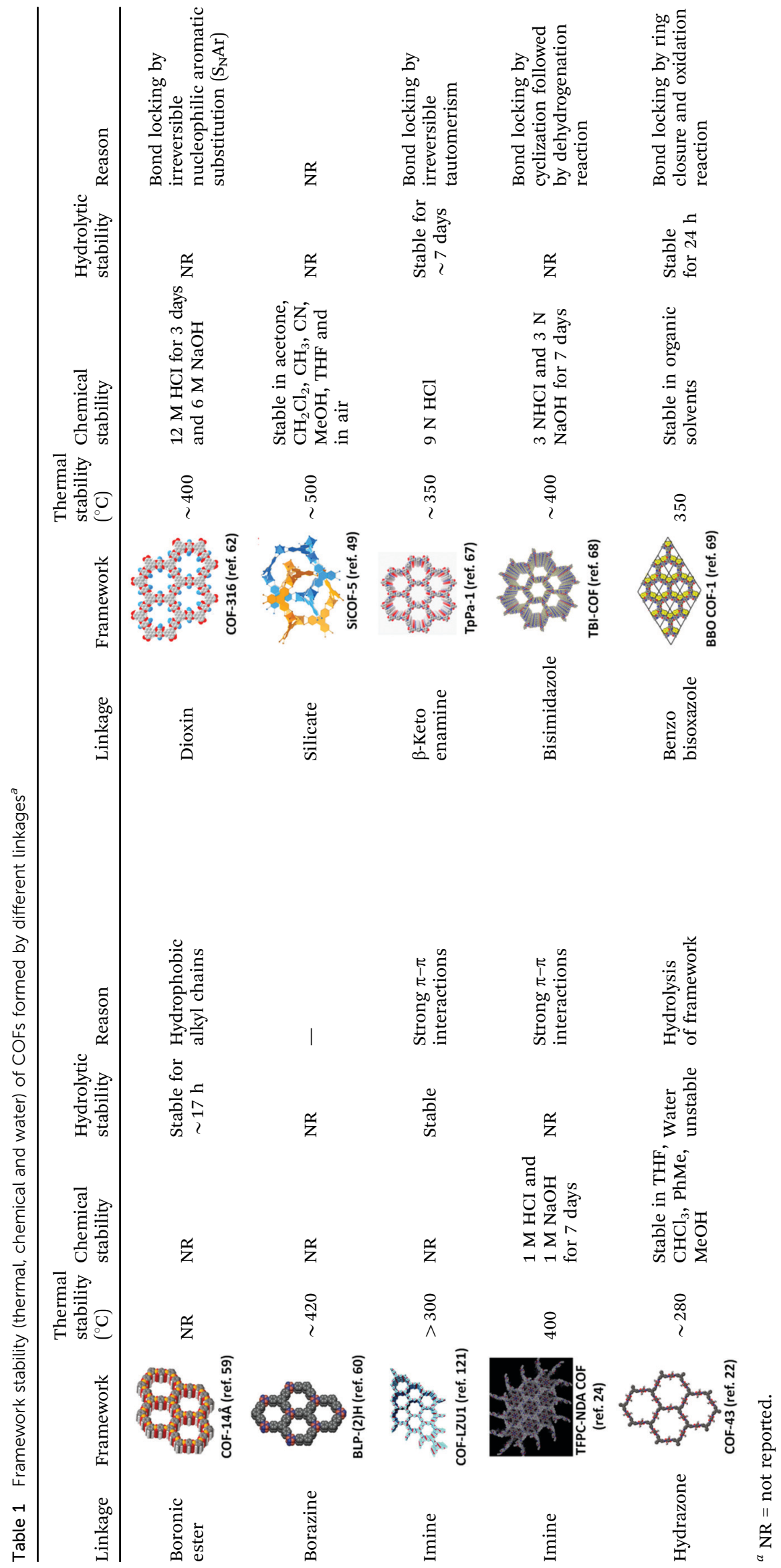




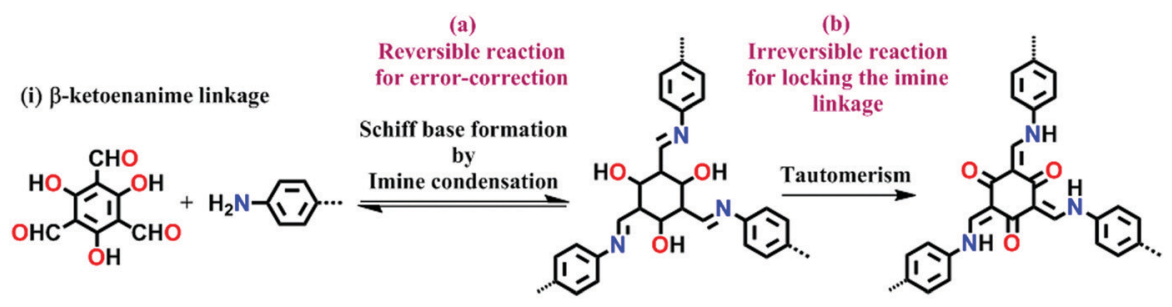

(ii) benzimidazole, benzobisoxazole and thiazole linkage

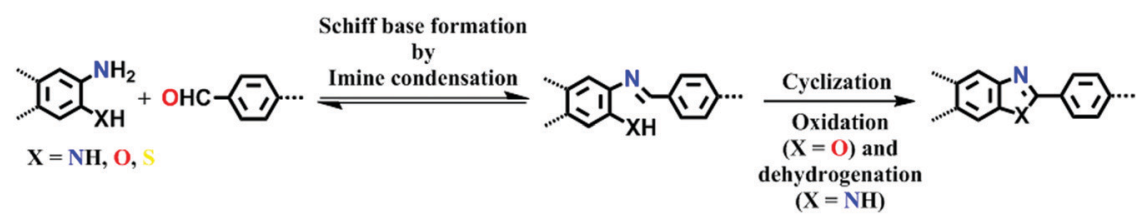

Fig. 4 Strategy for the synthesis of highly stable $\mathrm{N}$-containing linkages by (a) reversible and (b) irreversible reactions.
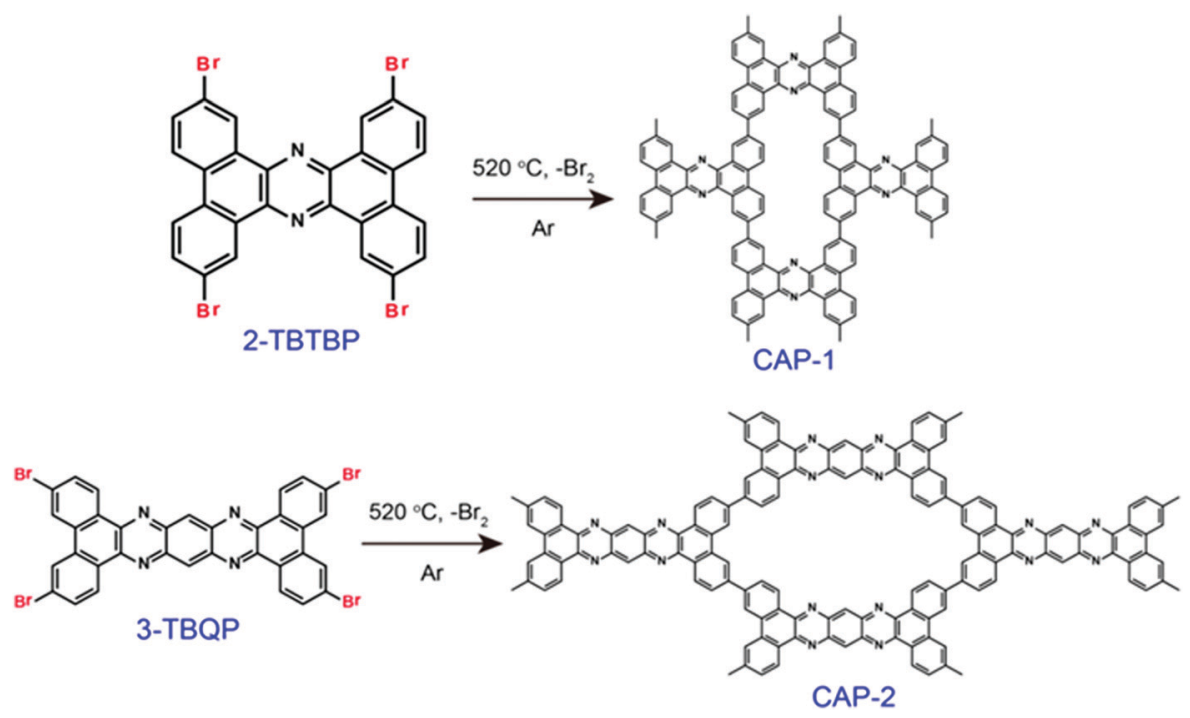

Fig. 5 Schematic representation of the formation of CAP-1 and CAP-2 by irreversible C-C coupling reactions. Reproduced with permission from ref. 72 , Copyright the American Chemical Society (2018).

the crystallinity of $2 \mathrm{D}$ sheets. ${ }^{76}$ Accordingly, the requirement of rigid, planar and highly symmetric monomers should be fulfilled. Moreover, factors such as the choice of solvent for the reaction, temperature, effect of modulator added, degree of freedom, rate of the addition of monomers and amount of by-product in the reaction largely determine the course of the reaction and product stability. ${ }^{77}$ The size, shape and type of pores constructed during this process can be predetermined by the topological design (vide supra). Once stable covalent organic sheets are formed, the supramolecular interactions come into play. The adhesion of 2D sheets one-over-the other influences the bulk properties, such as surface area, luminescence, and conductivity. For instance, strong $\pi-\pi$ interactions between stacked COF sheets containing a pyrene moiety cause the ACQ effect (vide infra).

Various reversible linkages have been proposed to gain an ordered 2D sheet (discussed in Section 3.1). On the contrary, the supramolecular interactions that stick the planar sheets together are somewhat less explored. ${ }^{78} \mathrm{~A}$ few examples are discussed below to give insight into the different types of supramolecular interactions, depending on the geometry and orientation of the monomers.

Constructed with strong van der Waals forces. Boronic esterlinked COFs were the first COFs to be synthesized and wellstudied to discover their core chemistry. These frameworks are stacked on each other with strong van der Waals forces between two robust 2D COF layers. A detailed computational study was carried out on HHTP-DPB COF, where planar and highly rigid monomers were utilized under solvothermal conditions. ${ }^{18}$ The results from molecular mechanics and density functional theory indicate slight offset stacking $(\sim 1.7-1.8 \AA)$ of the layers, which is not feasible to determine using the common characterization methods, but can be observed by SCXRD. This offset places the boron atoms of one layer in close proximity to the O-atoms of the adjacent layer, thereby building strong dipole-dipole 
interactions, which stick the $2 \mathrm{D}$ sheets together. The results are more accurate with density functional theory given that it precisely considers the electrostatic interactions for simulation.

Constructed with aromatic $\pi-\pi$ stacking. The planarity of the monomers after their assembly within a framework is vital to achieve a good magnitude of certain bulk properties. An excellent example of this was reported in 2016 by Banerjee and co-workers, yielding two imine-based COFs 2,3-DhaTta and 2,3-DhaTab, via the combination of 2,3-dihydroxyterephthaldehyde with triazine and phenyl-core triamine, respectively (Fig. 6). ${ }^{79}$ During the reversible imine bond formation, it was reported that planarity of the triazine-core with respect to the peripheral phenyl rings was achieved with angles of $\sim 174^{\circ}-179^{\circ}$, whereas in the case of the phenyl core, deviation from planarity with angles of $\sim 145^{\circ}-154^{\circ}$ was observed. Intralayer $\mathrm{H}$-bond formation between the imine $\mathrm{N}$ and diols caused additional planarity to the $2 \mathrm{D}$ sheet, although there was a tilt of $\sim 134^{\circ}$ between the triamine and terephthaldehyde units. Consequently, strong aromatic $\pi-\pi$ stacking was observed in 2,3-DhaTta, forming ribbon-like structures in comparison to 2,3-DhaTab with a hollow sphere. This was evident from the BET surface area calculations with a high surface area of $1700 \mathrm{~m}^{2} \mathrm{~g}^{-1}$ for the more planar and stacked 2,3-DhaTta, which also showed nine-times more intense PXRD reflections (100 to 001 plane) compared to the value of $413 \mathrm{~m}^{2} \mathrm{~g}^{-1}$ for 2,3-DhaTab.

Constructed with hydrogen bonding. Some cases have been recorded where the $2 \mathrm{D}$ COF sheets are stacked together by hydrogen bonding. This requires that the atoms with the ability to form hydrogen bonds be oriented in close proximity to fulfil the hydrogen-bonding conditions. As examples, six different COFs have been reported, namely TPOMe-Tab, TPOMe-PaNO TPOMe-Pa1, TPOMe-BD $\left(\mathrm{NO}_{2}\right)_{2}$, TPOMe-BPy and TPOMe-Azo, which were synthesized from trimethoxy-substituted benzenetricarboxaldehyde (TPOMe) with six different diamines. ${ }^{80}$ Except for the TPOMe- $\mathrm{BD}\left(\mathrm{NO}_{2}\right)_{2} \mathrm{COF}$, they all have OMe groups perpendicular to the planar COF sheets, which favourably form interlayer hydrogen bonds. In addition, the methoxy groups provide steric hindrance around them, which generates a hydrophobic environment for the framework as a whole. Hence, the COFs resist very harsh acidic (18 M conc. $\mathrm{H}_{2} \mathrm{SO}_{4}$ and $12 \mathrm{M}$ conc. $\mathrm{HCl}$ for $7 \mathrm{~d}$ ), basic ( $9 \mathrm{M} \mathrm{NaOH}$ for $1 \mathrm{~d}$ ) and moisture conditions. The utilization of flexible monomers for the construction of hydrogen-bonded 2D COFs is a much more laborious challenge than that with planar monomers. Thus, only the urea-functionalized COFs, COF-117 and COF-118, reported by Yaghi and co-workers in 2018 give a comparative study to emphasize this point. ${ }^{81}$ The variation in their surface areas, i.e., $114 \mathrm{~m}^{2} \mathrm{~g}^{-1}$ (COF-117) and $1524 \mathrm{~m}^{2} \mathrm{~g}^{-1}$ (COF-118), is due to the different conformations of the urea molecule. The disruption of the $\mathrm{C}-\mathrm{N} \cdots \mathrm{H}$ bond in COF-117 is attributed to the bond rotations in the pliable units, which were confirmed by FTIR spectroscopy before and after the introduction of acetonitrile solvent guest molecules.

As discussed in the preceding paragraphs, there are numerous examples of 2D COFs with supramolecular interactions of various

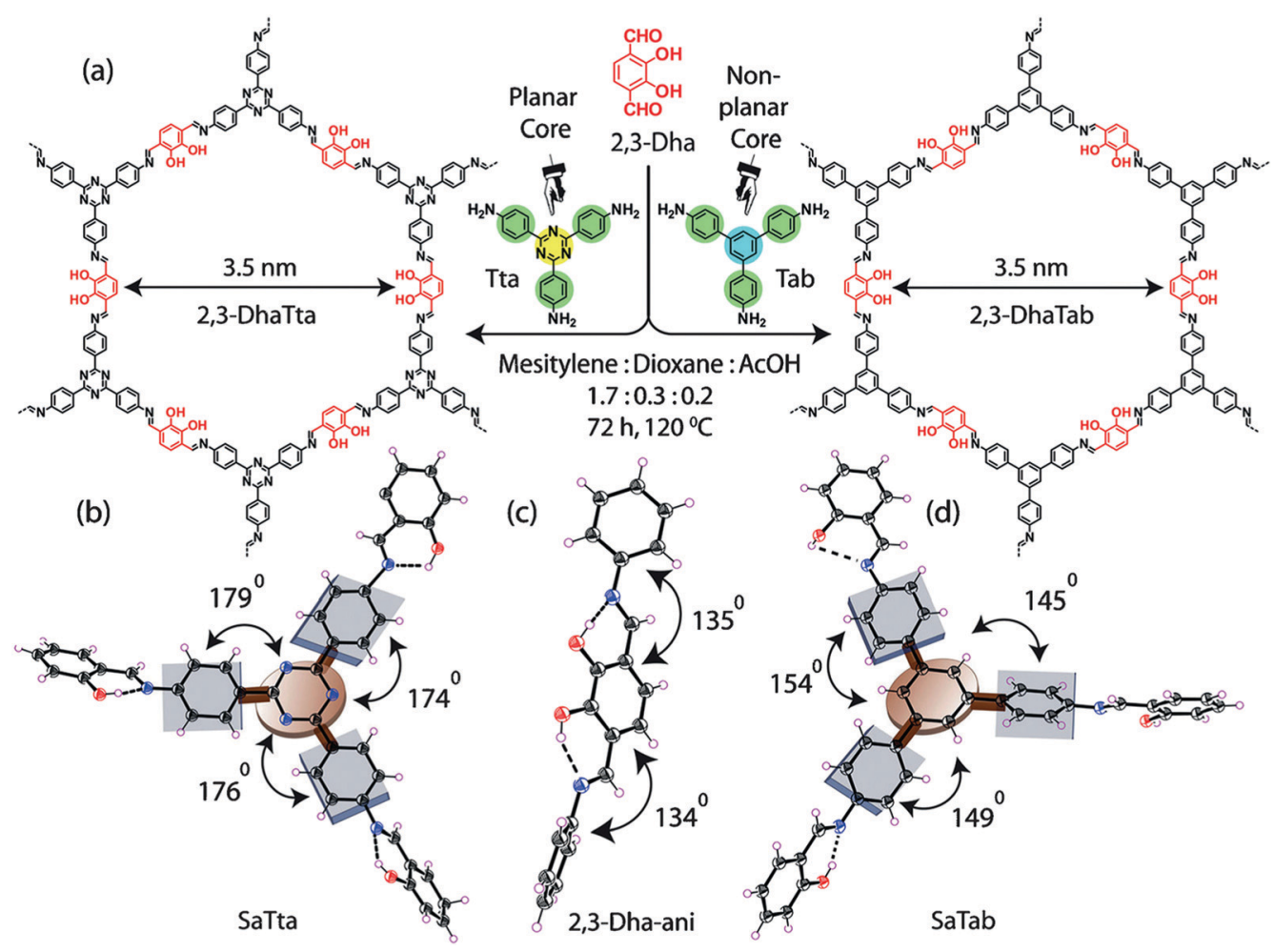

Fig. 6 (a) Strategy for the formation of COFs 2,3-DhaTta and 2,3-DhaTab. (b-d) Angle between the planes of triazene and phenyl core with phenyl substituents and that with a diol moiety. Reproduced with permission from ref. 79, Copyright John Wiley and Sons (2016). 
types that produce microcrystalline powders. Supramolecular interactions, which are weaker in comparison to covalent bonds, provide reversibility to the reaction, but still it is difficult to obtain single crystals of 2D COFs. A detailed analysis of this has been extensively done by various groups, elaborating various factors in controlling the crystallinity of 2D COFs (see Section 4.3).

3.2.2 3D COFs. The synthesis of 3D COFs involves the reaction of rigid, non-planar and symmetric organic monomers, forming strong covalent bonds with high bond energies $\left(>500 \mathrm{~kJ} \mathrm{~mol}^{-1}\right)$. The 3D framework is chiefly associated with covalent bonding, which is absolutely irreversible, and therefore requires input to yield crystalline products. However, despite the problems associated with the difficulty of error-correction and reticular chemistry complications, a few single crystals of 3D covalently-bonded organic networks have been successfully reported. The three different categories of $3 \mathrm{D}$ COFs that can be separately outlined based on the information available in the literature are presented below.

Polycrystalline. Most 3D COFs are polycrystalline solids prepared mainly via the solvothermal technique. To generate highly ordered 3D COFs, the reaction conditions must be monitored in a proper and regular manner. Various parameters such as choice of solvents and their optimized composition, reaction temperature and environment (vacuum, $\mathrm{N}_{2}$ or air), time required for completion of the reaction, and amount of catalyst added are the major factors that control the reversibility of the reaction, yielding a multi-crystalline material. As mentioned in Section 3.1, the various linkages developed thus far use dynamic covalent chemistry to achieve the desired crystallinity.

The very first polycrystalline 3D COF was reported to possess a boroxine and boronic-ester linkage, which was synthesized solvothermally in 2007 by Yaghi and co-workers. ${ }^{45}$ Subsequently, the formation of the first imine-linked 3D COF was reported in 2009 by the same group. ${ }^{82}$

Since then, many of these frameworks have been reported by solely controlling the reaction conditions. Several other greener techniques have also been used to increase the uniformity and maintain high dimensionality in COFs, including mechanochemical, ${ }^{83}$ microwave-assisted, ${ }^{84}$ ionothermal, ${ }^{85}$ devitrification ${ }^{86}$ and linker exchange methods ${ }^{87}$ (see Section 4.1 for details). Basically, devitrification is the process of obtaining a crystalline material from an amorphous material. CAF-1 and CAF-2 with longrange order were synthesized by heating the corresponding amorphous polyamide networks, namely PATnC and PATCnC, respectively, at $240-250{ }^{\circ} \mathrm{C}$ for 7 days in the presence of 15 meq water. ${ }^{86} \mathrm{In}$ addition, a 3D-to-3D COF transformation happened from COF-320 to COF-300 via a heterogeneous linker exchange method. ${ }^{87}$ Initially, the 3D COF-320 net was formed solvothermally from biphenyl-4,4' dicarbaldehyde (BPDA) and tetra-4-anilylmethane (TAM). Afterwards, COF-320 was subjected to the linker exchange process with terephthaldehyde (TA) under solvothermal conditions, yielding COF-300. An appropriate amount of acid catalyst was required to activate the TA linker for the exchange reaction, as shown in Fig. 7a.

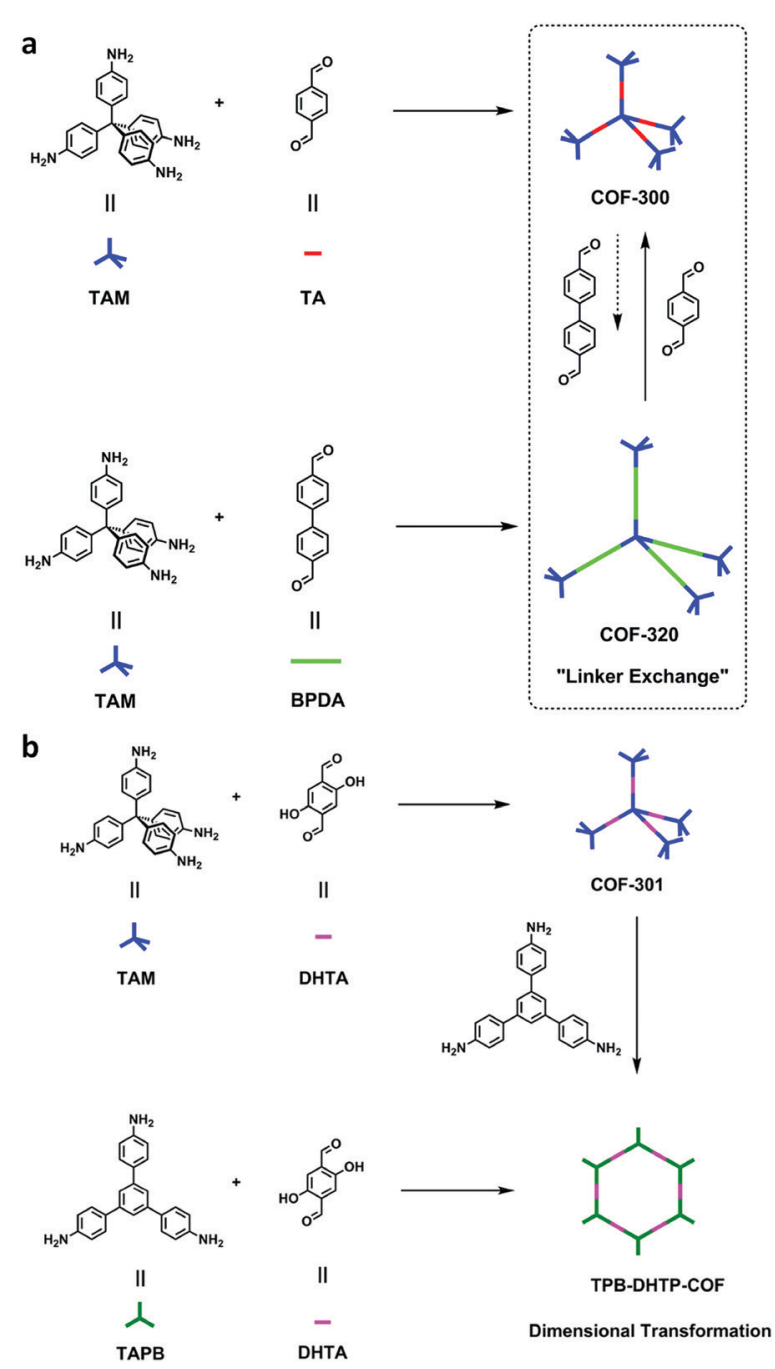

Fig. 7 (a) Synthesis of COF-300-to-320-X and COF-320-to-300- $X$. (b) Synthesis of COF-301-to-TPB-DHTP-COF-X $((X=(n 1 / n 2) \times 100$, where $n 1 / n 2$ is the molar ratio of the exchanging monomer to initial COF; $X=5,10$, and 15). Reproduced with permission from ref. 87 , Copyright the American Chemical Society (2019).

Single-crystalline. In recent years, mechanistic studies have focused on insight into improving the crystallinity of 2D COFs, while single crystals have only been obtained for 3D COFs. The first single crystal of a 3D COF was reported in 2013, namely, NPN-1, NPN-2 and NPN-3, which were synthesized via the oxidation of hydroxylamines, yielding tetrakis(4-nitrosophenyl)substituted methane, silane and adamantane, and subsequent polymerization yielded large and uniform crystals of approximately $0.5 \mathrm{~mm}$ in size (for NPN-3). ${ }^{88}$ In the same year, Zhang et al. proposed the crystal structure of the 3D imine-linked COF-320 (with size of $\sim 200 \mathrm{~nm}$ ) using the rotation electron diffraction technique (Section 5.4.2). The 9-fold interpenetrated net was woven solvothermally using tetra-(4-anilyl)methane and 4,4'biphenyldialdehyde with the dia topology. ${ }^{89}$ Fortunately, largesized single crystals $(\sim 60 \mu \mathrm{m})$ of 3D imine-based COFs were obtained, namely COF-300 (hydrated), COF-303, LZU-79, and LZU-111 with dia and lon nets (Section 2, vide supra), which 
were successfully diffracted using the SCXRD technique (Section 5.3.2). ${ }^{6}$ This propelled the expansion of single-crystal COF chemistry, emphasizing certain requirements.

Interpenetrated nets. Another remarkable feature of 3D frameworks is their interpenetrating capability to give a thermally strong and stable net. Almost all 3D COFs become tangled during their synthesis, and only a few examples are have been reported with non-interpenetration (cited in Section 2). The highest interpenetration in a 3D COF was recently reported with the chiral COF-16, which forms a 11-fold interpenetrated diamondoid-network. ${ }^{90}$ A more interesting interpenetration isomerism was witnessed by Ma et al. in 2018, where COF-300 was found to exist in two distinct isomers, dia-c7 and dia-c5, based on its degree of interpenetration. ${ }^{91}$ The factor of aging plays a role in their synthesis given that the formation of COF-300 dia-c5 requires a three-day solvothermal process, whereas COF-300 dia-c7 was synthesized in 9 days with a gradual increase in temperature from $25{ }^{\circ} \mathrm{C}$ to $50{ }^{\circ} \mathrm{C}$, and finally $120{ }^{\circ} \mathrm{C}$ for 3 days each.

3.2.3 Dimensionality exchange in COFs. As discussed above, 2D and 3D COFs are known to exist with a variety of linkages and network designs. Both have distinct synthetic routes and outstanding properties. A recent and unique report by Li et al. indicated that the dimensionality of COF-301 is reduced from 3D-to-2D TPB-DHTP COF by heterogeneous linker exchange (Fig. 7b). ${ }^{87}$ This is achieved by the replacement of a $C_{4}$-symmetric $T_{\mathrm{d}}$-tetraamine linker by $C_{3}$-symmetric triamine, where the dia net opens and changes to an hcb net. The noteworthy point here is that the use of the reversible imine bond formation reaction during the solvothermal synthesis of COF-301 makes ligand exchange possible. The imine bond hydrolyses in aqueous conditions, and therefore the second triamine ligand gets a chance to bind and reform the framework of lower dimensionality. Another report on switching the COF dimensionality from $2 \mathrm{D}$ to $3 \mathrm{D}$ was reported by Gao et al. ${ }^{92}$ The 2D-BPTA-COF was synthesized solvothermally using the planar building units -tetra( $p$-aminophenyl)-biphenyl (BPTA) and tetrakis-(4-formylphenyl) benzene
(TPBH) via $\left[C_{4}+C_{4}\right]$ imine condensation, yielding AB-stacked 2D sheets. Interestingly, by substituting the BPTA monomer with a methyl group, the two planar biphenyl rings (with an initial dihedral angle of $\sim 0^{\circ}$ ) suffer steric hinderance and become twisted, attaining a dihedral angle of $60^{\circ}$. Therefore, a highdimensional COF, namely 3D-BMTA-COF, was formed from the combination of tetra( $p$-aminophenyl)-bimesitylene (BMTA) and TPBH monomer. The new framework formed had the pts topology with 7-fold interpenetration.

\section{Synthesis}

The synthesis of robust porous organic polymers via covalent bonds with in-built crystallinity is challenging. The comprehensive discussion in the previous sections elaborates the importance of the reversible dynamic covalent chemistry for the synthesis of COFs. COF chemistry requires thermodynamically controlled organic reactions, which help in correcting and reforming the wrong bond and proofreading each step. ${ }^{93}$ Several synthetic methods have been developed to incorporate crystallinity and stability in COFs, making them potent materials for sensing applications. Recently, crystalline COF powders and films have been extensively utilized as sensory materials, which have been synthesized using various methods, as mentioned in detail below.

\subsection{General synthetic methods}

In this section, we discuss the various methods utilized for the synthesis of COFs and modifications for their applications. All the reported synthetic methods are different in terms of maintaining the reaction conditions, choice and amount of solvent, reaction temperature, time consumed, requirement of catalysts, and the source of energy, which helps in the formation of a particular framework in each case. A detailed comparison is shown in Table 2.

4.1.1 Solvothermal method. This is the most prevalent method for yielding crystalline bulk COF powders. In this method, a mixture of monomer units in an appropriate solvent

Table 2 Comparison of the different methods reported for the synthesis of COFs

\begin{tabular}{|c|c|c|c|c|c|}
\hline Synthetic method & $\begin{array}{l}\text { Energy } \\
\text { source }\end{array}$ & Reaction conditions & Solvent environment & Reaction time & Product formation \\
\hline Solvothermal & $\begin{array}{l}\text { Thermal } \\
\text { energy }\end{array}$ & $\begin{array}{l}\text { - High temperature and } \\
\text { autogenous pressure } \\
\text { - High vacuum } \\
\text { conditions }\end{array}$ & $\begin{array}{l}\text { - High boiling solvents } \\
\text { - Non-polar solvents reduce } \\
\text { the reaction homogeneity, } \\
\text { which further increases time } \\
\text { to attain thermodynamically- } \\
\text { controlled product } \\
\text { - Freeze-pump-thaw process } \\
\text { for proper degassing of } \\
\text { solvents }\end{array}$ & - 2 to 9 days & $\begin{array}{l}\text { - Gram-scale (comparatively } \\
\text { high yields for } 2 \text { D COFs are seen) } \\
\text { - Interpenetration reduces the } \\
\text { \%yield } \\
\text { - Depends upon the solubility } \\
\text { and reactivity of the starting } \\
\text { materials }\end{array}$ \\
\hline Mechanochemical & $\begin{array}{l}\text { Mechanical } \\
\text { energy }\end{array}$ & $\begin{array}{l}\text { - Ambient conditions } \\
\text { - Constant grinding }\end{array}$ & $\begin{array}{l}\text { - No or minimum solvent is } \\
\text { required }\end{array}$ & $\begin{array}{l}\text { - Several minutes to a } \\
\text { few hours }\end{array}$ & $\begin{array}{l}\text { - Good yields obtained but } \\
\text { with lower surface areas }\end{array}$ \\
\hline $\begin{array}{l}\text { Microwave- } \\
\text { assisted }\end{array}$ & $\begin{array}{l}\text { Microwave } \\
\text { radiations }\end{array}$ & $\begin{array}{l}\text { - Moderate temperature } \\
\text { and autogenous pressure }\end{array}$ & $\begin{array}{l}\text { - No harsh solvent conditions } \\
\text { are required }\end{array}$ & $\begin{array}{l}\text { - Faster compared to } \\
\text { other methods }\end{array}$ & $\begin{array}{l}\text { - Higher yields but with } \\
\text { reduced surface areas }\end{array}$ \\
\hline lonothermal & $\begin{array}{l}\text { Thermal } \\
\text { energy }\end{array}$ & $\begin{array}{l}\text { - Extremely high tem- } \\
\text { perature conditions }\end{array}$ & $\begin{array}{l}\text { - Ionic solids in molten form } \\
\text { act as solvents and catalyse the } \\
\text { reaction }\end{array}$ & $\begin{array}{l}\text { - Prolonged reaction } \\
\text { time required to reduce } \\
\text { side-product formation }\end{array}$ & $\begin{array}{l}\text { - Good yields but limited } \\
\text { crystallinity of frameworks }\end{array}$ \\
\hline $\begin{array}{l}\text { Room temperature } \\
\text { synthesis }\end{array}$ & $\begin{array}{l}\text { No external } \\
\text { energy }\end{array}$ & $\begin{array}{l}\text { - Ambient conditions } \\
\text { - Constant stirring }\end{array}$ & $\begin{array}{l}\text { - Solvents are chosen } \\
\text { according to solubility of the } \\
\text { monomers }\end{array}$ & $\begin{array}{l}\text { - Several minutes to a } \\
\text { few hours }\end{array}$ & $\begin{array}{l}\text { - Reduction of yields of well- } \\
\text { defined frameworks due to } \\
\text { solubility }\end{array}$ \\
\hline
\end{tabular}


combination is kept in an airtight container and sonicated and degassed via the freeze-pump-thaw method. The reaction mixture is then heated at high temperatures of $\sim 70-120{ }^{\circ} \mathrm{C}$ for $2-9$ days under vacuum. This helps in maintaining an appropriate environment for the reaction, achieving high crystallinity and stability in the framework. A large number of crystalline, porous and highly stable COF powders has been synthesised using this method. ${ }^{4,57,68,82}$ The reaction parameters such as temperature and pressure direct the reaction towards a thermodynamic output, which ensures long-range order in the framework. Moreover, the solubility of the reactants can be controlled by using a combination of solvents. The use of a modulator also plays a crucial role in introducing reversibility in the reaction.

4.1.2 Mechanochemical method. This method for the synthesis of COF powders is different from the other methods due to its greener approach. In this method, the organic monomers are placed in mortar and finely ground with a pestle for several minutes to yield highly ordered COFs. The energy required for the formation of persistent organic frameworks is provided by mechanical energy. For instance, the TpPa-COF series was constructed via solvent-free mechanical grinding under mild temperature conditions. ${ }^{83}$ To increase the reaction rate during synthesis, liquid-assisted grinding (LAG) is carried out, which involves the addition of a trace amount of catalytic solvent to the reaction mixture. ${ }^{94}$ This maintains the homogeneity in the reaction and mixes the reactants well to ensure monomer availability, which helps in attaining high periodicity. Other COFs with various linkages synthesized using the LAG approach are discussed in detail below.

4.1.3 Microwave-assisted method. Among the various synthetic methods, microwave-assisted COF synthesis takes the least time to complete the reaction. It accelerates the rate of the reaction to support the rapid synthesis of crystalline materials with high BET surface areas. In this method, the reactants are dissolved or dispersed in a suitable solvent mixture via sonication, and then sealed in a glass Pyrex tube, which has either a vacuum or $\mathrm{N}_{2}$ environment. The sealed tube is subjected to microwave irradiation together with heating for a short period (20-60 min). The boronic ester-linked COFs MW-COF-5 and MW-COF-102 were synthesized at moderately high temperature in $20 \mathrm{~min},{ }^{95}$ while a dioxin-linked TH-COF (also called COF-318) took $30 \mathrm{~min}$ for completion of the reaction using this approach. ${ }^{96}$

4.1.4 Ionothermal method. In this method a mixture of monomers and $\mathrm{ZnCl}_{2}$, sealed in a Pyrex tube, is evacuated and heated to high temperature $\left(400{ }^{\circ} \mathrm{C}\right)$ for a prolonged time. At the elevated temperature, the molten salt acts as a catalyst, the excess of which is washed away with dilute $\mathrm{HCl}$ solution. COF powders with moderate surface areas $\left(\sim 500-900 \mathrm{~m}^{2} \mathrm{~g}^{-1}\right)$ and pore volume are fabricated using this method. The first crystalline CTFs, namely CTF-1 and CTF-2, were constructed using this method. ${ }^{20}$ The use of ionic liquids as a solvent for the synthesis of 3D-ILCOFs was proven to be a green synthetic approach. ${ }^{85}$ However, the ionothermal process has some limitations, for example, (a) the monomers must have the ability to withstand harsh reaction conditions and (b) the removal of the spent $\mathrm{ZnCl}_{2}$ is difficult, and thus its residue causes contamination of up to $5 \mathrm{wt} \%$.
4.1.5 Room-temperature synthesis. Bulk powder synthesis of highly crystalline and stable COFs has also been practiced under ambient conditions. The two monomers were mixed with an appropriate solvent combination and a suitable catalyst, and then stirred at room temperature for several hours. This method is simple and easy to operate. Thus, many porous organic frameworks with improved thermal stability and high surface areas have been synthesized using this strategy, for example RT-COF- $1{ }^{97}$ and NP@a-1. ${ }^{98} \mathrm{COF}_{25}$ was constructed via acidcatalysed room temperature synthesis, yielding a crystalline bulk powder with a high BET surface area of $1523 \mathrm{~m}^{2} \mathrm{~g}^{-1} \cdot{ }^{99}$

4.1.6 Interfacial method. COF thin films and nanosheets have been successfully produced using the bottom-up interfacial approach. In this strategy, the films are synthesized at narrow interfaces of liquid/liquid, ${ }^{100}$ solid/vapour ${ }^{101}$ or liquid/ air ${ }^{102}$ phases. In the liquid/liquid interfacial synthesis method, monomer $\mathbf{A}$ is dissolved in a suitable high density solvent $\mathbf{1}$ and placed as the bottom layer in the ampule. Then, a spacer layer (also called the buffer layer in MOF chemistry ${ }^{103}$ ) is placed on top of it, which can either be solvent 2 of a comparatively lower density or a mixture of two solvents $(\mathbf{1}: 2)$. The third layer contains monomer $\mathbf{B}$ immersed in solvent $\mathbf{2}$. The two solvents taken must be miscible at a slow rate to form smooth and periodic thin COF films.

Alternatively, the fabrication of solid/vapour interfacial thin films is carried out over an HOPG surface. In the typical synthetic procedure, a drop of monomer A solution is impinged over the substrate, and then introduced in a Teflon-sealed autoclave, which contains monomer B with $\mathrm{CuSO}_{4} \cdot 5 \mathrm{H}_{2} \mathrm{O}$. It should be noted that both monomers are not in direct contact. The whole system is sealed and subjected to high temperature for a few hours. Precursor $\mathbf{B}$ evaporates at high temperature and comes in contact with monomer A, resulting in the formation of covalent bonds at the solid/vapour interface. The rate of the vapour-phase monomer dosage decides the growth of the COF.

4.1.7 Exfoliation method. The delamination of bulk materials is another way to fabricate discrete thin films, which is a top-down strategy. In this method, the 2D COF sheets stacked by $\pi-\pi$ interactions are pushed away using different energy sources, including solvent-aided delamination, mechanical grinding, and chemical and self-exfoliation. Solvent-directed delamination is the most common approach, which introduces low boiling solvent molecules into the 2D powdered framework and the suspension is sonicated for $\sim 15-30 \mathrm{~min}$. On evaporation of the solvent, thin nanofilms with a thickness of $0.5-10 \mathrm{~nm}$ are obtained. COF- $1,{ }^{104}$ COF- $8,{ }^{105}$ COF- $43^{106}$ and TPA-COF ${ }^{107}$ were fabricated using the solvent-assisted exfoliation approach. Solvent-free mechanical grinding of bulk COF powders has proven to be a convenient method to yield thin films with a thickness of $\sim 3-10 \mathrm{~nm}$. TpPa and TpBD COFs were synthesized by grinding the bulk material at room temperature for $0.5 \mathrm{~h},{ }^{108}$ whereas DAAQECOF was milled with steel balls in two shifts for $30 \mathrm{~min} .{ }^{109}$

In some cases, it has been observed that the COF nanofilms lose their stability in the dispersion medium after they are peeled from the bulk powder. Therefore, the chemical exfoliation approach is carried out, which provides dispersion stability to 
the nanosheets. The nanosize of the thin films primarily varies with the concentration of the powder suspension. At a higher concentration, more sheets will be in proximity to form thick films. An example of this is the DaTp COF, where $N$-hexylmaleimide was used at high temperature to conduct delamination with great stability. ${ }^{110} \mathrm{~N}$-Hexylmaleimide undergoes Diels-Alder reaction with the anthracene units in Da and increases the hydrophobic alkylalkyl interactions among the 2D layers. The process was well monitored by FT-IR and PXRD analysis. Post-synthetic modification by chemical delamination was also performed during the thin film synthesis of the TpASH COF, which was further exploited for drug delivery appications. ${ }^{111}$

A few cases have been reported where self-exfoliation of the framework occurs, leading to the formation of thin nanofilms. In this case, the choice of the building units, which leads to delayering via internal force, is important. No external force is required for carrying out this process. Few-layered IISERPCON1 nanofilms with a thickness of $\sim 2-6 \mathrm{~nm}$ and aspect-ratio of $<0.1$ were fabricated using the above-mentioned technique. ${ }^{112}$ Moreover, a few ionic covalent organic nanosheets (iCONs) were self-exfoliated due to the interlayer repulsions caused by the positively charged guanidinium monomeric unit, including TpTG $x$ $\left(x=\mathrm{Cl}^{-}, \mathrm{Br}^{-}\right.$and $\left.\mathrm{I}^{-}\right)$iCONs ${ }^{113}$ possessing 3-6 layers with a size of 2-5 nm and DATG $_{\mathrm{Cl}} \mathrm{iCONs}^{114}$ possessing 13-18 layers with a size of 5-7 nm. The iCONs formed showed chemical and water stability and retained their ionic properties after self-exfoliation.

In addition to these methods, the continuous flow method ${ }^{115,116}$ and hydrothermal method ${ }^{117}$ were employed to produce direct and free-standing COF nanosheets via the bottom-up approach. Nanofilms of COF-5, which is the first COF reported to be formed by boronic ester linkages, with a thickness of $\sim 70-200 \mathrm{~nm}$ have been successfully formed.

\subsection{Reaction conditions for diverse linkages}

In this section, we selected examples from Table 3 for all the linkages mentioned in Fig. 3 to discuss the reaction conditions used for their synthesis. We apologise to researchers for not being able to include their examples given that it is very difficult to present all the examples available in the literature in this review. The heart of COF chemistry is the choice of the monomers, except in few cases, where it involves only one component such as boroxine and triazine-based COFs and the complementary monomers in the two-component system form the linkages. For nitrogen-rich COFs, there are four types of monomers, namely nitriles, amines, aldehydes and hydrazones. Furthermore, there are eleven nitrile-based, forty-eight aminebased, thirteen aldehyde-based and fourteen hydrazine-based monomers used thus far. Interestingly, the distribution of amines (twenty-five diamines, nine triamines, two $C_{2}$-symmetric tetramines, nine $C_{4}$-symmetric tetramines, one $C_{2}$-symmetric hexamine and three $C_{6}$-symmetric hexamines) is less than even aldehydes (thirteen dialdehydes, fifteen trialdehydes, eleven tetra-aldehydes and one hexa-aldehyde).

B-O linkage. The synthesis of covalent organic frameworks linked with small and light atoms ( $\mathrm{B}$ and $\mathrm{O}$ ) was first reported in 2005 by Yaghi and co-workers. ${ }^{4}$ COF-1 and COF-5 were constructed via the one-pot solvothermal technique, directing the formation of a six-membered planar boroxine ring and a fivemembered boronic-ester ring, respectively, by polycondensation reaction. The formation of the COF-1 crystalline bulk powder involves the co-condensation of 1,4-benzenediboronic acid (BDBA) at $120{ }^{\circ} \mathrm{C}$ for $72 \mathrm{~h}$ in a mesitylene/dioxane mixture in a sealed tube (Fig. 8). Similar reaction conditions were implemented for the bulk synthesis of COF-5 using 2,3,6,7,10,11-hexahydroxytriphenylene (HHTP) and phenyl boronic acid (PBA), involving the interaction between boronic acids and diols. Numerous microporous COFs with alkyl (i.e., methyl, ethyl or propyl) functionality were also synthesized through the reaction of dialkyl-substituted derivatives of 1,2,4,5-tetrahydroxybenzene ( $\left.\mathrm{R}_{2} \mathrm{THB}\right)$ with benzene-1,3,5-triboronic acid (BTBA). A series of COF networks with various pore diameters named COF-18 ( $\mathrm{R}=-\mathrm{H}), \mathrm{COF}-16 \AA$ ( $\mathrm{R}=-$ methyl), and COF-14 $\AA$ ( $\mathrm{R}=-$ ethyl) was obtained in $95-99 \%$ yield, while COF-11 $\AA$ ( $\mathrm{R}=-$ propyl) was formed in $65 \%$ yield. ${ }^{59}$

The faster synthesis of the COF-5 powder was conducted with the microwave-assisted technique, yielding a crystalline product in $20 \mathrm{~min}$ at $100{ }^{\circ} \mathrm{C}$ and $200 \mathrm{~W}$ power. ${ }^{95}$ The resulting framework possessed a higher BET surface area $\left(2019 \mathrm{~m}^{2} \mathrm{~g}^{-1}\right)$ in comparison to that obtained via the usual solvothermal method $\left(1590 \mathrm{~m}^{2} \mathrm{~g}^{-1}\right)$.

C-N linkage (triazine). Covalent triazine frameworks (CTFs) are analogs to zeolites, metal organic frameworks (MOFs), and covalent boron oxide-based frameworks (COFs) in terms of performance due to their high surface area and porosities. They are obtained via trimerization reaction under ionothermal conditions in the presence of molten $\mathrm{ZnCl}_{2}$ at high temperature (400-600 ${ }^{\circ} \mathrm{C}$ ) for $40 \mathrm{~h}$. It is noteworthy that the nitrile-substituted monomers are highly soluble in molten $\mathrm{ZnCl}_{2}$ salt, which eventually behaves as an efficient catalyst for the trimerization reaction. The reaction is adequately reversible at elevated temperatures, yielding thermally stable CTFs (above $400{ }^{\circ} \mathrm{C}$ ) with aromatic and heterocyclic-based nitrile monomers. CTF-1 was synthesized via the trimerization of dicyanobenzene (DCB) monomer in molten $\mathrm{ZnCl}_{2}$ with high crystallinity and a porous framework. ${ }^{20}$ Extended $\pi$-conjugation in the layered CTF-2 was obtained in similar manner via the ionothermal condensation of the highly rigid and chemically stable 2,6-naphthalenedinitrile (NDN) monomer. ${ }^{118}$ Zhang et al. reduced the reaction time from $40 \mathrm{~h}$ to $1 \mathrm{~h}$ by using the microwave-assisted polymerization approach. CTF-type polymers, namely P1-P6 and P1M-P6M, were synthesized by employing trifluoromethanesulfonic acid (TFMS) catalyst with six different nitrile-substituted aromatic monomers at room temperature and microwave-assisted conditions, respectively. ${ }^{119}$ The lowtemperature TFMS-catalyzed condensation has a significant advantage in that it avoids the undesirable decomposition due to carbon-hydrogen bond cleavage at high reaction temperature. Moreover, among the samples, polymers P6 and P6M exhibited the highest surface areas (1152 and $947 \mathrm{~m}^{2} \mathrm{~g}^{-1}$, respectively) due to their longest $T_{\mathrm{d}}$-symmetric tetra-nitrile-substituted monomer. Another triazine-linked framework, namely CTF-0, was prepared under similar ionothermal conditions using 1,3,5-tricyanobenzene (TCB). ${ }^{19}$ CTF-0 possesses a higher nitrogen content 


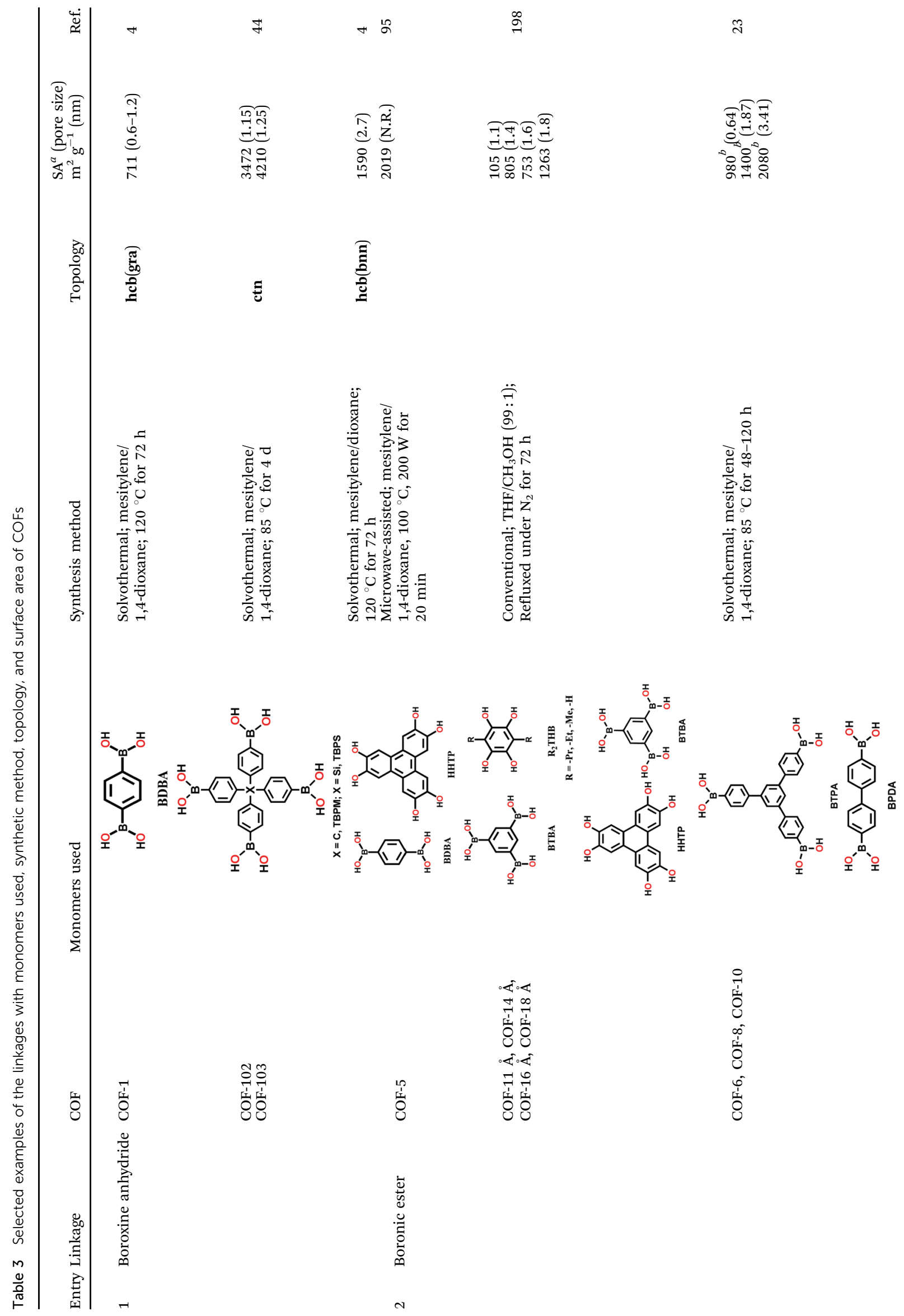




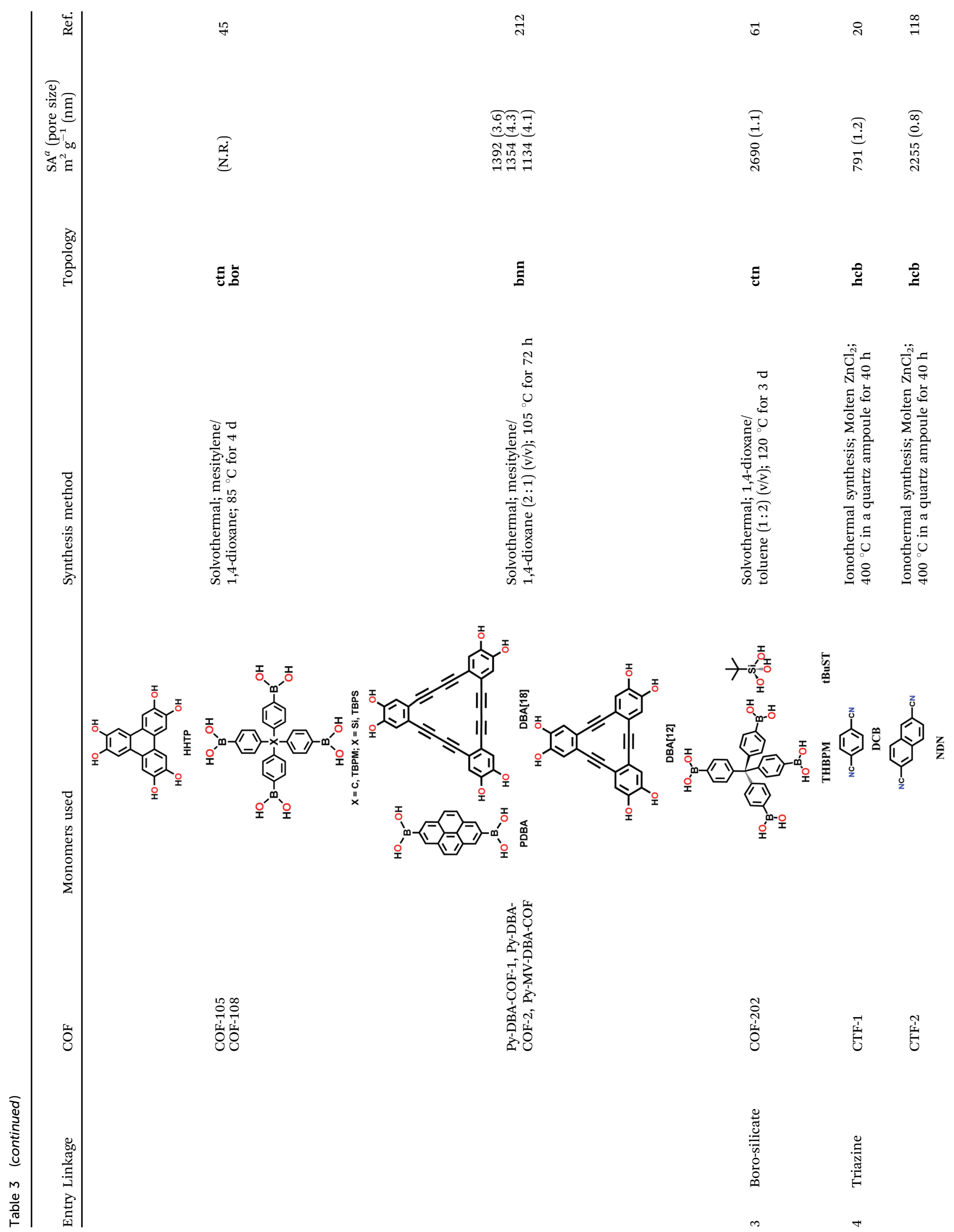




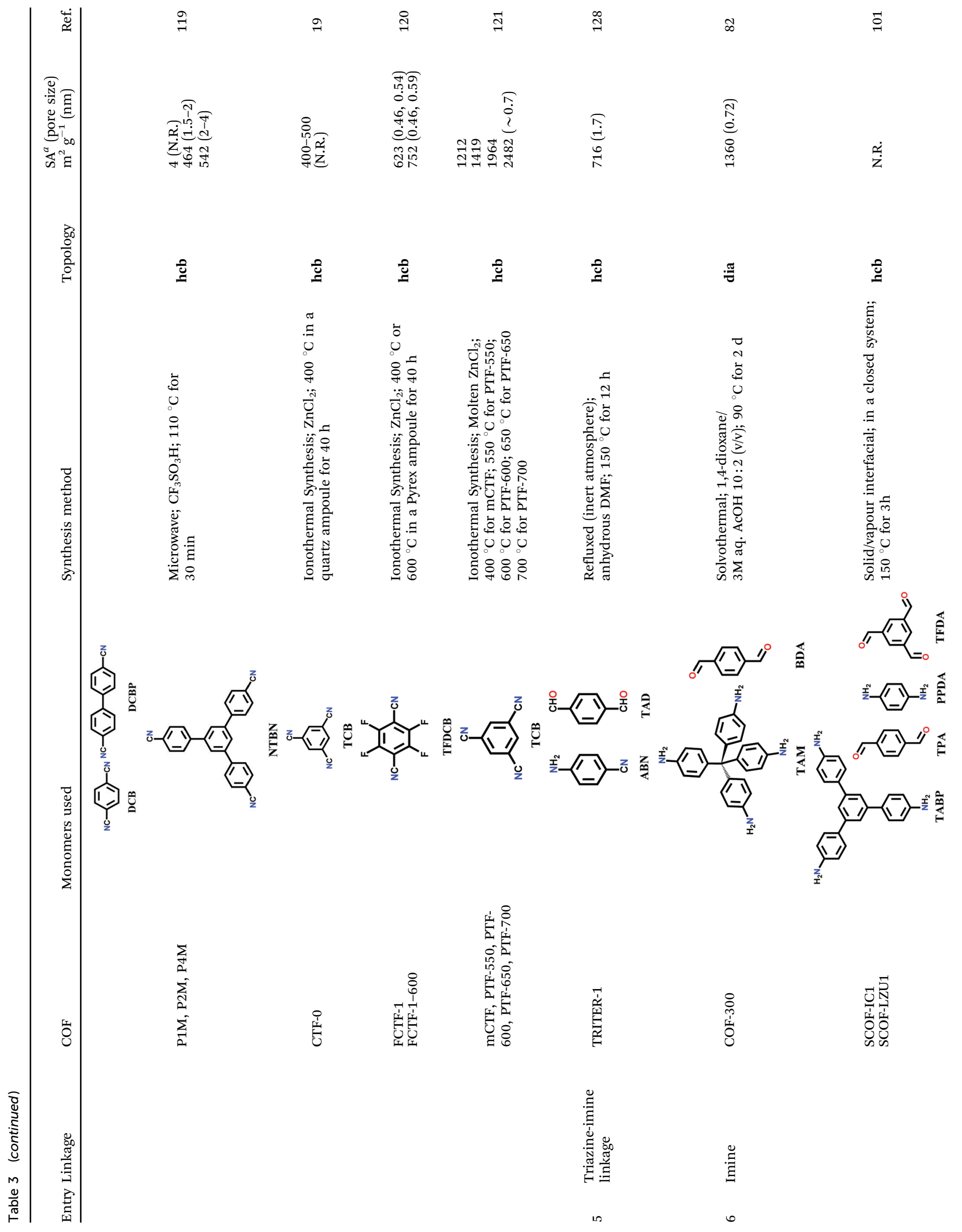


$\bar{s}$

$\stackrel{\Xi}{\underline{\Xi}}$

¿

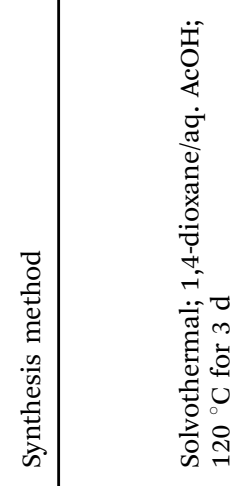

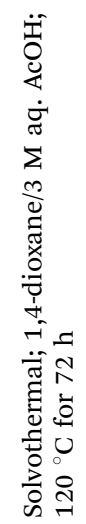

(n)

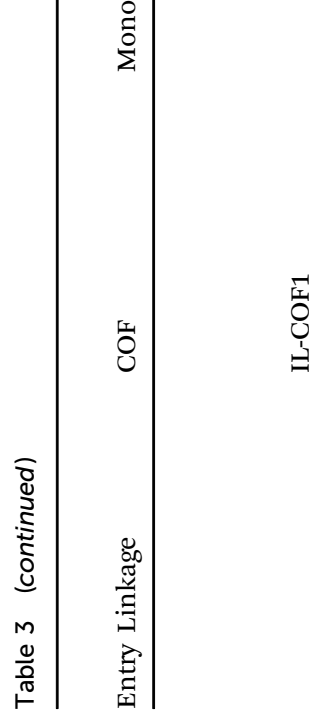

空 

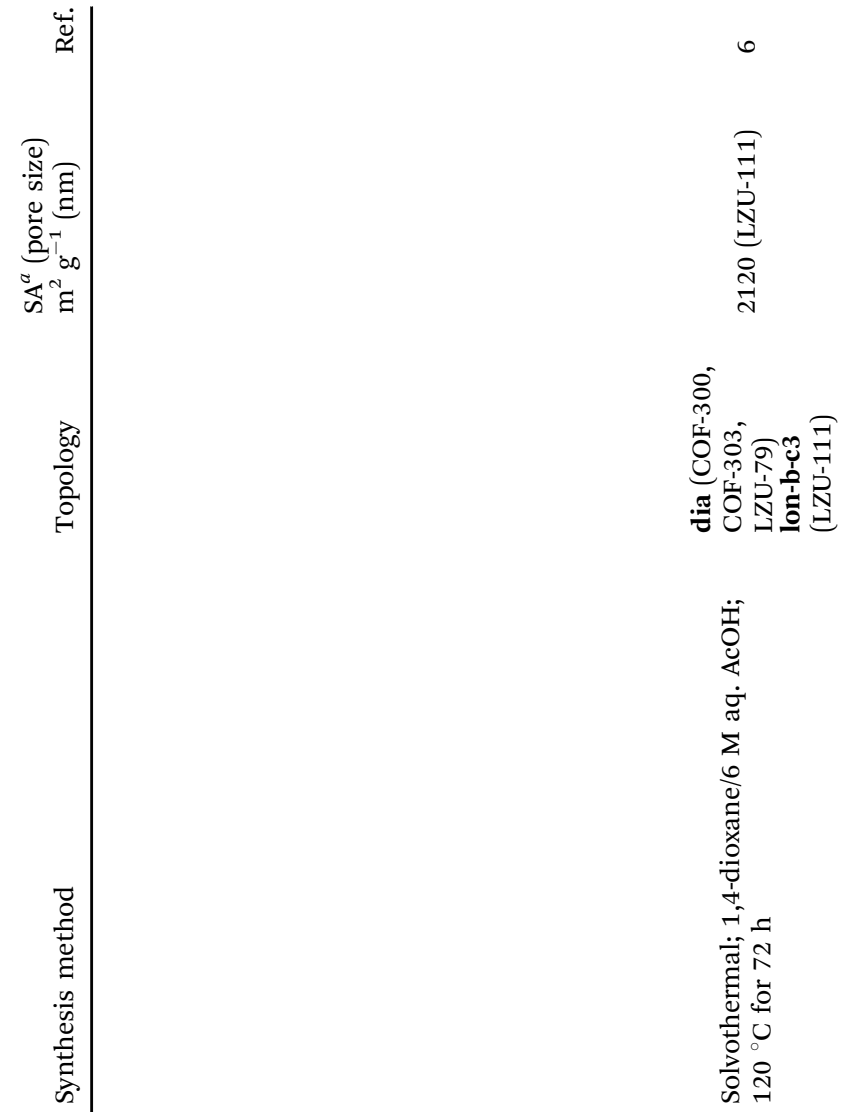

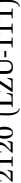

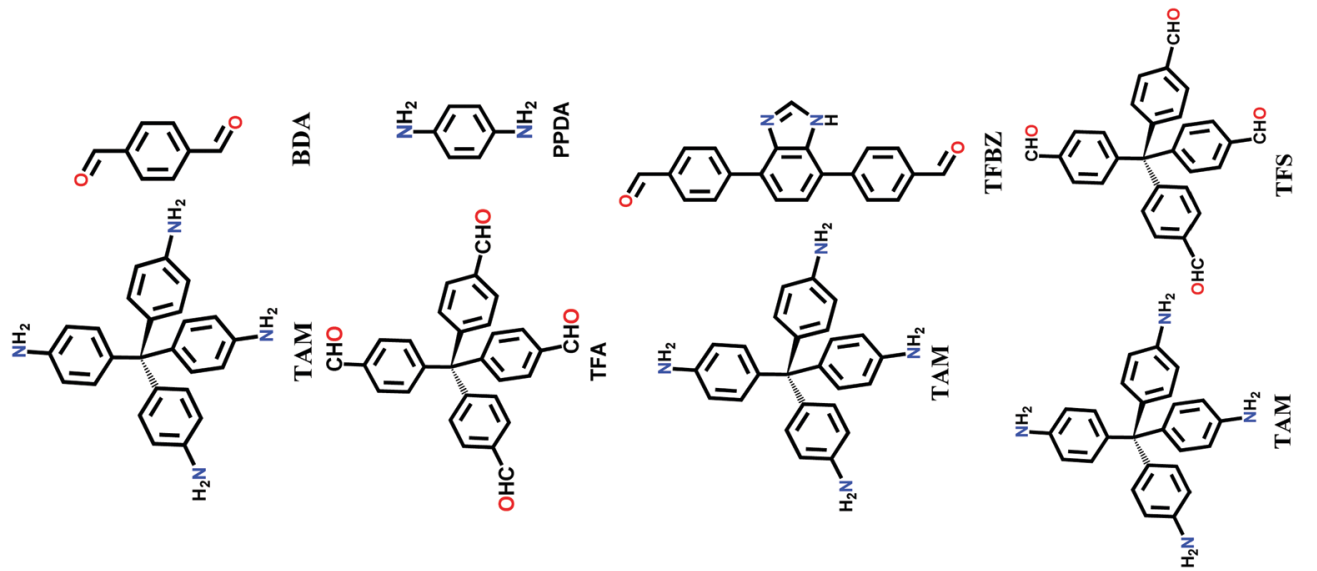




\section{Materials Advances}

View Article Online

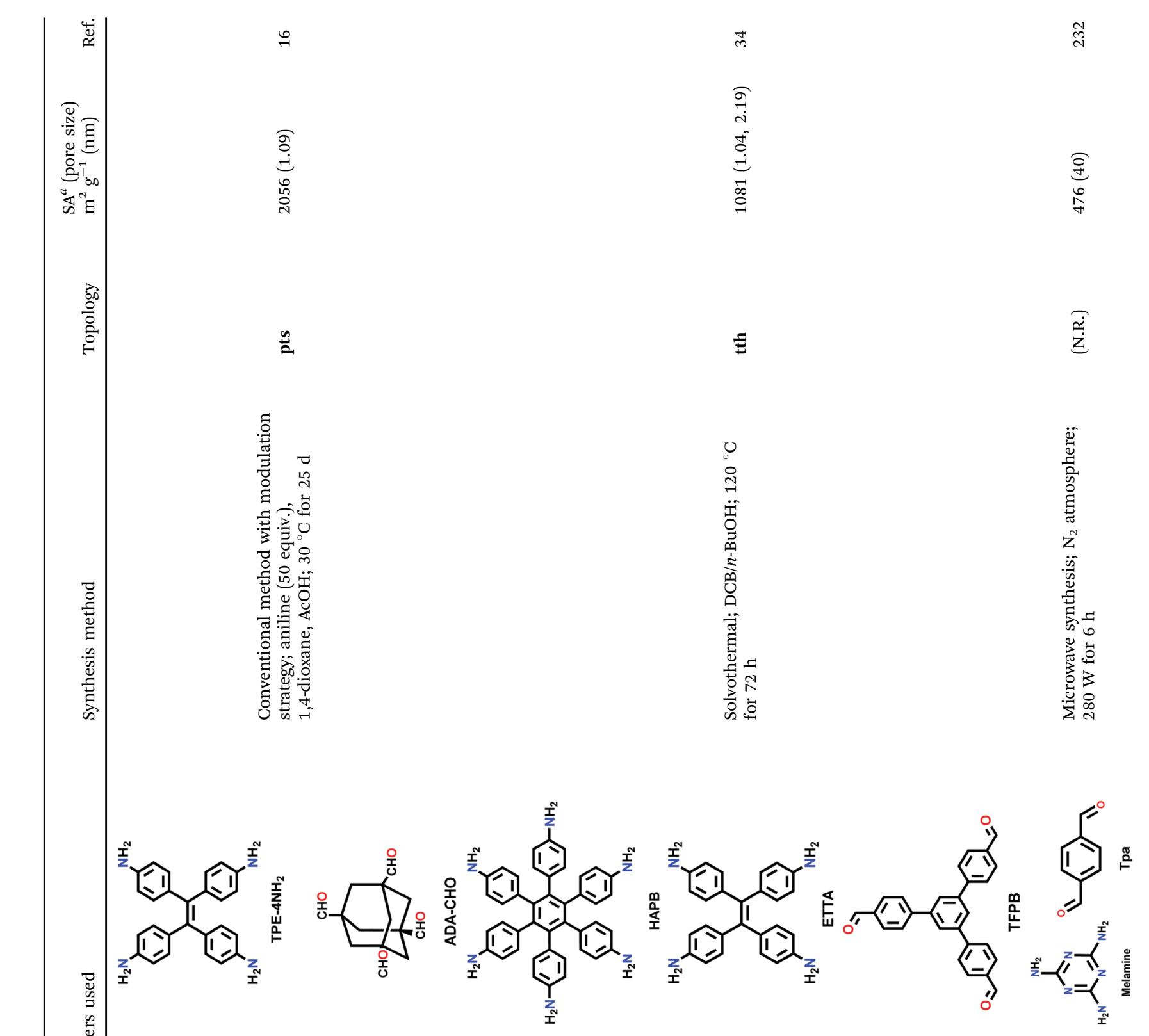

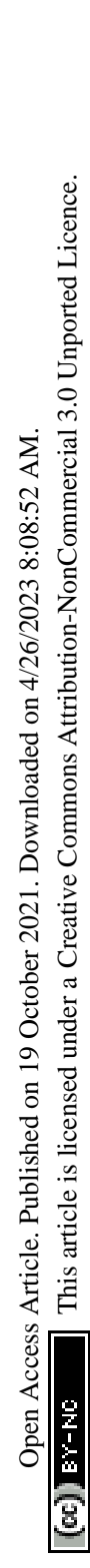

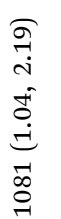

$\frac{4}{0}$

$\stackrel{\dot{2}}{\dot{2}}$ 

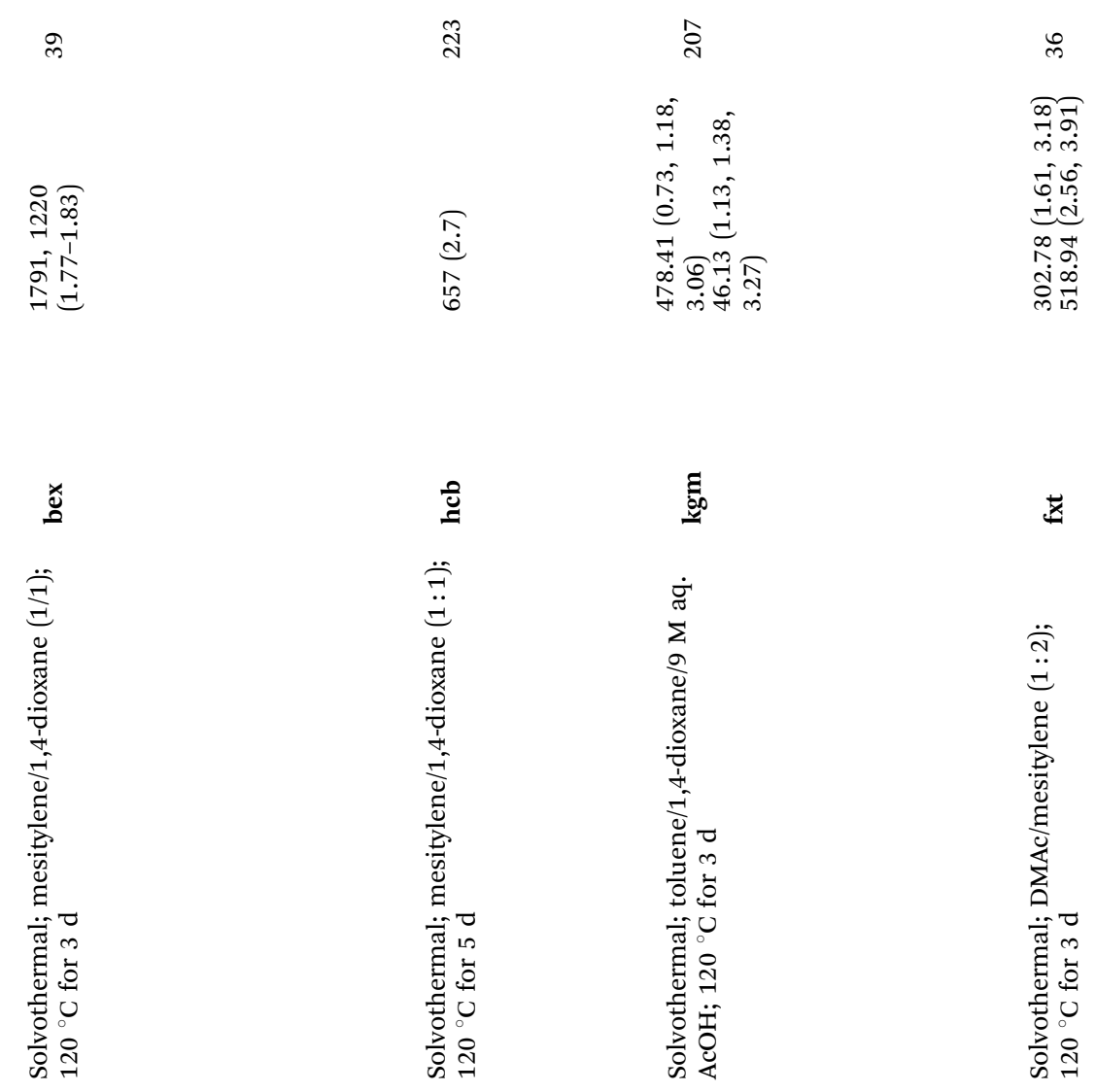

$\forall$
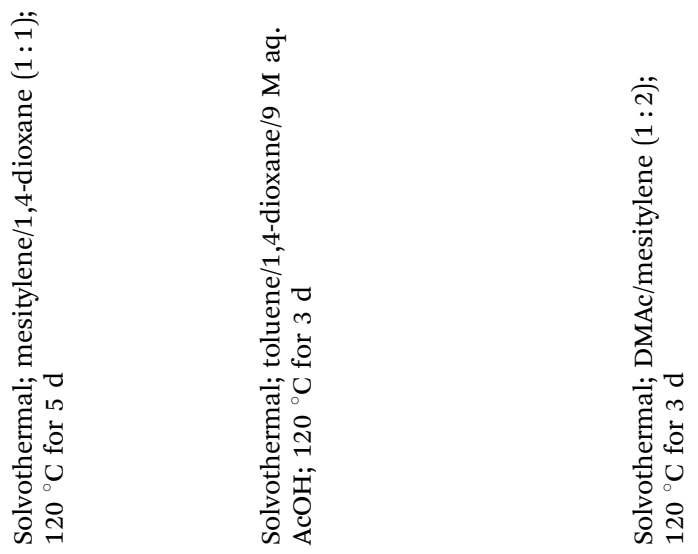

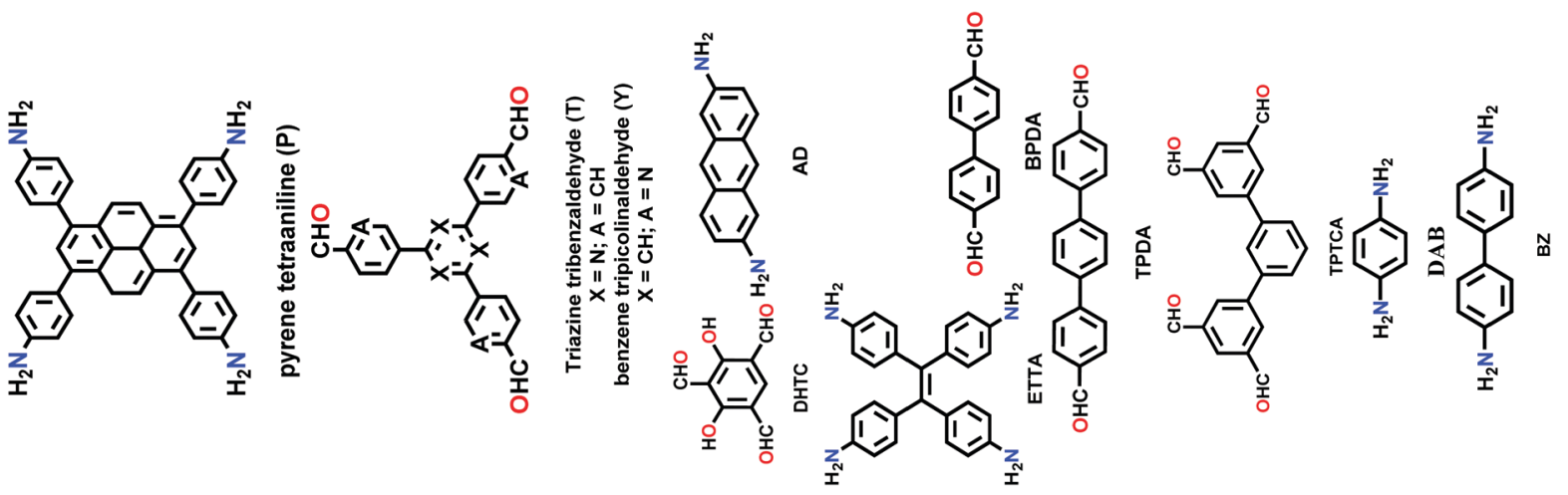

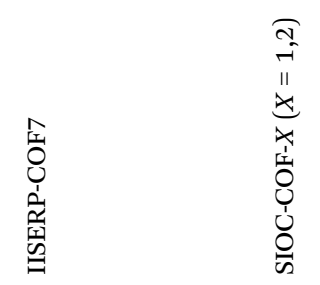

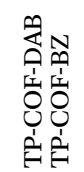


F

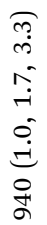

로

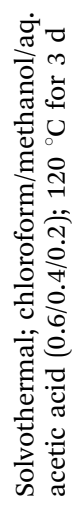

แn

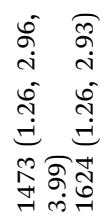

के

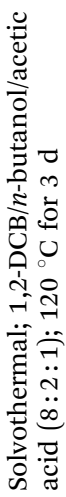

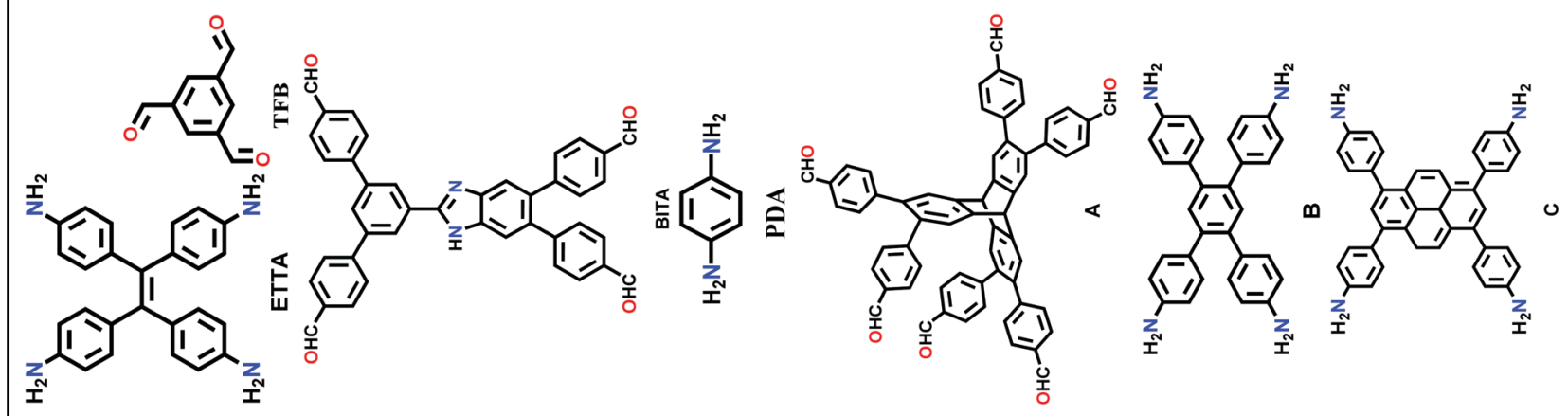




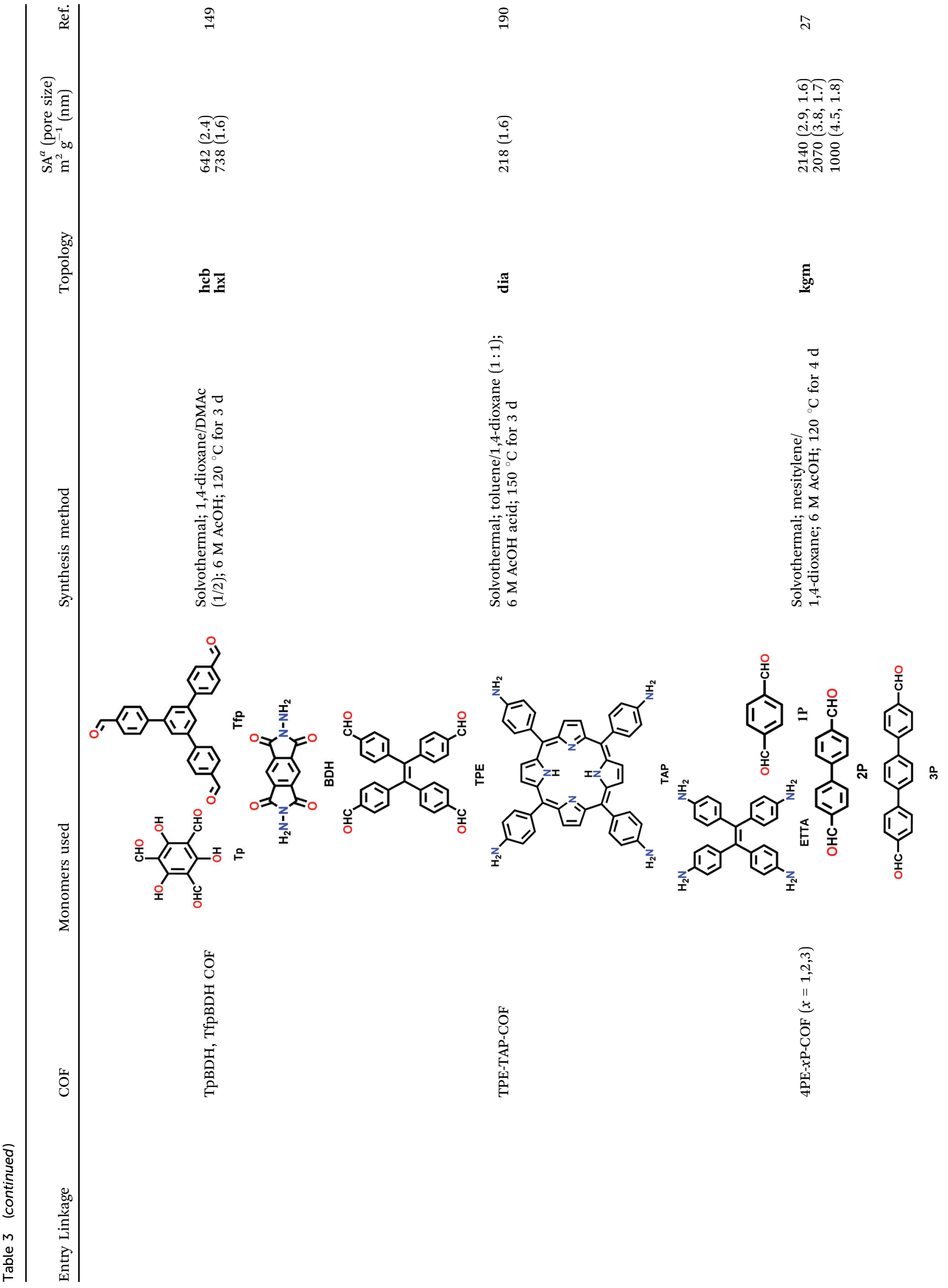




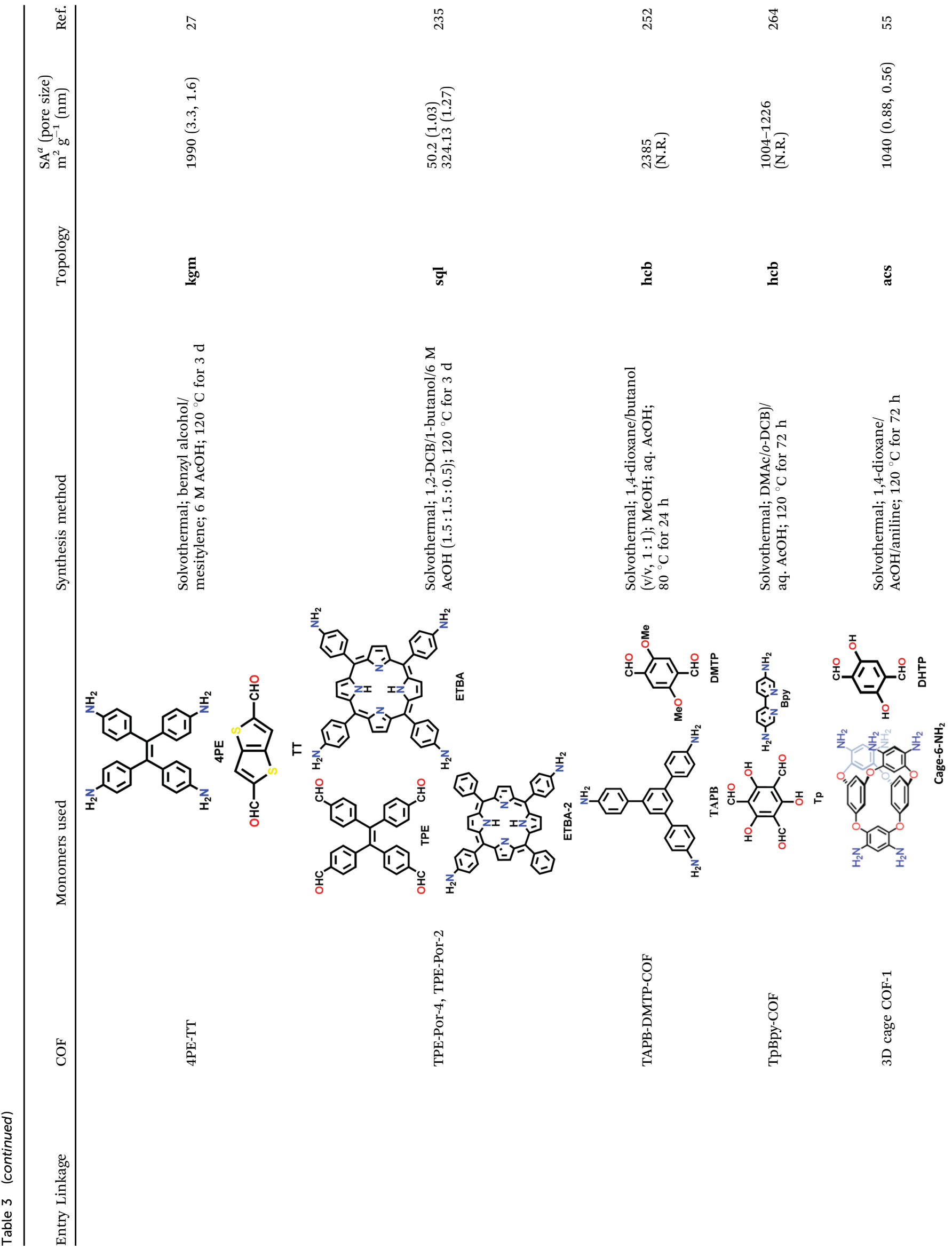



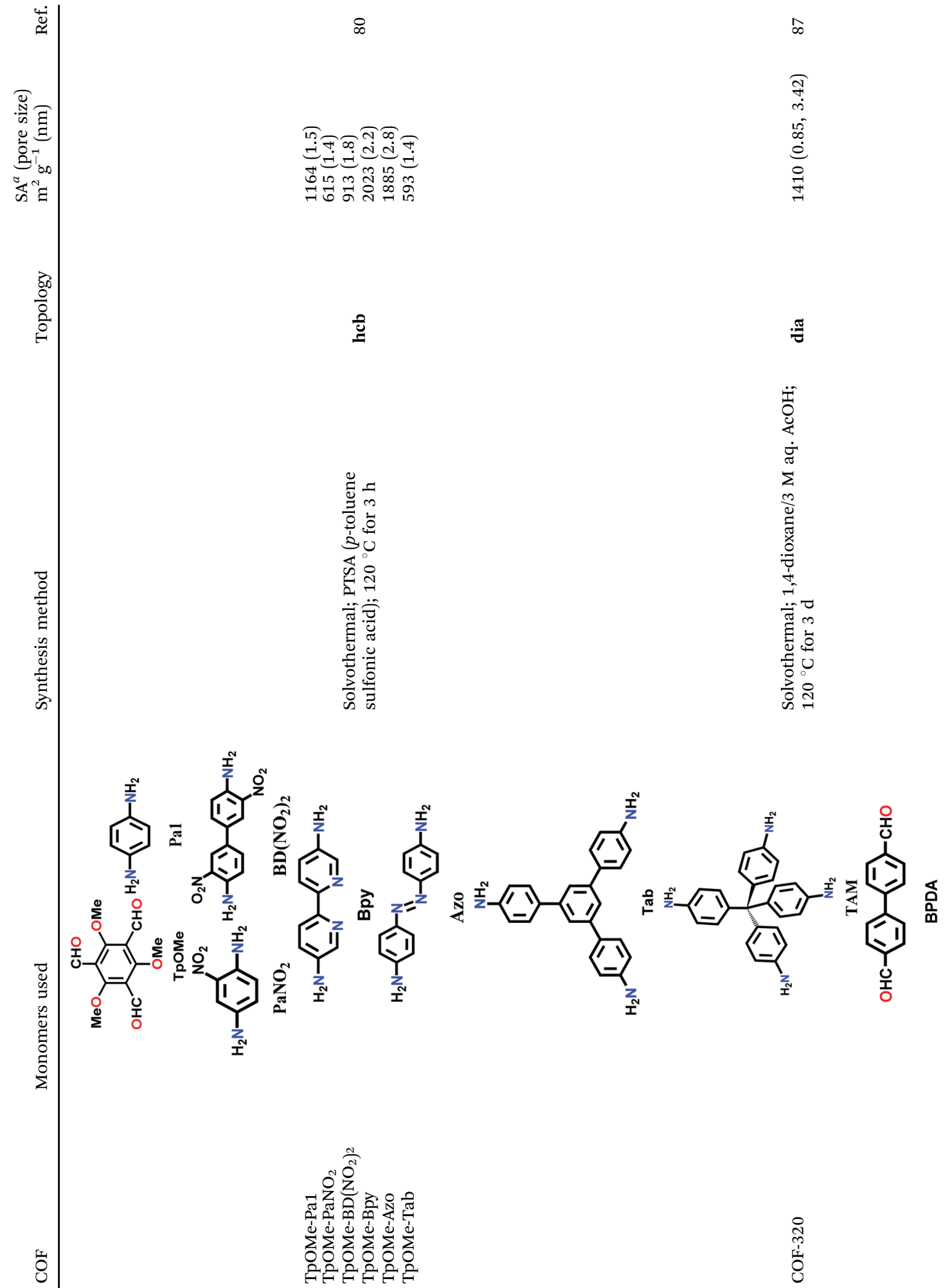

8
$\infty$
0
0
0
0 
Materials Advances

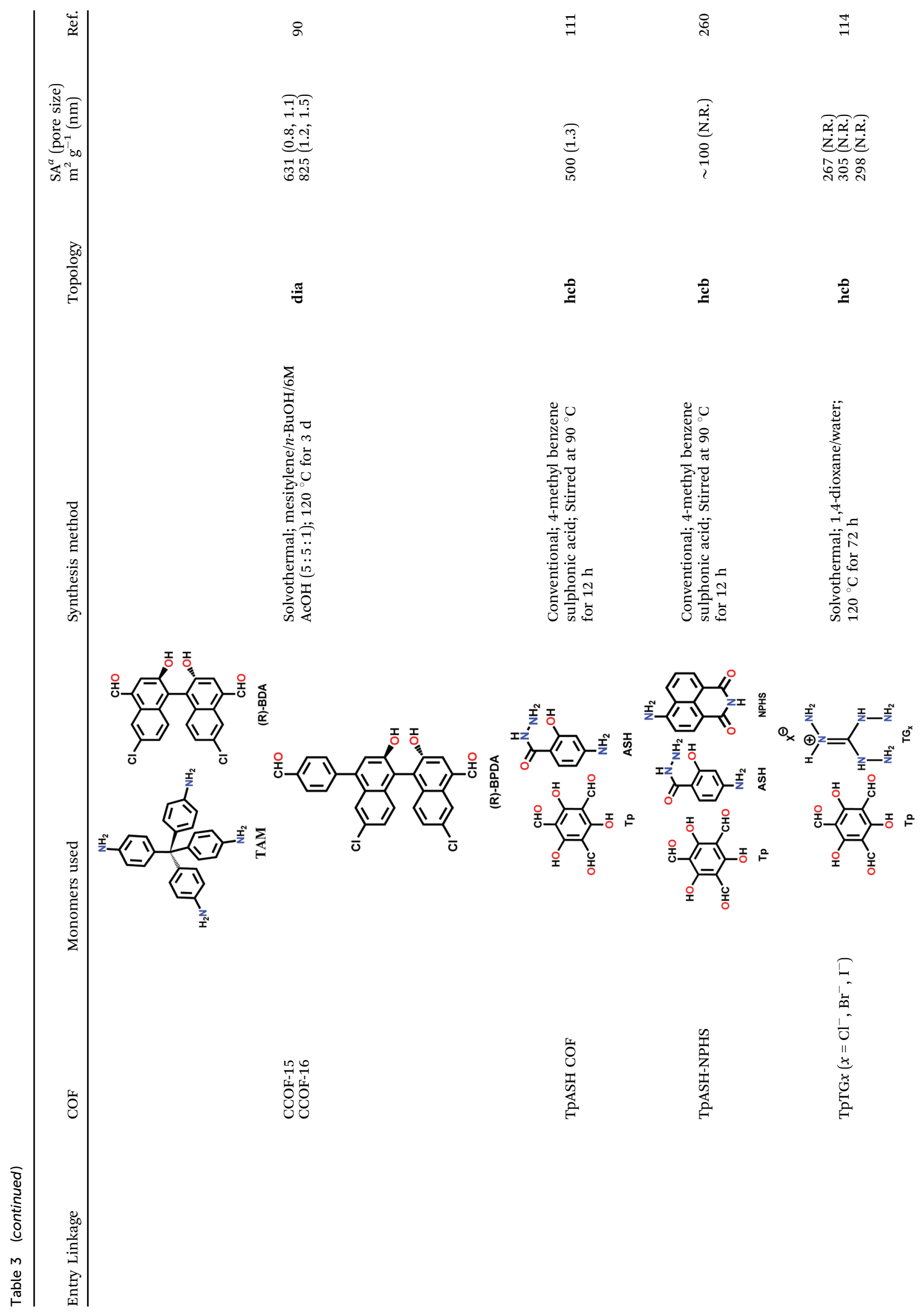




\section{Materials Advances}

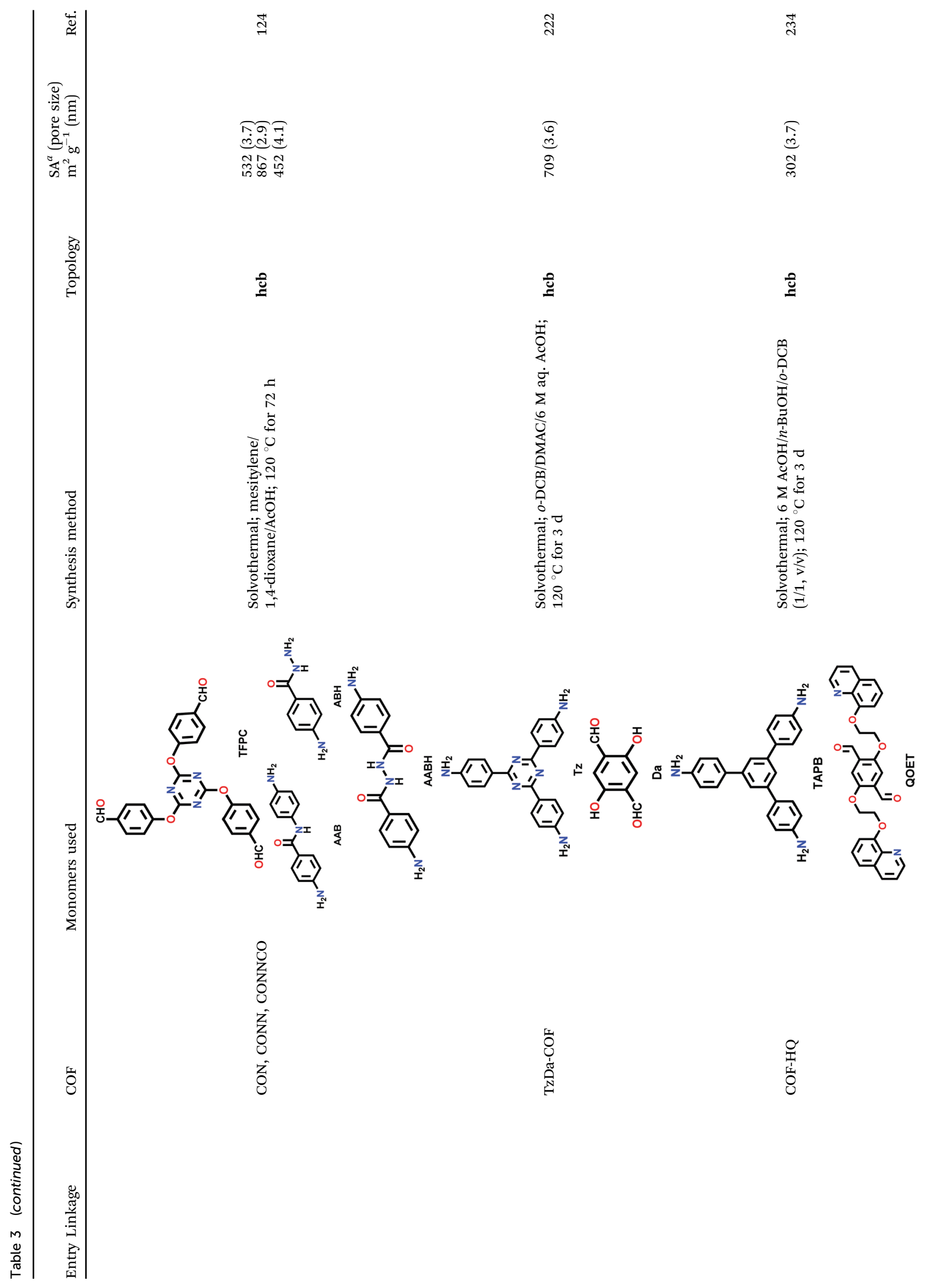


Review

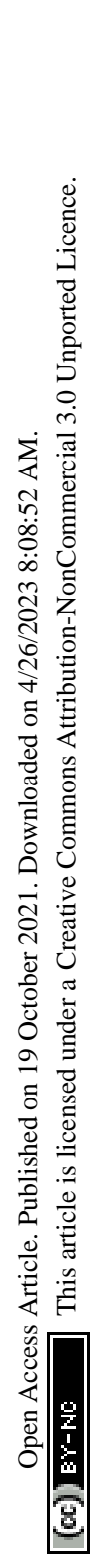

$\stackrel{ }{\sim}$

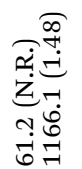

욜

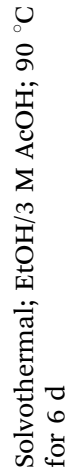

국

$\underset{\substack{\infty \\ \stackrel{3}{3}}}{\stackrel{0}{=}}$

๕

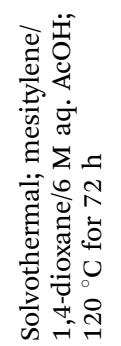

Materials Advances

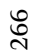

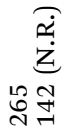

$\underset{\overbrace{}}{\stackrel{q}{q}}$

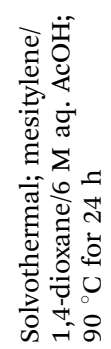

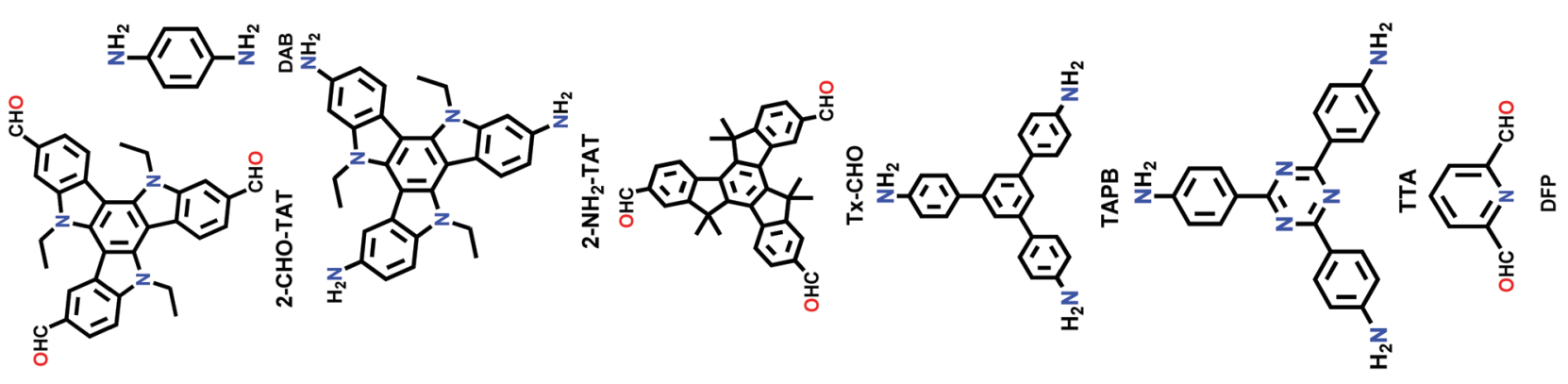




\section{Materials Advances}

View Article Online

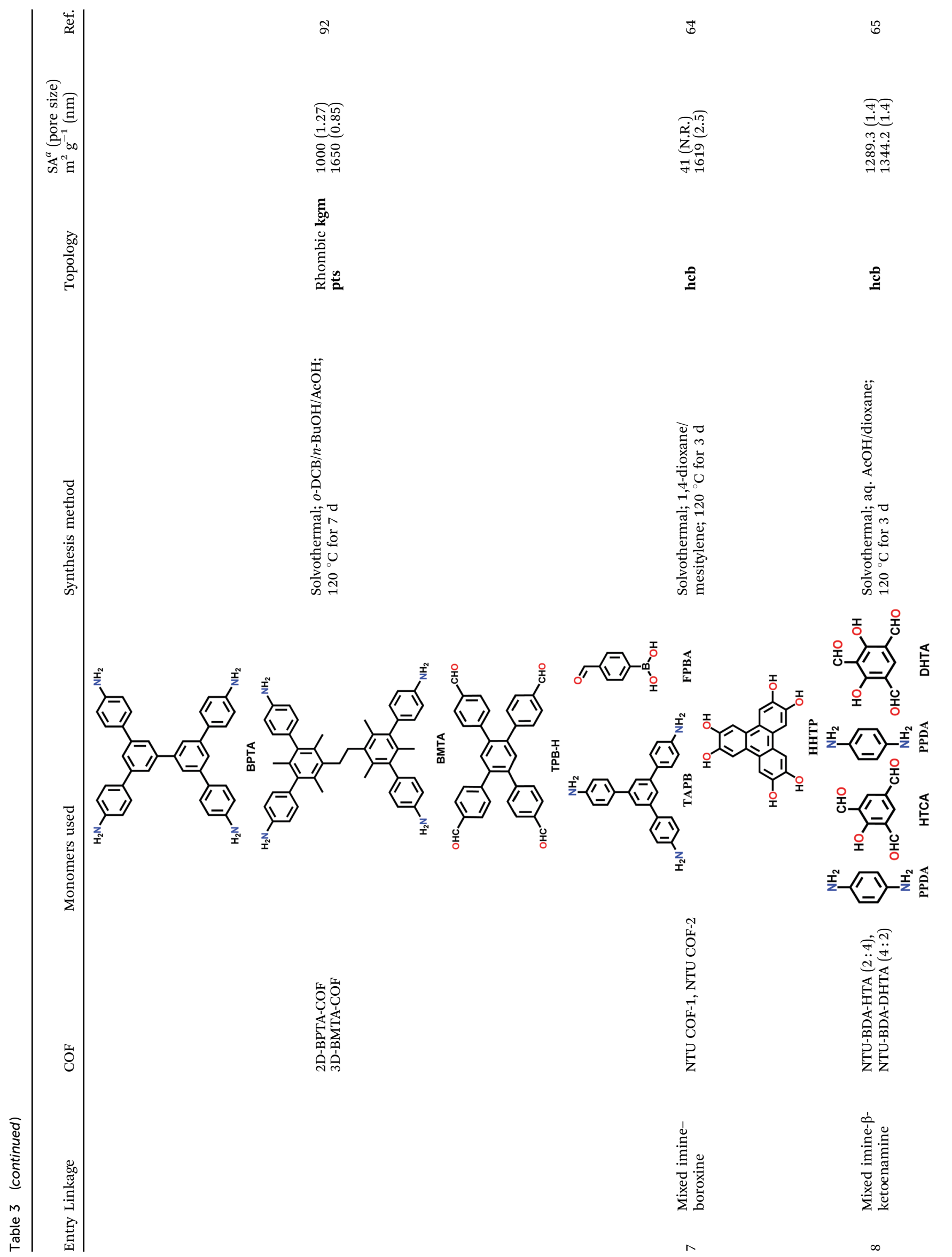

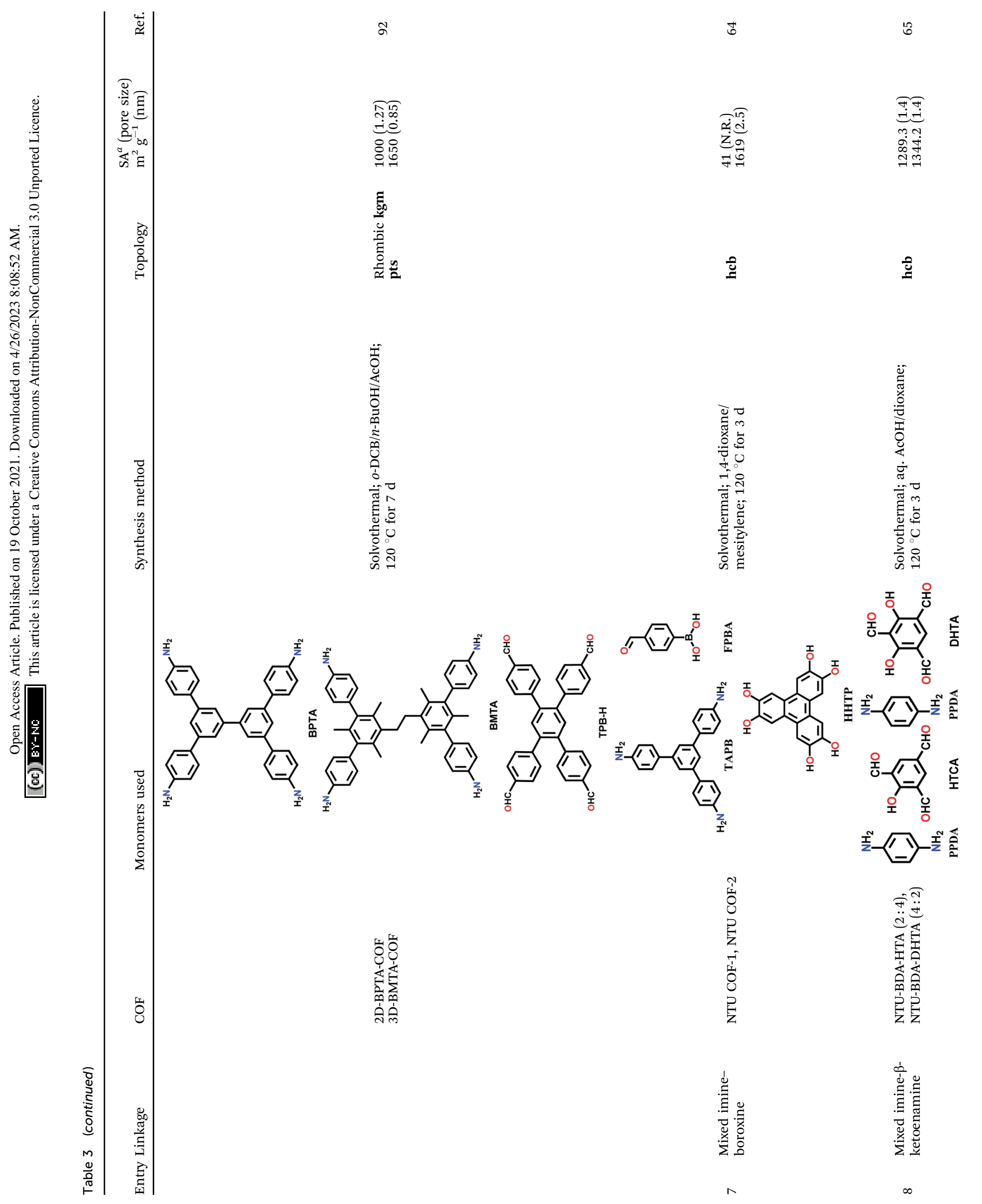




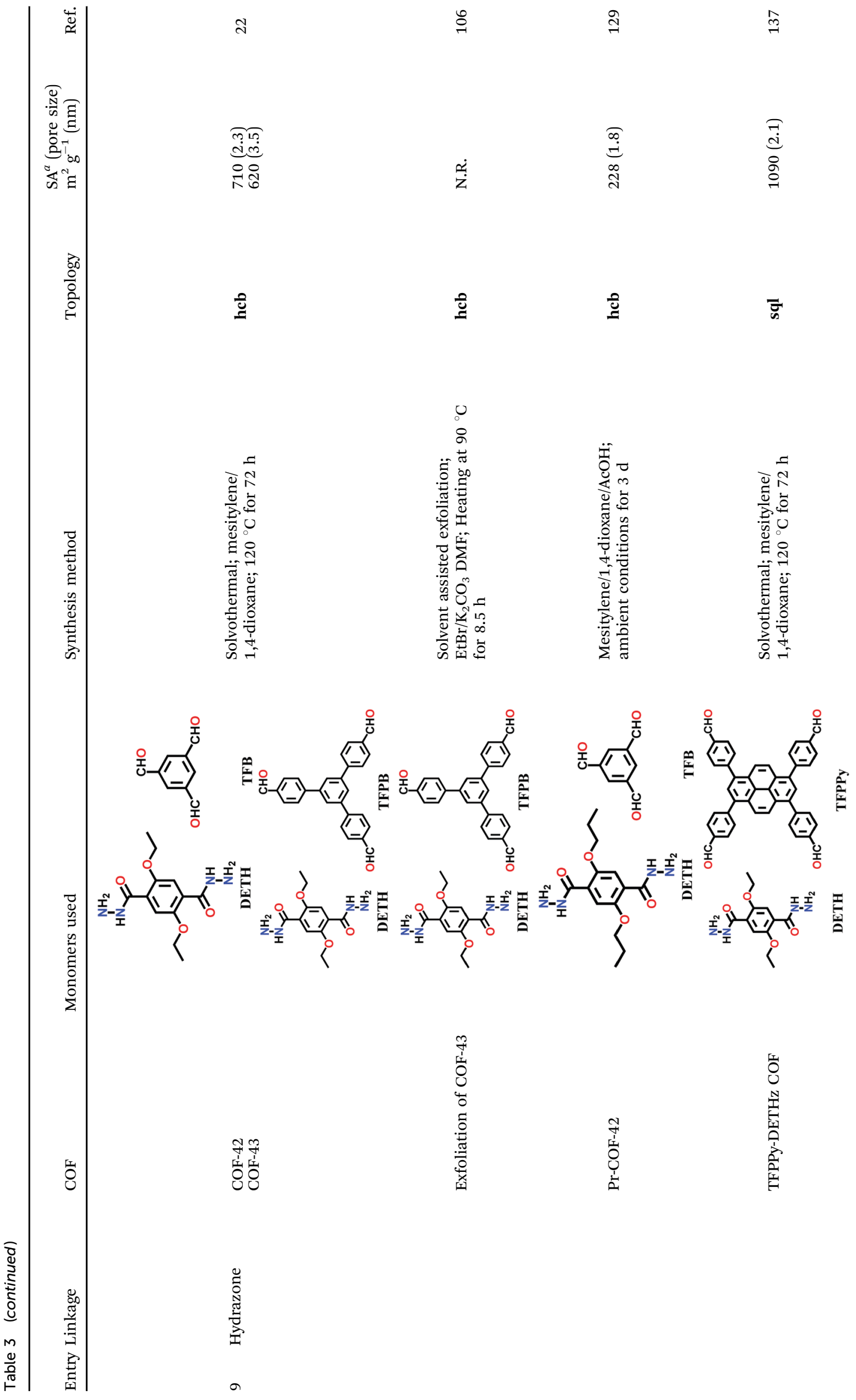




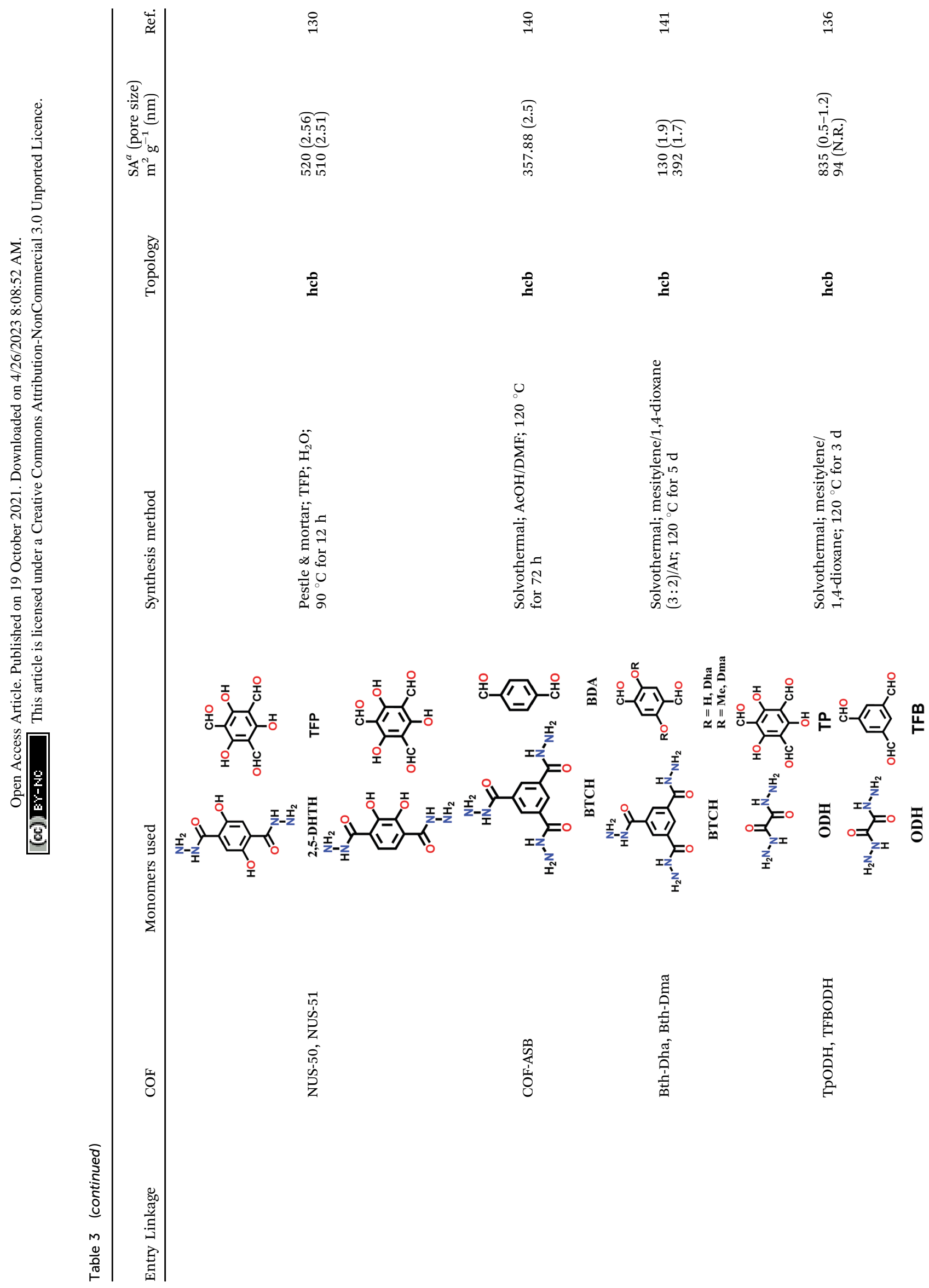




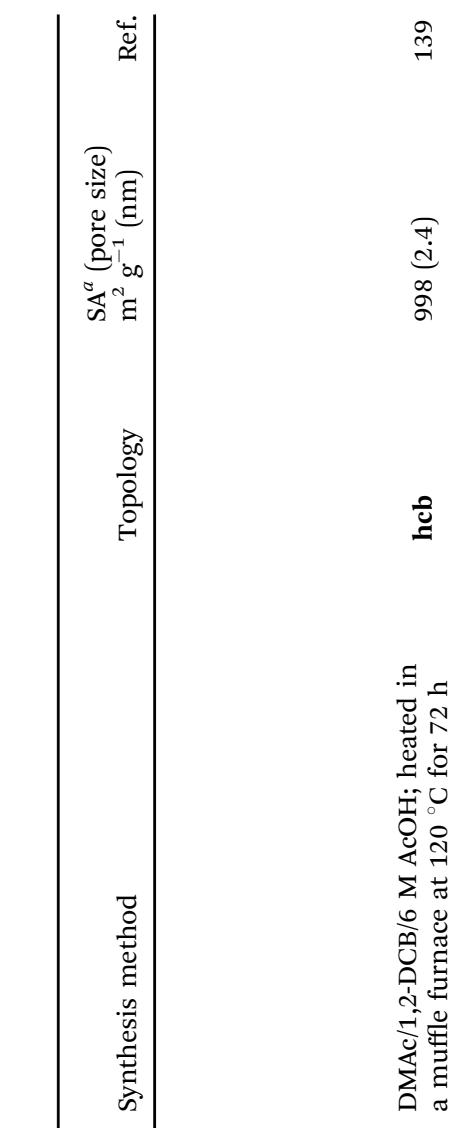

$\overrightarrow{\underline{9}}$

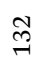

Materials Advances

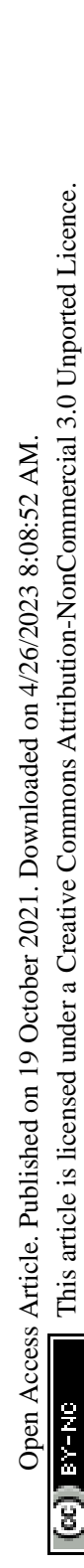

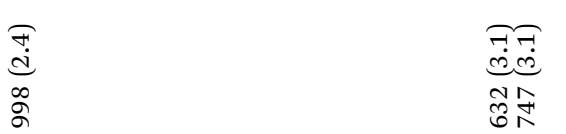

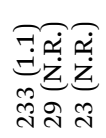

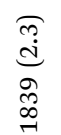

욜

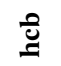

$\stackrel{0}{\Xi}$

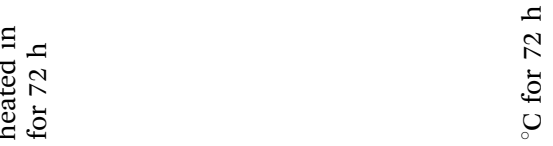

$\begin{array}{lll}5 & 0 & 0 \\ & 0 & 0 \\ 0 & 0 & 0 \\ 0 & 0 & 0 \\ 0 & 0 & 0\end{array}$

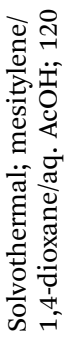

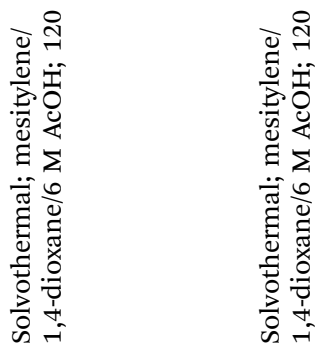

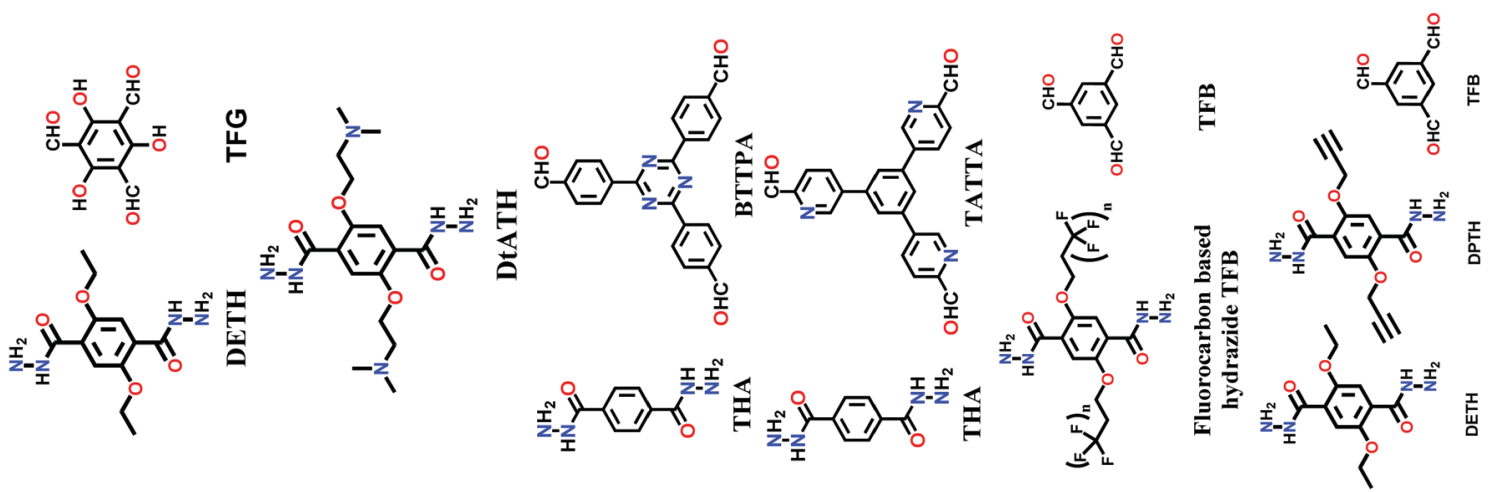

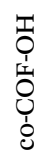

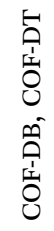

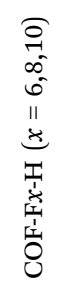

章

$\stackrel{\infty}{\rightarrow}$

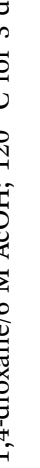


Materials Advances

View Article Online

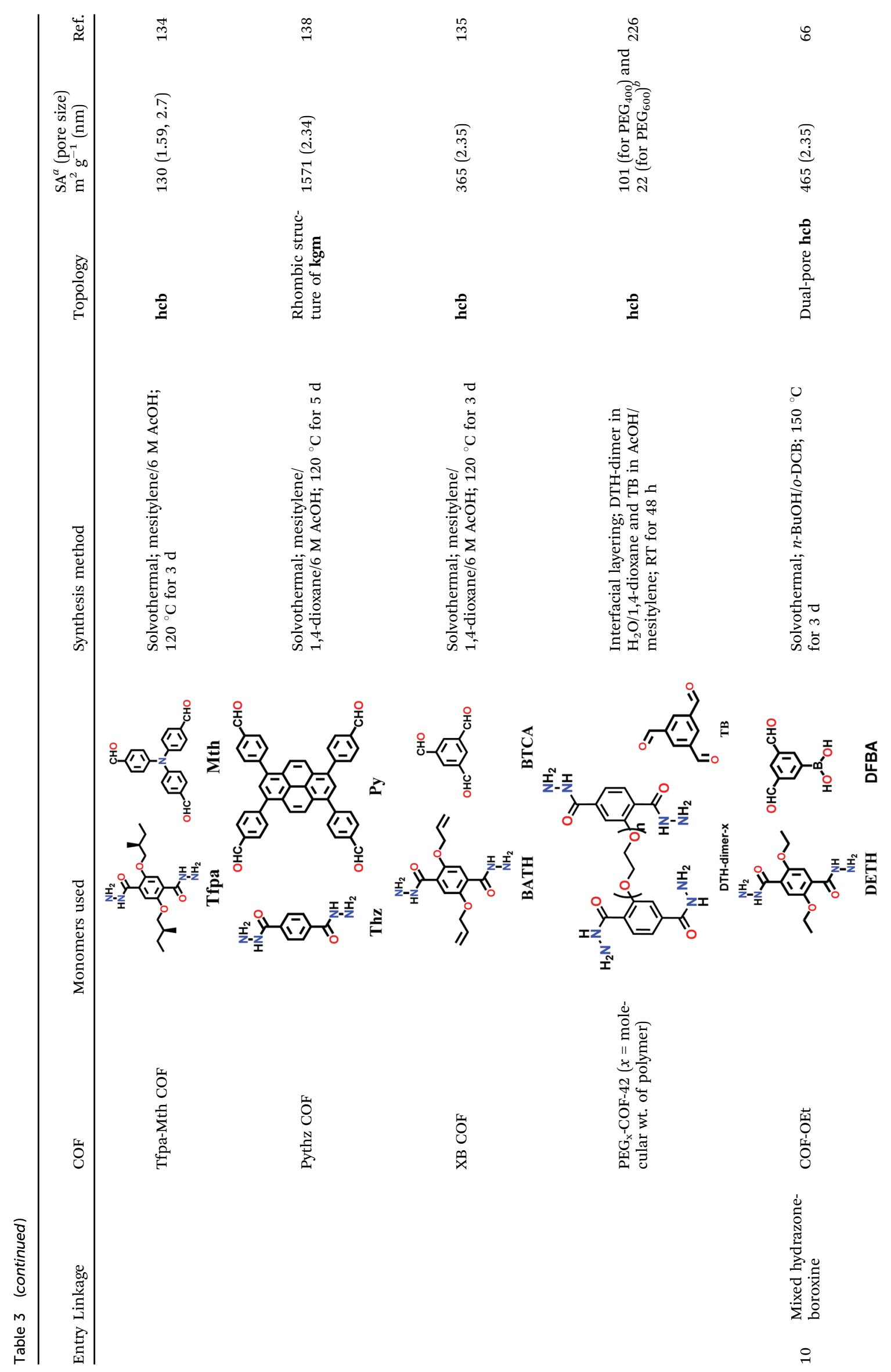




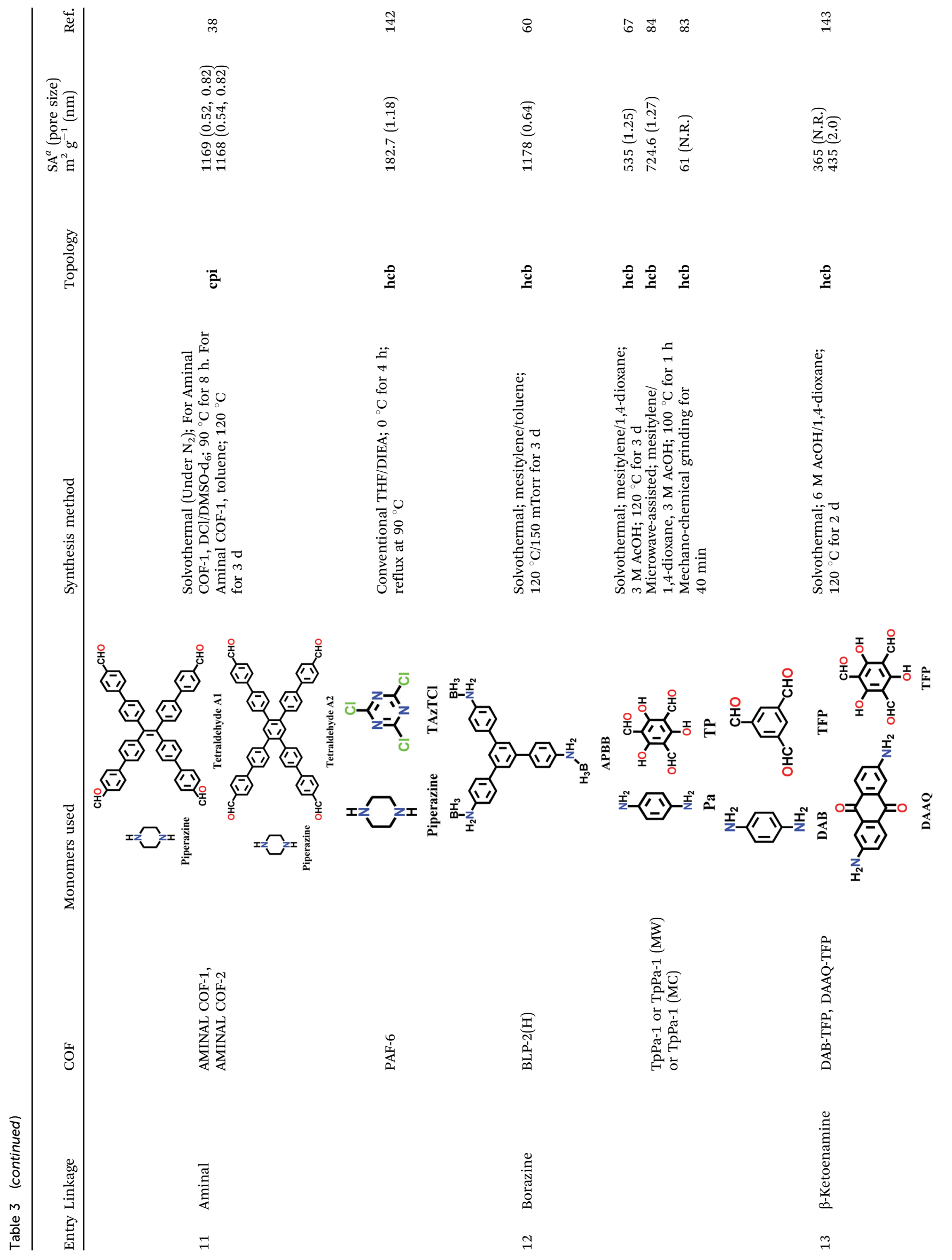


Materials Advances

View Article Online

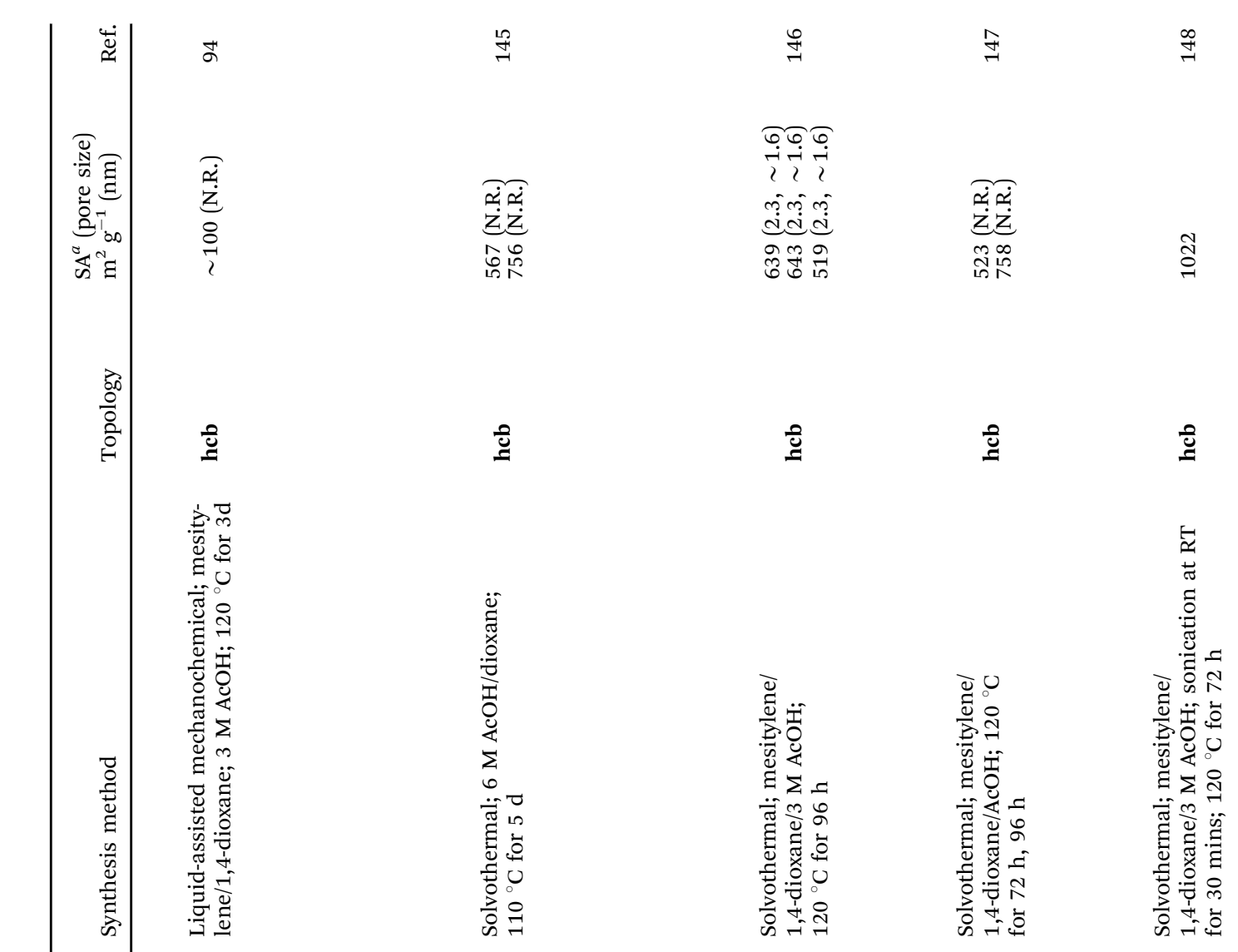

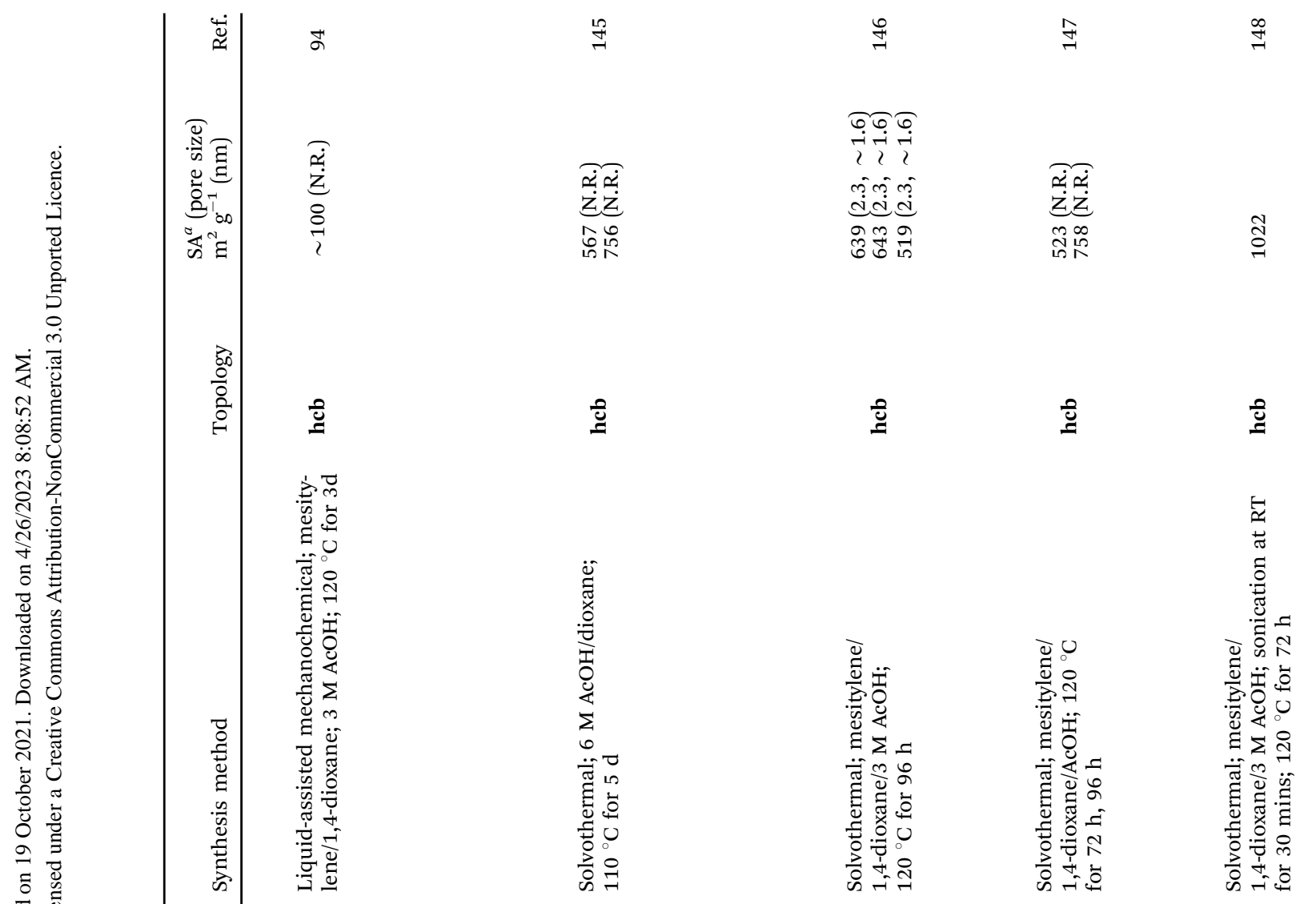

袋 (1)
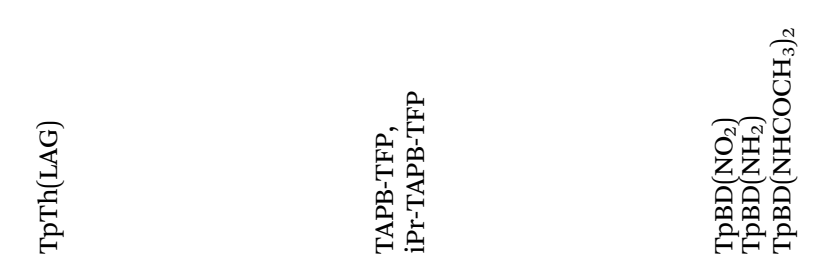

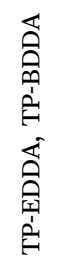

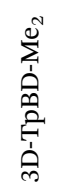




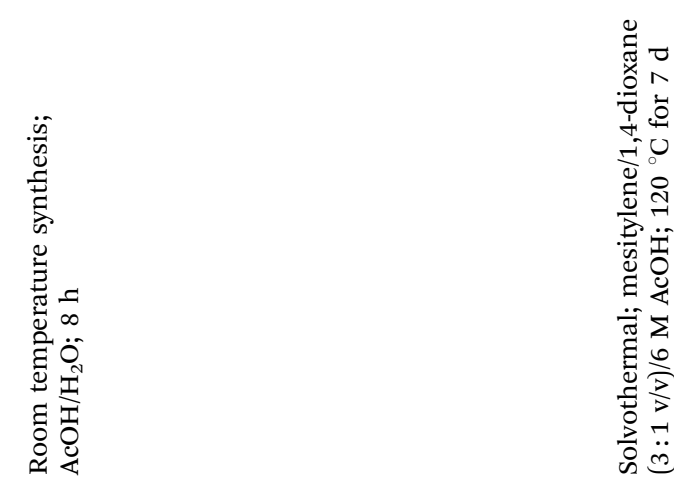

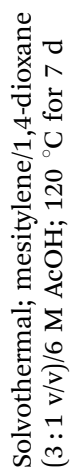

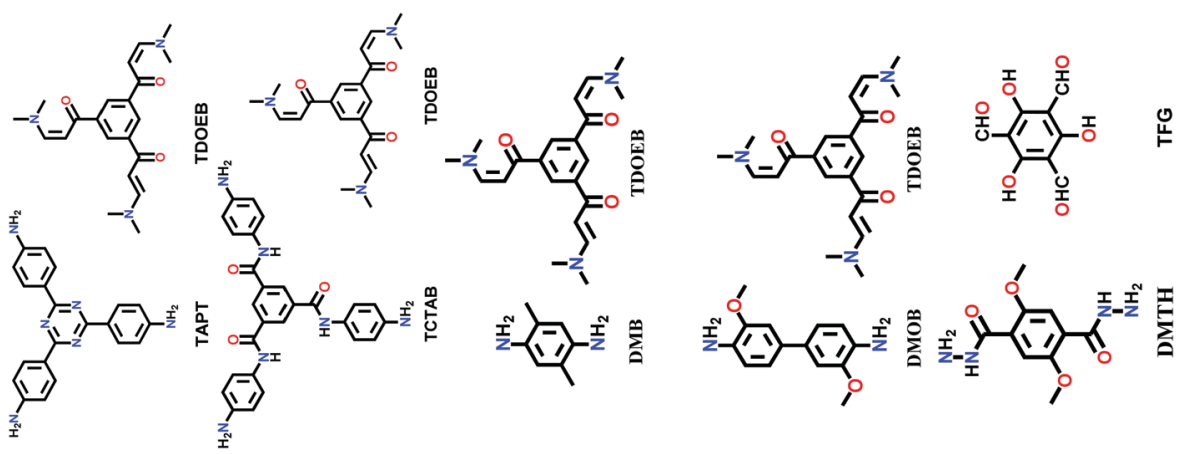




\section{Materials Advances}

View Article Online

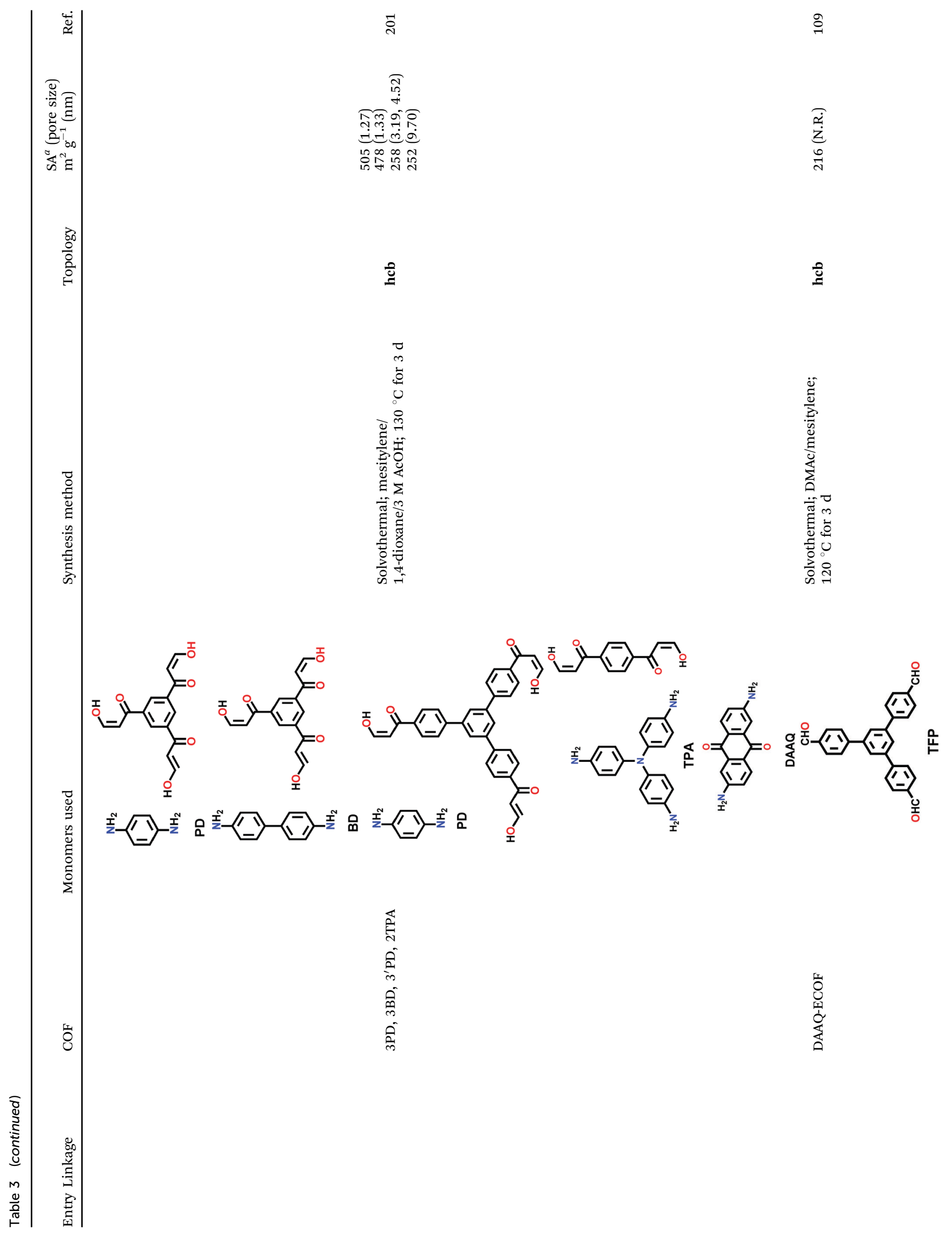


Review

Materials Advances

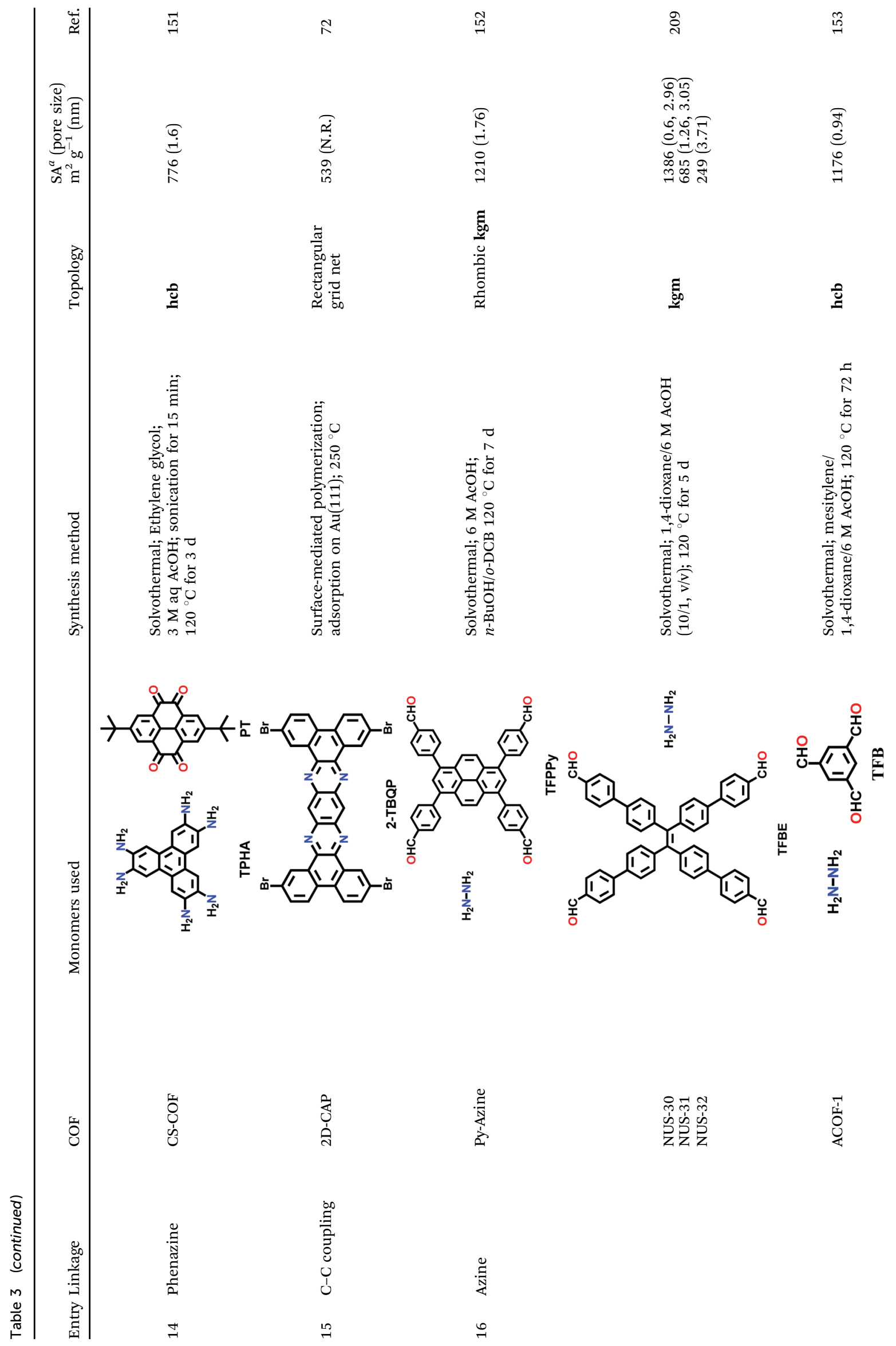


Materials Advances

View Article Online

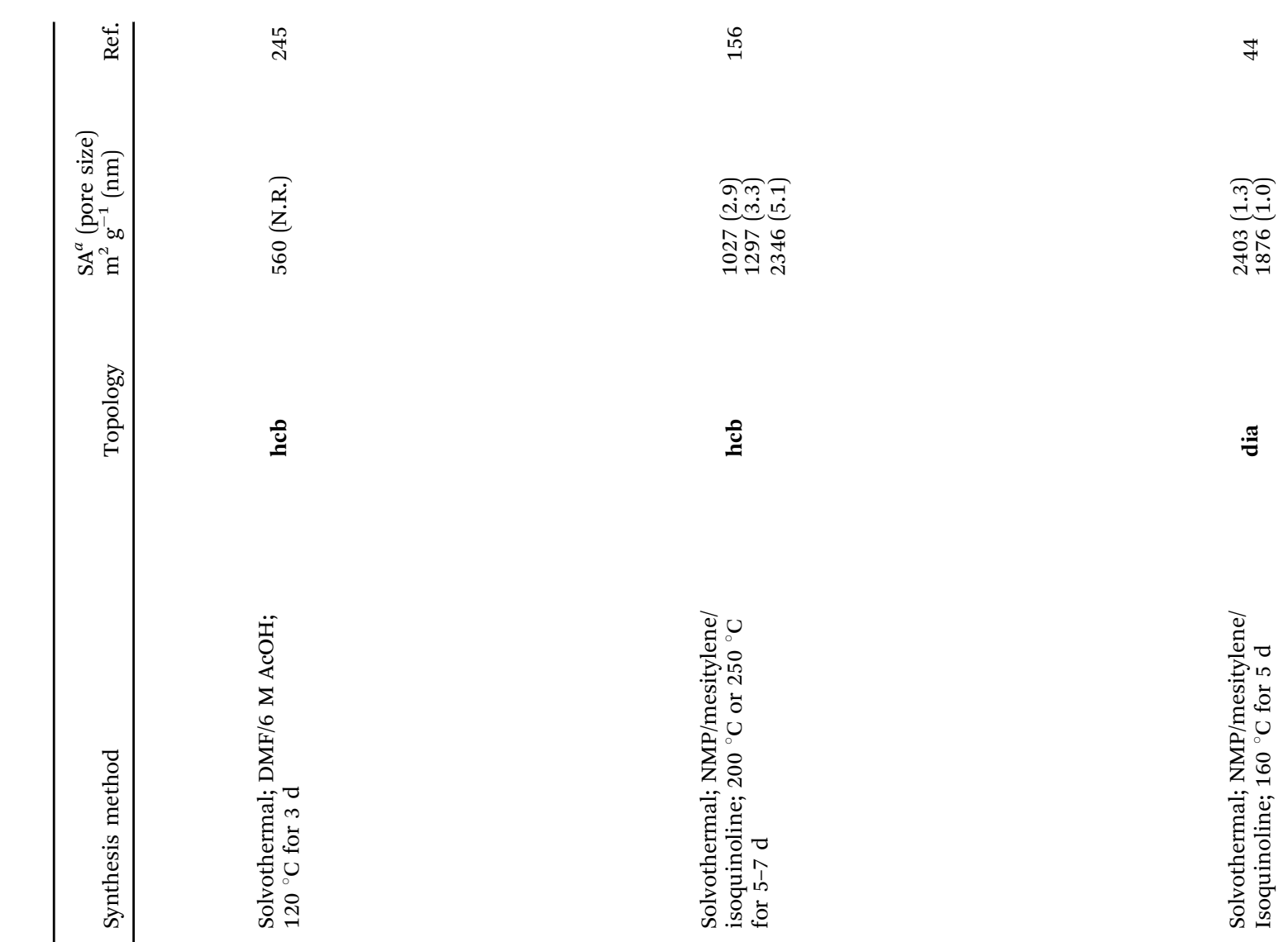

Review

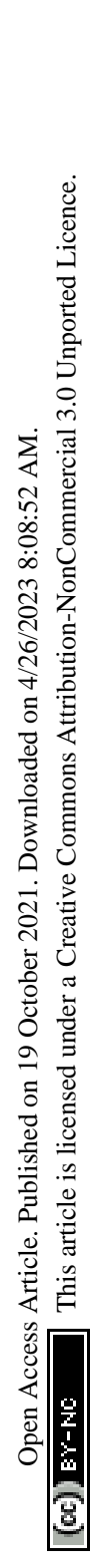

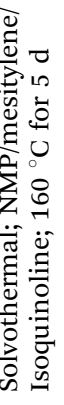

(n)

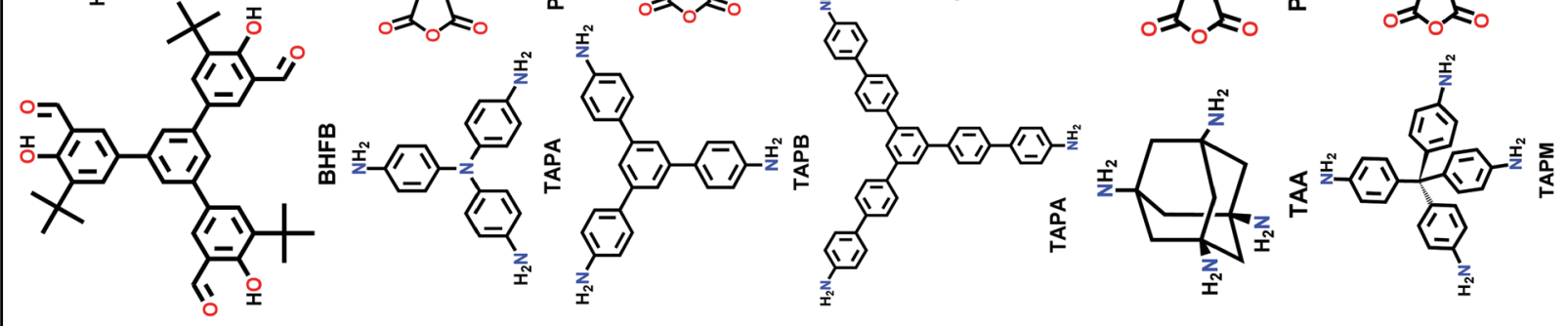

皆

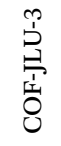

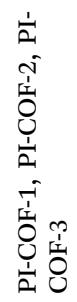

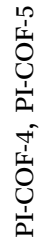

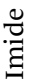

ન 


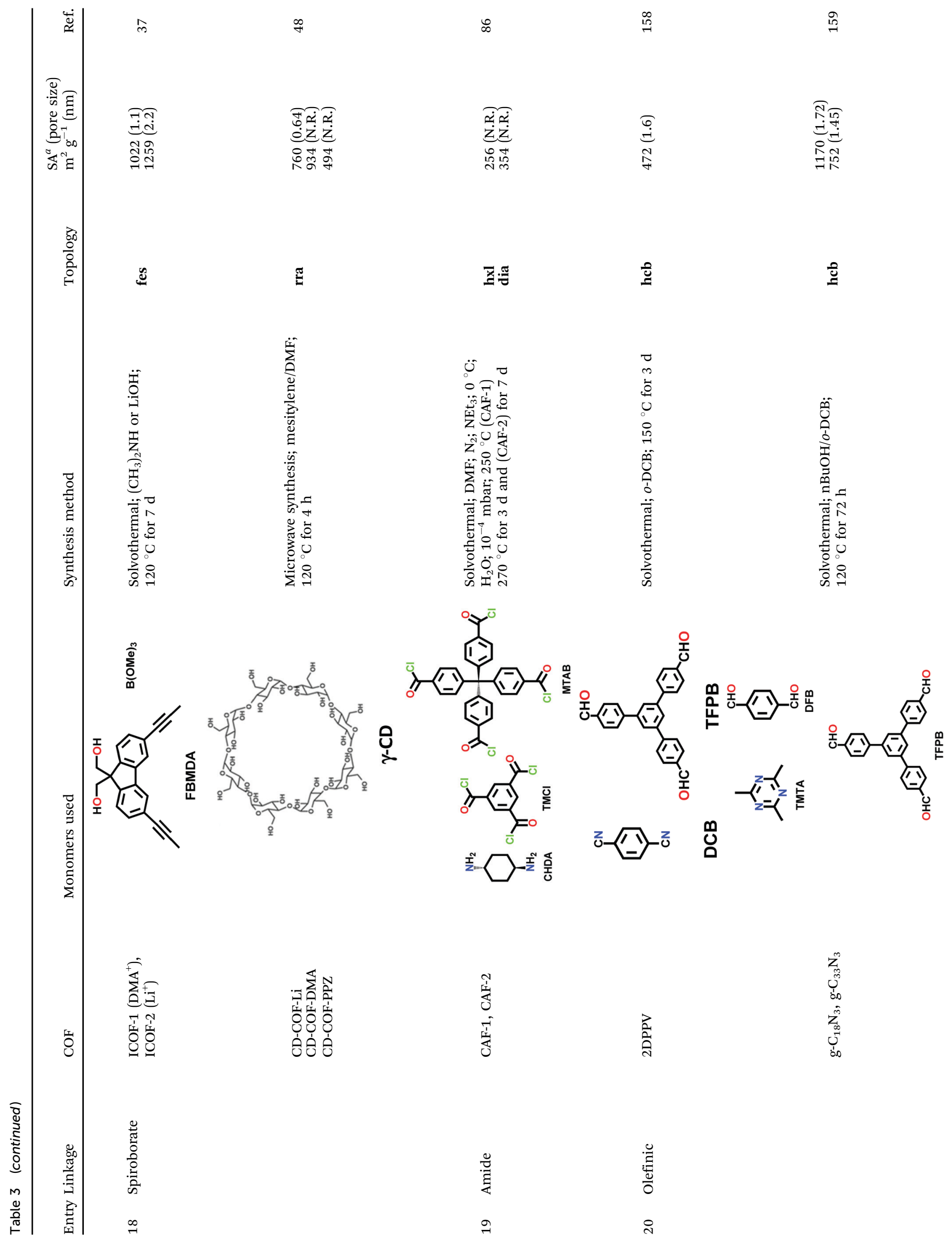


Materials Advances

View Article Online

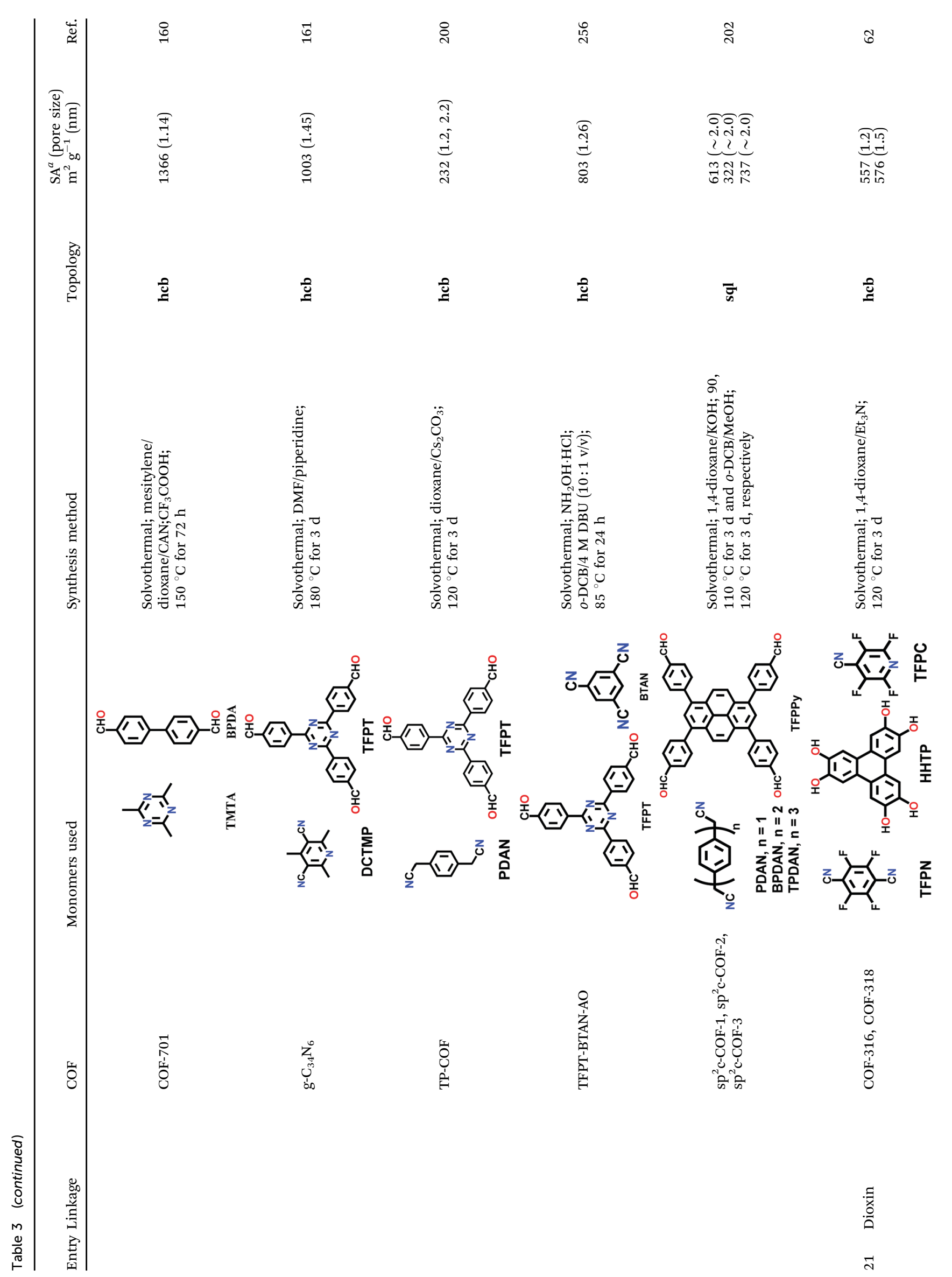




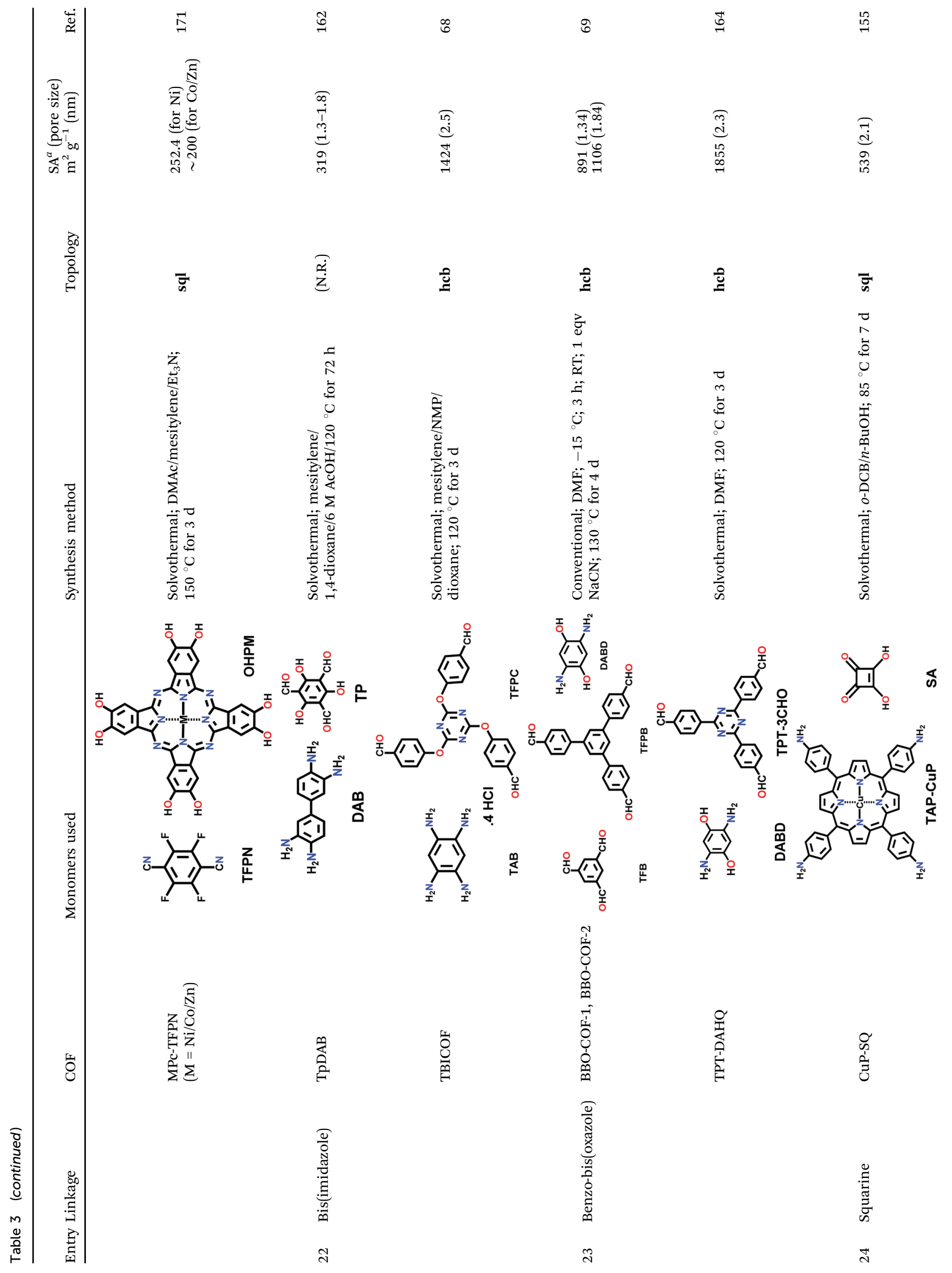




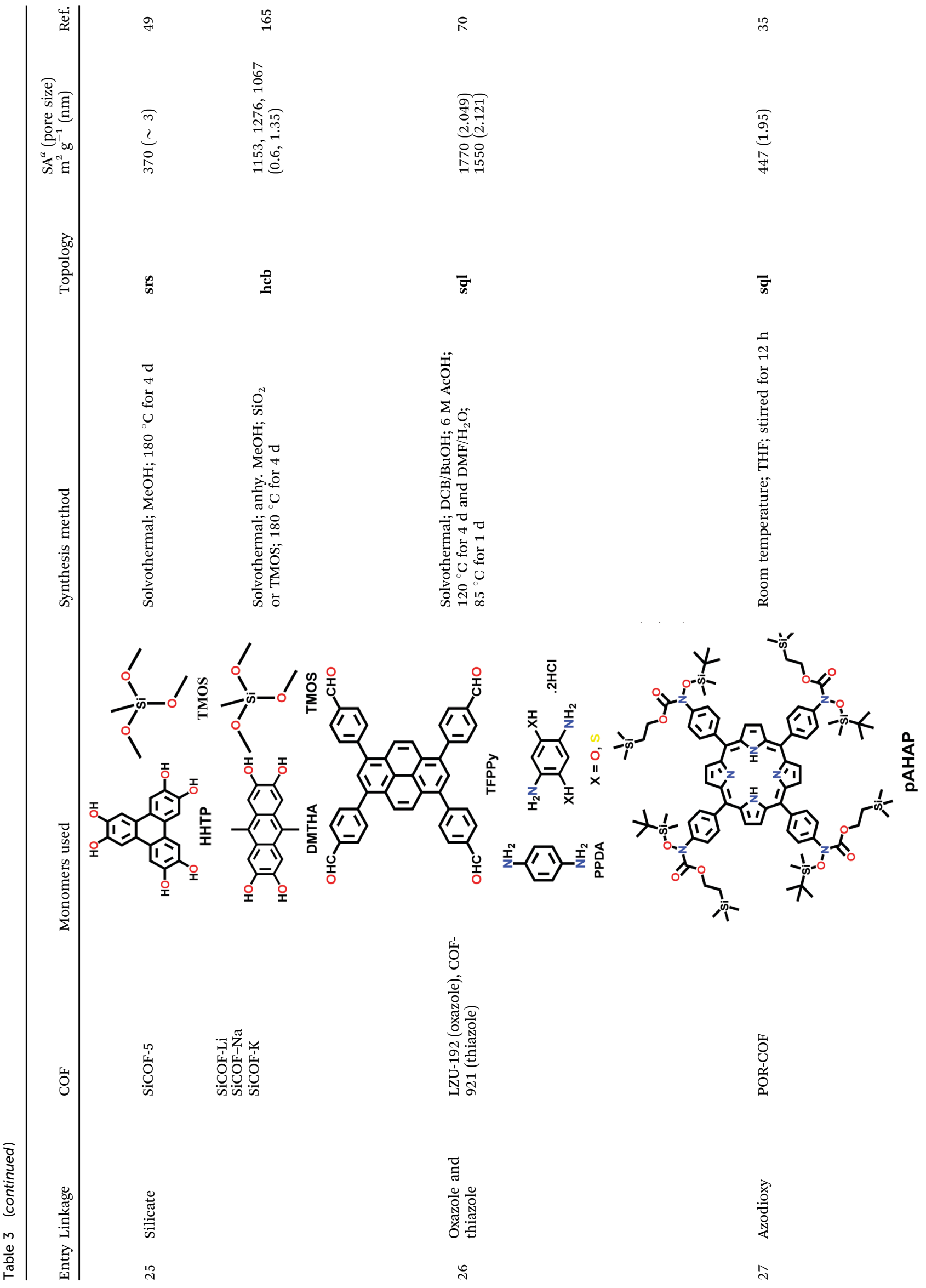




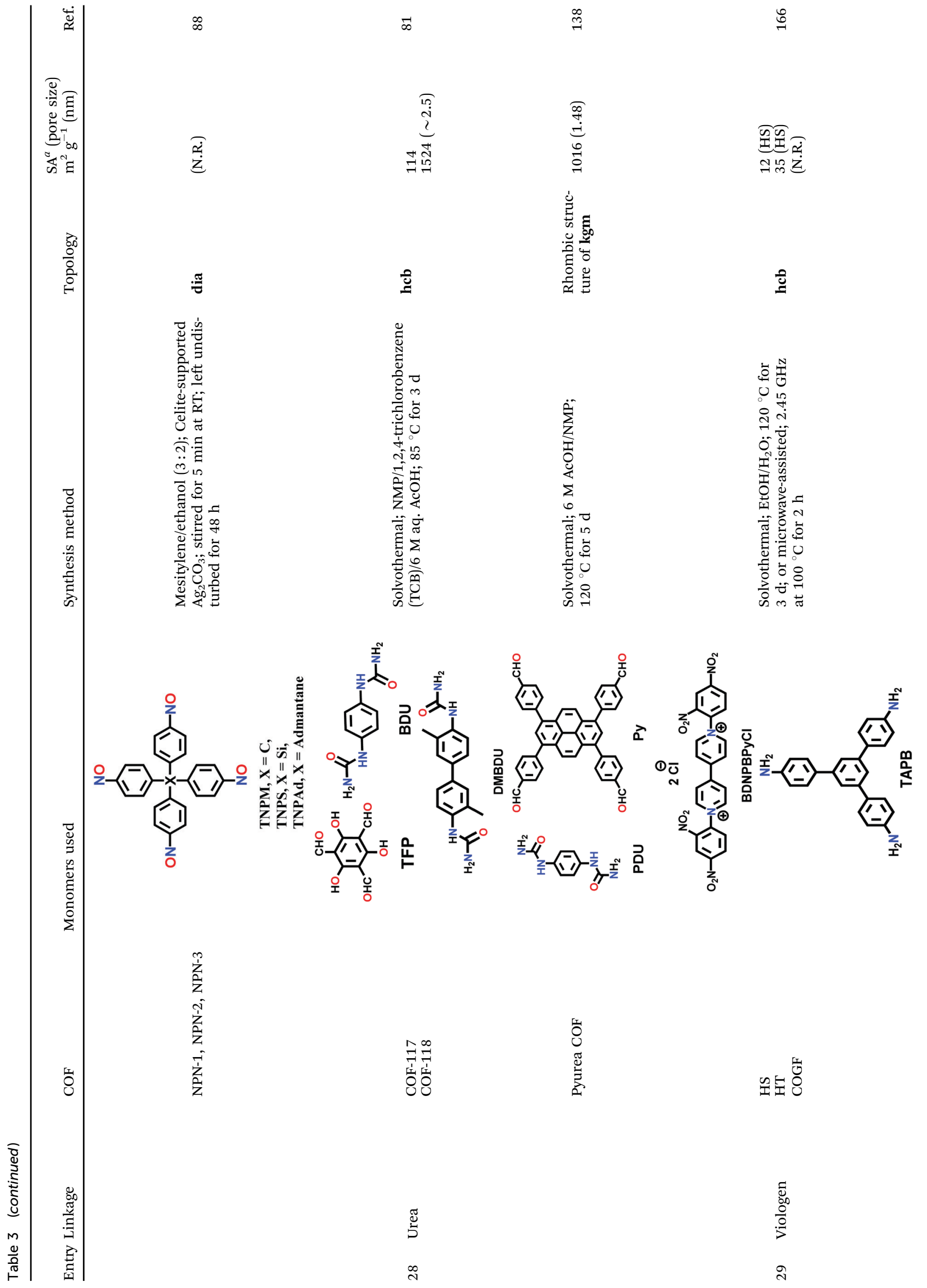


Materials Advances

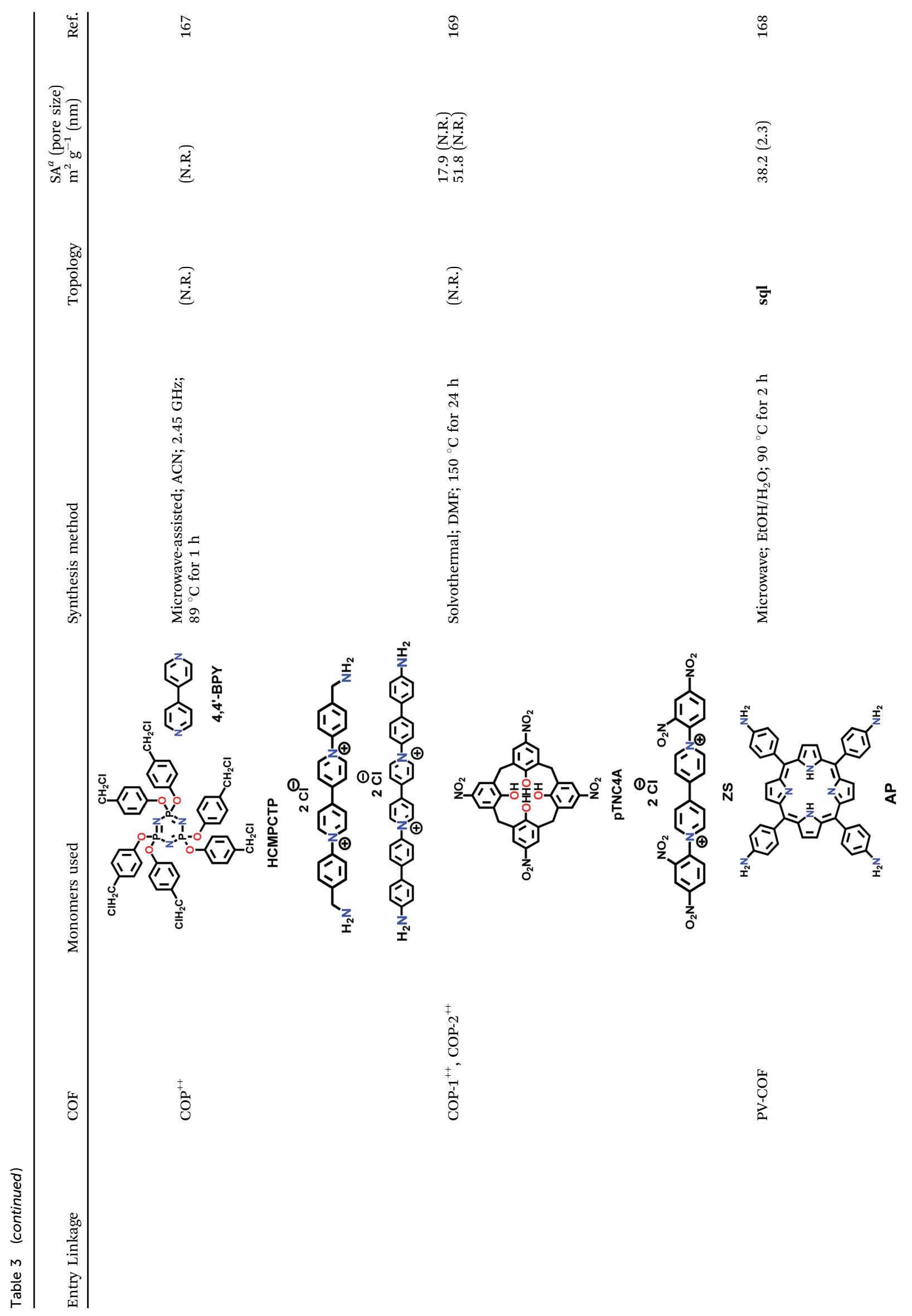




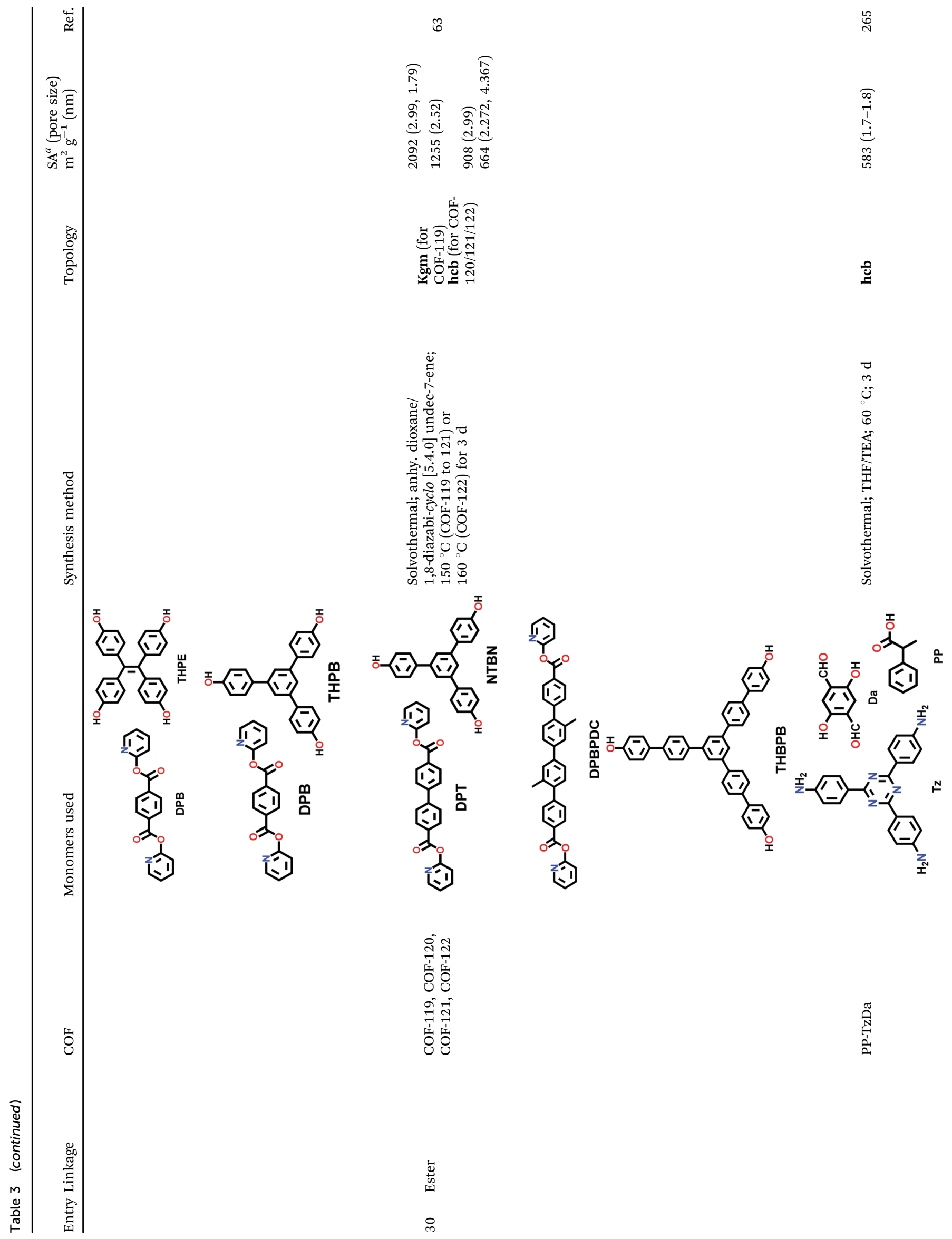




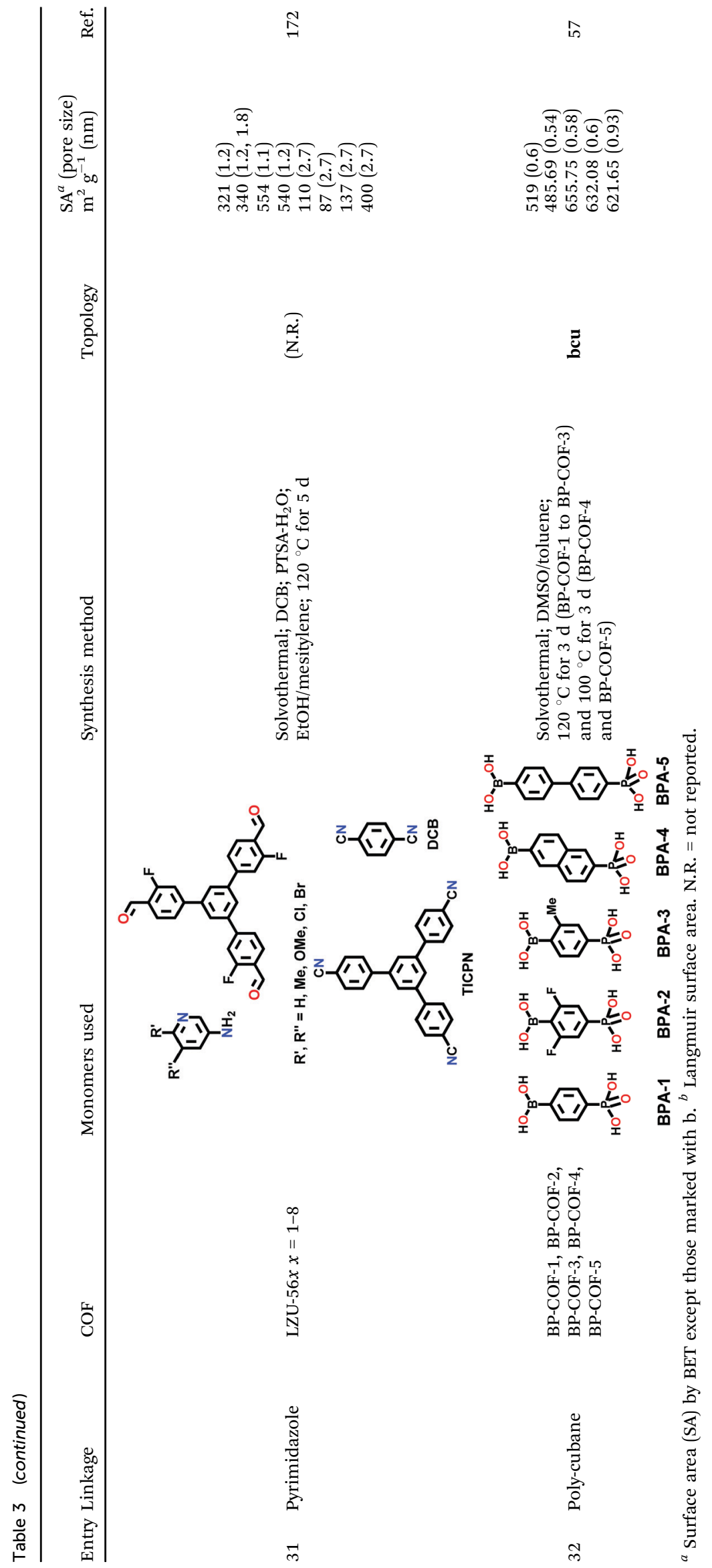




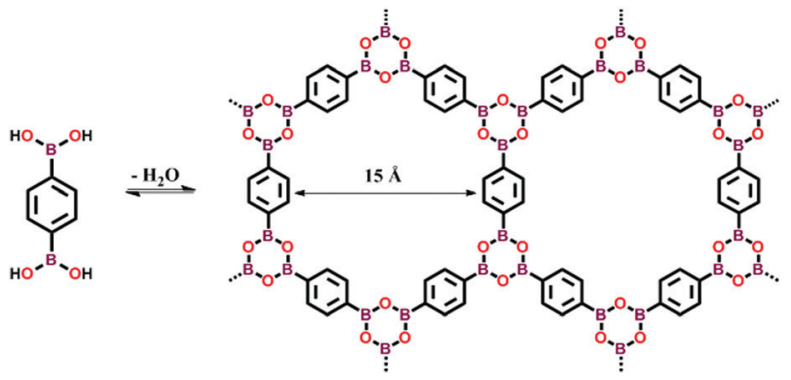

Fig. 8 Synthesis of boroxine-linked COF-1 via the self-condensation of 1,4-benzenediboronic acid. Adapted from ref. 4, Copyright The American Association for the Advancement of Science (2005).

inside its framework with more cross-linked density in comparison to CTF-1, and therefore, is more thermally stable up to $600{ }^{\circ} \mathrm{C}$ under an $\mathrm{O}_{2}$ atmosphere.

The other covalent triazine frameworks synthesized using the ionothermal technique include FCTF-1, FCTF-1-600, $m$-CTF and PTF-series. ${ }^{120,121}$ The FCTF series involves the inclusion of fluorine atoms in the framework, which improved the network stability and increased the number of ultramicropores $(<0.5 \mathrm{~nm})$. Alternatively, the $m$-CTF and PTF-series were subjected to molten $\mathrm{ZnCl}_{2}$ at different temperatures, i.e., $400{ }^{\circ} \mathrm{C}$ for the $m$-CTF series and 550-700 ${ }^{\circ} \mathrm{C}$ for the PTF series, with 1,3,5-tricyanobenzene (TCB) precursor.

$\mathbf{C}=\mathbf{N}$ linkage (imine). An imine bond is a dynamic covalent bond, which is usually synthesized via a reversible Schiff-base condensation reaction between amines and aldehydes. The imine-linked porous frameworks are N-rich and have higher thermal, chemical and hydrolytic (in some cases) stability compared to boron-containing frameworks. Imine-linked crystalline $\mathrm{COF}$ powders have been synthesized via solvothermal, mechanochemical, ionothermal and room-temperature synthesis. The first imine-linked 3D COF was formed in 2009 via the solvothermal condensation of a $T_{\mathrm{d}}$-symmetric tetra-(4-anilyl)methane (TMA) with the linear terephthaldehyde (BDA), as shown in Fig. 9a. ${ }^{82}$ Single crystals of COF-300 were obtained nine years later for structure determination by the single-crystal X-ray diffraction (SCXRD) technique (vide infra). ${ }^{6}$ The representation of COF-300 is [(TAM)(BDA $\left.)_{2}\right]_{\text {imine }}$ with seven-fold interpenetration. Also, the highly crystalline 2DILCOF-1 was synthesized at $120{ }^{\circ} \mathrm{C}$ via the condensation reaction between 1,3,6,8-tetrakis $(p$ formylphenyl)pyrene (TFPPy) and $p$-phenylenediamine (PDA) in 1,4-dioxane in the presence of aqueous acetic acid over 3 days (Fig. 9b). ${ }^{122}$ This COF had a larger surface area than that of the 3D imine-linked IL COF-1 (refer to Section 5.6).

The other COF powders with imine-linkages fabricated via the solvothermal technique include TFPC-NDA COF, ${ }^{24} \mathrm{JUC}-508$, JUC-509, and JUC-509-Y (Y = Mn, $\mathrm{Cu}$ and $\mathrm{Eu}),{ }^{123}$ and some single-crystal COFs include COF-303, LZU-79 and LZU-111 with dia and lon networks. ${ }^{6}$ The modulator-directed imine formation reaction was conducted, which increased the state of reversibility and provided a chance for error-correction. Aniline is a mono-functional molecule that hinders nucleation and promotes crystal growth. Two other single crystalline COFs, viz.,
COF-303 and LZU-79, were synthesized solvothermally via the reticular strategy and possessed very highly interpenetrated dia-c7 and dia-c10 topology, respectively. Alternatively, the combination of two $T_{\mathrm{d}}$-symmetric monomers tailored the LZU-111 COF with the distinct lon-b-c3 topology (vide supra, Section 2.2).

A series of unique semi-flexible and flexible imine-linked 2D COFs, namely CON, CONN and CONNCO, was solvothermally synthesized with high crystallinity and stability in water and at different $\mathrm{pH}$ (Fig. 10). ${ }^{124}$ The N-rich frameworks act as an efficient $\mathrm{ON}-\mathrm{OFF}$ pH-responsive proton scavenger (further discussed in Section 6.2.2).

High temperature-autogenous pressure conditions successfully worked for the synthesis of highly luminescent active, crystalline and stable COFs, namely the COF-N, COF-NN (Fig. 11a) and TAT-COF series (Fig. 11b). ${ }^{125,126}$ These $2 \mathrm{D}$ frameworks were well explored for the fluorescence-based detection of small volatile organic molecules.

Furthermore, several imine-linked covalent organic thin films and nanosheets have also been fabricated using the exfoliation, interfacial and continuous flow methods. Surface COFs, namely SCOF-IC1 and SCOF-LZU1 ${ }^{101}$ and single-layered sCOF $(\mathrm{A}+\mathrm{B})$ and $\mathrm{sCOF}(\mathrm{C}+\mathrm{D}),{ }^{127}$ were constructed via solid/ vapour interfacial synthesis.

The formation of single-layered $\mathrm{SCOF}(\mathrm{A}+\mathrm{B})$ and $(\mathrm{C}+\mathrm{D})$ requires control of the conformation of the precursor during the reaction. Highly rigid, aromatic monomers were chosen to construct single-layered films at ambient conditions, whereas more flexibility of aliphatic monomers requires ultra-high vacuum (UHV) conditions after impinging on the Au(111) surface.

A unique method of continuous flow was employed for the synthesis of COF-LZU-1 from 1,3,5-triformylbenzene (TFB) and $p$-phenylenediamine (PPDA). ${ }^{116}$ The stock solution of both monomers was separately formed in a mixture of dioxane and acetic acid, and then injected together in a flow reactor at a rate of $20 \mu \mathrm{L} / \mathrm{min}$ under ambient conditions, yielding a bright yellow product.

Mixed triazine-imine $(\mathbf{C}=\mathbf{N})$ linkage. The only example in this category is TRITER-1, which was synthesized via a one-pot polycondensation reaction between 4 -aminobenzonitrile and terephthalic acid in the presence of triflic acid. ${ }^{128}$ The reaction mixture was refluxed at $150{ }^{\circ} \mathrm{C}$ for $12 \mathrm{~h}$ in the high boiling-point solvent, DMF. Initially, the in situ synthesis of 1,3,5-tris-(4aminophenyl)triazine (TAPT) was conducted using a nitrilesubstituted unit supported by acid-catalyzed trimerization. Subsequently, it underwent a Schiff base condensation reaction, yielding mixed triazine-imine linkages. This framework is N-rich, thermally stable, and possesses a high surface area and supermicropores (1.5 nm).

Mixed imine-boroxine or boronic ester $(\mathrm{C}=\mathrm{N}$ and $\mathrm{B}-\mathrm{O})$ linkage. Binary NTU-COF-1 and ternary NTU-COF-2 involve the formation of two types of covalent bonds (C-N and B-O). ${ }^{64}$ The orthogonal reaction strategy to construct NTU-COF-1 involves the use of 4-formylphenylboronic acid (FPBA) and 1,3,5-tris(4-aminophenyl)-benzene (TAPB). The bifunctional FPBA unit contains a boronic acid group on one end, which is easily converted into a boroxine ring under high thermal conditions, and an aldehydic 

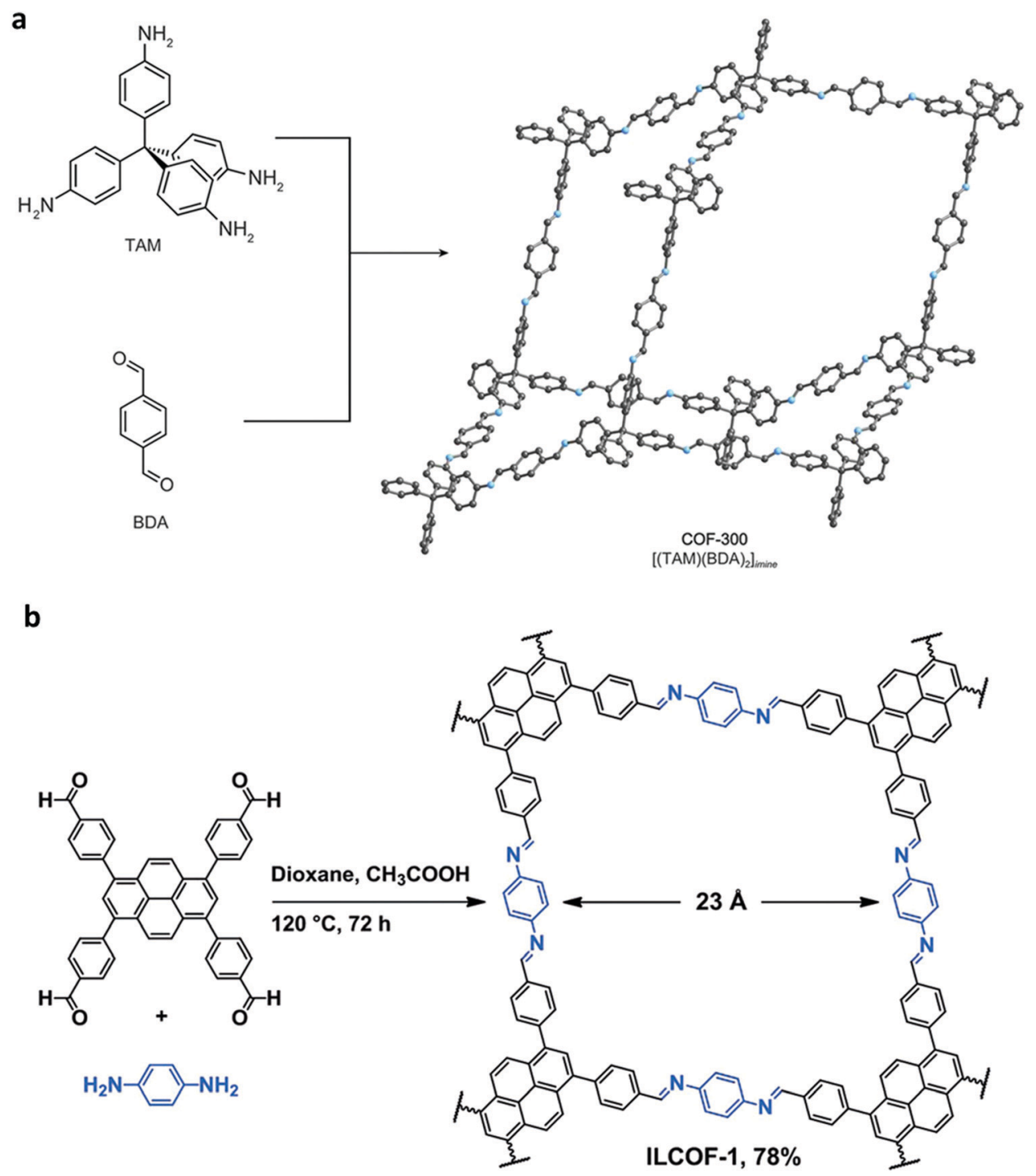

Fig. 9 (a) Synthesis of COF-300 and (b) 2D ILCOF-1. Reproduced with permission from: (a) ref. 6, Copyright The American Association for the Advancement of Science (2018) and (b) ref. 122, Copyright John Wiley and Sons (2013).

group on the other end, generating an imine-linkage. Both reactions are compatible and do not interfere with each other to carry out feasible orthogonal reactions. Similarly, NTU-COF-2 utilizes three different monomers, TAPB, FPBA and HНTP, in 1,4-dioxane solvent. Therefore, solvothermal conditions are employed for the synthesis of mixed imine-boroxine linked COFs, i.e., heating at $120{ }^{\circ} \mathrm{C}$ for $72 \mathrm{~h}$ for both NTU-COF-1 and NTU-COF-2.

Hydrazone ( $\mathrm{C}=\mathrm{N}-\mathrm{NH}_{2}$ ) linkage. A large number of covalentlybonded frameworks with hydrazone-linked monomeric units has been reported thus far. These frameworks are either formed as crystalline bulk powders and/or few-layered thin films. The first hydrazone-linked COFs were reported in 2011, which are mesoporous crystalline COF-42 and COF-43 constructed by the reversible dehydration reaction of 2,5-diethoxyterephthalohydrazide (DETH) and 1,3,5-triformylbenzene (TFB) or 1,3,5tris(4-formylphenyl)benzene (TFPB) in the presence aqueous acetic acid and a mesitylene/1,4-dioxane mixture in a flamesealed tube at $120{ }^{\circ} \mathrm{C}$ for $72 \mathrm{~h}$, respectively. ${ }^{22}$ Both COFs were collected as a pale-yellow microcrystalline powder. The few-layered COF-43 was obtained one year later by employing the solventassisted exfoliation technique and solid/vapour interfacial method. ${ }^{106}$ The appropriate choice of solvent yields COF-layered sheets with high aspect ratios. In addition to solvothermal conditions, the room-temperature synthesis of COF-42, COF-43 and Pr-COF-42 was performed in 2017 with the same monomers, DETH (propyl substituted for Pr-COF-42) and TFB. ${ }^{129}$ The monomers were suspended in a 1,4-dioxane/mesitylene mixture in the presence of $6 \mathrm{M}$ aq AcOH catalyst and then left undisturbed for 3 days. Highly crystalline frameworks were obtained, particularly COF- 42 with $72 \%$ yield, which was found to be comparable to that synthesized under solvothermal conditions.

Lately, various other COFs have been fabricated with the hydrazone linkage using the solvothermal technique, such as 


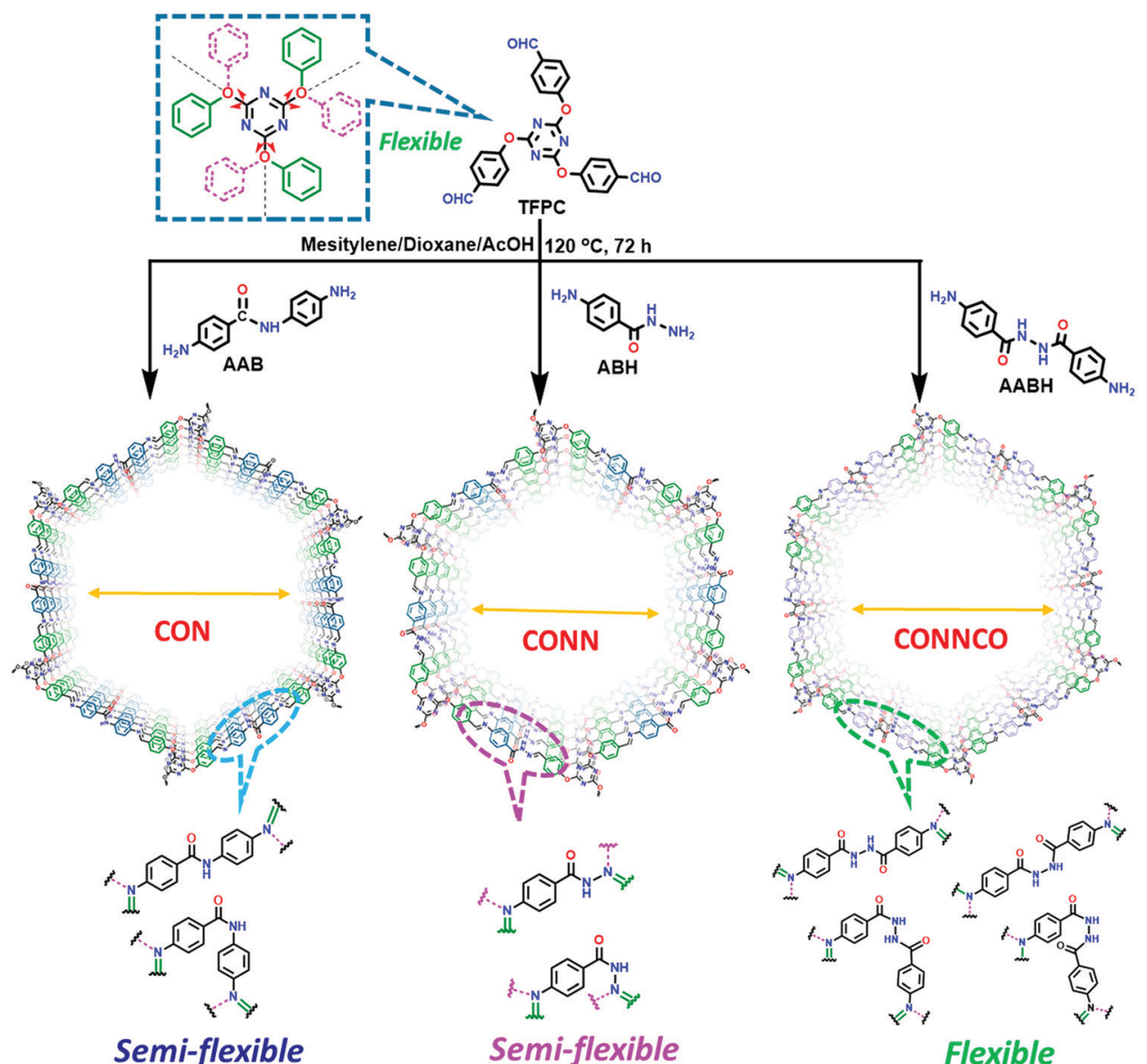

Fig. 10 Synthesis of CON, CONN, and CONNCO from highly flexible and semi-flexible linkages. Dotted lines indicate various possible conformers due to flexibility and semi-flexibility of linkers. Reproduced with permission from ref. 124, Copyright the American Chemical Society (2021).

NUS-50, ${ }^{130}$ NUS-51, ${ }^{130}$ COF-DB, ${ }^{131}$ COF-DT, ${ }^{131}$ COF-F $x-H,{ }^{132}$ $p-\mathrm{COF}_{10}{ }^{133}$ TFPA-Mth COF ${ }^{134}$ and XB-COF ${ }^{135}$ all of which are made from ditopic aromatic hydrazine and tritopic aldehydic units. Although the synthesis of the two hydrazone-based COFs, NUS-50 and NUS-51 (NUS = National University of Singapore), involved the use of water as the solvent, the products were obtained in good yields and high purity, exemplifying the good use of water in place of toxic organic solvents. The incorporation of $\mathrm{Co}(\mathrm{II})$ in these COFs resulted in good Lewis acid catalytic activity. The stable and fluorescent active TFPA-Mth COF was prepared via the Schiff-base solvothermal condensation of tris-(4formylphenyl) amine (Tfpa) with a hydrazide-based chiral monomer (Mth) at $120{ }^{\circ} \mathrm{C}$ for $72 \mathrm{~h}$. The two above-mentioned COFs were proven to be potential candidates for metal ion sensing (vide infra). In addition, flexible dihydrazine units have been used together with a trialdehyde monomer to give TpODH and TFPODH COFs via the imine condensation of oxalyldihydrazide $(\mathrm{ODH})$ and 1,3,5-triformylphloroglucinol (Tp) or 1,3,5-triformylbenzene (TFB) at $120{ }^{\circ} \mathrm{C}$ for $72 \mathrm{~h}$, respectively. ${ }^{136}$

Alternatively, the combination of a ditopic aromatic hydrazine with a tetratopic pyrene-based aldehydic unit under solvothermal conditions afforded TFPPy-DETHz COF in 2018 and Pythz-COF in 2021. The TFPPy-DETHz COF was obtained in
$86 \%$ via the condensation reaction of 1,3,6,8-tetrakis(4-formylphenyl)pyrene (TFPPy) and 2,5-diethoxyterephthalohydrazide (DETH) in a mixture of mesitylene and dioxane with acetic acid at $120{ }^{\circ} \mathrm{C}$ for $72 \mathrm{~h} .{ }^{137}$ Usually COFs having a hydrazone linkages display poor luminescence behavior (similar to an imine linkage) given that the $\mathrm{N}$-atoms of the linkage cause fluorescence quenching. However, scissoring the $\mathrm{N}-\mathrm{H}$ bond in the $\mathrm{N}$-site of the hydrazone moiety generates an $\mathrm{N}^{-}$anion, which shows improved light-emitting activity. Another pyrene-based COF, Pythz-COF, possesses high surface area and good crystallinity and exhibits excellent water sensing and adsorption behavior. ${ }^{138}$ To synthesize Pythz-COF, a Pyrex tube charged with 1,3,6, 8-tetrakis( $p$-formylphenyl)pyrene (Py) and terephthalic dihydrazide in a mixture of mesitylene/dioxane $(2: 1, \mathrm{v} / \mathrm{v})$ with acetic acid as a catalyst was sonicated for $10 \mathrm{~min}$, degassed, and then sealed under vacuum and heated at $120{ }^{\circ} \mathrm{C}$ for $5 \mathrm{~d}$.

In 2019, the mixed linkers amine-co-COF-OH and amine-coCOF-H were tailored with different amounts of 2,5-bis(2-(dimethylamino)ethoxy)-terephthalohydrazide (DtATH) with DETH and trialdehydes TFG/TFB, respectively. ${ }^{139}$ The synthesis was conducted under solvothermal conditions at $120{ }^{\circ} \mathrm{C}$ for $72 \mathrm{~h}$, yielding aminerich frameworks for $\mathrm{CO}_{2}$ sorption studies. It was found that with an increase in the content of the amine-functionalized monomer 
a
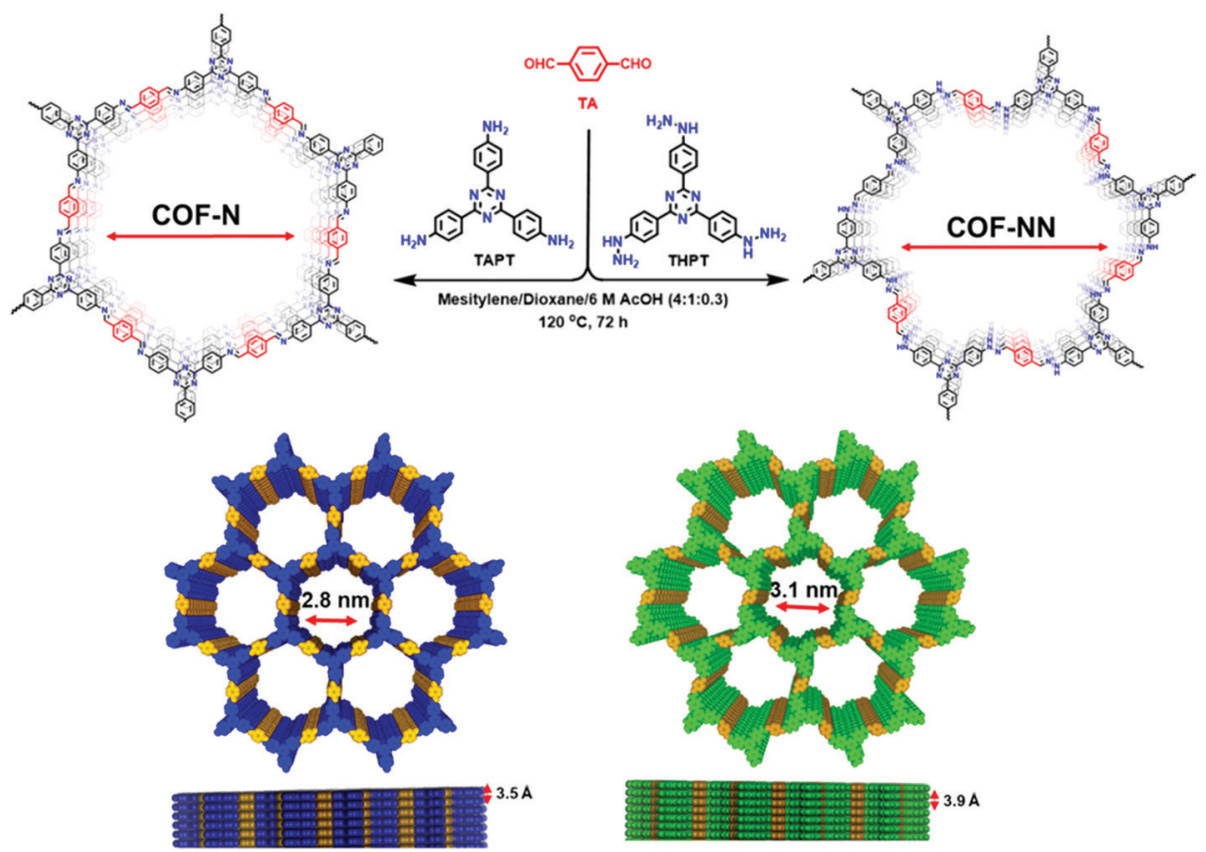

b

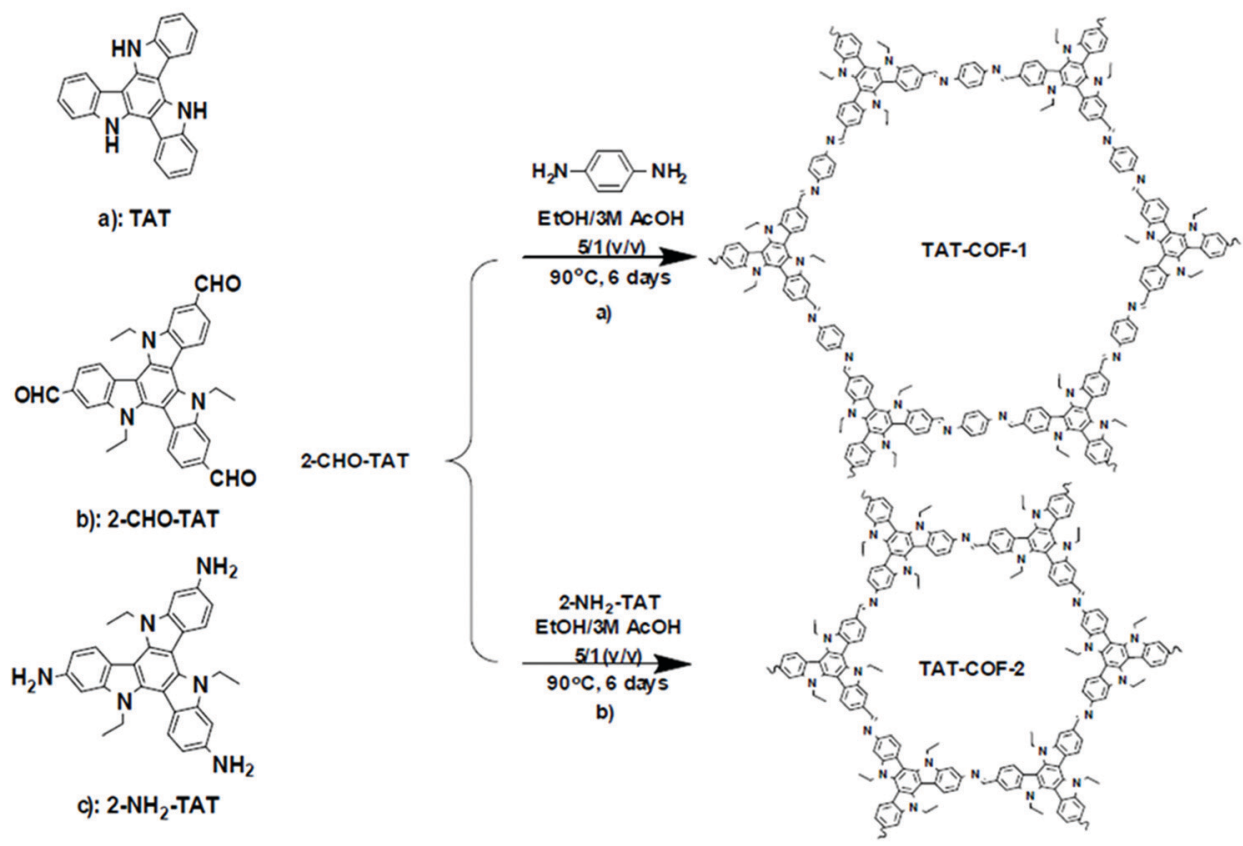

Fig. 11 (a) Synthesis of 2D imine-linked COF-N and COF-NN. (b) Structures of TAT model compound and its aldehyde and amine derivatives and synthesis of TAT-COF-1 and TAT-COF-2. Reproduced and adapted with permission from: (a) ref. 125, Copyright the American Chemical Society (2020) and (b) ref. 126, Copyright The Royal Society of Chemistry (2015).

DtATH, the BET surface area of both COFs was reduced by more than $50 \%$, leading to a decrease in their $\mathrm{CO}_{2}$ uptake capacity.

A few other hydrazone-linked COFs have been reported combining trihydrazine units with dialdehyde monomers, i.e., COF-ASB, Bth-Dha and Bth-Dma. The usual solvothermal condensation was employed for their synthesis, where the monomers of both the frameworks were heated at $120{ }^{\circ} \mathrm{C}$ for 3-5 days. The highly crystalline COF-ASB was prepared via the reaction between benzene-1,3,5-tricarbohydrazide (BTCH) and benzene1,4-dicarboxaldehyde (BDA) for $3 \mathrm{~d} .{ }^{140}$ The COF possesses high thermal and chemical stability. Its composite with $\mathrm{Ru}$ nanoparticles (Ru@COF-ASB) promotes the synthesis of imines from amines and alcohols in solvent-free aerobic conditions.

Two other thermally and chemically stable COFs, Bth-Dha and Bth-Dma, were synthesized via the reaction between benzene-1,3,5tricarbohydrazide (Bth) and 2,5-dihydroxyterephthalaldehyde (Dha) 
or 2,5-dimethoxyterephthaldehyde (Dma), respectively, for $5 \mathrm{~d}^{\mathbf{1 4 1}}$ Bth-Dma COF contains O,N, $\mathrm{O}^{\prime}$-chelating sites, which tend to bind $\mathrm{Fe}(\mathrm{III})$ ions selectively, acting as a potent sensor.

Mixed hydrazone-boroxine $\left(\mathrm{C}=\mathrm{N}-\mathrm{NH}_{2}, \mathrm{~B}-\mathrm{O}\right)$ linkage. A rare example in this category is the dual-pore COF-OEt, which was solvothermally fabricated using 3,5-diformylphenylboronic acid (DFPBA) and 2,5- diethoxyterephthalohydrazide (DETH) monomers. ${ }^{66}$ At elevated temperature, the boronic acid condensed to form a boroxine ring with the simultaneous formation of the hydrazone linkage. The framework was then subjected to hydrolysis to synthesize nanotubes with free $-\mathrm{OH}$ groups.

Aminal $\left(-\mathbf{C}\left(\mathbf{N R}_{\mathbf{2}}\right)\left(\mathbf{N R}_{\mathbf{2}}\right)\right.$ - linkage) $(\mathbf{R}=\mathbf{H}$, Alkyl). Very few examples have been reported in the literature with an aminal linkage. The design and fabrication of these crystalline materials is still challenging due to the extreme changes in the stereochemistry of their structure during the condensation reaction. The first aminal COF was reported in 2011, namely PAF-6, which was prepared from cyanuric chloride and piprazine. ${ }^{142}$ Recently, COFs, namely AMINAL COF-1 and AMINAL COF-2, were synthesized via the solvothermal condensation of piperazine and tetraaldehydes $\mathrm{A} 1$ and $\mathrm{A} 2$ with $D_{2 \mathrm{~h}}$-symmetry, respectively, at $120{ }^{\circ} \mathrm{C}$ for 3 days in sealed tubes. ${ }^{38}$

$\boldsymbol{\beta}$-Ketoenamine $\left(-\mathbf{C}=\mathbf{O},-\mathbf{N H}_{2}\right)$ linkage. The COF chemistry that combine reversible and irreversible reactions involves the formation of a $\beta$-ketoenamine linkage. Solvothermal conditions were employed with the monomers 1,3,5- triformylphloroglucinol (Tp) and $p$-phenylenediamine (Pa-1) or 2,5-dimethyl-p-phenylenediamine (Pa-2) to yield TpPa-1 and TpPa-2 COF powders, respectively. ${ }^{67} \mathrm{~A}$ well-sonicated concoction of monomers in dioxane/mesitylene was added to a Pyrex tube and subjected to prolonged heating at $120{ }^{\circ} \mathrm{C}$ for $72 \mathrm{~h}$. The detailed chemistry and stability aspects of TpPa-1 are mentioned in Section 3.1.
Similarly, the 2D DAAQ-TFP COF was tailored using 2,6-diaminoanthraquinone (DAAQ) and 1,3,5-triformylphloroglucinol (TFP) monomers in two different solvents, i.e., 1,4-dioxane and $N, N$ dimethylacetamide. ${ }^{143}$ The reaction temperature of the glass ampoule containing the reaction mixtures varied with the choice of solvent, i.e., dioxane-solvated mixture was heated at $120{ }^{\circ} \mathrm{C}$, yielding $\sim 60-70 \%$ product, while that with DMA was carried out at $90{ }^{\circ} \mathrm{C}$ for 2 days, yielding $\sim 70-85 \%$ product.

In 2016, the highly luminescent and semi-flexible COF-JLU4 was reported from the solvothermal condensation of 2,5dimethoxyterephthalohydrazide with triformylphloroglucinol (Fig. 12). ${ }^{144}$ The COF initially forms hydrazone bonding with further bonding in the $\beta$-ketoenamine linkage due to the hydroxyl-substituted trialdehyde group. The N-rich framework possessed exceptional chemical and moisture stability and was the first to be utilized as an efficient pH sensor (Section 6.2.2).

Likewise, many $\beta$-ketoenamine-linked frameworks have been constructed using high temperature-autogenous pressure conditions, such as, TAPB-TFP COF, iPr-TAPB-TFP COF ${ }^{145}$ nitrosubstituted $\operatorname{TpBD}\left(\mathrm{NO}_{2}\right) \mathrm{COF},{ }^{146}$ highly unsaturated TP-EDDA and TP-BDDA COFs, ${ }^{147}$ and methyl substituted 3D-TpBD-Me ${ }_{2}$ COF. $^{148}$ The synthesis of these COFs involves the use of a solvent combination of 1,4-dioxane/mesitylene with a catalytic amount of $\mathrm{AcOH}$ in the temperature range of $\sim 110-120{ }^{\circ} \mathrm{C}$ for $72-96 \mathrm{~h}$.

As shown in Fig. 13, Das et al. reported the construction of two COFs, TpBDH and TfpBDH, via the solvothermal reaction of 1,3,5-triformylphloroglucinol (Tp) and 1,3,5-tris(4-formylphenyl)benzene (Tfp), respectively, with pyromellitic- $N, N^{\prime}$-bisaminoimide (BDH). ${ }^{149}$ These COFs show moderate chemosensor ability due to their $\pi$-stacked 2D layers, which leads to poor electron mobility and unavailability of space for the guest analytes to interact. Thus, nanosheets of TpBDH and TfpBDH
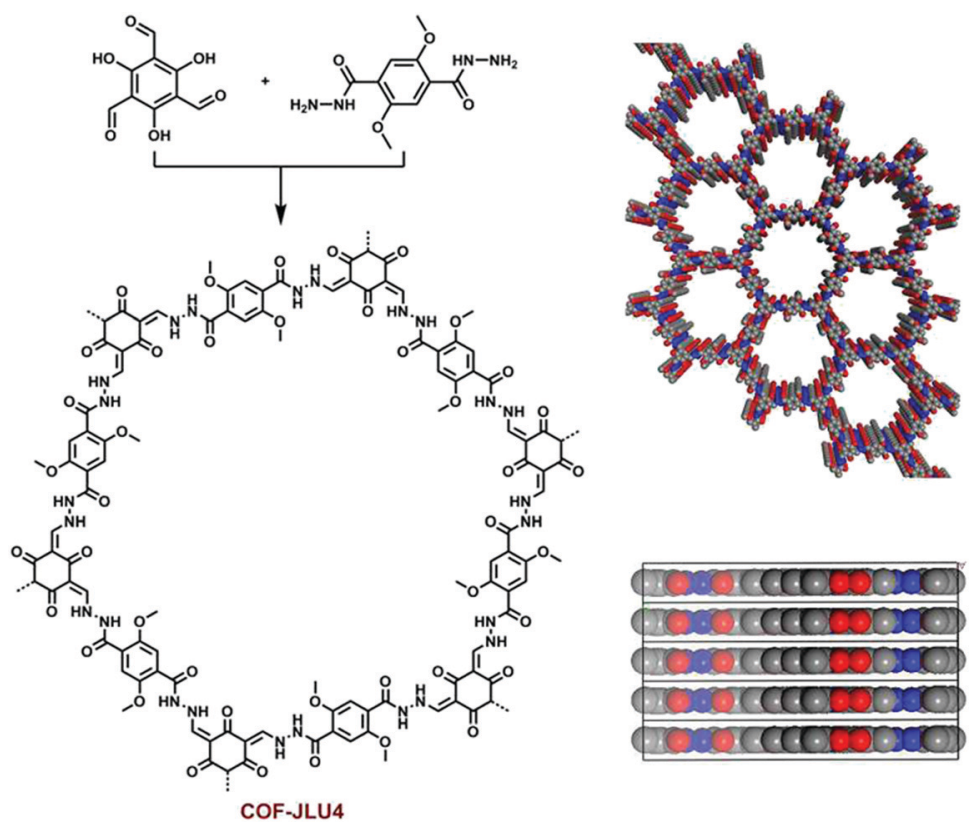

Fig. 12 Synthesis of $\beta$-ketoenamine-based COF-JLU4 and its structural packing. Reproduced with permission from ref. 144, Copyright The Royal Society of Chemistry (2016). 
a

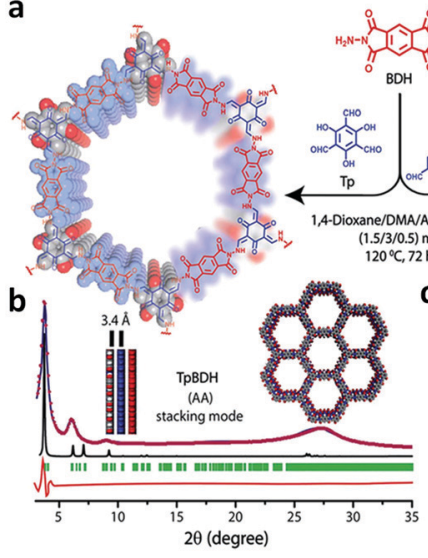

d

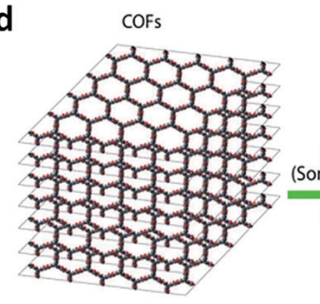

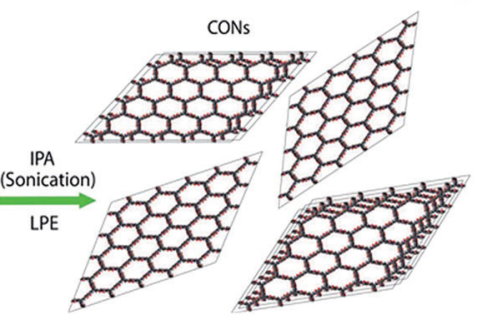

Fig. 13 (a) Schematic representation of the synthesis of $\mathrm{TpBDH}$ and $\mathrm{TfpBDH}$ [one unit of the space filling model of TpBDH and TfpBDH is shown in the inset of the chemical drawing modell. (b and c) Experimental PXRD patterns (blue) compared with simulated pattern (eclipsed; black) and Pawley refined difference between experimental and calculated data (red) [inset models show the eclipsed $A A$ and staggered $A B C$ stacking of consecutive 2D layers of TpBDH and TfpBDH, respectively]. (d) Schematic representation of COFs to CONs formation. Reproduced with permission from ref. 149, Copyright The Royal Society of Chemistry (2015).

CONs were obtained by exfoliating the respective bulk powders in the liquid phase using isopropyl alcohol at room temperature. AFM analysis confirmed the thickness of the nanosheets of $\sim 1.5-5.1 \mathrm{~nm}$ with $\sim 5-15$ layers stacked together (see Section 5.4.3). Consequently, the BET surface areas of TpBDH and TfpBDH-COFs to CONs were reduced from 642 and 738 to 270 and $112 \mathrm{~m}^{2} \mathrm{~g}^{-1}$, respectively. The two CONs exhibit a rare turn-on explosive sensing capability in comparison to their bulk materials (see Section 6.2.1).

The rapid, solvent-free, room temperature synthesis of TpPa1 (MC), TpPa-2 (MC) and TpBD (MC) COFs was performed via the mechanochemical (MC) grinding method. The monomers 1,3,5-triformylphloroglucinol (Tp) and $p$-phenylenediamine (Pa-1), 2,5-dimethyl-p-phenylenediamine (Pa-2), or benzidine (BD) were placed in a mortar and finely ground with a pestle for 40-45 min. ${ }^{83}$ A visible color change was observed at the time intervals of 5,15 and $40 \mathrm{~min}$, showing light-yellow, bright orange and dark-red colors, indicating extended $\pi$-conjugation, respectively (Section 6.1.1).

A hydrazone-linked TpTh COF underwent $\beta$-ketoenamine formation by using 1,3,5-triformylphloroglucinol (Tp) and terephthalic dihydrazide (Th) via enol-to-keto tautomerism under LAG conditions. ${ }^{94}$ The monomers, solvent mixture (1,4-dioxane and mesitylene) and $3 \mathrm{M} \mathrm{AcOH}$ catalyst were placed in a stainless steel jar with a $7 \mathrm{~mm}$ steel ball and milled for $90 \mathrm{~min}$. A yellow

powder with high crystallinity and stability was obtained in $\sim 78 \%$ yield.

A distinct method for the construction of highly crystalline micro and mesoporous COFs was performed using the Michael addition-elimination reaction in the aqueous phase under ambient conditions. The JUC-52x (JUC = Jilin University China, $x=0-3$ ) COF series with a pore size in the range of 1.59-2.92 nm was constructed. ${ }^{150}$ In a typical synthesis, a $C_{3}$-symmetric $1,3,5$ tris(3-dimethylamino-1-oxoprop-2-en-yl)benzene (TDOEB) unit was combined with four different amines, two of which are $C_{3}$-symmetric 1,3,5-tris(4-aminophenyl)triazine (TAPT) and 1,3,5tricarboxylic acid-tris(4-amino-phenyl-amide)benzene (TCTAB) and the other two are $C_{2}$-symmetric linkers, 2,5-dimethyl-1,4benzenediamine (DMB) and 3,30-dimethoxybenzidine (DMOB). The by-product of the reaction is dimethylamine, which further reacts with acetic acid used in the reaction to give dimethylamine acetate and dimethylacetamide, benefitting the formation of COFs with high crystallinity, porosity at high reaction rate (30 min), good yields $(\sim 93 \%)$ and scalable capability (up to $5.0 \mathrm{~g})$. Furthermore, the Fe(II)-loaded JUC-521 was efficiently exploited for the degradation of toxic pollutants in aqueous medium using the Fenton reaction.

Mixed imine- $\beta$-ketoenamine $(\mathbf{C}=\mathbf{N}, \mathbf{C}=\mathbf{0})$ linkage. The synthesis of COFs with mixed imine- $\beta$-ketoenamine linkages requires solvothermal conditions to form reversible Schiff bases and irreversible tautomerism, giving a $\beta$-ketoenamine linkage. Last year, three different COFs, namely NTU-BDA-HTA, NTU-BDA-DHTA, and NTU-BDA-THTA, were formed, which differ from each other in the linkage ratio due to different number of hydroxyl groups in the trialdehyde monomer unit. ${ }^{65}$ The first two COFs, as their name suggest, use mono- and di-hydroxy benzene-1,3,5-tricarbaldehyde (HTA and DHTA), which form an imine to $\beta$-ketoenamine ratio of $4: 2$ and $2: 4$, respectively. In contrast, the third COF, viz. NTU-BDA-DHTA, uses tri-hydroxy benzene-1,3,5-tricarbaldehyde (THTA) with benzene-1,4-diamine (BDA) having only $\beta$-ketoenamine linkages (linkage ratio $=0: 6$ ). Facilitating the framework with more stable $\beta$-ketoenamine linkages enhances the COF stability and makes the framework efficient for photocatalytic activity.

Borazine (B-N) linkage. The use of borazine as a precursor for the construction of porous materials is rare given that their synthesis is challenging. Compounds containing a B-N bond, resembling boron nitride $(\mathrm{BN})$ are generally accessible only at high temperature and pressure conditions. The first borazine-linked crystalline COF, BLP-2(H) ${ }^{60}$ was synthesized via the thermal decomposition of 1,3,5(p-aminophenyl)-benzene-borane (APBB) in a mixture of mesitylene/ toluene in a sealed tube at $120{ }^{\circ} \mathrm{C} / 150$ mTorr for $72 \mathrm{~h}$.

Phenazine linkage. CS-COF is a conjugated microporous polymer, which was efficiently synthesized under solvothermal conditions. ${ }^{151}$ In a Pyrex tube charged with triphenylene hexamine (TPHA) chloride and tert-butyl-pyrenetetraone (PT), a mixture of ethylene glycol and aq. AcOH acid was added and sonicated for $15 \mathrm{~min}$ at room temperature. The tube was then placed in an autoclave at $120{ }^{\circ} \mathrm{C}$ for $72 \mathrm{~h}$ to allow the ring fusion reaction, forming phenazine-linkages and the product was isolated in an appreciable amount ( 86-90\%). 


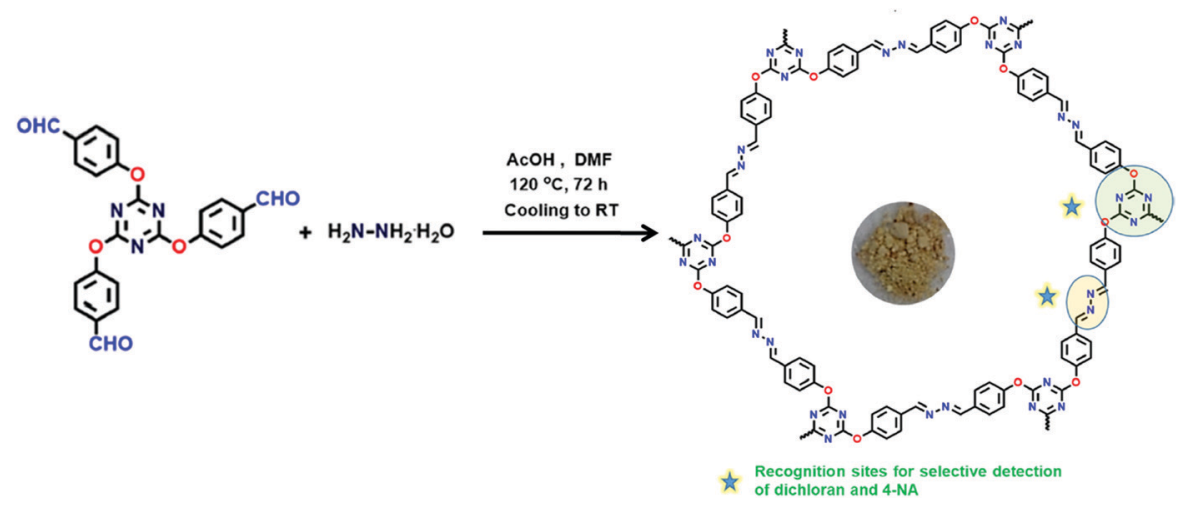

Fig. 14 Synthesis of azine-linked ANCOF, where the star marks represent the recognition sites for the selective detection of DCNA and 4-NA. Reproduced with permission from ref. 154, Copyright the American Chemical Society (2020).

Azine linkage. The first COF with an azine-linkage was synthesized in 2013. The Py-azine COF was obtained from the prolonged solvothermal condensation of hydrazine with $1,3,6,8^{-}$ tetrakis(4-formylphenyl)pyrene (TFPPy) in a solvent mixture of $o$-DCB $/ n$-BuOH with AcOH catalyst at $120{ }^{\circ} \mathrm{C}$ for $7 \mathrm{~d} .{ }^{152}$ Another 2D azine-linked ACOF-1 was prepared under similar conditions via the condensation of hydrazine hydrate and 1,3,5-triformylbenzene for $3 \mathrm{~d}^{153}$

Recently, using the solvothermal approach, a highly luminescent azine-linked framework, namely ANCOF, was constructed from tri(4-formylphenoxy)cyanurate (TFPC) and hydrazine monohydrate (Fig. 14) ${ }^{154}$ The framework is rich in nitrogen and with a high $\pi$-electron density to detect some electron-deficient species (vide infra).

Squarine linkage. A CuP-SQ COF was synthesized via the solvothermal condensation reaction of squaric acid (SA) and copper(II) 5,10,15,20-tetrakis(4-aminophenyl)porphyrin (TAP-CuP) in the presence of a mixture $o$-dichlorobenzene $/ n$-butanol at $85{ }^{\circ} \mathrm{C}$ for $7 \mathrm{~d}^{155}$ The resulting framework possesses planar squarine units, forming a zigzagged $\mathrm{N}$-rich conjugated $\mathrm{COF}$ skeleton.
Azodioxy linkage. The formation of an azidoxy bond involves the dimerization of nitroso units, which is a reversible reaction. Therefore, azidoxy-linked COFs require nitroso-functionalized monomeric units. Three of these frameworks have been reported, namely NPN-1, NPN-2 and NPN-3, which gave beautiful single-crystals (see Sections 3.2.2 and 5.4). ${ }^{88}$ For their synthesis, a solution of either tetrakis[4-(hydroxylamino)phenyl]methane, silane or adamantane was mixed with $\mathrm{Ag}_{2} \mathrm{CO}_{3} /$ Celite in a solvent mixture of benzene or mesitylene/ethanol, mesitylene/methanol and mesitylene/ethanol/THF and stirred for 5 min under ambient conditions and left undisturbed for $48 \mathrm{~h}$ to 1 week. Also, the room temperature synthesis of azidoxy-linked POR-COF was reported using the highly conductive N-rich porphyrin core (Fig. 15$).{ }^{35}$ The hydroxylamine-substituted protected porphyrin monomer was converted into the nitriso derivative in situ in the presence of tetrabutylammonium fluoride (TBAF) and THF solvent, followed by polymerization to give the desired COF.

Imide linkage. The polyimide (PI) COF series, PI-COF- $x$ $(x=1-5)$, was constructed in 2014 and 2015 via an imidization reaction under solvothermal conditions. PI-COF-1, PI-COF-2 and PI-COF-3 are 2D frameworks constructed using pyromellitic

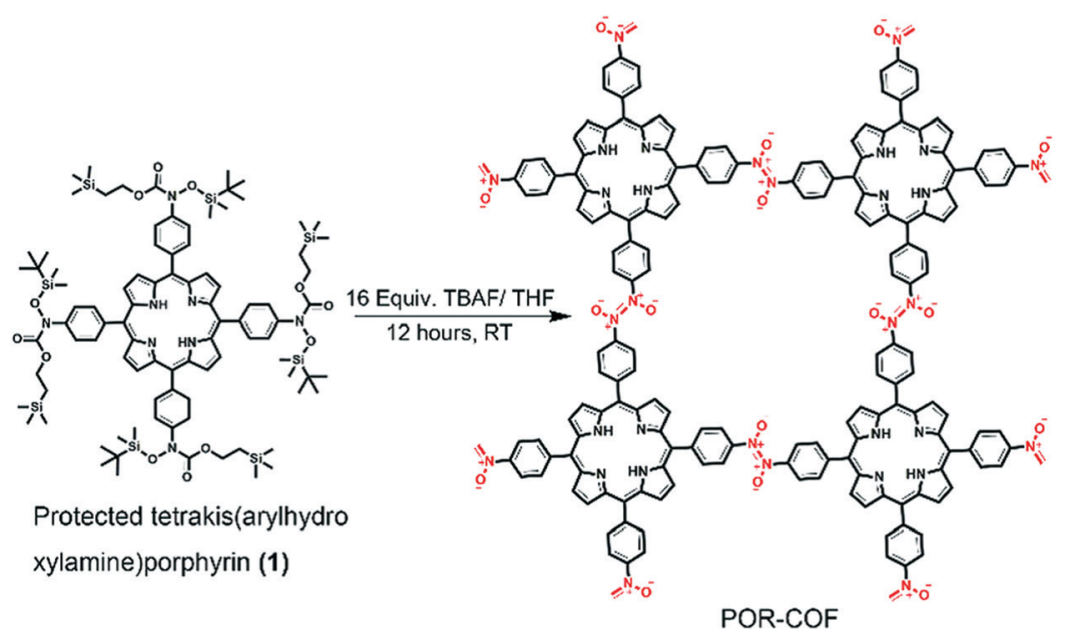

Fig. 15 Synthesis of azodioxy-linked porphyrin-based covalent organic framework (POR-COF). Reproduced with permission from ref. 35, Copyright The Royal Society of Chemistry (2016). 
dianhydride (PMDA) with tris(4-aminophenyl)amine (TAPA, $7 \AA$ ) , 1,3,5-tris(4-aminophenyl)benzene (TAPB, $9 \AA$ ) or 1,3,5tris[4-amino(1,1-biphenyl-4-yl)]benzene (ТАBPB, $13 \AA$ Å) in a mixture of N-methyl-2-pyrrolidone (NMP), mesitylene and isoquinoline, respectively. ${ }^{156}$ The reaction mixture was heated at $200-250{ }^{\circ} \mathrm{C}$ for 5-7 d, yielding the hcb net from the $\left[C_{3}+C_{2}\right]$ combination. Conversely, the 3D porous frameworks PI-COF-4 and PI-COF-5 were synthesized using pyromellitic dianhydride (PMDA) and a tetrahedral building block 1,3,5,7-tetraaminoadamantane (TAA) or tetra(4-aminophenyl)methane (TAPM), respectively, at $160{ }^{\circ} \mathrm{C}$ for $5 \mathrm{~d}{ }^{44}$ Surprisingly, the former 3D framework with an adamantane core was free from interpenetration possibly due to the steric bulk around the short imide-linked arms, whereas PI-COF-5 was weaved in a four-fold interpenetrated dia net.

Spiroborate linkage. Ionic covalent organic frameworks (ICOFs) with boron as the anionic center was prepared through the formation of a spiroborate linkage. ${ }^{37,157}$ These ICOFs, namely ICOF-1 and ICOF-2, contain a permanent immobilized ionic center, which allows the transportation of $\mathrm{DMA}^{+}$and $\mathrm{Li}^{+}$ ions. An inert $\mathrm{N}_{2}$ environment was maintained for their synthesis, where an octahydroxy-substituted macrocycle and trimethoxy borane in DMF were heated at $120{ }^{\circ} \mathrm{C}$ for 7 d. ICOF-1 utilizes dimethylamine, whereas ICOF-2 uses LiOH as the cationic source.

Olefinic $(-\mathrm{C}=\mathrm{C}-)$ linkage. An olefin-linked 2D conjugated COF, namely 2DPPV, was synthesized by the Knoevenagel polycondensation reaction of 1,3,5-tris(4-formylphenyl)benzene (TFPB) and 1,4-phenylene diacetonitrile (DCB) in a sealed Pyrex tube containing $\mathrm{Cs}_{2} \mathrm{CO}_{3}$ and $o$-dichlorobenzene and heated at $150{ }^{\circ} \mathrm{C}$ for $72 \mathrm{~h} .{ }^{158}$ Recently, two triazine-cored unsubstituted olefinic-linked COFs, g- $\mathrm{C}_{18} \mathrm{~N}_{3}$ - $\mathrm{COF}$ and g- $\mathrm{C}_{33} \mathrm{~N}_{3}$-COF, were synthesized via the solvothermal reaction of 2,4,6-trimethyl1,3,5-triazine (TMTA) with a $C_{2}$-symmetric linear aldehyde monomer, 1,4-diformylbenzene (DFB), and $C_{3}$-symmetric trigonal aldehyde precursor, 1,3,5-tris(4-formylphenyl)-benzene (TFPB), respectively, at $120{ }^{\circ} \mathrm{C}$ for $2 \mathrm{~h}^{159}$ The solvent combination of $o$ dichlorobenzene/ $n$-butanol was employed for both frameworks with $\mathrm{KOH}$ and EtONa reagents in the respective mixtures. A very common honeycomb topology is knitted for both the COFs. Similarly, solvothermal conditions were used to construct olefinicbonded COF- $701^{160}$ via the aldol condensation of 2,4,6-trimethyl1,3,5-triazine (TMT) and 4,4'-biphenyldicarbaldehyde (BPDA) and g- $\mathrm{C}_{34} \mathrm{~N}_{6}-\mathrm{COF}^{161}$ via the Knoevenagel condensation of 3,5-dicyano2,4,6-trimethylpyridine and 1,3,5-tris-(4-formyl-phenyl)triazine.

Benzimidazole linkage. The first semi-crystalline benzimidazole-based TpDAB COF was synthesized via the solvothermal Schiff base condensation of 1,3,5-triformylphloroglucinol (Tp) and $3,3^{\prime}$-diaminobenzidine (DAB) ${ }^{162}$ The extended $\pi$-conjugation system is responsible for its electronic and ionic conductivity, which facilitate the movement of ions within the micropores of the porous framework and make it a promising candidate for energy storage. Benzimidazole-linked polymers (BILPs) have attracted increasing interest in the scientific community due to the easy formation of the imidazole ring unit via the condensation of an amine and an aldehyde. A highly crystalline and fully conjugated COF, hcc-COF, was reported in 2019, using a light-promoted synthesis method. ${ }^{163}$ The monomers 1,2,4,5-tetraminobenzene tetrahydrochloride (TAB) and hexaketocyclohexane octahydrate were sealed in a quartz vial together with mesitylene/methanol solvents and purged with Ar followed by the addition of distilled water and $\mathrm{AcOH}$ catalyst. The mixture was sonicated for different for different intervals and irradiated with sunlight at wavelengths in the range of $200-2500 \mathrm{~nm}$ to produce the bulk powder of hccCOF. The same reactants were sonicated in NMP/mesitylene/ chloroform with $\mathrm{AcOH}$ catalyst and irradiated with solar light for $1 \mathrm{~h}$ to yield $3.8 \mathrm{~nm}$-thick hcc-COF thin films. Therefore, a new method was used to synthesize the benzimidazole-linked framework in the bulk and thin film forms, which were significantly characterized using various techniques (see Section 5). Another triazine-based benz-bis(imidazole)-linked COF, namely TBICOF, was synthesized via the solvothermal polycondensation reaction of tri(4-formylphenoxy)cyanurate (TFPC), 1,2,4,5-tetraminobenzene tetrahydrochloride (TAB) and imidazole in a mixture of mesitylene/ N-methyl-2-pyrrolidone/dioxane at $120{ }^{\circ} \mathrm{C}$ for $72 \mathrm{~h}^{68}$ The benzimidazole-connected frameworks are rich in nitrogen and possess extraordinary thermal and chemical stabilities due to the irreversible bond locking by cyclization (see Section 3.1).

Benzo-bis(oxazole) linkage. This linkage is another example of an exceptionally stable linkage built over both reversible and irreversible reaction pathways (see Section 3.1). Two of these COFs, namely BBO-COF 1 and BBO-COF 2, were obtained via the reaction of 1,3,5-triformylbenzene (TFB) and 1,3,5-tris(4formylphenyl)benzene (TFPB), respectively, with 2,5-diamino1,4-benzenediol dihydrochloride (DABD) in two steps as follows: (1) the monomers were stirred in DMF at $-15{ }^{\circ} \mathrm{C}$ for $3 \mathrm{~h}$ for the formation of a phenolic imine-linked intermediate and (2) the addition of methanolic solution of $\mathrm{NaCN}$ and heating at $130{ }^{\circ} \mathrm{C}$ for $4 \mathrm{~d}$ in an aerobic environment. ${ }^{69}$ The framework exhibited a high surface area, and exceptionally high thermal, chemical and water stability. Recently, a triazine-cored benzo-bis(oxazole)linked TPT-DAHQ COF was constructed via the one-pot [3+2] solvothermal condensation of 2,4,6-tris(4-formylphenyl)triazine (TPT-3CHO) and 2,5-diaminohydroquinone dihydrochloride (DAHQ.2HCl) in the presence of DMF at $120{ }^{\circ} \mathrm{C}$ for 3 days. ${ }^{164}$ The time-dependent study showed the growth of ribbon-like crystallites into microtubes at intervals of 12, 24 and $48 \mathrm{~h}$ (see Section 5.4). The framework is rich in nitrogen atoms, which makes it efficient for energy storage and sensing applications.

Amide $(\mathrm{HN}-\mathrm{C}=\mathbf{O})$ linkage. Two polyamide-based COFs, $\mathrm{CAF}-1$ and CAF-2, were synthesized under very harsh solvothermal conditions via the devitrification method (Section 3.2.2). ${ }^{86}$ The amorphous monomers, polyamide(1,3,5-tricarboxybenzene-nettrans-1,4-cyclohexanediamine) (PATnC) (for CAF-1) and poly-

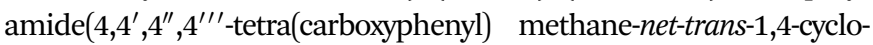
hexanediamine)(PATCnC) (for CAF-2), were dried under vacuum to remove the moisture content and kept at $250{ }^{\circ} \mathrm{C}$ in a pressure reactor for $3 \mathrm{~d}$ (CAF-1), and $240{ }^{\circ} \mathrm{C}$ in an oven for $7 \mathrm{~d}$ (CAF-2).

Silicate linkage. The one-pot self-assembly method was employed for the formation of silicate organic frameworks based on a hexacoordinated $\mathrm{SiO}_{6}{ }^{2-}$ moiety. Examples are the anionic SiCOF with alkali metal ions ( $\mathrm{M}=\mathrm{Li}, \mathrm{Na}$, and $\mathrm{K}$ ) named SiCOF $\mathrm{M}_{2}\left[\mathrm{Si}\left(\mathrm{C}_{16} \mathrm{H}_{10} \mathrm{O}_{4}\right)_{1.5}\right]{ }^{165}$ The monomers 9,10-dimethyl-2,3,6,7-tetrahydroxyanthracene (DMTHA) and tetramethyl orthosilicate (TMOS) 
in the presence of a suitable alkali-metal methoxide base were added to a Teflon-lined vessel and kept at $180{ }^{\circ} \mathrm{C}$ for $4 \mathrm{~d}$, yielding up to $97 \%$ product.

Viologen linkage. Viologen-based linkages contain charged 4,4'-bipyridinium moieties, which are widely used due to their rich redox chemistry. These linkages exhibit three redox states, which can be accessed electrochemically and also vary from hydrophilic to hydrophobic in nature. Due to this feature, these materials are promising candidates to adsorb multiple dyes with different polarities. The first viologen-based COF was reported in 2017 in the form of hollow spheres (HS) or hollow tubes (HT), which were prepared by the Zincke reaction between 1,1'-bis(2,4-dinitrophenyl)-[4,4'-bipyridine]-1,1'-diium dichloride (BDB) and 1,3,5-tris(4-aminophenyl)benzene (TAPB), in different solvents. ${ }^{166}$ The amorphous materials, HS and HT, were prepared in ethanol:water $(4: 1)$ and dioxane, respectively, by employing the solvothermal method for $3 \mathrm{~d}$ or using the microwave technique for $2 \mathrm{~h}$. In addition, a free-standing crystalline covalent organic gel framework (COGF) was made in a mixture of ethanol and water via microwave irradiation. The formation of hollow spheres and tubes was confirmed and observed using various microscopic characterization techniques (vide infra).

In another viologen-linked dicationic- $\mathrm{COF}, \mathrm{COP}^{++}$, the hexatopic cyclophosphazene core was combined with $\mathrm{CH}_{3} \mathrm{CN}$ under the microwave-assisted Menshutkin reaction. ${ }^{167}$ This framework was well exploited for the adsorption of dyes with varying polarities. The polarity of $\mathrm{COP}^{++}$was varied by reducing it from the dicationic form to the radical cationic form $\left(\mathrm{COP}^{\bullet+}\right)$, both of which are hydrophilic and match the polarities of fluorescein (neutral) and rhodamine $\mathrm{B}$ (cationic) dyes. However, $\mathrm{COP}^{++}$adsorbs fluorescein dye more selectively by up to $90 \%$. The neutral framework $\mathrm{COP}^{0}$ exhibits a hydrophobic nature and efficiently adsorbs Nile red up to $85 \%$.

Two porphyrin-based viologen-linked COFs, Zn-PV-COF and Red-PV-COF, were synthesized under microwave conditions, heating at $90{ }^{\circ} \mathrm{C}$ for 2 h. ${ }^{168}$ The Zincke reaction occurs between the amino-derivative of porphyrin and Zincke salt in an ethanol/ water solvent mixture to give PV-COF. This COF was further metallated with zinc ions in a methanol/chloroform mixture at $50{ }^{\circ} \mathrm{C}$ for $24 \mathrm{~h}$ to yield Zn-PV-COF. Conversely, neutral Red-PVCOF was obtained via the stirring of PV-COF at room temperature for $15 \mathrm{~min}$, where its bipyridinium units were chemically reduced using cobaltocene. The azo-bridged viologen- and calix[4]arenebased cationic covalent organic polymers (COPs) denoted as $\mathrm{COP}^{++}$and $\mathrm{COP} 2^{++}$, respectively, were prepared by the formation of a diazo linkage between a nitro derivative of calix[4]arene (pTNC4A) and two different viologen-containing diamines in the presence of a strong base in a Schlenk tube at $150{ }^{\circ} \mathrm{C}$ for $1 \mathrm{~d} .{ }^{169}$

Oxazole and thiazole linkage. Two new covalently bonded frameworks with oxazole and thiazole-connected units were constructed via the linker exchange method under solvothermal conditions. The imine-linked ILCOF-1 was employed as the precursor and added to 2,5-diaminobenzene-1,4-dithiol dihydrochloride (DAHQ·2HCl) or 2,5-diaminohydroquinone dihydrochloride (DAHD-2HCl) to give COF-921 and LZU-192, respectively. These two COFs required slightly different reaction conditions, i.e., for
COF-921: DMF/water in $75: 25$ at $85{ }^{\circ} \mathrm{C}$ for 1 day, and for LZU$192: 85: 15 \mathrm{DMF} /$ water at $85{ }^{\circ} \mathrm{C}$ for 2 days. $^{70}$ The frameworks are rich in $\mathrm{N}, \mathrm{O}$ and $\mathrm{S}$-heteroatoms, rigid and exhibit enhanced thermal, chemical stability under harsh acidic and basic conditions. Another thiazole-linked TTT COF was synthesized using post-synthetic locking of an imine-linked TTI-COF under very harsh thermal conditions. ${ }^{170}$ The activated TTI-COF and molten sulphur $\left(\mathrm{S}_{8}\right)$ were heated at $155{ }^{\circ} \mathrm{C}$ for $3 \mathrm{~h}$. The reaction mixture was well-mixed given that sulphur attains minimum viscosity at this temperature. Subsequently, the harsh thermal environment of $350{ }^{\circ} \mathrm{C}$ was provided for another $3 \mathrm{~h}$, and then the mixture was left to cool to room temperature. The extra sulphur was removed by Soxhlet extraction with a toluene/THF mixture for $24 \mathrm{~h}$. The imine bond locking by thiazole-ring formation enhanced the stability and robustness in the framework.

Urea-based linkage. The flexible urea-based linkage was established in COF-117 and COF-118 from the solvothermal imine-condensation of 1,3,5-triformylphloroglucinol (TFP) and 1,4-phenylenediurea (BDU) or 1,1'-(3,3'-dimethyl-[1,1'-biphenyl]$4,4^{\prime}$-diyl)diurea (DMBDU) in NMP/trichlorobenzene and $6 \mathrm{M}$ aq $\mathrm{AcOH}$ at $85{ }^{\circ} \mathrm{C}$ for $3 \mathrm{~d} .{ }^{81}$ The major challenge in the construction of these frameworks is the various conformations of the linkers due to their low $\mathrm{C}-\mathrm{N}$ rotational energies, which is easily overcome under high temperature reaction conditions (vide supra, Section 3.2.1).

This year, harsher conditions with prolonged time were employed for the synthesis of a urea-linked Pyurea COF. ${ }^{138}$ The monomers 1,4-phenylenediurea and 1,3,6, 8-tetrakis( $p$-formylphenyl)pyrene (Py) were taken in a Pyrex tube with NMP/AcOH (6M) in a ratio of 1:0.1 and sonicated for $10 \mathrm{~min}$, degassed, and then sealed under vacuum and heated at $120{ }^{\circ} \mathrm{C}$ for $5 \mathrm{~d}$.

Dioxin linkage. In 2018, two 2D COFs, COF-316 and COF-318, were synthesized under solvothermal conditions in which the triangular monomer 2,3,6,7,10,11-hexahydroxytriphenylene (HHTP) combines with either the linear tetrafluorophthalonitrile (TFPN) or 2,3,5,6-tetrafluoro-4-pyridinecarbonitrile (TFPC) in 1,4-dioxane/ triethylamine and dioxane/mesitylene/trimethyl-amine, respectively, at $120{ }^{\circ} \mathrm{C}$ for $3 \mathrm{~d}^{62}$ The fluorine atoms on the nitrile monomers are electron-withdrawing moieties, which tend to increase the extent of nucleophilic attack by the hydroxyl group of HHTP, resulting in a nucleophilic aromatic substitution $\left(S_{N} A r\right)$ reaction. An appropriate basic environment is required to carry out the reversible nucleophilic attack, which is followed by irreversible ring closure, directing the linkers toward a rigid and long-range ordered framework.

Later, the microwave-assisted nucleophilic substitution reaction was also employed to synthesize COF-318 (here, named TH-COF). ${ }^{96}$ Both monomers, HHTP and TFPC, were placed in an ampoule and sealed with a solvent mixture of dioxane/mesitylene/triethylamine and treated with microwave heating at $70{ }^{\circ} \mathrm{C}$ for $30 \mathrm{~min}$, giving $71 \%$ yield. Its surface area of $1254 \mathrm{~m}^{2} \mathrm{~g}^{-1}$ is much higher than that synthesized under solvothermal conditions $\left(576 \mathrm{~m}^{2} \mathrm{~g}^{-1}\right)$.

A series of dioxin-linked metallophthalocyanine COFs, MPcTFPN COF ( $\mathrm{M}=\mathrm{Ni}$, Co, and $\mathrm{Zn}$ ), was prepared using reticular chemistry. ${ }^{171}$ The $\mathrm{Et}_{3} \mathrm{~N}$-catalysed nucleophilic substitution reaction occurs between 2,3,9,10,16,17,23,24-octahydroxyphthalo -cyaninato metal (MPc-8OH; M = Ni, Co, and Zn) and tetrafluorophthalonitrile (TFPN) 
in DMA/mesitylene placed at $150{ }^{\circ} \mathrm{C}$ for $3 \mathrm{~d}$. The framework possesses high thermal stability $\left(\sim 400\right.$ in $\mathrm{N}_{2}$ and $\sim 300$ in $\mathrm{O}_{2}$ environment) and chemical stability (in common organic solvents and conc. acid/base conditions), which are attributed to the rigid metalophthalocyanine units.

Ester $(\mathbf{O}=\mathbf{C}-\mathbf{O}-)$ linkage. An ester is a rarely exploited linkage in COF chemistry. A solvothermal synthetic method was used to construct a series of ester-linked COFs, COF-119, COF-120,COF-121 and COF-122. ${ }^{63}$ Here, the trans-esterification reaction takes place between the hydroxyl-functionalised tri- or tetratopic units and ditopic 2-pyridinyl aromatic carboxylates. The reaction between di(pyridin-2-yl) terephthalate (DPT) and tetrakis(4-hydroxyphenyl)ethylene (THPE) or 1,3,5-tris(4-hydroxyphenyl)benzene (THPB) yields COF-119 with the kgm topology and COF-120 with hcb topology, respectively, in the presence of DBU/dioxane at $150{ }^{\circ} \mathrm{C}$ for $3 \mathrm{~d}$. Furthermore, the reticulated COF121 was obtained by reacting THPB with di(pyridin-2-yl) $\left[1,1^{\prime}\right.$ biphenyl]-4,4'-dicarboxylate (DPBP). To further explore the reticulation of COF-122, triol- and methyl-substituted dicarboxylates with extended arms were used under similar reaction conditions, yielding the hcb net with a high surface area.

Pyrimidazole linkage. The synthesis of the pyrimidazolebased linkage within a framework requires the solvothermal Groebke-Blackburn-Bienaymé (GBB) reaction based on isocyanide chemistry. A series of pyrimidazole-based COFs named LZU-56x ( $x=1$ to 8 ) and synthesized via the reaction between the derivatives of aminopyridine, 1,3,5-tris(3-fluoro-4-formylphenyl)benzene (TFFPB) and 1,3,5-tris(4-isocyanophenyl)benzene (TICB). ${ }^{172}$ The $p$-toluenesulfonic acid monohydrate $\left(\mathrm{PTSA} \cdot \mathrm{H}_{2} \mathrm{O}\right)$ catalyst was added to an ethanol/mesitylene solvent mixture (for LZU-561) in a glass tube, subjected to freeze-pump-thaw cycles, and then heated at $120{ }^{\circ} \mathrm{C}$ for $5 \mathrm{~d}$. The optimized solvent conditions with different substituents on the aminopyridine unit give high-order frameworks. By switching the tritopic isocyano unit to the ditopic diisocyanobenzene, LZU-564 to LZU-568 were fabricated with different solvent combinations.

Polycubane linkage. A rare polycubane-linked COF uses boron-phosphoric acid-based (BPA) linkages, which after subjecting to harsh solvothermal conditions formed cubes and rods. ${ }^{57}$ BP-COF-1 was synthesized by dissolving BPA-1 in a mixture of DMSO/toluene with methylphosphonic acid and sealing in a Pyrex tube, and then heating at $120{ }^{\circ} \mathrm{C}$ for $3 \mathrm{~d}$ (Fig. 16). It should be noted that the formation of the body-centered cubic (bcc) BP-COF-1 was unsuccessful with the polycondensation of diboronic and diphosphonic acid monomers. The thermal conditions for BP-COF-4 and BP-COF-5 were $100{ }^{\circ} \mathrm{C}$ for $3 \mathrm{~d}$, which use different linkers, BPA-4 and BPA-5. To synthesize BPCOF-6, a Pyrex tube was loaded with BPA-1, conc. $\mathrm{HCl}$, and $\mathrm{DMSO} /$ toluene and kept in a programmable oven at $120{ }^{\circ} \mathrm{C}$ for $5 \mathrm{~d}$. This is the first example of a COF with a very high content of connecting networks (see Section 2.2).

\subsection{Mechanistic studies for the synthesis of COFs}

In the aforementioned sections, it can be seen that there is a large number of linkages from which highly crystalline COFs are obtained when the monomers are employed under suitable

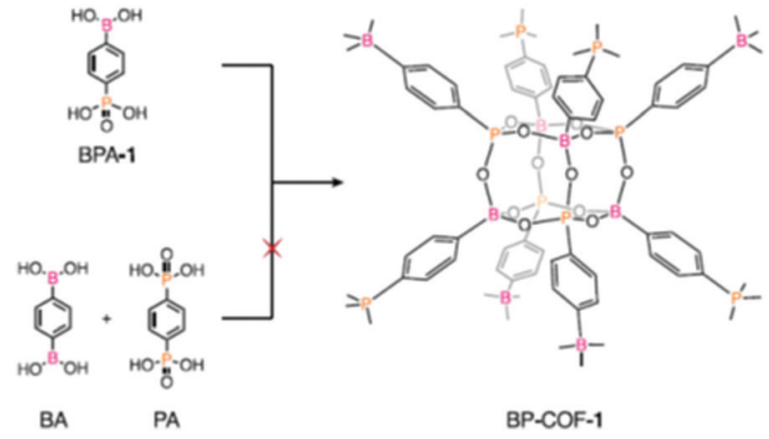

Fig. 16 Synthesis of BP-COF-1. Reproduced and adapted with permission from ref. 57, Copyright The American Association for the Advancement of Science (2020)

reaction methods and conditions. However, very few reports elaborate on the mechanistic pathways to describe how these monomers combine to give long-range orders and what factors affect the crystalline growth. In this section, we emphasize the mechanistic details of two widely explored linkages, i.e., boronic ester and imine, formed with different synthetic methods under different conditions.

4.3.1 COFs with boronic ester linkages. In-depth insight into the formation of COFs via boronic ester linkages was gained in the past five years by Dichtel and co-workers. It was observed for a long time that the formation of COFs is a heterogeneous process, where the monomers and organic polymer co-precipitate, yielding a framework with certain limitations, such as limited/low crystallinity, framework stability, and surface area. Thus, to overcome these shortcomings, the COF synthetic conditions were investigated by Smith et al. in 2014, where they reported a more efficient method for the synthesis of boronic ester-linked COF-5 under high temperature homogeneous conditions. ${ }^{5}$ It was concluded that by starting the reaction from a homogeneous mixture of HHTP and PBBA, COF-5 was more crystalline with a higher BET surface area $\left(\sim 2000 \mathrm{~m}^{2} \mathrm{~g}^{-1}\right)$ in comparison to that previously reported in the literature (see Table 3, entry 2). Homogeneity of the starting mixture was gained by adding the liquid additive $\mathrm{MeOH}$ ( $\sim 15$ equiv. of HHTP) and increasing the amount of dioxane in the solvent mixture. The turbidity experiments depicted that after heating the reaction mixture to $90{ }^{\circ} \mathrm{C}$ within a $2 \mathrm{~min}$ induction period, a precipitate begin to appear, yielding $75 \%$ COF product after $20 \mathrm{~h}$ reaction time. The additional kinetic studies indicate that the formation rate of COF-5 is second order with respect to [diol] and [boronic acid], but has an inverse second- and first-order dependence on the concentration of $\mathrm{MeOH}$ and TCAT, respectively. TCAT, as a uni-functional catechol-based modulator, competes with the diol monomer HHTP to slow down the polymer formation time; therefore, increasing the rate of COF formation.

The next year, another study was published by the same group focusing on the rate of framework growth and nucleation and factors inducing moisture stability in the product. ${ }^{173}$ It has been elucidated that the rate-determining step in the boronic ester-linked COF formation reactions is not the formation of the $\mathrm{C}_{2} \mathrm{O}_{2} \mathrm{~B}$ ring, namely, the $2 \mathrm{D}$ intermolecular stacking path 
occurs when growth of already existing micro-crystallite COFs predominates. Furthermore, factors such as controlled temperature $\left(60-90{ }^{\circ} \mathrm{C}\right.$ ), aromaticity of monomers (pyrene vs benzene-core), amount of competitor (TCAT) used in the beginning or during the reaction, and quantity of liquid additives ( $\mathrm{MeOH}$ and $\mathrm{H}_{2} \mathrm{O}$ ) are the deciding factors influencing the crystallinity and stability of the COF.

The effect of modulator on the microwave-assisted synthesis of COF-5 was closely studied by Bein and co-workers in 2016 using the functionalized truncated boronic acids, MPBA and CPBA. ${ }^{174}$ The reaction mixture was sealed in a microwave tube and heated to $100{ }^{\circ} \mathrm{C}$ at $300 \mathrm{~W}$ power. The report showed that with $10 \%$ modulation (HHTP: BDBA: modulator $=2: 2.70: 0.6$ ), the reaction time increased by $30 \mathrm{~min}$, forming a large number of crystallite domains with sizes of 60 and $56 \mathrm{~nm}$ for MPBA and CPBA, respectively. Moreover, the highest XRD intensity and BET surface area were obtained under these conditions, i.e., $2100 \mathrm{~m}^{2} \mathrm{~g}^{-1}$, which is $\sim 35 \%$ greater than that of the unmodulated COF-5. Further studies done by Dichtel's group revealed that the use of nitrile-containing co-solvents help in stabilizing the colloidal suspension for over a month. ${ }^{175}$ This promotes the growth of $2 \mathrm{D}$ COFs by inhibiting the particle aggregation, ameliorating the quality of its single crystals. Later, in a follow-up paper published in 2018 by the same group, it was proven that control of the crystallite size was achieved via the rate of monomer addition after stabilizing the COF colloidal suspension. ${ }^{176}$ The SAXS/ WAXS studies showed that with the slow addition of the monomer $\left(0.1\right.$ equiv. $\left.\mathrm{h}^{-1}\right)$, the crystallinity of the framework increased, suppressing the nucleation process, which contradicts the results of fast monomer addition (1.0 equiv. $\mathrm{h}^{-1}$ ). These studies provide a way to overcome the crystallite growth and nucleation issues for knitting boronic ester-linked COFs.

Based on the summary of the above-discussed information, a comprehensive mechanism for the formation of a COF with boronic ester linkages under either high temperature or microwave-assisted conditions is thoroughly displayed in Fig. 17A.

During the formation of COF-5 from HHTP and PDBA, the monomers initially combine to give oligomers in step $a$, which then stack reversibly in step $b$. During this stage, an addition of diol or boronic acid-mimicking modulators controls the growth by slackening the reaction. On proceeding further, either the oligomeric sheets stack together forming metastable layers, or one sheet acts a base for template polymerization. Consequently, each stage gives COF crystallites through step $c$, which require further treatments to get the desired product. Control of the various aforementioned factors in the early stages helps to build the crystallites at this stage. By recovering these crystallites, a multi-crystalline precipitate is obtained via step $d$, whereas treating them with suitable stabilizing agents yields single crystals by preventing nucleation (step $e$ ). This can occur when the monomers are slowly added at this point. On the contrary, the fast addition of the monomer yields new crystallite particles via nucleation, and thus single crystal growth is prevented.

4.3.2 COFs with imine linkages. After boronic ester bonds, the imine linkages are widely explored to weave a long list of single crystalline, water-stable COFs. The Schiff base imine
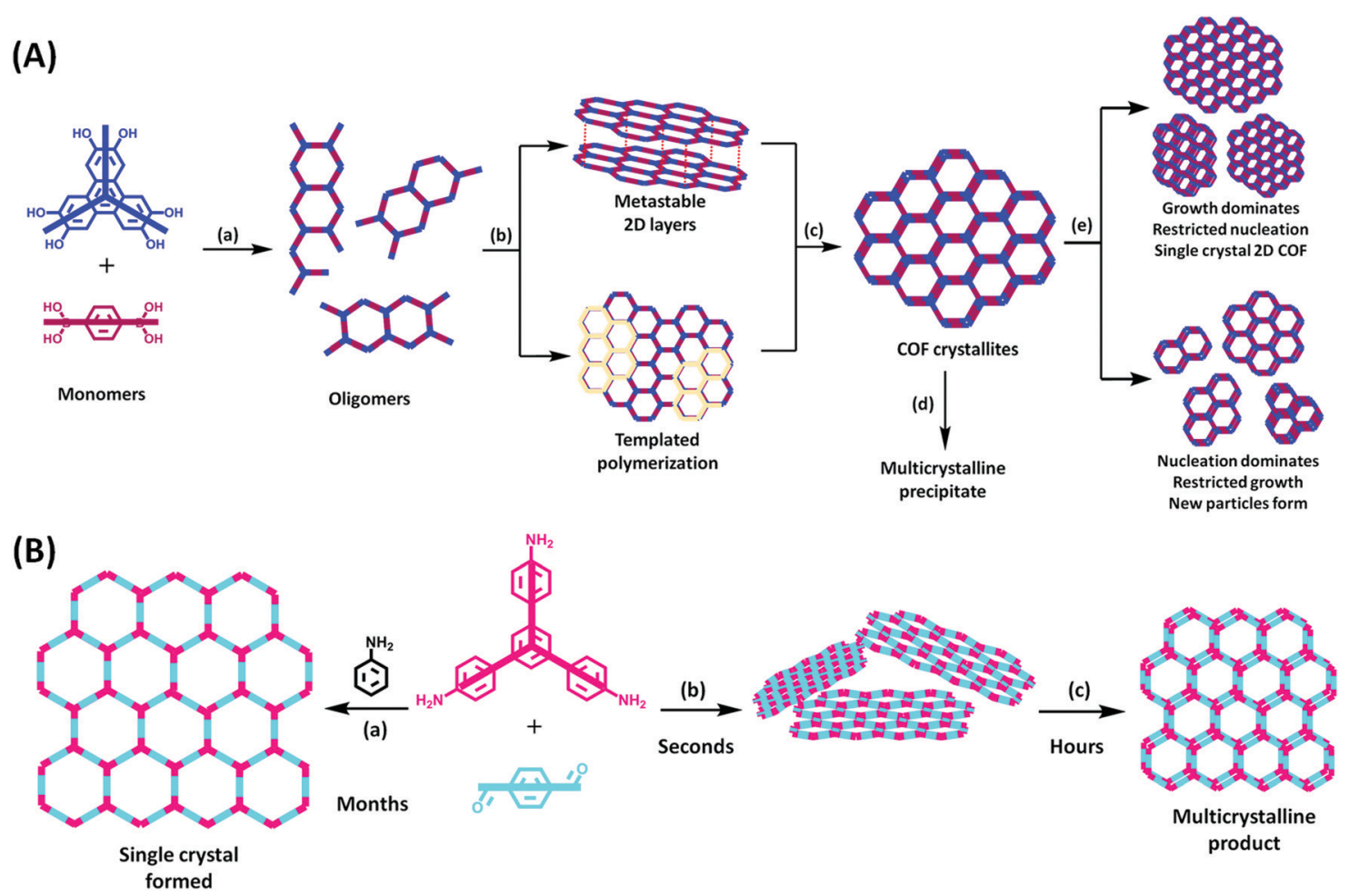

Fig. 17 Mechanism for the formation of (A) 2D boronic ester-linked and (B) imine-linked COFs proposed by analyzing the information from the detailed mechanistic studies. Adapted from ref. 5, 173-177, 180 and 182. 
condensation reactions are very fast, and therefore, pose difficulty in building crystalline 2D frameworks from amorphous organic polymers. Reports have shown that these linkages are extensively formed under solvothermal conditions, where thermodynamic equilibrium is achieved in a few days, yielding the desired uniformity. Similar but distinctive in-depth studies were performed by Smith et al. in 2016 to understand the mechanism of imine-linked 2D COFs, using TAPB and PDA as monomers in particular. ${ }^{177}$ Accordingly, a homogeneous solution of reactants with an optimized dioxane/mesitylene ratio $(4: 1 \mathrm{v} / \mathrm{v})$ was heated to $70{ }^{\circ} \mathrm{C}$ for 5 days. The glacial acetic acid catalyst and $\mathrm{H}_{2} \mathrm{O}$ content were maintained for the minimum quantity to be $\geq 1 \mathrm{M}$ and $3.3 \mathrm{M}$, respectively, which was required to initiate crystallinity within the framework and attain higher yields. Additionally, the BET surface area of the initial amorphous solid was found to be $18 \mathrm{~m}^{2} \mathrm{~g}^{-1}$, which further increased to $287 \mathrm{~m}^{2} \mathrm{~g}^{-1}$ over a period of two days. This supports the observation that the amorphous-to-crystalline imine COF transformation is a two-step process, i.e., fast polymerization of soluble monomers to an amorphous organic polymer at elevated temperature followed by the imine exchange process, which increases the tendency of error-correction.

A big step was taken by Yaghi and co-workers by judiciously adding a suitable amine-based modulator to obtain 3D iminelinked COFs. The widely used modulator is aniline, which is a monofunctional mimic of the diamine monomer and competes in the COF formation reaction. Consequently, large-size crystals of good quality grow by inhibiting nucleation. By following the aniline modulation process, large single crystals (size in parentheses) of COF-300 $(\sim 60 \mu \mathrm{m})$, COF-303 $(\sim 15 \mu \mathrm{m})$, LZU-79 $(\sim 100 \mu \mathrm{m})$ and LZU-111 $(\sim 50 \mu \mathrm{m})$ were obtained. ${ }^{6}$ In all cases, good-quality crystals were obtained under solvothermal conditions when the aniline modulator concentration varied from 15-80 equiv. The other amine and aldehyde-based modulators used for knitting prolonged 2D and 3D COF nets include propylamine, 2-aminopyridine, benzaldehyde, and 2,4,6-trimethylbenzaldehyde. ${ }^{178-180}$

A few months ago, the mechanochemical synthetic approach for the synthesis of imine-linked COFs was deeply studied to understand its mechanistic pathway. Four different COFs, viz., COF-LZU1, TbBd-COF, IISERP-COF4 and N-COF, were constructed by liquid-assisted mechanochemical milling of their respective monomers in an X-ray transparent PMMA milling jar. It was evidently shown that by controlling the ratio of liquid additive (dioxane with respect to the reactants) and providing a catalyst suitable for Schiff base formation, very high crystallinity of the framework could be achieved by this method. With this approach, the limited surface area is also enhanced to a good extent when $\mathrm{Sc}(\mathrm{OTf})_{3}$ was utilized for catalyzing the reaction. ${ }^{181}$ The drawbacks of the COF activation process were also reported last year, where the collapse of imine-linked frameworks was reported during high-vacuum activation. The solution to this problem was provided by activating the fragile frameworks with $\mathrm{N}_{2}$ or supercritical $\mathrm{CO}_{2}$ flow activation using an ultrahigh-pressure reactor. ${ }^{182}$

Fig. 17B shows the summarized mechanism, which presents insight into obtaining single crystals of 2D imine-linked COFs via the use of an aniline modulator in large excess (step a), giving the desired product in 30-80 days. However, a polycrystalline material can be obtained in a few hours by using amorphous COF sheets as a precursor (step c), which can be produced within seconds from the monomers (step b).

\section{Structural characterization}

\subsection{Fourier-transform infrared (FTIR) spectroscopy}

This technique is a very basic and widely used technique to detect the functional groups present in any organic system. Almost all organic polymers, particularly COFs, have been characterized using this method due to its easy accessibility and user-friendly approach. This technique can be easily operated and analyzed to obtain subtle results about the functional groups present in materials. It is mostly used to help determine the completion of reactions for covalent organic frameworks, i.e., in the case of Schiff-base imine condensation, the disappearance of the peaks corresponding to an amine and an aldehyde near 3500-3300 and $1690 \mathrm{~cm}^{-1}$, respectively, indicates that the reaction is complete. An example for this is $\mathrm{COF}_{\mathrm{DAAQ}-\mathrm{BTA}-3 \mathrm{DG}}$, which was synthesized via the combination of a ditopic amine unit and a tritopic aldehyde unit. ${ }^{183}$ The fully formed product was witnessed by the appearance of a new $\mathrm{C}-\mathrm{N}$ stretching peak $\left(\sim 1331 \mathrm{~cm}^{-1}\right)$ and the absence of the strong carbonyl stretching frequency near $1695 \mathrm{~cm}^{-1}$ (Fig. 18). This framework was well characterized using other techniques for the confirmation of its synthesis (vide infra).
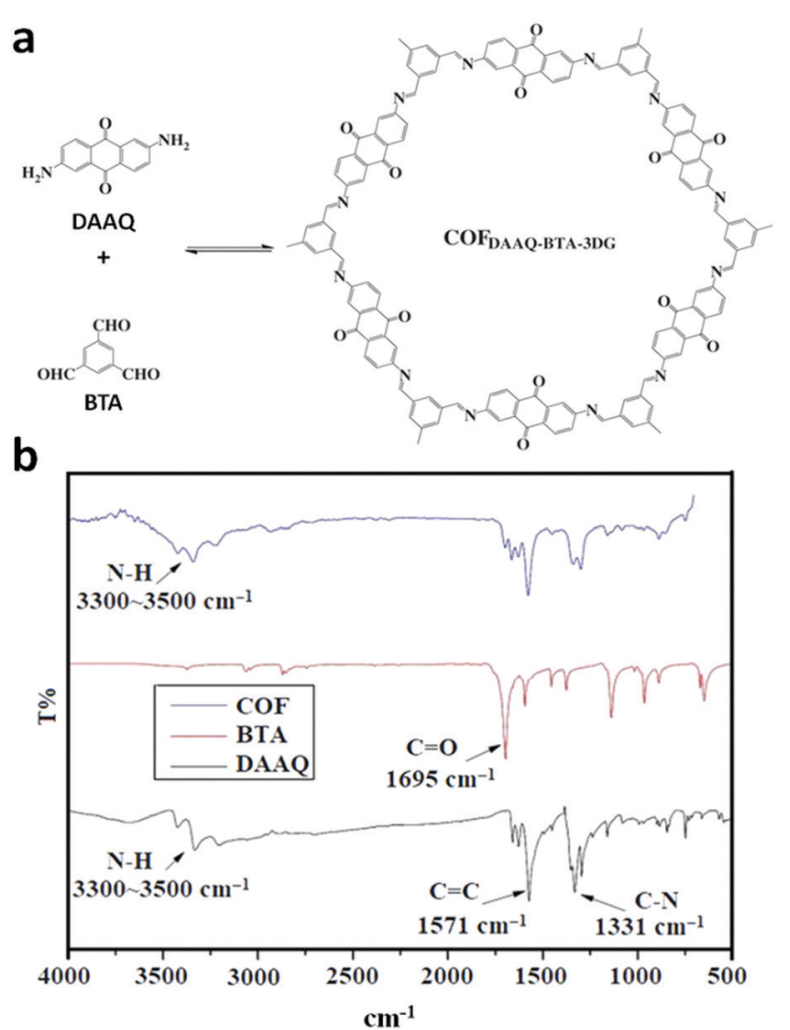

Fig. 18 (a) Structures of DAAQ and BTA monomers and COF and (b) their characterization using FT-IR spectroscopy. Reproduced with permission from ref. 183, Copyright the American Chemical Society (2015). 


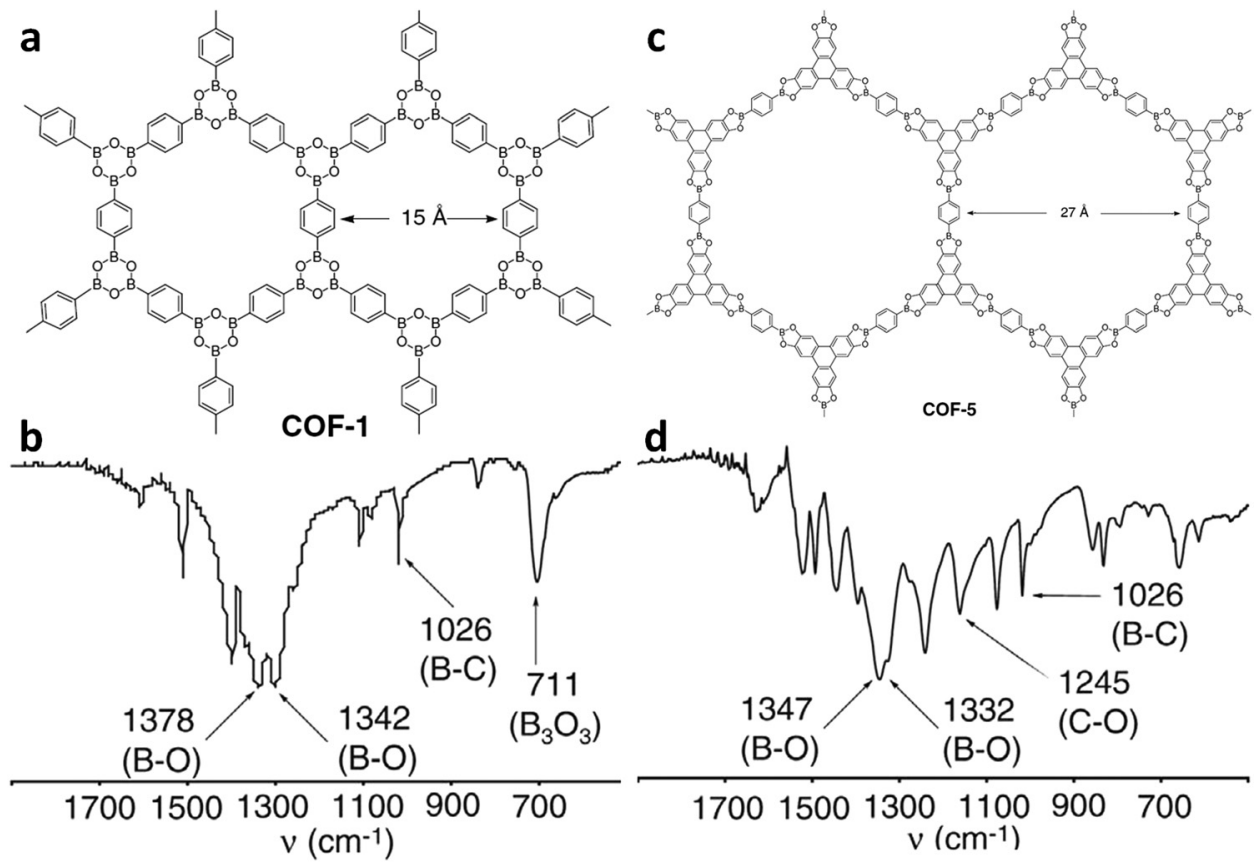

Fig. 19 Condensation reactions of boronic acids used to produce extended COF structures of (a) COF-1 and (c) COF-5. FTIR spectra highlighting the characteristic boron functional group bands of (b) COF-1 and (d) COF-5. Reproduced with permission from ref. 4, Copyright The American Association for the Advancement of Science (2005). Asterisks indicate spinning side-band peaks.

Moreover, as mentioned in Section 4.2, triazine-linked COFs can also be monitored using the FTIR technique by noting the disappearance of the $\mathrm{C}-\mathrm{N}$ stretching frequency for nitriles and appearance of a peak corresponding to imines. The formation of CTF-1 was studied at different reaction times and various reaction temperatures. The intense carbonitrile peak at $2218 \mathrm{~cm}^{-1}$ disappeared, which clearly indicated the successful occurrence of the trimerization reaction. This was further supported by the emergence of a strong absorption peak at $1352 \mathrm{~cm}^{-1}$, corresponding to the formation of a triazine ring. ${ }^{20}$

The boron-containing frameworks have also been well characterized using FTIR spectroscopy. COF-1 and COF-5 (Fig. 19a and c) are linked by boroxine- and boronic ester-linkages, respectively. The FTIR peak at $711 \mathrm{~cm}^{-1}$ increased with respect to the $\left(\mathrm{B}_{3} \mathrm{O}_{3}\right)$ boroxine ring (Fig. 19b), whereas the peak at $1026 \mathrm{~cm}^{-1}$ corresponds to the boronic ester ring B-C stretching frequency (Fig. 19d). ${ }^{4}$ Moreover, the $\mathrm{B}-\mathrm{O}$ stretching frequencies in both cases can be seen at 1378 and $1342 \mathrm{~cm}^{-1}$ for COF-1 and at 1347 and $1332 \mathrm{~cm}^{-1}$ for COF-5.

Similarly, the synthesis of borazine-linked COFs was monitored by the appearance of the B-N stretching frequency near $1500 \mathrm{~cm}^{-1}$ and strong attenuation of the $\mathrm{N}-\mathrm{H}$ and $\mathrm{B}-\mathrm{H}$ frequencies, as shown for BLP-2(H) in Fig. $20 .{ }^{60}$

Considering that COFs are known to form numerous linkages, the formation of these linkages through various reversible reactions has been monitored using FTIR analysis. One of these distinctive linkages is the azidoxy-linked framework, where the nitroso-functionalized monomers dimerize to form a trans-adioxy bond. The POR-COF was constructed using a protected tetrakis(aryl hydroxylamine)porphyrin unit via room temperature

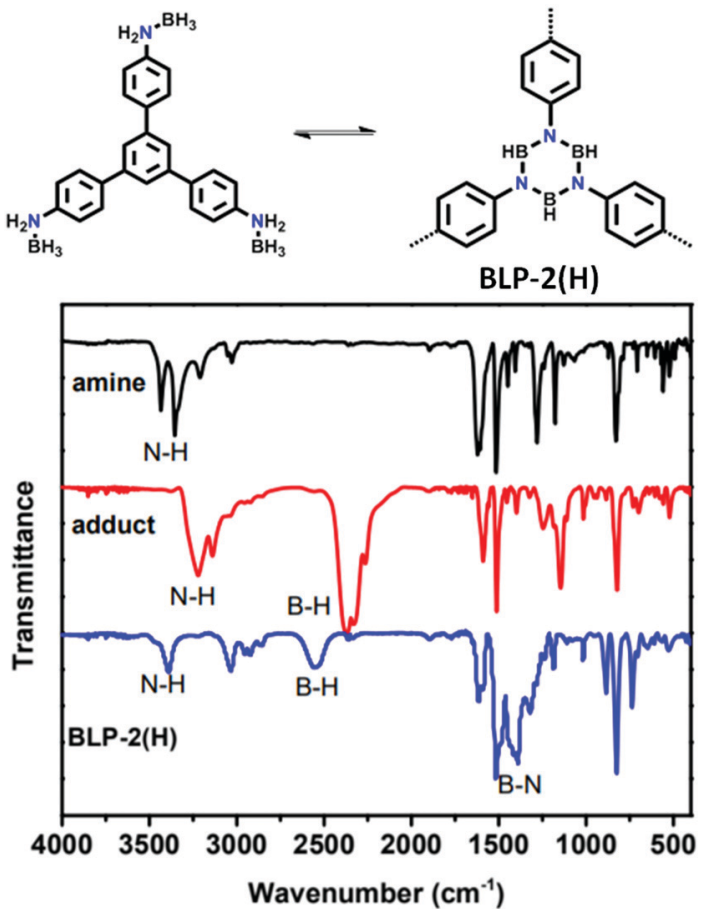

Fig. 20 Structure of BLP-2(H) together with its monomer and its characterization using FT-IR spectroscopy. Reproduced with permission from ref. 60, Copyright The Royal Society of Chemistry (2012).

synthesis (see Section 4.2). ${ }^{35}$ According to the FT-IR spectrum, it was observed that with the propagation of dimer formation, the peaks at $1735 \mathrm{~cm}^{-1}$ for the $\mathrm{C}=\mathrm{O}$ stretching and $2950-2850 \mathrm{~cm}^{-1}$ 
a
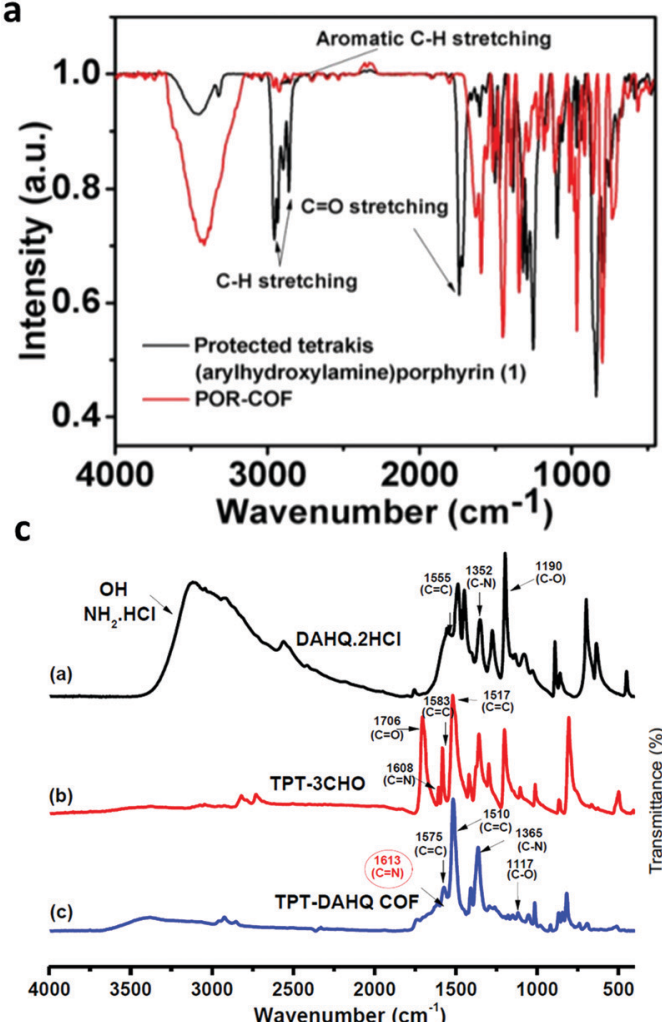
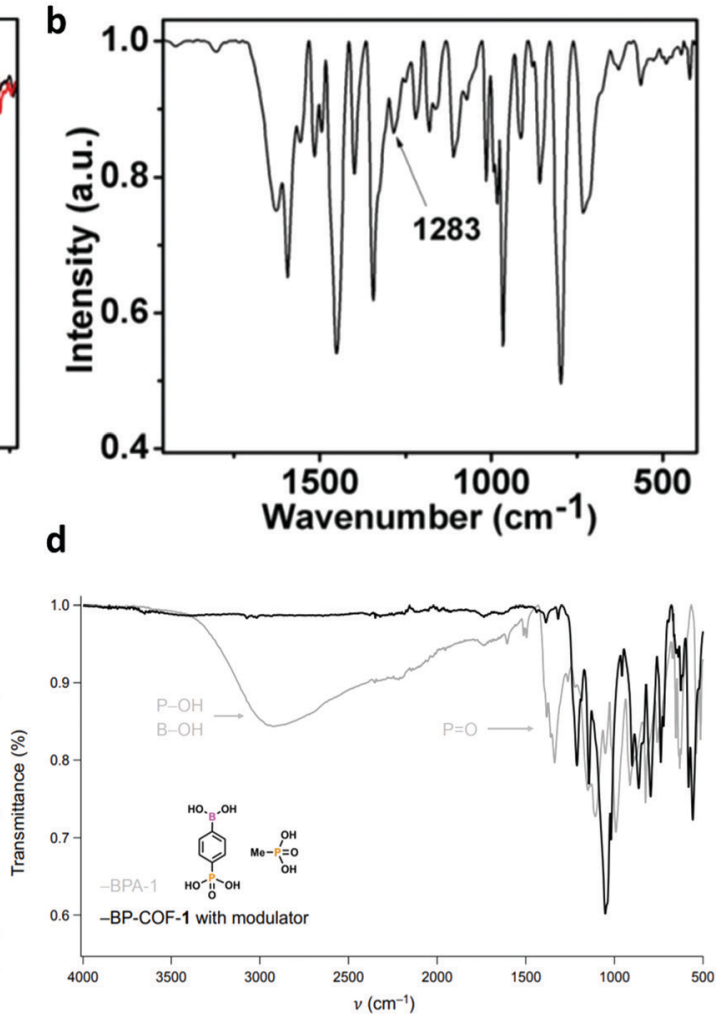

Fig. 21 FT-IR spectra of (a) azidoxy-linked POR-COF and its monomer with its magnified spectrum (b), (c) TAPT-DAHQ COF and its monomers DAHQ. $2 \mathrm{HCl}$ and TAPT-3CHO and (d) BP-COF-1 and its monomer BPA-1. Reproduced with permission from: (a and b) ref. 35, Copyright The Royal Society of Chemistry (2016); (c) ref. 164, Copyright John Wiley and Sons (2019) and (d) ref. 57, Copyright The American Association for the Advancement of Science (2020).

for the $\mathrm{C}-\mathrm{H}$ stretching disappeared (Fig. 21a and b). Moreover, a new peak at around $1283 \mathrm{~cm}^{-1}$ for $\mathrm{N}=\mathrm{N}$ stretching was seen, representing completion of the reaction.

Likewise, the formation of benz-bis(oxazole) was monitored, where the FTIR spectra of the monomers and the product were simultaneously plotted and compared. In Fig. 21c, it can be seen that the peaks corresponding to $\mathrm{C}=\mathrm{N}\left(1608 \mathrm{~cm}^{-1}\right), \mathrm{C}=\mathrm{C}$ (1583-1517 $\left.\mathrm{cm}^{-1}\right)$ and $\mathrm{C}-\mathrm{O}\left(1706 \mathrm{~cm}^{-1}\right)$ for the TPT-3CHO monomer and that for $\mathrm{C}-\mathrm{N}$ bending $\left(1352 \mathrm{~cm}^{-1}\right), \mathrm{C}=\mathrm{C}$ $\left(1555 \mathrm{~cm}^{-1}\right), \mathrm{C}-\mathrm{O}\left(1190 \mathrm{~cm}^{-1}\right)$ and phenolic $-\mathrm{OH}$ and $\mathrm{NH}_{3} \mathrm{Cl}$ $\left(\sim 3200 \mathrm{~cm}^{-1}\right)$ for the DAHQ.2HCl monomer disappeared during the reaction, whereas peaks for $\mathrm{C}=\mathrm{N}\left(1613 \mathrm{~cm}^{-1}\right)$ and $\mathrm{C}-\mathrm{O}$ $\left(117 \mathrm{~cm}^{-1}\right)$ appear. This is attributed to the formation of a benzobisoxazole ring. ${ }^{164}$

The synthesis of the highly connected BP-COF-1 using BP-1 was also studied using FTIR spectroscopy (Fig. 21d). The broad peak corresponding to free $-\mathrm{OH}$ groups and sharp $\mathrm{P}=\mathrm{O}$ stretching in the monomer unit were attenuated completely after completion of the reaction. ${ }^{57}$

Monitoring the formation of the $\beta$-ketoenamine linkage using FT-IR was reported in 2015, which compared the fabrication of the TpBDH COF from 1,3,5-triformylphloroglucinol (Tp) and pyromellitic- $N, N^{\prime}$-bisaminoimide (BDH) units (Fig. 22). ${ }^{149}$ The appearance of peaks at 1583 and $1273 \mathrm{~cm}^{-1}$ correspond to the $\mathrm{C}=\mathrm{C}$ and $\mathrm{C}-\mathrm{N}$ stretching, respectively, which match that of

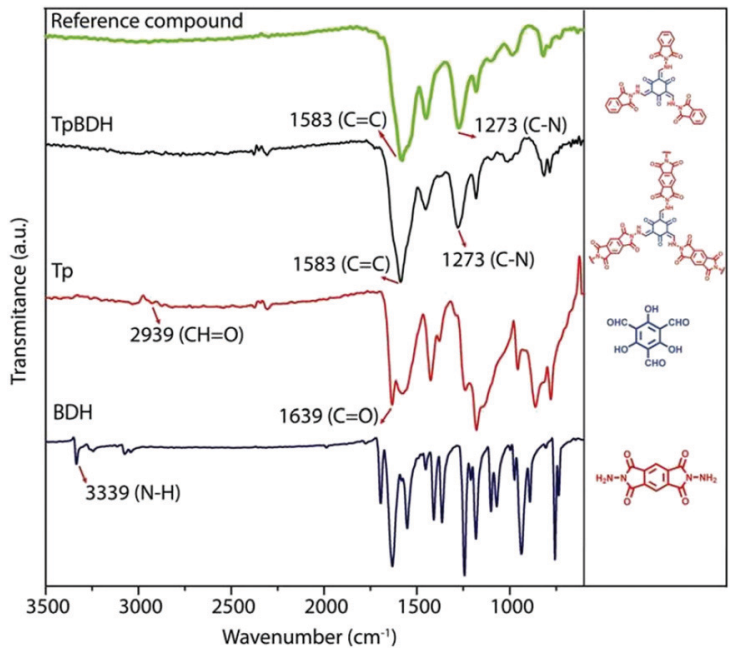

Fig. 22 FT-IR spectra of TpBDH COF with its monomers, Tp and BDH, and the reference compounds. Reproduced and adapted with permission from ref. 149, Copyright The Royal Society of Chemistry (2015)

the reference compound. Conversely, the monomer peaks for $\mathrm{N}-\mathrm{H}$ and $\mathrm{C}=\mathrm{O}$ stretching are fully attenuated in the product.

Monitoring thin film synthesis from bulk COFs. COF thin films have been proven to be emerging candidates for sensing applications. Their synthesis from the bulk powder demands 


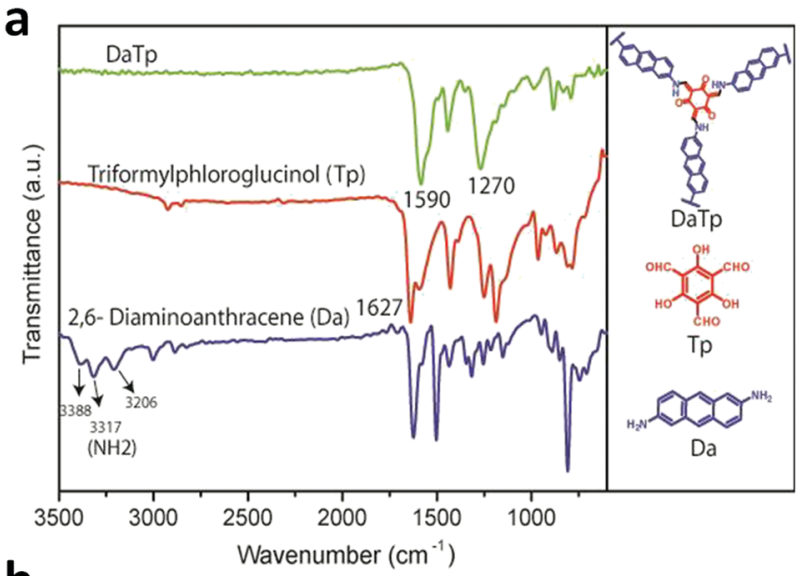

b

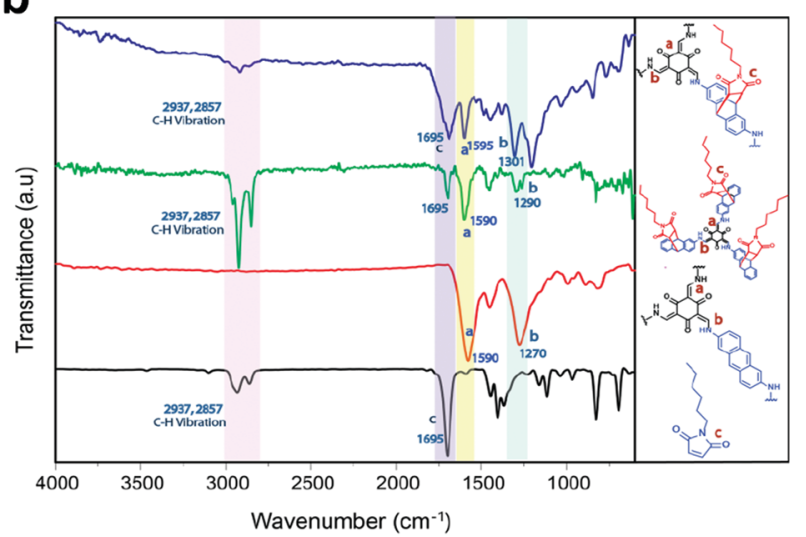

Fig. 23 FT-IR spectra of (a) DaTp COF and its monomers, Tp and Da and (b) DaTp-CON with $N$-hexylmaleimide. Reproduced with permission from ref. 110, Copyright John Wiley and Sons (2016).

the choice of solvents and other conditions, as mentioned in Section 4.1. FT-IR spectroscopy is employed as the first tool to validate the powder-to-film conversion. For example, the $\beta$-ketoenamine-linked DaTp COF was exfoliated chemically to yield DaTp-CON sheets using $N$-hexylmaleimide at $160^{\circ}$ for 24 h. ${ }^{110}$ Initially, the synthesis of DaTp COF was confirmed by comparing its stretching frequencies with the monomers, 2,6-diaminoanthracene (Da) and 2,4,6-triformylphloroglucinol (Tp) (Fig. 23a). The complete disappearance of the peaks at 3388,3317 and $3206 \mathrm{~cm}^{-1}$, corresponding to the $\mathrm{NH}_{2}$ group in $\mathrm{Da}$ and that at $1627 \mathrm{~cm}^{-1}$ for the -CHO group in Tp, and the characteristic $\mathrm{C}=\mathrm{C}$ and $\mathrm{C}-\mathrm{N}$ peaks at 1590 and $1270 \mathrm{~cm}^{-1}$ for the bulk COF confirmed the reaction progress. Afterwards, the exfoliated DaTp-CON showed some additional stretching frequency bands at 2937, 2857 and $1695 \mathrm{~cm}^{-1}$, corresponding to the $\mathrm{C}-\mathrm{H}$ and $\mathrm{C}=\mathrm{O}$ groups from the alkyl chain and imide unit of $N$-hexylmaleimide (Fig. 23b).

Monitoring linkage transformation via post-synthetic modification. Post-synthetic modification of covalent organic frameworks is an emerging field, which has improved the applicability of these materials for numerous applications, one of which is sensing. Imine-linked COFs, viz. TPB-TP-COF (1) and 4PE-1P-COF (2), were subjected to post-synthetic modification via oxidation of the imine to amide linkages using mild reaction conditions of

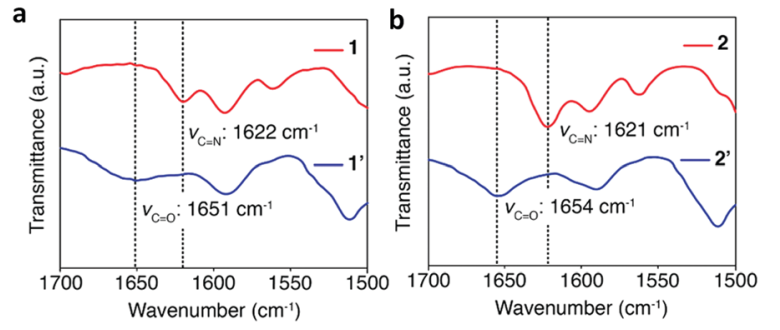

Fig. 24 FT-IR spectra of imine-linked (a) TPB-TP COF (1) and (b) 4PE-1PCOF (2) in red with their oxidized products amide-linked (a) TPB-TP COF $\left(\mathbf{1}^{\prime}\right)$ and (b) 4PE-1P-COF (2') in blue. Reproduced with permission from ref. 184, Copyright the American Chemical Society (2016).

$\mathrm{NaClO}_{2} / 2$-methyl-2-butene/1,4-dioxane with $\mathrm{AcOH}$ catalyst and left to stand at room temperature for 2 days. ${ }^{184}$ The amidelinked frameworks were denoted as $\left(\mathbf{1}^{\prime}\right)$ and $\left(\mathbf{2}^{\prime}\right)$, respectively. The appearance of FTIR stretching peaks at $1651 \mathrm{~cm}^{-1}$ (for $\mathbf{1}^{\prime}$ ) and $1654 \mathrm{~cm}^{-1}$ (for $2^{\prime}$ ) with respect to the amide $(\mathrm{C}=\mathrm{O})$ group and disappearance of the $\mathrm{C}=\mathrm{N}$ imine stretching peaks at $\sim 1622 \mathrm{~cm}^{-1}$ (for $\mathbf{1}^{\prime}$ and $\mathbf{2}^{\prime}$ ) support the linker transformation process (Fig. 24).

A similar study was done to strengthen the imine linked TTICOF by post-synthetic modification to form the thiazole-linked TTT-COF at high thermal conditions (vide Section 4.2). ${ }^{170}$ The disappearance of the peak at $1627 \mathrm{~cm}^{-1}$ corresponds to the $\mathrm{C}=\mathrm{N}$ imine stretching in TTI-COF and the emergence of a new $\mathrm{C}=\mathrm{N}$ stretching for the thiazole ring can be seen at $1609 \mathrm{~cm}^{-1}$.

As exemplified above, this is the foremost and a very basic tool that can be used in different aspects for functional group analysis in a framework.

\subsection{Solid-state magic angle spinning (ss-MAS) nuclear magnetic resonance (NMR) spectroscopy}

Covalently-bonded organic polymers are usually insoluble in common solvents, which make their analysis difficult using the NMR technique given that it requires high solubility of the sample in deuterated solvents. Therefore, magic-angle spinning of the solid-state sample is done at an angle $54.74^{\circ}$ with respect to the magnetic field during NMR analysis. This technique is used for monitoring the formation of COFs by comparing the NMR peaks of the polymer with their respective monomers. The first set of COFs was comprised of B, C, O and N-atoms, where ${ }^{11} \mathrm{~B},{ }^{13} \mathrm{C}$ and ${ }^{15} \mathrm{~N}$ NMR spectroscopy were utilized to corroborate the framework structure. Recently, ${ }^{31} \mathrm{P}$ and ${ }^{29} \mathrm{Si}$ NMR spectroscopy were exploited for their characterization with the incorporation of $\mathrm{P}$ and $\mathrm{Si}$ atoms in the linkages. ss-MAS NMR was first implemented for COF- 1 and COF-5 to study the completion of their reaction. The ${ }^{11}$ B-MAS NMR spectra for both COFs do not match that of the monomer benzenediboronic acid (BDBA) and totally matches with their model compounds. This indicates the formation of the boroxine ring in COF-1 and boronic ester in COF-5 (Fig. 25a and b), respectively. ${ }^{4}$

${ }^{13} \mathrm{C}$ and ${ }^{15} \mathrm{~N}$ CP-MAS NMR spectra were used to detect the formation of the triazine ring in a covalent triazine framework, $v i z$. CTF- $0 .{ }^{13} \mathrm{C}$ NMR indicated three major peaks at $\delta=167$, 

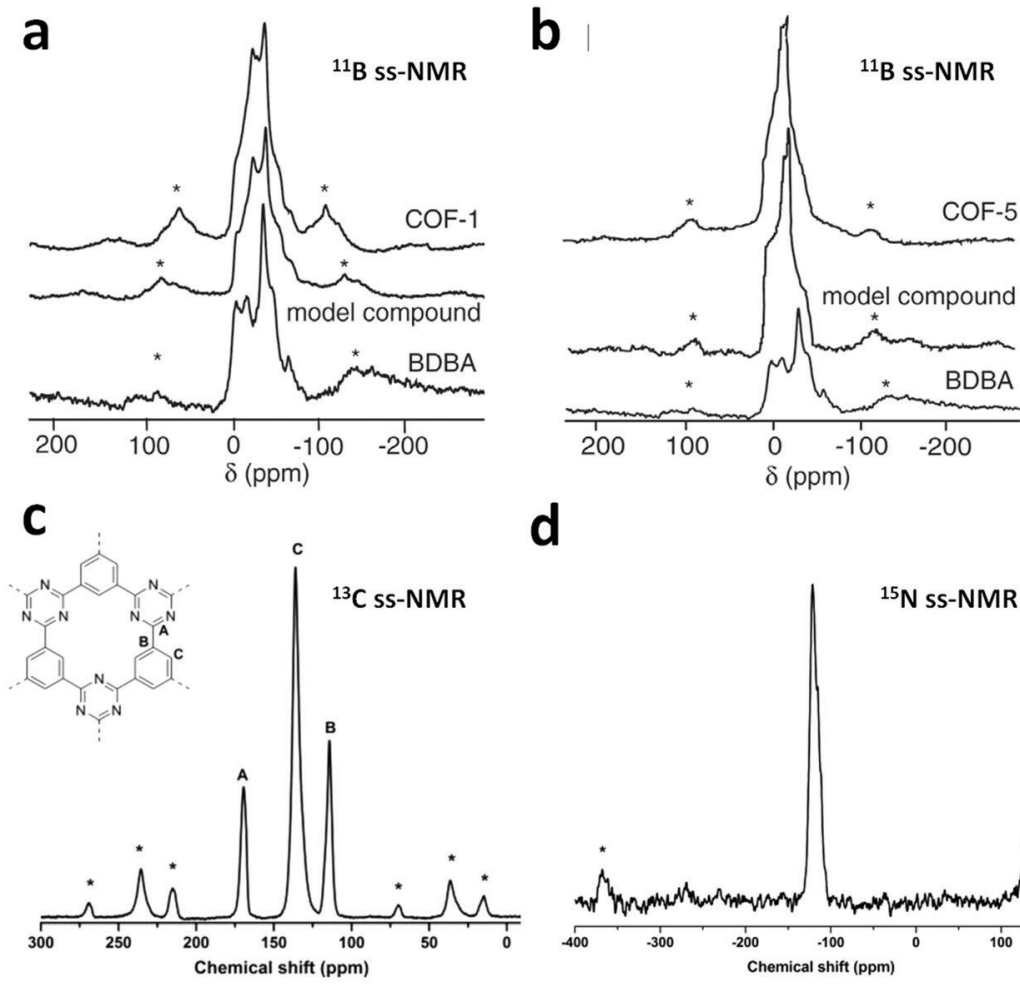

d
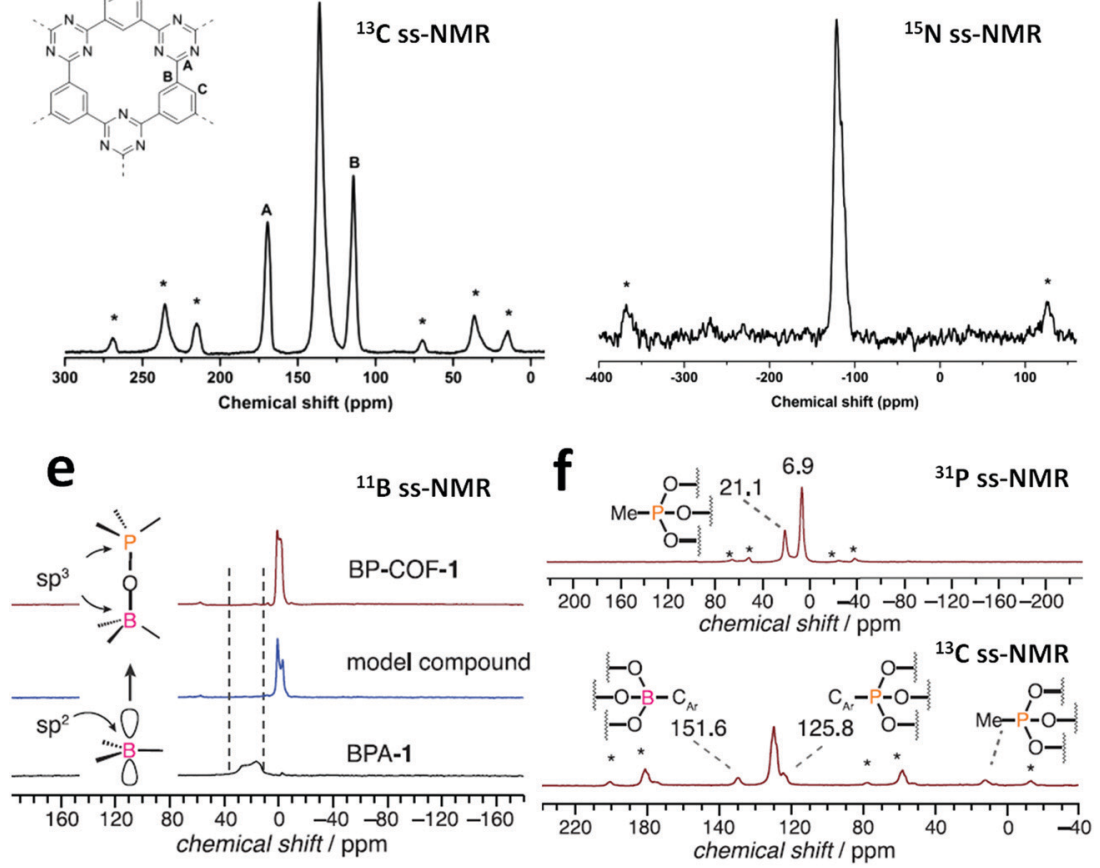

Fig. $25{ }^{11}$ B magic-angle spinning NMR spectra of (top) COF, (middle) model compound and (bottom) BDBA for (a) COF-1 and (b) COF-5. Reproduced and adapted. ${ }^{13} \mathrm{C}$ CP-MAS solid-state NMR (c) and ${ }^{15} \mathrm{~N}$ CP-MAS solid-state NMR (d) of CTF-0. (e) Overlay of the ${ }^{11} \mathrm{~B}$ solid-state (ss) NMR spectra of BPCOF-1, a molecular boro-phosphonate model compound and BPA-1. (f) ${ }^{31} \mathrm{P}$ and ${ }^{13} \mathrm{C}$ NMR spectra of BP-COF-1. Me, methyl. Asterisks denote spinning sidebands. Reproduced with permission from: ( $a$ and b) ref. 4, Copyright The American Association for the Advancement of Science (2005); (c and d) ref. 19. Copyright the American Chemical Society (2013) and (e and f) ref. 57, Copyright The American Association for the Advancement of Science (2020).

136 and $114 \mathrm{ppm}$, which correspond to the three $\mathrm{sp}^{2}$ carbons (the deshielded peak for the triazine ring and other two for the phenyl ring) and no peak was observed at $\delta=110 \mathrm{ppm}$, ruling out the possibility of any residual $\mathrm{CN}$ group from the monomer unit (Fig. 25c and d). ${ }^{19}$ In addition, the ${ }^{15} \mathrm{~N}$ ssNMR spectrum helped to identify the triazine moiety, with a peak observed at $-121 \mathrm{ppm}$ for the triazine-ring $\mathrm{N}$-atom.

Similarly, ${ }^{11} \mathrm{~B}$-MAS NMR spectroscopy was used to validate the complete utilization of monomers in the polycubane-based BP-COF-1 (Fig. 25e).$^{57}$ The single doublet of the framework near $0 \mathrm{ppm}$ for the COF is consistent with the model compound, whereas this peak was not observed for $\mathrm{sp}^{2}$-hybridised boron in the monomer BPA-1. Moreover, ${ }^{31} \mathrm{P}$ and ${ }^{13} \mathrm{C}$ ssNMR spectroscopy were also used to confirm the reaction (Fig. 25f). The ${ }^{31} \mathrm{P}$ NMR spectrum of BP-COF-1 showed two peaks at 6.9 and $21.1 \mathrm{ppm}$, indicating the presence of two distinct phosphorous atoms in the framework. The less intense peak at $21.1 \mathrm{ppm}$ represents the methylphosphates present at the crystallite edges, whereas the more shielded NMR signal at $6.9 \mathrm{ppm}$ represents the $\mathrm{C}_{\mathrm{Ar}}-\mathrm{P}$ unit. Conversely, the ${ }^{13} \mathrm{C}$ CP-MAS NMR spectrum displays peaks in the aromatic region between 151.6 and $125.8 \mathrm{ppm}$, which correspond to the aromatic carbon bonded to the boron and phosphorus atoms, respectively.

Silicon-containing frameworks have been synthesized to incorporate ionic nature in COFs (see Section 4.2). Therefore, for their characterization, ${ }^{29} \mathrm{Si}$ CP-MAS ssNMR spectroscopy has been exploited. The synthesis of an anionic SiCOF was validated using ${ }^{29} \mathrm{Si}$ NMR spectroscopy, which showed a single peak at $-143 \mathrm{ppm}$, corresponding to the hexacoordinated Si-atom in the framework. ${ }^{165}$ However, no peak for the TMOS monomer at 
a

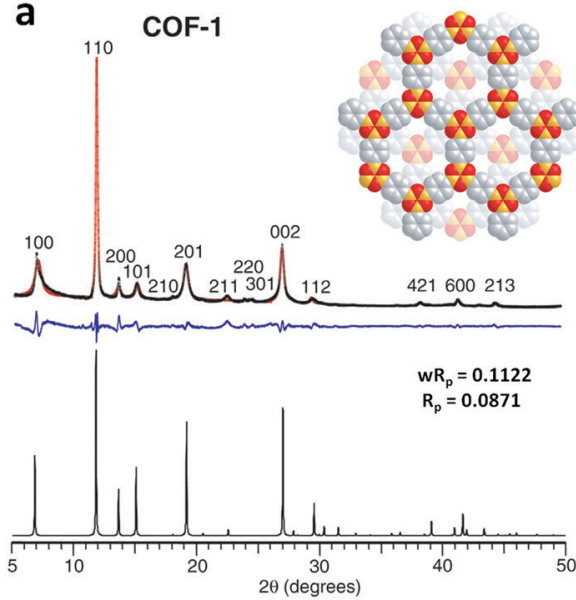

C:

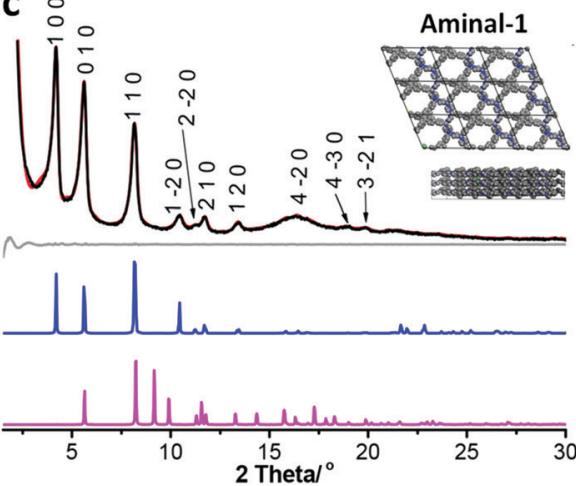

b
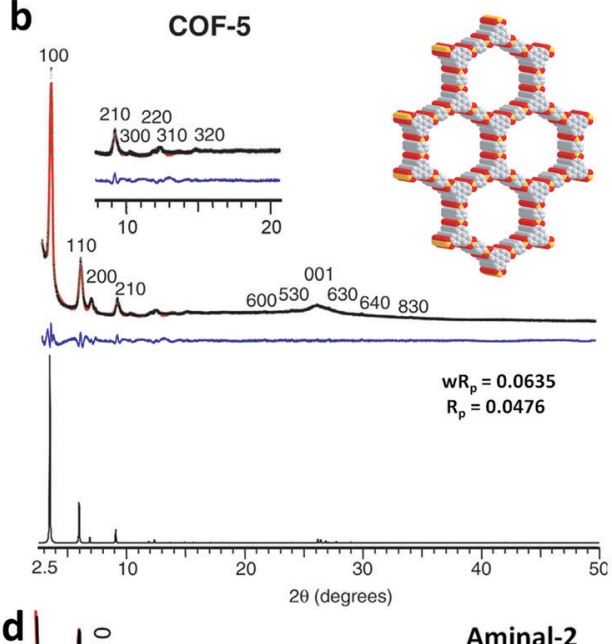
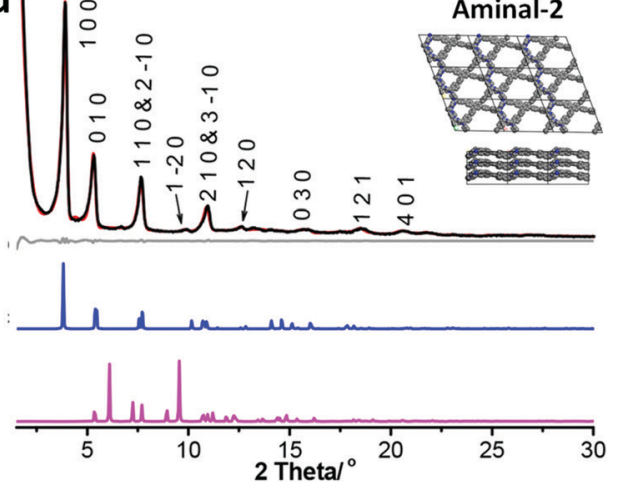

Fig. 26 X-ray analysis of (a) COF-1 and (b) COF-5 with the observed pattern in black, the refined profile in red, and the difference plot in blue (observed minus refined profiles). The bottom trace is the calculated PXRD pattern from Cerius. ${ }^{2}$ Experimental (black) and refined (red) PXRD patterns of (c) aminalCOF-1 and (d) aminal-COF-2. Difference plot between the experimental and refined PXRD patterns (grey) and simulated PXRD patterns for eclipsed (blue) and staggered (pink) structures. Inset is the perspective and top views of the structural representation of both COFs in eclipsed stacking. Reproduced with permission from: (a and b) ref. 4, Copyright The American Association for the Advancement of Science (2005) and (c and d) ref. 38, Copyright the American Chemical Society (2019).

$-79 \mathrm{ppm}$ was seen. Considering that the COF was anionic and stabilized by $\mathrm{Li}^{+}$and $\mathrm{Na}^{+}$ions, the rarely utilized ${ }^{7} \mathrm{Li}$ and ${ }^{23} \mathrm{Na}-$ MAS NMR spectra were recorded, showing peaks at $-1 \mathrm{ppm}$ for SiCOF-Li and $-24 \mathrm{ppm}$ for SiCOF-Na, respectively. This is a unique example of the use of Na and Li NMR spectroscopy.

\subsection{X-ray diffraction (XRD)}

As mentioned above, COFs are synthesized in the form of small crystallites/powders, highly ordered thin films and small single crystals. Therefore, X-ray diffraction is an extensively exploited analytical technique used to elucidate the crystallographic structure of these materials. The powders and films are characterized using PXRD (small/wide angle X-ray diffraction) supported by theoretically simulated peaks, whereas single crystals with good mosaic structures are characterized using the SCXRD technique.

5.3.1 Powder X-ray diffraction (PXRD) supported by theoretical simulations. Generally, the synthetic techniques applied to produce COFs yield fine powders, which are diffracted using the PXRD technique. The intense and sharp diffraction peaks fulfilling Bragg's law indicate high order of crystallinity in the frameworks. In addition, a simulated study is conducted on the framework using different basis sets and hybrid functionals, which generate the simulated pattern and structure of the COFs. Various important parameters of the porous organic framework such as atom-coordinates, unit cell parameters, space group, interlayer distance and the type of stacking for 2D COFs, degree of interpenetration in 3D COFs, framework packing, and pore shape and size can be determined using this technique. Moreover, this method also indicates the homogeneity of the sample, which can also be confirmed by microscopic techniques (vide Section 5.4).

Structure determination. The first highly crystalline COFs were the boron-containing COF-1 and COF-5, which were characterized by PXRD, giving sharp peaks corresponding to different $\left(\begin{array}{lll}h & k & l\end{array}\right)$ planes, as shown in Fig. 26a and b, respectiverly. ${ }^{4}$ To confirm these planes, the extended structures of both frameworks were modelled and their simulated powder patterns were calculated. The simulated patterns were found to be well fitted with the experimental patterns, indicating the formation of staggered $\mathrm{AB}$ stacked and eclipsed $\mathrm{AA}$ stacked frameworks for COF-1 and COF-5 with the hexagonal lattice 
space groups of $\mathrm{P6}_{3} / \mathrm{mmc}$ and $\mathrm{P6} / \mathrm{mmm}$, respectively. The honeycomb framework sets in the gra and bnn network with and interlayer spacing of 3.328 and $3.460 \AA$ for COF-1 and COF-5, respectively.

Aminal-linked COFs, viz. aminal-1 and aminal-2, diffract to give very sharp and intense peaks, corresponding to the (100), (010) and (110) facets, which match well with the simulated patterns with fairly low fitting parameters (Fig. 26c and d),

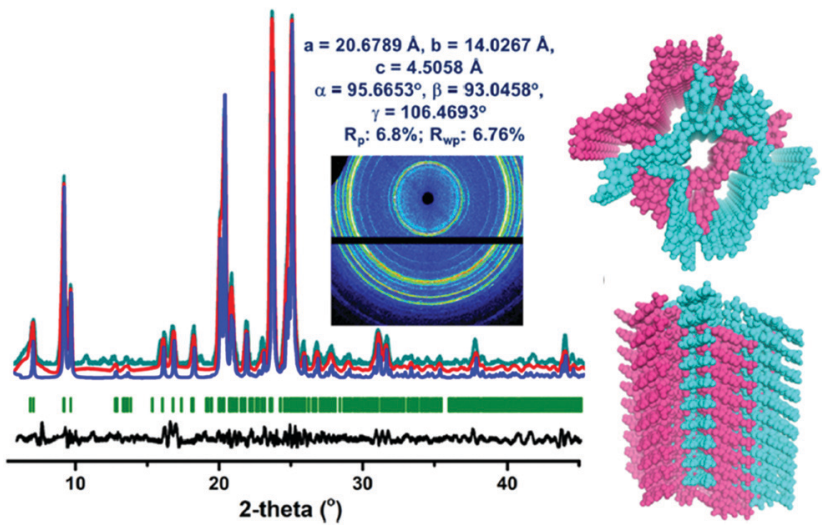

Fig. 27 Structural analysis of ANCOF: (left) experimental PXRD pattern (blue), simulated PXRD pattern (cyan), and its fit by Pawley refinement (red) with a minimum difference (black line). (right) Structures of ANCOF obtained from the Rietveld refinement; $A B$ stacking is shown in cyan and pink color. Reproduced with permission from ref. 154, Copyright the American Chemical Society (2020). respectively. ${ }^{38}$ Both frameworks show eclipsed (AA) stacking in the triclinic $P 1$ space group with the rarely fabricated $2 \mathrm{D}$ cpi topology (vide supra, Section 2.1). It was observed that the two COFs possess piperazine units, which exist in the chair-form, resulting in an increase in the interlayer distance (beyond $6 \AA$ ) and reduction in aromatic $\pi-\pi$ interactions.

Similarly, a nitrogen-rich azine-linked COF with triazine cores, namely ANCOF, showed very sharp and intense diffraction peaks in small/wide angle PXRD, which are consistent with the simulated pattern calculated using the Pawley and Rietveld refinement methods with good profile-fitting values. ${ }^{154}$ The framework was set in the triclinic $P \overline{1}$ space group with an $\mathrm{AB}$ type interpenetrated arrangement, as shown in Fig. 27. The interlayer separation was calculated to be $4.5 \AA$.

The two imine-linked COFs, 2,3-DhaTta and 2,3-DhaTab, were found to have the AA stacked pattern (Fig. 28b and c), respectively. ${ }^{79}$ As mentioned in Section 3.2.1, the strong $\pi-\pi$ interactions of 2,3-DhaTta COF due to its planar building units were clearly depicted from its high intensity $(100) /(001)$ peak ratio of 46.21 in comparison to that of the 2,3-DhaTab COF, which is 5.01 (Fig. 28a and d), respectively. The simulated results showed the greater $\pi-\pi$ stacking energy of $-1634.2 \mathrm{~kJ} \mathrm{~mol}^{-1}$ for the former COF in comparison to that of the latter of $-1578.6 \mathrm{~kJ} \mathrm{~mol}^{-1}$. The deviation from planarity of the phenyl-core 2,3-DhaTab COF possibly indicates the improper growth of the material along the c-axis, which disrupts the proper stacking and yields bent crystallites with a lower BET surface area.
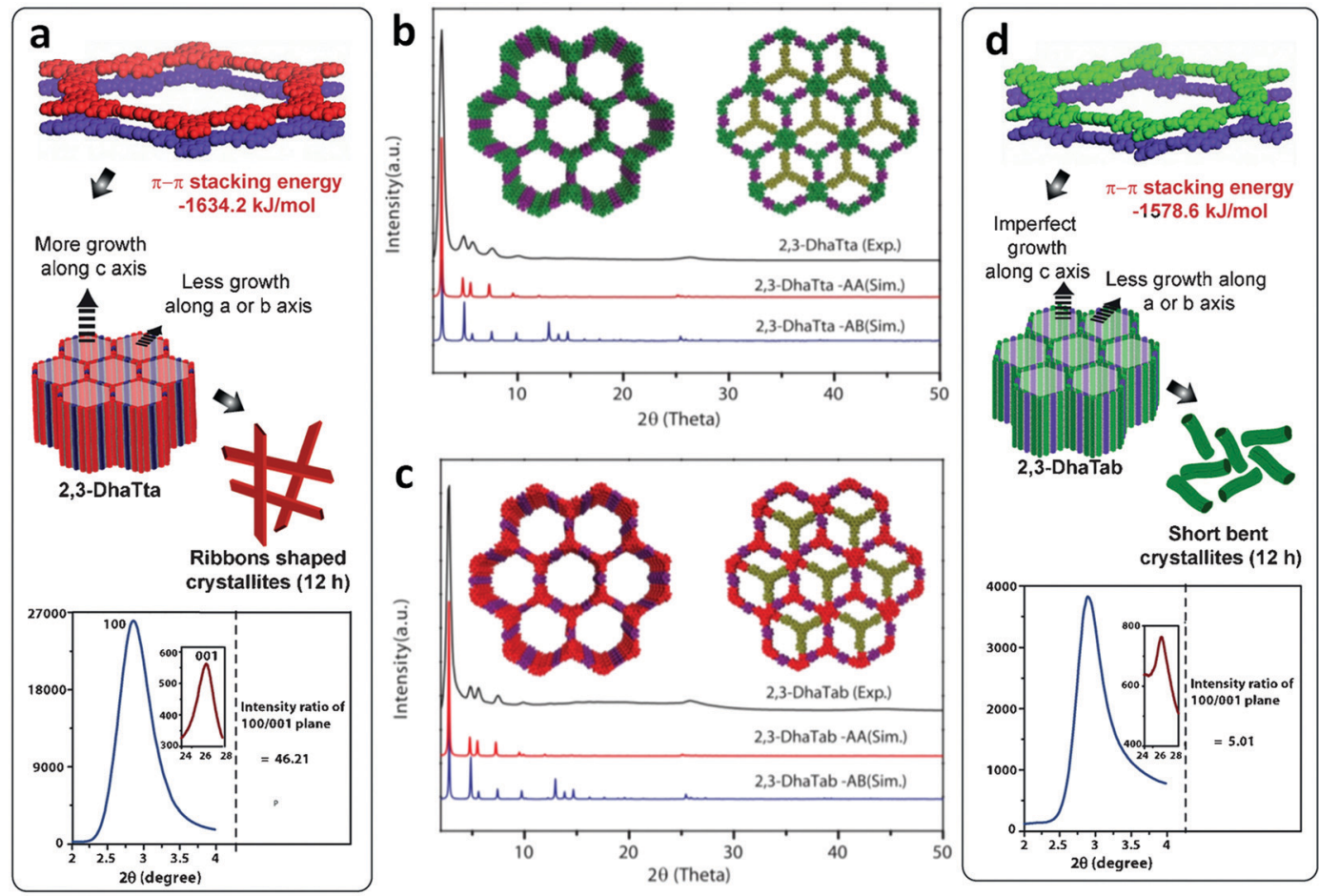

Fig. 28 Mechanism for the formation of ribbon (a) and hollow sphere (d) for CPP 2,3-DhaTta, and 2,3-DhaTab, respectively. PXRD patterns of assynthesized (b) 2,3-DhaTta and (c) 2,3-DhaTab (black) compared with the eclipsed (red) and staggered (blue); eclipsed (left) and staggered (right) stacking models shown in the inset. Reproduced and adapted with permission from ref. 79, Copyright John Wiley and Sons (2016). 
Optimization of synthetic conditions. It was discussed in the previous sections (particularly, Section 4) that optimization of the reaction conditions for the synthesis of COFs with prolonged periodicity, in-built thermal, chemical and water stability and functionalization is a very tedious task. A large number of factors must be thoroughly considered during their synthesis, which makes the process laborious and time-consuming. However, the utilization of appropriate characterization techniques to monitor the reaction progress under different conditions must be considered to save time and gain a better understanding. This case was observed when the TFPC-NDA COF was synthesized under two different reaction conditions (Fig. 29a). ${ }^{24}$ The use of an acid catalyst to activate the aldehydic functional group under solvothermal conditions has been done for long time, but in this rarely seen case, the COF was synthesized without the use of the AcOH catalyst. The PXRD pattern showed intense peaks when the acid was not used as a catalyst during the solvothermal synthesis, whereas very broad peaks were observed when the synthesis was conducted with acid, indicating the amorphous nature of the COF. Furthermore, the COF was utilized for VOC and TNP sensing (see Section 6.2) and its stability in each application was validated using the PXRD technique, as discussed further below.

Additionally, a few in situ PXRD experiments have been performed to understand the formation of the framework. This 'in situ' approach is beneficial to check the reaction requirements to obtain a particular framework from amorphous organic a

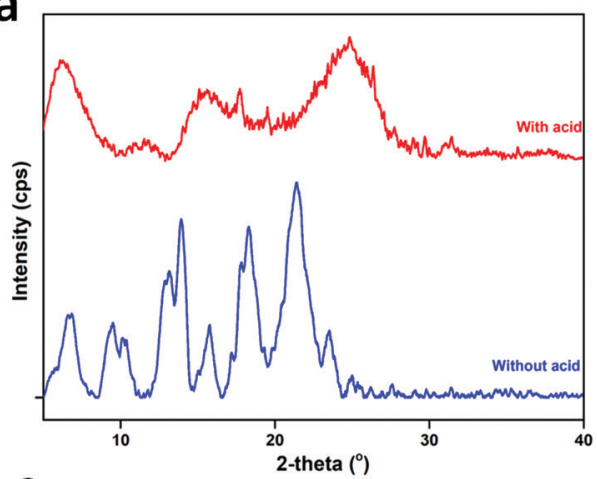

C
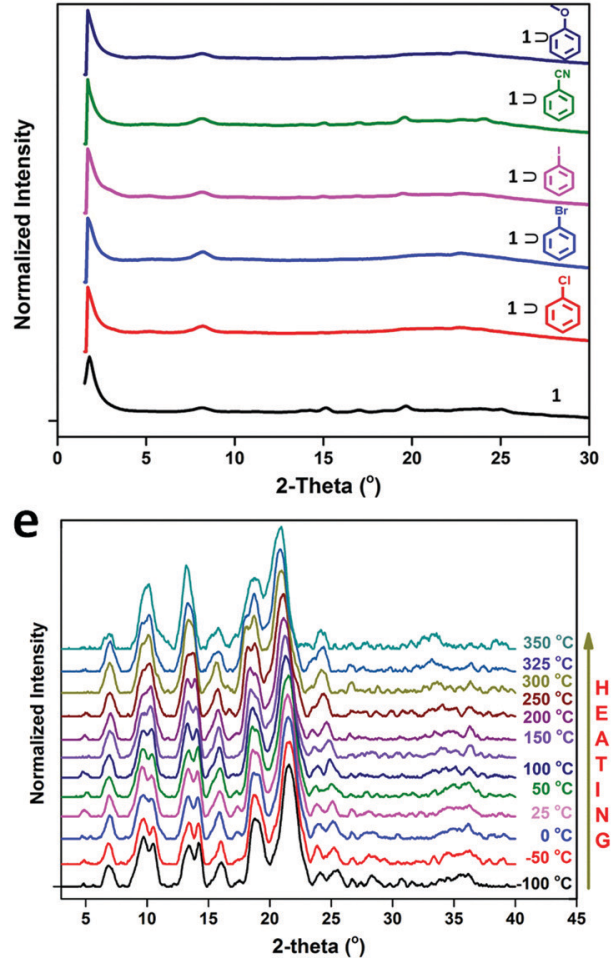

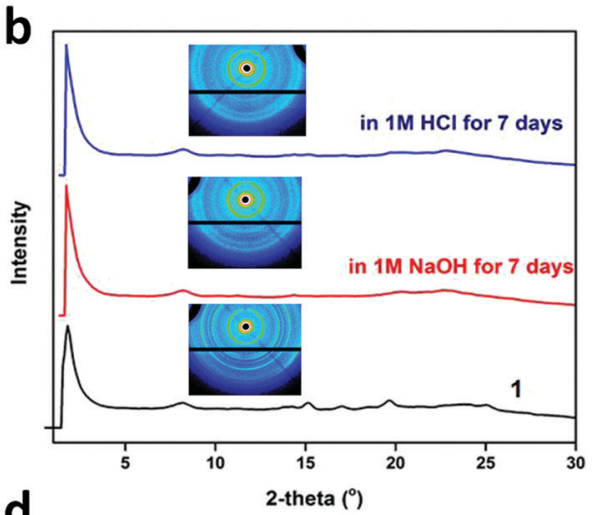

d
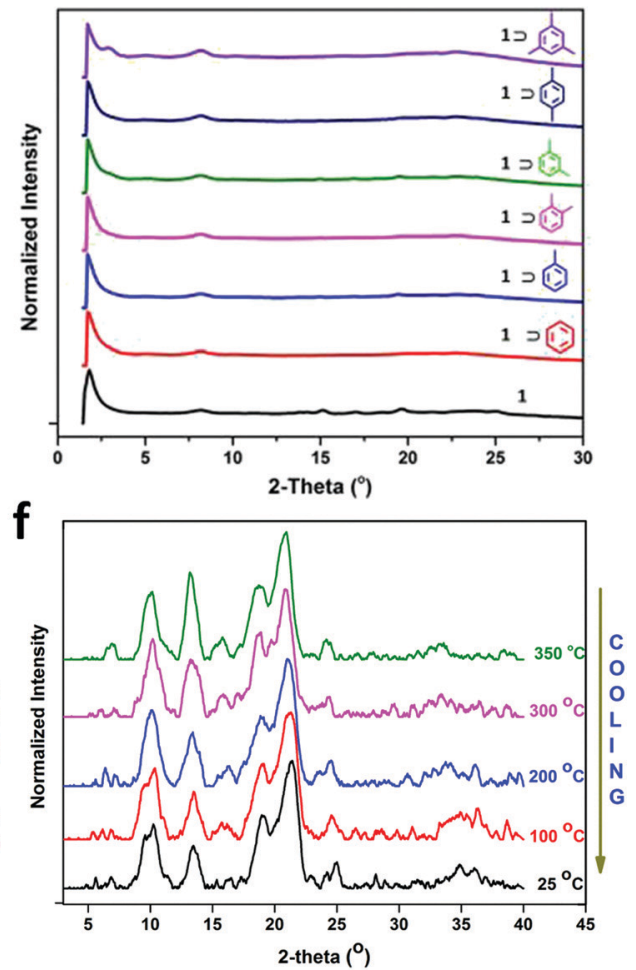

Fig. 29 (a) PXRD patterns of TFPC-NDACOF prepared without an acid and its analogue prepared in the presence of an acid. (b) WAX patterns of TFPCNDACOF before and after immersion in $1 \mathrm{M} \mathrm{NaOH}$ and $1 \mathrm{M} \mathrm{HCl}$ for 7 days (inset: $2 \mathrm{D}$ WAX images). (c and d) WAX patterns showing that the crystallinity of TFPC-NDACOF was maintained after the absorption of different electron-withdrawing and electron-donating aromatic VOCs, respectively. Variabletemperature powder diffraction patterns of TFPC-NDA COF upon (e) heating from $-100{ }^{\circ} \mathrm{C}$ to $350{ }^{\circ} \mathrm{C}$ and (f) cooling to $25{ }^{\circ} \mathrm{C}$. Reproduced with permission from ref. 24, Copyright The Royal Society of Chemistry (2018). 
polymers. For instance, during the study of the mechanochemical synthesis of four COFs, i.e., COF-LZU1, TbBd-COF, IISERP-COF4 and N-COF (vide supra, Section 4.3), the in situ time-resolved synchrotron PXRD studies revealed the appearance of the first peak corresponding to the 100 plane in 3, 10, 10 and $4 \mathrm{~min}$, respectively. ${ }^{181}$ Moreover, during the course of the reaction, the peaks intensified and reached the highest value after a certain time. At this time, the peak intensity matched that from the ex situ PXRD measurements. Additionally, the ex situ PXRD data of the COF-LZU1 intermediate, which crystallizes in the triclinic $P \overline{1}$ space group, revealed that there is one triple imine unit and one-half dioxane molecule in the asymmetric unit.

Stability confirmation. For their use in various applications, COFs must be stable in different conditions. Particularly, in the case of sensing, their stability in the presence of different analytes is necessary to avoid collapse of the framework. A large number of systems can act as analytes, such as nitro-rich explosives, metal ions and anions, acids, bases, small molecules such as ammonia, and various organic solvents. Besides, the stability of the framework at different temperatures can also be investigated using the variable-temperature PXRD (VT-PXRD) technique.

Several examples of this have been reported in the literature, two of which are the $\beta$-ketoenamine-linked TpPa- 1 and TpPa-2 COFs. Their exceptionally high thermal, water and chemical stability were confirmed by the PXRD technique, as shown in Fig. 30. ${ }^{67}$ Other analytic methods such as FESEM, BET and TGA analysis help in getting more detailed information regarding the retention of morphology, high surface areas before and after treatment with different environments and the decomposition temperature, respectively (vide infra).

Another example, continuing from the previous subsection, is TFPC-NDA COF, which is a semi-flexible COF synthesized from 1,3,5-tri(4-formylphenoxy)cyanurate (TFPC) and naphthalene diamine (NDA). The COF was highly stable in various acid/basic conditions ( $1 \mathrm{M} \mathrm{HCl}$ and $1 \mathrm{M} \mathrm{NaOH}$ solutions for 7 days) based on its wide angle PXRD patterns (Fig. 29b). ${ }^{24}$ The 2D WAX image showed the first diffraction peak from the WAX pattern. This highly stable COF was exploited for sensing different analytes, in which its stability was checked. The intact WAX patterns of the COF in different electron-poor and electron-rich (Fig. 29c and d) aromatic analytes confirmed its chemical stability, respectively. In addition, the thermal stability of the COF in a broad temperature range of $350{ }^{\circ} \mathrm{C}$ to $-100{ }^{\circ} \mathrm{C}$ was also verified using VTPXRD (Fig. 29e), where ramping up or down the temperature from both extremes did not disrupt its framework integrity (Fig. 29f).

5.3.2 Single crystal X-ray diffraction (SCXRD). This is a very useful technique for structure determination in inorganic, organic and organometallic chemistry. It has been widely exploited for the structure determination of various discrete, inorganic polymeric compounds including metal-organic frameworks, ${ }^{185}$ but very less explored for crystalline organic polymers. The building mechanism of $2 \mathrm{D}$ and 3D COFs is highly complex and requires attentive efforts to obtain a suitable single crystal for this study. However, some success has been achieved in the case of three-dimensional frameworks, as mentioned in Section 3.2.2. In this section, we briefly discuss the use of the SCXRD technique for three examples.

In 2013, the first examples of single-crystalline azidoxylinked frameworks were reported, viz. NPN-1, NPN-2 and NPN-3, by Beaudoin et al. The crystals were grown in different solvents, yielding a good mosaic with a size of $\sim 10 \mathrm{~mm} .{ }^{88}$ Both NPN-1 and NPN-2 crystallized in the tetragonal space group $P \overline{4} b 2$, knitting four-fold-interpenetration along c-axis, whereas NPN-3 crystallized in the $P 4_{2} / n$ with six-fold-entanglement. All three frameworks exhibited the dia topology, in which the two $T_{\mathrm{d}}$-nodes are at a distance of 12.26, 12.76 and $15.39 \AA$ from each other for the methane, silane and adamantane-core tetranitroso unit. This is ascribed to the long C-Si bond in NPN-2 compared to the $\mathrm{C}-\mathrm{C}$ bond in NPN-1. Moreover, the adamantanecore is bigger, and thus the framework could afford six-fold interpenetration.

As mentioned earlier, the first imine-linked COF, COF-300, was analyzed by the PXRD technique and its simulated pattern to obtain its structural information in 2009. It was determined that the framework was crystallized in the tetragonal $I 4_{1} / a$ space group with the dia-c5, topology which does not obey the formula for the degree of interpenetration, i.e., $N=2 a / c$, where ' $a$ ' and ' $c$ ' are the cell parameters for the tetragonal lattice. Recently, it was found from the SCXRD data of the 3D COF-300 that the framework is seven-fold interpenetrated (dia-c7) with unit cell parameters of $a=b=28.280 \AA$ and $c=10.079 \AA$, which is in accordance with the formula (Fig. 31). ${ }^{6}$ The possible explanation for this is the aging effect, which increased the degree of interpenetration during its synthesis (vide supra, Section 3.2.2).

Besides COF-300, single crystals of the imine-linked 3D COF-303, LZU-79 and LZU-111 were also obtained and diffracted. It must be noted that the symmetry, topological view and degree of interpenetration in COF-300 and COF-303 are identical with a slight difference in imine connectivity, which could not be detectable using the PXRD technique. Therefore, the use of the SCXRD method is essential here.

The COF LZU-79 was reticulated from COF-300, in which the terephthaldehyde unit was replaced by the longer 4,7-bis(4-formylbenzyl)-1 $H$-benzimidazole (BFBZ). LZU-79 crystallizes in the $P 4_{2} / n$ space group with a high degree of entanglement (dia-c10). Moreover, large-sized crystals of a new chiral imine-linked LZU-111 COF with a size of $\sim 60 \mu \mathrm{m}$ were grown using aniline as the modulator. This chiral COF crystallizes in the $P 6_{5}$ space group and exhibits a different topology, i.e., lon-b-c3 (vide supra, Section 2.1)

For a single crystal to be successfully diffracted, the required size is $\sim 0.5 \mathrm{~mm}$ or larger. However, due to very little success in obtaining appropriately sized single-crystal COFs, the XRD technique has not been efficiently utilized in this area. Accordingly, the rotation electron diffraction method is employed when the single crystal size varies from nano- to micrometers. The RED technique is further discussed in Section 5.4.2.

\subsection{Microscopic techniques}

A very important and widely used characterization technique for the analysis of COFs is electron microscopy, which uses a negatively charged electron beam in place of a light source. 

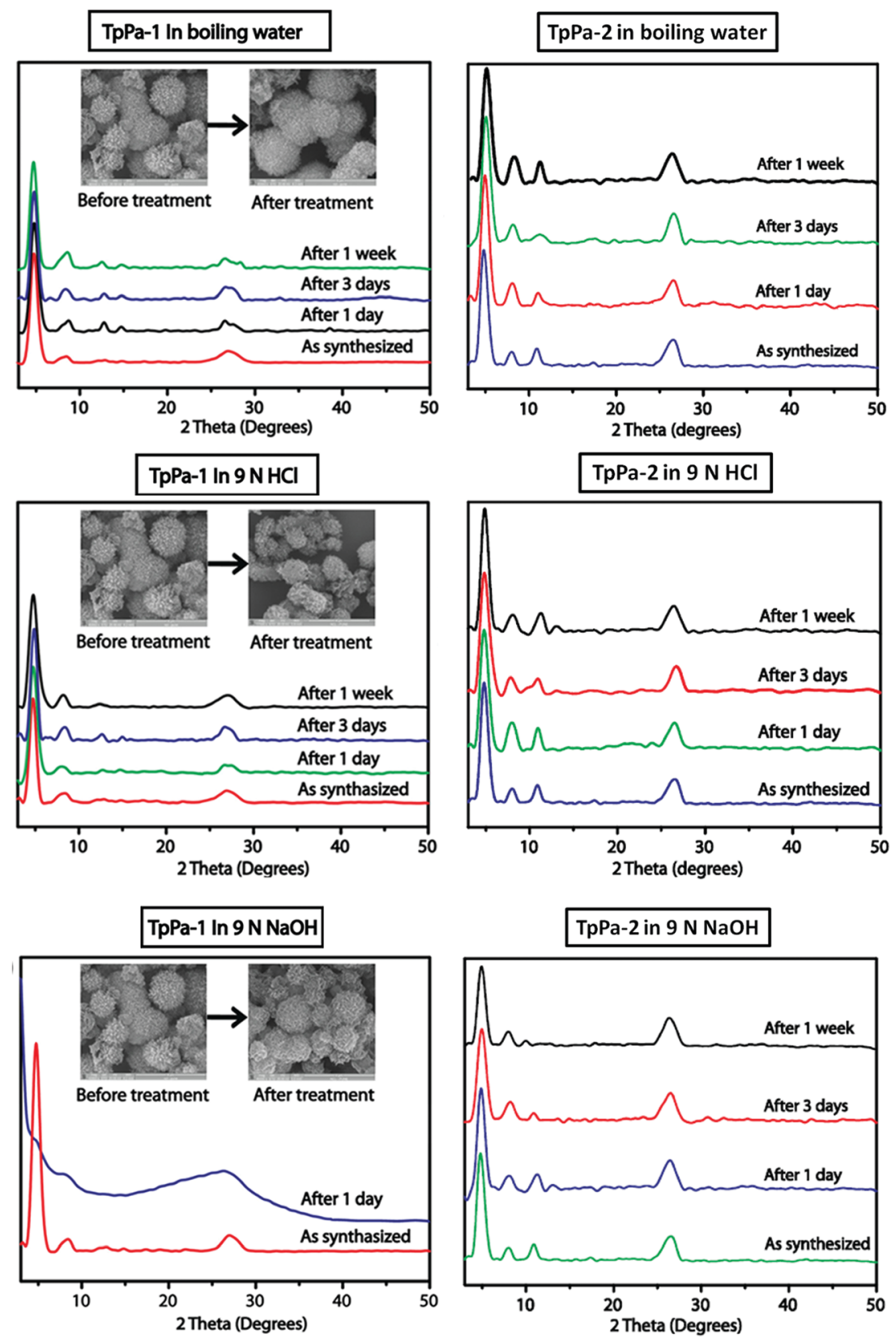

Fig. 30 PXRD patterns of TpPa-1 (left) and TpPa-2 (right) in boiling water, $9 \mathrm{~N} \mathrm{HCl}$ and $9 \mathrm{~N} \mathrm{NaOH}$, showing their stability. Reproduced with permission from ref. 67, Copyright the American Chemical Society (2012).

This technique gives detailed information about the properties of materials such as surface morphology, phase and bulk purity, reproducibility, and elemental mapping.

5.4.1 Field emission scanning electron microscopy (FESEM). This is a sophisticated microscopic technique, which aids in discovering the morphologies of COFs and analyzing the elements present in them. It also helps in determining the reproducibility and phase purity of samples. Almost all porous-organic frameworks are scanned by the FESEM technique and have been reported to possess distinct and eye-catching morphologies. The various surface structures (shown in Fig. 32) obtained for porous organic frameworks include spherical (a), ${ }^{186}$ hollow tubes (b), ${ }^{187}$ rods (c) ${ }^{141}$ flower like (d), ${ }^{68}$ fibre like (e), ${ }^{188}$ thin sheets/films (f) ${ }^{188}$ and flakes. Unique morphologies showing trepan-like $(\mathrm{g})^{138}$ (known as sea cucumber) and bouquet-like (h) ${ }^{189}$ structures have also been reported recently.

The homogeneity of the bulk sample and reaction completion can be confirmed using the FESEM technique, as shown in Fig. 32i. ${ }^{57}$ The first synthesized boroxine and boronic ester-linked COFs, COF-1 and COF-5, showed an aggregated platelet-like morphology (Fig. 33). ${ }^{4}$ The framework can have different particle sizes, varying from a few microns $(\mu \mathrm{m})$ to nanometers $(\mathrm{nm})$. Highly magnified electron images can be obtained using the TEM technique, as discussed in Section 5.4.2. 


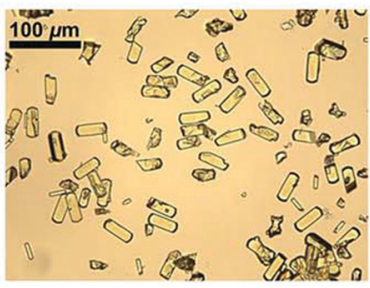

COF-300 $(\sim 60 \mu \mathrm{m})$

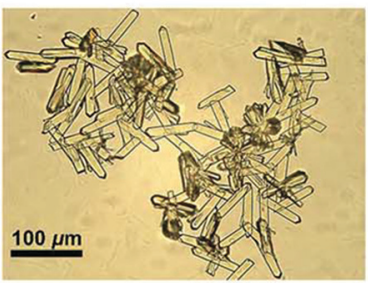

LZU-79 $(\sim 100 \mu \mathrm{m})$

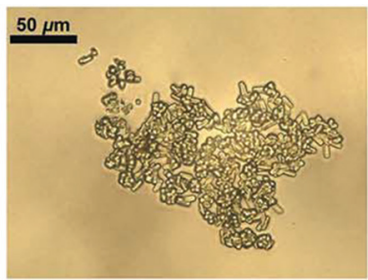

$\operatorname{COF}-303(\sim 15 \mu \mathrm{m})$

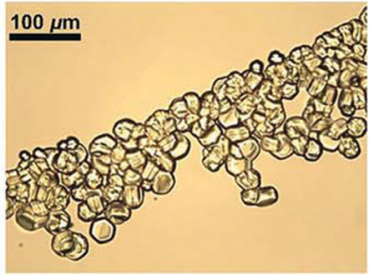

LZU-111 $(\sim 50 \mu \mathrm{m})$
Fig. 31 Optical microscopy images of single-crystalline COFs obtained in the presence of aniline. Reproduced with permission from ref. 6, Copyright The American Association for the Advancement of Science (2018).
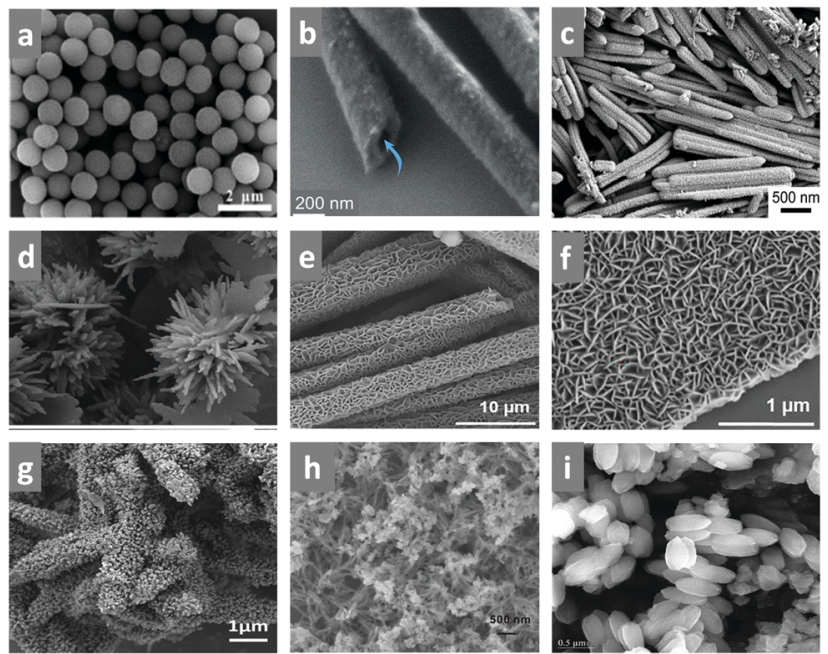

Fig. 32 FESEM images showing different surface morphologies of COFs Reproduced with permission from: (a) ref. 186, Copyright the American Chemical Society (2019); (b) ref. 187, Copyright John Wiley and Sons (2018); (c) ref. 141, Copyright the American Chemical Society (2019); (d) ref. 68, Copyright the American Chemical Society (2019); (e and f) ref. 188, Copyright Elsevier (2019). (g) ref. 138, Copyright The Royal Society of Chemistry (2021); (h) ref. 189, Copyright the American Chemical Society (2017) and (i) ref. 57, Copyright The American Association for the Advancement of Science (2020)

The stability of the framework is another factor that can be confirmed by checking the morphological stability of the material. In 2012, Banerjee and co-workers tested the chemical and water stability of two $\beta$-ketoenamine-linked frameworks, TpPa-1 and TpPa-2, using the FESEM method. ${ }^{67}$ As can be seen in the inset of Fig. 30 (left), there was no change in the morphology of TpPa-1. Both frameworks possess flower-like surface structures, which are possibly formed by aggregated gobs of petals. For TpPa-1, each petal is 1-3 $\mu \mathrm{m}$ long, 70-150 $\mathrm{nm}$ wide and 30-40 $\mathrm{nm}$ thick in size.
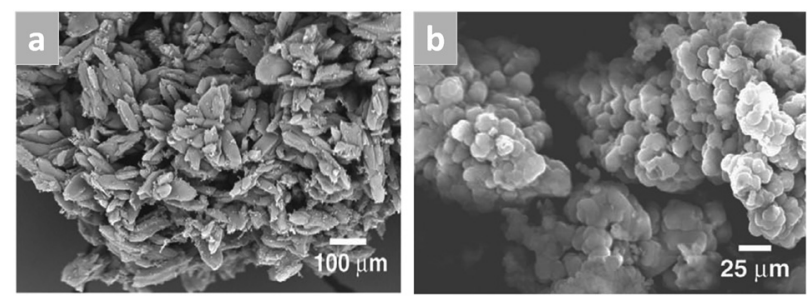

Fig. 33 FESEM images of bulk COF-1 (a) and COF-5 (b). Reproduced with permission from ref. 4, Copyright The American Association for the Advancement of Science (2005).

The petals are thicker and wider for TpPa-2. The electron images confirmed the stability of the $\mathrm{COF}$ in boiling water, $9 \mathrm{~N} \mathrm{HCl}$ and $9 \mathrm{~N} \mathrm{NaOH}$ for 7 days.

COFs exhibit sharp FESEM images, showing very interesting surface morphologies. Recently, this technique has been used to gain insight into the formation of different micro and nanostructures by conducting COF synthesis time-dependent studies. In 2019, the self-assembly of the hollow shuttle-shaped TPE-TAPCOF was studied at different intervals. ${ }^{190}$ The fully grown shuttlelike morphology of the COF was synthesized in 5 days; therefore, the generation of its microstructure was gradually studied after 1 , 2, 3, 4 and 5 day intervals (Fig. 34a). It can be observed from the FESEM images that after 1 day, a small nanospherical assembly was present, which further agglomerated into nanorods on $2 \mathrm{~d}$. These rods were about $100 \mathrm{~nm}$ in diameter and a few microns long. After the third day, the nanorods roughly assembled to yield a dense microcapsule. Further on days 4 and 5, the surface of the microcapsule became smoother and hollow cavities began to form inside the shuttle, which was confirmed by the TEM images (Fig. 34b). A pictorial representation of the self-assembly is depicted in Fig. 34c.

Moreover, the FESEM images of various microcrystals of NPN-1, NPN-2, NPN-3, COF-300, COF-303, LZU-59 and LZU-111 were measured, which revealed the formation of single-crystalline COFs. ${ }^{6,88}$

Energy-dispersive X-ray spectroscopy (known as EDX/EDS/ EDXS/XEDS) is another analytical technique that accompanies electron microscopy, which is widely used for elemental analysis of the sample. To verify the elements present in the covalentlybonded organic porous framework, chemical characterization is performed, which mostly gives the electronic image with the \% of elements present in it and their mapping. An example of this is $\mathrm{COF}-\mathrm{SO}_{3} \mathrm{H}$ and $\left[\mathrm{NH}_{4}\right]^{+}\left[\mathrm{COF}-\mathrm{SO}_{3}{ }^{-}\right]$, where their SEM images and EDX mapping are shown in Fig. 34d. ${ }^{191}$

A few years ago, the effect of different synthetic methods on the surface morphology of the framework was studied. Banerjee and co-workers reported three distinct morphologies for the TpPa-1 COF under three different synthetic conditions. ${ }^{67,83,84}$ The dark-red COF possessed a flower-like, graphene-sheet like and ball-like surface when synthesized under solvothermal, mechanochemical and microwave irradiation conditions (Fig. 35). The flower-shaped morphology resulted from the aggregation of the sheet-like petals, which were 70-150 nm wide and 30-40 nm thick. The time and conditions required to form this distinct 
a

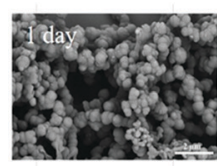

b

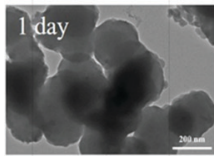

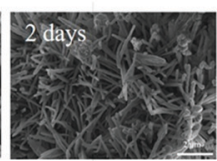
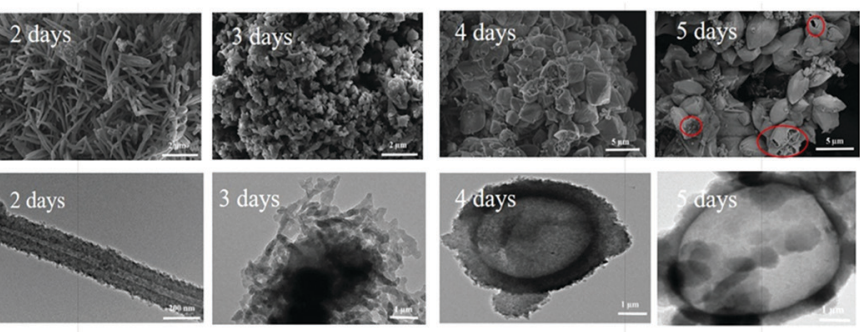

C

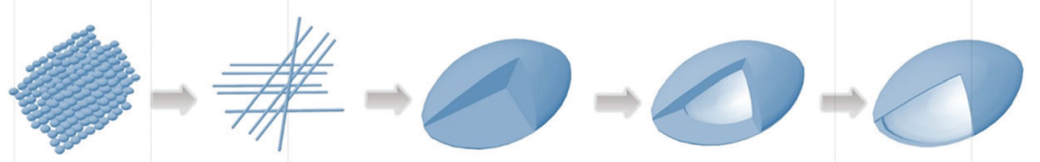

d
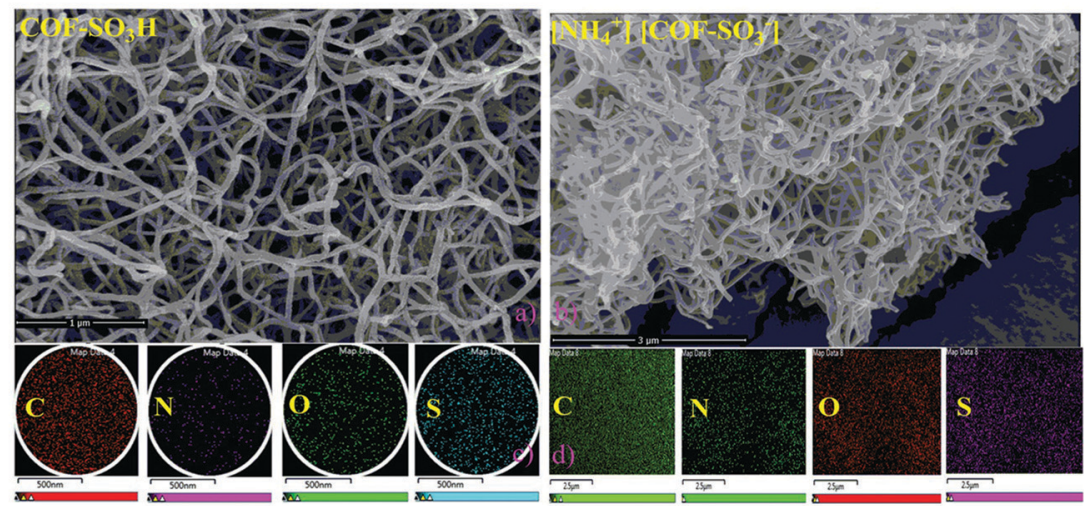

Fig. 34 (a) FESEM and (b) TEM images of TPE-TAP-COF recorded at different intervals. (c) Mechanism for the formation of COF hollow microshuttle. (d) FESEM images (top) and EDS mapping (bottom) results of COF-SO ${ }_{3} \mathrm{H}$ (left) and $\left[\mathrm{NH}_{4}\right]^{+}\left[\mathrm{COF}-\mathrm{SO}_{3}{ }^{-}\right]$(right). Reproduced with permission from: (a-c) ref. 190, Copyright The Royal Society of Chemistry (2019) and (d) ref. 191, Copyright 2019 Creative Commons CC by license.

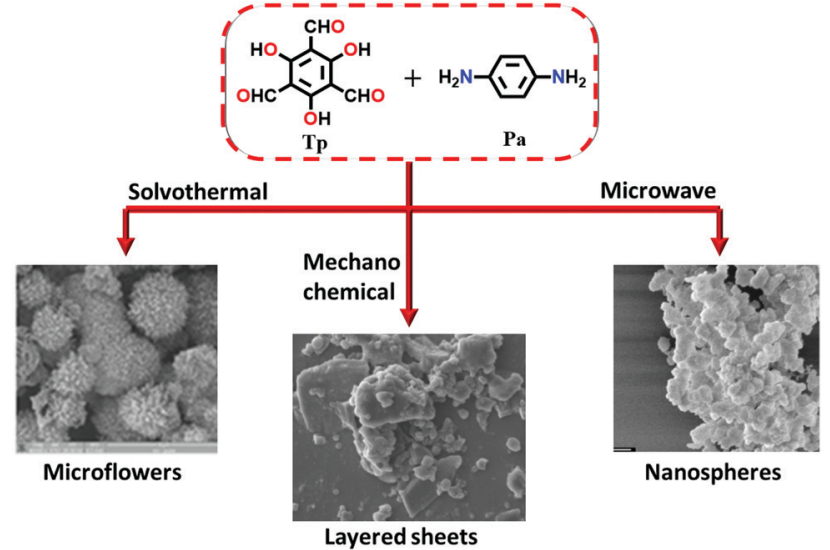

Fig. 35 Pictorial representation of TpPa-1 COF synthesized using triformylphloroglucinol $(\mathrm{Tp})$ and phenylenediamine $(\mathrm{Pa})$, acquiring three different morphologies under different reaction conditions. Reproduced and adapted from ref. 67, Copyright the American Chemical Society (2012); ref. 83, Copyright the American Chemical Society (2013) and ref. 84, Copyright The Royal Society of Chemistry (2015).

morphology were provided via the thermodynamically supported solvothermal method. However, when the framework was constructed by constant grinding, the 2D sheets tended to delaminate by breaking the supramolecular stacking forces. This can be seen when graphene-like layered sheets were formed instead of a flower. For the ball-like morphology with a rough surface, the diameter of each ball was $\sim 30 \mathrm{~nm}$, which is consistent with the thickness of the flower petals formed during the solvothermal synthesis.

Similarly, Shinde et al. observed that two distinct surface patterns in TpBpy COF existed when the Tp and Bpy monomers were subjected to (a) solvothermal $\left(120{ }^{\circ} \mathrm{C}, 72 \mathrm{~h}\right)$ and (b) mechanochemical (30 Hz, $90 \mathrm{~min})$ conditions, respectively. ${ }^{192}$ In the high temperature vacuum environment, the small COF crystallites (100-300 $\mathrm{nm}$ wide) agglomerated to form fine threadlike shapes, which interweave. Conversely, the framework obtained via the mechanochemical approach formed sheets of smaller dimensions due to exfoliation by constant grinding.

5.4.2 Transmission electron microscopy (TEM). This is a highly exploited electron microscopic technique, which helps in determining the morphology of COFs on the nanometer scale, very close to the atomic level. The main difference between the TEM and FESEM techniques is that the latter scans image by detecting the electrons reflected from the sample surface, whereas the former utilizes the transmitted electrons, which pass through the sample to give a highly magnified image. Moreover, high-resolution TEM images (below $20 \mathrm{~nm}$ scale) can be obtained from this technique, which give very detailed and highly magnified surface structures. For example, various COFs with HRTEM images of different morphologies, nanospheres (a) ${ }^{186}$ hollow tubes (b and c),${ }^{193}$ nanofilm (d), ${ }^{188}$ trepan like (e) ${ }^{138}$ and bouquet like $(\mathrm{f})^{189}$ are shown in Fig. 36. 

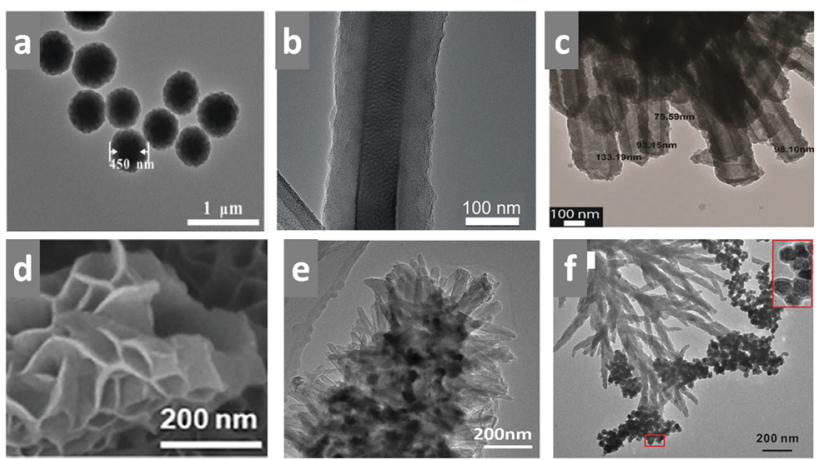

Fig. 36 TEM images showing different surface morphologies. Reproduced with permission from: (a) ref. 186, Copyright the American Chemical Society (2019); (b) ref. 187, Copyright John Wiley and Sons (2018); (c) ref. 193, Copyright The Royal Society of Chemistry (2015); (d) ref. 188, Copyright Elsevier (2019); (e) ref. 138, Copyright The Royal Society of Chemistry (2021) and (f) ref. 189, Copyright the American Chemical Society (2017).

A distinctive surface morphology fabricated from a flowerlike surface via the self-assembly of nanosheets was reported by Das et al. in 2019. The bis(imidazole)-linked TBICOF, which was solvothermally synthesized (vide supra), sets in a uniformly ordered porous hexagonal arrangement, which could be observed by HRTEM (Fig. 37). ${ }^{68}$ During the course of the 3 day reaction, the nanosheets combine, forming a ring-like assembly, and set in a microfloral morphology with an average diameter of 150-200 nm and height of 12-15 $\mathrm{nm}$. STEM (scanning transmission electron microscopy) images of the microflower surface were also taken, in which the electron beam is focussed at a wider angle and scans through the sample.

Previously, it was mentioned that acquiring crystallinity in a framework endowed with stability and functionalization is very complex. However, this technique helps to monitor the
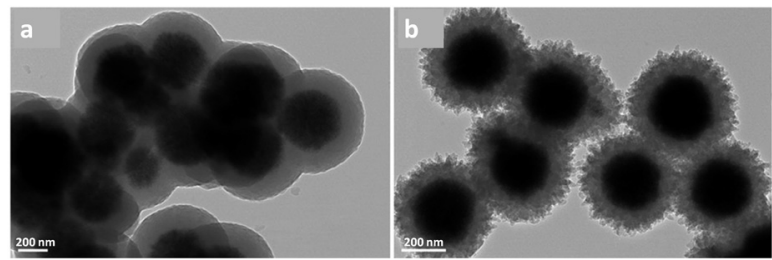

Fig. $38 \mathrm{HR}$-TEM images of $\mathrm{Fe}_{3} \mathrm{O}_{4}$ (apolyimine (a) and $\mathrm{Fe}_{3} \mathrm{O}_{4}(\mathrm{aCOF}(\mathrm{TpBD})$ (b). Reproduced with permission from ref. 194, Copyright John Wiley and Sons (2016).

amorphous and crystalline nature by scanning in-depth electron images of the framework. Wang and co-workers reported an example of this, in which the amorphous-to-crystalline transformation of an $\mathrm{Fe}_{3} \mathrm{O}_{4}$-templated polyimine COF (a) into $\mathrm{Fe}_{3} \mathrm{O}_{4} @ \mathrm{COF}(\mathrm{TpBD})$ (b) was carried out solvothermally. The HRTEM images were taken to record the reorganization of the disordered framework into well-defined and ordered microspheres (Fig. 38). ${ }^{194}$

The growth of microstructures from nanoparticles can be well studied by TEM and HRTEM images. An example of this is shown is Section 5.4.1, Fig. 34b. Recently, the formation of hollow tubes of the TPT-DAHQ COF with sponge-like shells, which occurred via aggregation, self-assembly and Ostwald ripening of the COF, was examined (Fig. 39 ). ${ }^{164}$ The solvothermally synthesized COF (see Section 4.2) investigated via a timedependent study, where the reaction was quenched after 12 , 24 and $48 \mathrm{~h}$ to obtain the high-resolution image. It was observed that after $12 \mathrm{~h}$, very small ribbon-like nanocrystallites of about $40-50 \mathrm{~nm}$ width aggregated, which correspond to the $\pi-\pi$ stacked 2D COF sheets. Maintaining high-temperature conditions for $24 \mathrm{~h}$, the minute planar crystallites assembles to form dense tubules whose walls were formed by the aggregation of the ribbon-like nanocrystallites. After $48 \mathrm{~h}$, an exceptional
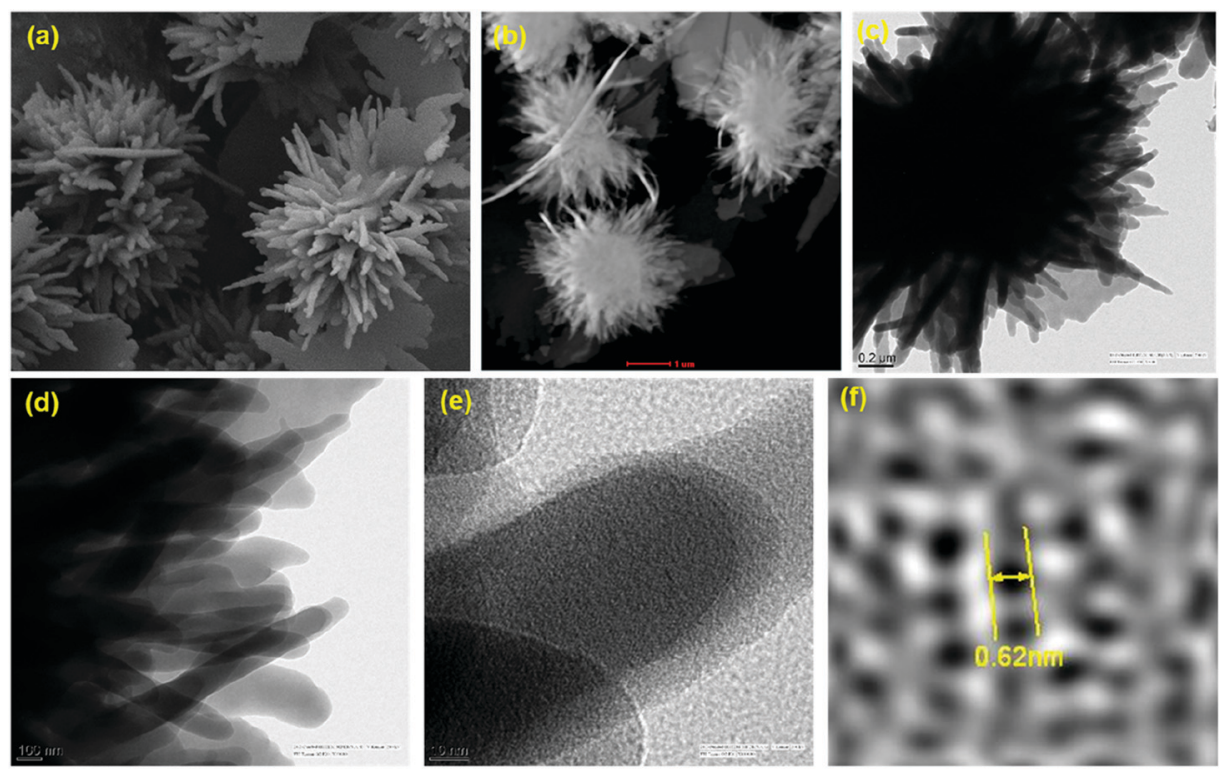

Fig. 37 (a) FESEM image focusing on the microflower areas in TBICOF; (b) STEM image of TBICOF; (c) TEM image of TBICOF depicting microflowers; and (d-f) HRTEM images of TBICOF. Reproduced with permission from ref. 68, Copyright the American Chemical Society (2019). 
a

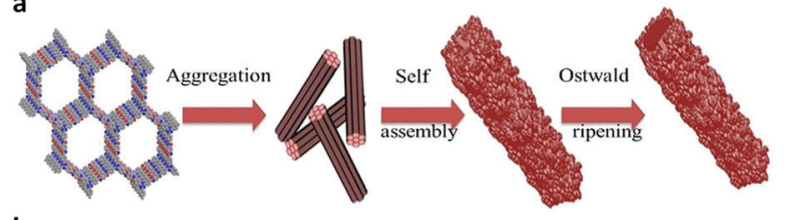

b

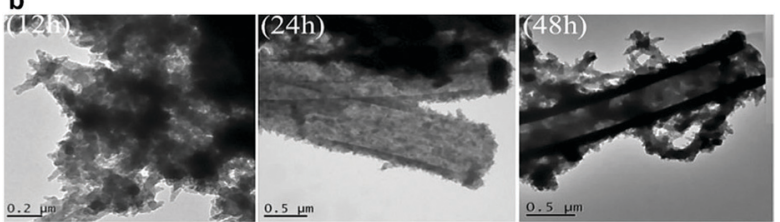

Fig. 39 (a) Expected mechanism for the formation of the hollow microtubular TPT-DAHQ COF having sponge-like shells. (b) TEM images of the hollow microtubular TPT-DAHQ COF having sponge-like shells, measured after reaction times of 12,24 , and $48 \mathrm{~h}$. Reproduced with permission from ref. 164, Copyright John Wiley and Sons (2019).

phenomenon of the Ostwald ripening process occurred, by which the crystallites present inside the hollow tubules migrated towards the outer surface to reduce their surface energy. This destroyed the tubules, and hollow microtubes were formed with rough crystallites located on their surface. At the end of the reaction after $72 \mathrm{~h}$, the ribbon-like crystallites present on the outer surface of the microtubules displays a smooth sponge-like surface. The resulting tubules had an average length of $7.4 \mu \mathrm{m}$ and average diameter of $1 \mu \mathrm{m}$. The average values for the outer and inner diameters of the tubules were 0.90 and $0.53 \mu \mathrm{m}$, respectively.

In addition to images, the bulk phase purity of powdered frameworks has also been studied using selected area electron diffraction (SAED). This technique is beneficial to observe the diffraction spots, and thus gives information about the order of the framework. Moreover, using fast Fourier transformation (FFT), the d-spacing between the two planes can be closely measured. Consequently, the high and low-angle grain boundaries can be identified, providing that the framework has exceptionally high crystallinity, stability and rigidity. This type of study was performed by Lotsch and co-workers on TTT-COF and TTI-COF. ${ }^{170}$ Besides, phenomena such as twinning, or some framework defects can also be detected.

It is well known that TEM usually produces flat 2D images, which lack some features compared to FESEM, given that a 3D image of the sample can be obtained. However, a crucial study using 3D diffraction tomography (3D-EDT) can also be conducted using the TEM technique (Fig. 40). 3D-EDT is basically an extension of the regular TEM, in which the sample is rotated or tilted at different angles around its center to obtain a threedimensional image. An example of this was reported in 2016 by Yaghi and co-workers using a rare woven COF, COF-505. ${ }^{195}$

The FESEM and TEM images showed that spheres with a diameter of $2 \mathrm{~mm}$ were formed through the agglomeration of small crystallites with a size of $\sim 200 \mathrm{~nm}$. The 3D-EDT data was obtained by tilting the sample and the electron beam in the range of $-41.3^{\circ}$ to $+69.1^{\circ}$ with a beam-tilt step of $0.2^{\circ}$. According to the acquired tomography data, the C-centred orthorhombic

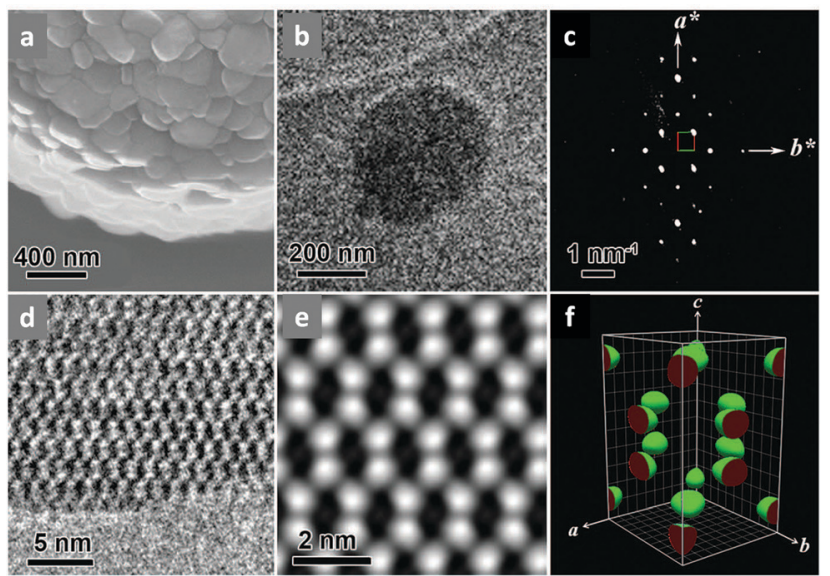

Fig. 40 Morphology and electron microscopy studies of COF-505. (a) Crystallites aggregated on a crystalline sphere observed by SEM. (b) TEM image of a single sub-mm crystal used for 3D-EDT. (c) 2D projection of the reconstructed reciprocal lattice of COF-505 obtained at $298 \mathrm{~K}$ from a set of 3D-EDTdata. (d) HRTEM image of COF-505 taken with the [1-10] incidence. (e) 2D projected potential map obtained by imposing pgg plane group symmetry. (f) Reconstructed 3D electrostatic potential map (threshold, 0.8). Reproduced with permission from ref. 195, Copyright The American Association for the Advancement of Science (2016).

lattice was constructed with cell parameters of $a=18.9 \AA$, $b=21.3 \AA, c=30.8 \AA$, and $V=12399 \AA^{3}$.

A rarely used technique for the structure determination of COFs using the TEM analytical method is $3 \mathrm{D}$ rotation electron diffraction (RED). In this technique, the fairly small-sized single-crystalline imine-linked COF-320 was evenly spread on a carbon-coated $\mathrm{Cu}$ grid via the drop-casting method. Thereafter, the 3D-RED data was collected in SAED mode at $298 \mathrm{~K}$ with the diameter of the chosen aperture greater than the crystal size. The ED images were taken by tilting the crystal base in the range of $-35.00^{\circ}$ to $+25.10^{\circ}$ with a beam-tilt step of $0.4^{\circ}$. The data was processed and detailed structural information was obtained, indicating the tetragonal $I \overline{4} 2 d$ space group with the dia-c9 topology (Fig. 41a). ${ }^{89}$ The image of the reconstructed reciprocal lattice from 3D-RED data is shown in Fig. 41b-d.

5.4.3 Atomic force microscopy (AFM). Besides the aforementioned microscopic methods, AFM is used to obtain a more precise three-dimensional profile of the sample to clearly view its surface roughness and height. AFM scans the image of a micrometer square area with a depth of field of 10-20 $\mu \mathrm{m}$. COFs exist in the form of 2D-layers stacked on each other or in the interpenetrated 3D form (vide supra). Therefore, the heightprofile image is essential to check the number of layers stacked. One example is the hcc-COF thin film, where the AFM image showed the stacking of nine layers with a height of $\sim 3.8 \mathrm{~nm}$ (Fig. 42a). ${ }^{163}$ Likewise, the AFM height-profile image revealed the rough surface of the $\mathrm{COF}_{\mathrm{DAAQ}-\mathrm{BTA}-3 \mathrm{DG}}$ composite in comparison to graphene, with the average size 50-100 nm (Fig. 42b and c), respectively. ${ }^{183}$ Bare graphene is nearly flat and featureless when observed via microscopy. Fig. 42d depicts the scratched surface of the COF $_{\text {DAAQ-BTA-3DG }}$ film, showing a thickness of $\sim 185 \mathrm{~nm}$. Fig. 42e and $\mathrm{f}$ show the AFM images and the height profiles 
a
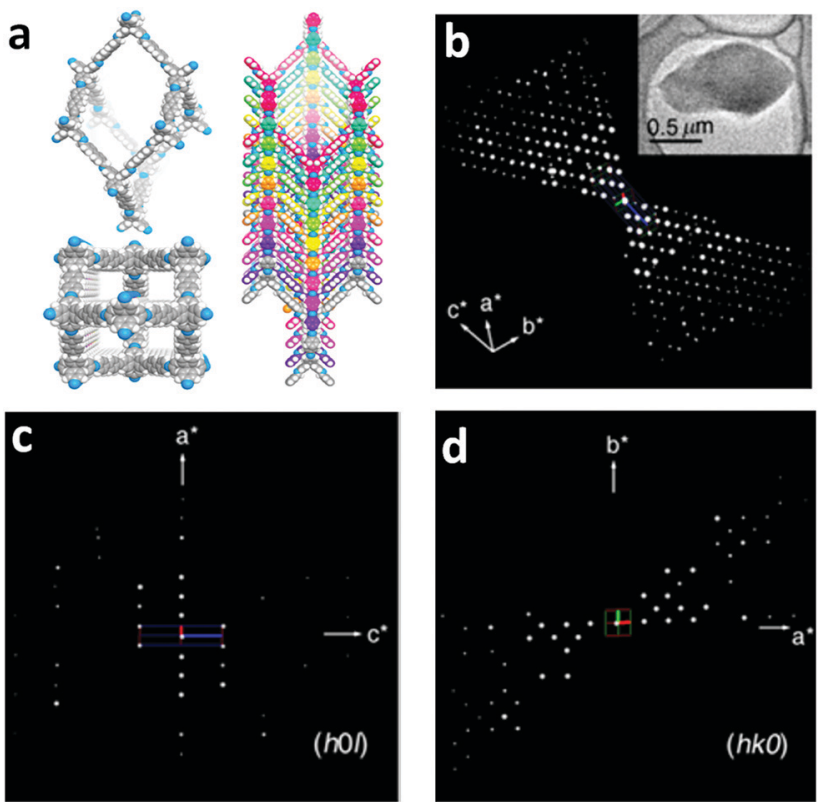

Fig. 41 Morphology and electron diffraction of COF-320. (a) SEM image of the aggregation of crystallites. (b) 3D reciprocal lattice of COF-320 reconstructed from the RED data collected from a crystal of $1.0 \times 0.5 \times$ $0.2 \mu \mathrm{m}^{3}$ (inset). (c) $(h 0 l)$ and (d) $(h k 0)$ slices cut from the reconstructed reciprocal lattice. Reproduced with permission from ref. 89, Copyright the American Chemical Society (2013).

of two covalent organic nanosheets, namely TpBDH and TfpBDH CONs, which were efficiently used as turn-on sensors (vide infra). ${ }^{149}$

\subsection{X-ray photoelectron spectroscopy (XPS)}

This technique is employed to determine the composition of elements, which helps in characterizing the bulk structure of materials. COFs are composed of light elements, such as B, C, $\mathrm{N}, \mathrm{O}, \mathrm{S}, \mathrm{P}$ and Si, and thus the peaks in an XPS spectrum, which gives a plot of the number of electrons vs binding energy, directly indicate the identity and amount of an element present in the framework. The XPS peaks also display the subshell (1s, $2 \mathrm{~s}, 2 \mathrm{p}, 3 \mathrm{~s}$, etc.) from which the electron is ejected when the $\mathrm{X}$-ray beam falls on it. In addition, the bonding between the two monomer units can also be determined using this spectroscopic technique. A few $\mathrm{N}$-rich frameworks characterized by this method are discussed below.

Triazine-linked frameworks. The CTF-1 framework contains only $\mathrm{C}$ and $\mathrm{N}$-atoms, for which the XPS spectra show two peaks each for C1s and N1s nuclei (Fig. 43). ${ }^{120}$ The C1s element exhibits two peaks having binding energy values of $284.6 \mathrm{eV}$ and $286.2 \mathrm{eV}$, which are assigned to aromatic $\mathrm{sp}^{2}$-carbon and the triazine-ring carbon, respectively. Conversely, the N1s XPS spectrum displays two signals with binding energy values of $398.8 \mathrm{eV}$ and $400.2 \mathrm{eV}$, respectively, where the former is assigned to the nitrogen of the triazine core and the latter to the $\mathrm{N}$-atom from the nitrile moiety that remained in the uncondensed form. Relative to CTF-1, the CTF-1-600 framework shows a similar XPS spectrum with a small contribution
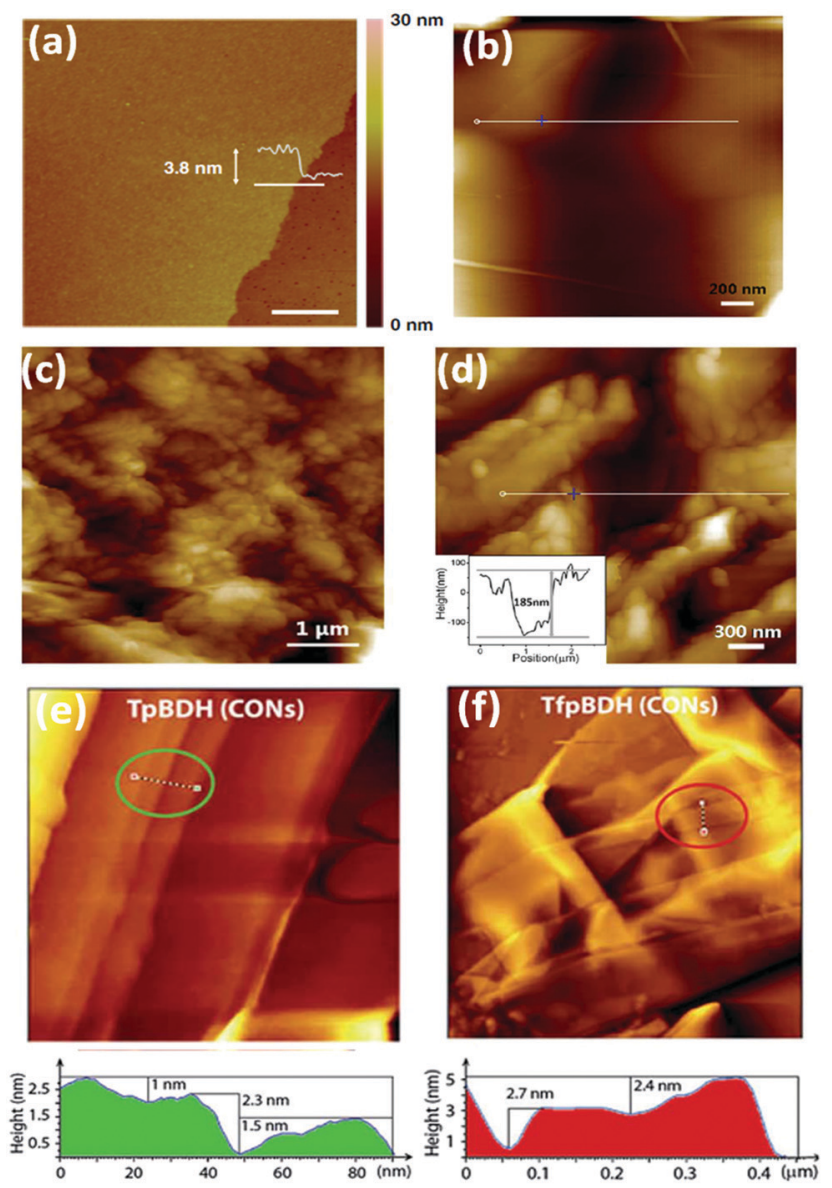

Fig. 42 AFM image and height profile of (a) hcc-COF film (scale bar = $1 \mu \mathrm{m})$, (b and c) COF DAAQ-BTA-3DG composite, (d) scratched COF DAAQ-BTA-3DG composite and the corresponding section profile, (e) TfpBDH-CONs and (f) $\mathrm{TpBDH}-\mathrm{CONs}$ with the corresponding height profiles below (the height profiles filled in red and green colour were measured along the corresponding tracks inside the circle shown in the AFM images). Reproduced and adapted with permission from: (a) ref. 163, Copyright 2019 reprinted under the terms of Creative Commons Attribution 4.0 International license; (b-d) ref. 183, Copyright the American Chemical Society (2015) and (e and f) ref. 149, Copyright The Royal Society of Chemistry (2015).

due to $\mathrm{C} 1 \mathrm{~s}$ at $(288.0 \mathrm{eV})$ of low intensity. This peak corresponds to the carbon-atom from the - $\mathrm{COO}$ group, which is observed due to the partial oxidation and dissociation of the framework at high temperature. Moreover, the intensity of the XPS peaks in the N1s spectrum for CTF-1-600 was observed to be high for the nitrile $\mathrm{N}$-atom from the cyano group.

By introducing electron-withdrawing fluorine atoms in the CTF-1 moiety, the degree of polymerization was reduced. This was confirmed by the XPS spectra of FCTF-1 (fluorinated CTF-1), where the peak intensity of the uncondensed $\mathrm{N}$-atom at $400.5 \mathrm{eV}$ increased significantly in comparison to that at $398.8 \mathrm{eV}$, which was originally assigned to the triazine nitrogen. Furthermore, the chemical nature of the $\mathrm{C}$ and $\mathrm{F}$ atoms was also explained using this technique given that the C1s spectrum can be deconvoluted into four peaks as follows: two usual peaks at $284.6 \mathrm{eV}$ (for aromatic $\mathrm{sp}^{2}$-C) and $286.2 \mathrm{eV}$ (triazinering carbon) and two arising from F-substitution at $287.8 \mathrm{eV}$ 
a

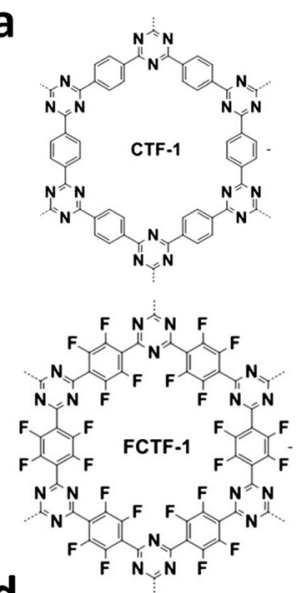

d

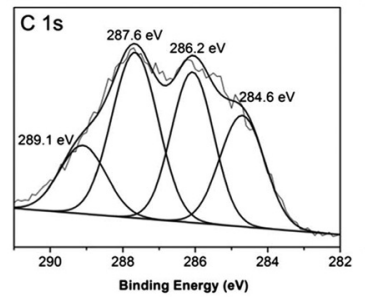

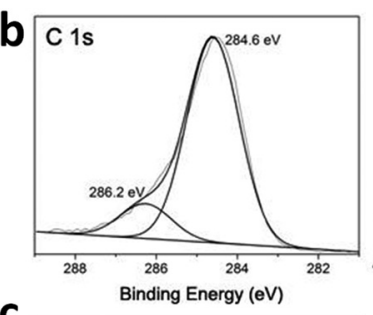
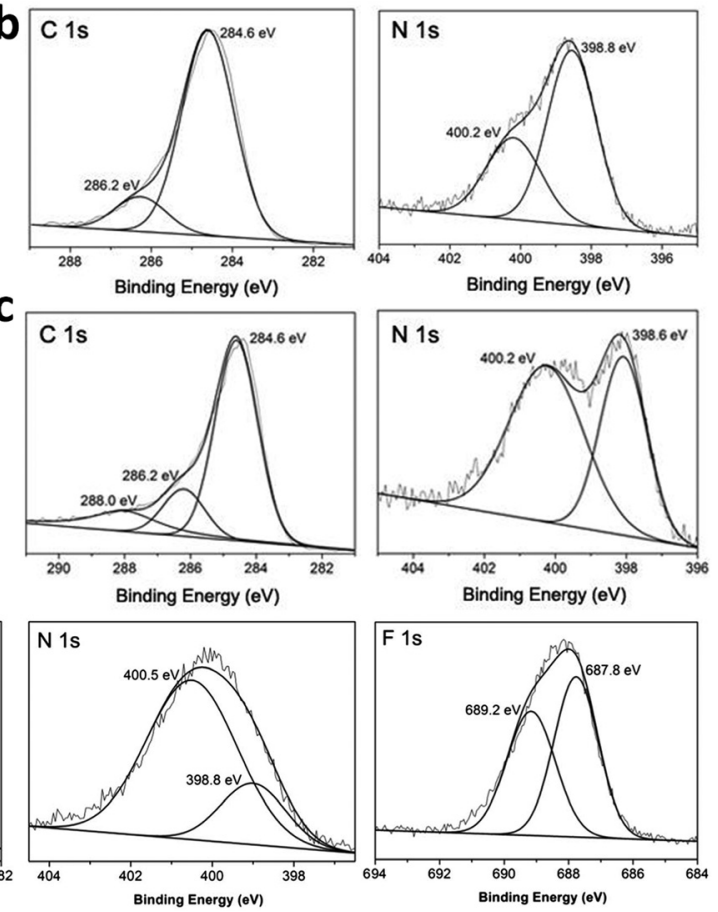

Fig. 43 (a) Structures of CTF-1 and FCTF-1. (b-d) C1s, N1s and F1s XPS spectra of triazine-linked COFs, CTF-1, CTF-1-600 and FCTF-1, respectively. Reproduced with permission from ref. 120, Copyright The Royal Society of Chemistry (2013).

(semi-ionic C-F bond) and $289.1 \mathrm{eV}$ (covalent C-F bond). In the F1s spectrum, two signals assigned to partial-ionic $(687.8 \mathrm{eV})$ and covalent C-F bonds $(689.2 \mathrm{eV})$ were observed. In the N1s XPS spectrum, FCTF-1 COF displays more striking peaks of nitrile in comparison to CTF-1, which clearly indicates that the fluorine-substituted precursor reduces the degree of polymerization in the framework.

Later, in 2015, the conversion of the triazine-linked framework from a lower $m$-CTF net to higher dimensional PTF net was reported, and the transformation was witnessed by the deconvoluted XPS N1s spectral peaks. ${ }^{121}$ The $m$-CTF net displays the two basic C1s peaks and one N1s peak at $\sim 284.4, \sim 286.3$ and $\sim 398 \mathrm{eV}$, respectively, where the latter peak suggests the existence of only one type of pyridyl nitrogen in the triazine ring. Conversely, the 3D porous triazine framework, namely PTF, that was synthesized at an elevated temperature showed two extra peaks in the N1s XPS spectrum, which were assigned to pyrrolic nitrogen at $\sim 399.5 \mathrm{eV}$ and quaternary nitrogen at $\sim 400.8 \mathrm{eV}$.

Imine-linked frameworks. In the XPS spectra of frameworks containing imine linkages, two major peaks can be observed, one near $285 \mathrm{eV}$, corresponding to the imine-carbon in the $\mathrm{C} 1 \mathrm{~s}$ spectrum, and the other for its N-atom near $398 \mathrm{eV}$ in the N1s spectrum. This method supports the formation of imine-bonded frameworks using the Schiff base condensation reaction. However, sometimes an additional peak for the unreacted amine monomer can be seen in the N1s XPS spectrum. An example of this is SCOFLZU-1, which was synthesized via the interfacial method using a combination of a trialdehyde and diamine.

The XPS spectra of SCOF-LZU-1 depict the standard peaks for the carbon and nitrogen atoms of the imine bond in the C1s and N1s spectra at 285.1 and $398.6 \mathrm{eV}$, respectively (Fig. 44a). In addition, an extra peak in both the spectra was observed at $284.4 \mathrm{eV}$ for the SCOF backbone and HOPG substrate and at $399.7 \mathrm{eV}$ for the nitrogen from the unreacted diamine unit. ${ }^{101}$

Hydrazone-linked frameworks. The highly luminescent BthDma COF contains free $\mathrm{O}-$ and $\mathrm{N}$-donor atoms, which provide free chelating sites for binding metal ions (vide supra). The selective binding of $\mathrm{Fe}(\mathrm{III})$ ions in these sites was monitored using the N1s spectrum (Fig. 44b). A shift in the binding energy value from 400.1 to $400.6 \mathrm{eV}$ indicates of $\mathrm{Fe}^{3+}$ binding in the chelating site. Moreover, the Fe2p spectrum could be deconvoluted into two peaks at 711.4 and $724.8 \mathrm{eV}$, which are attributed to the $\mathrm{Fe} 2 \mathrm{p}_{3 / 2}$ and $\mathrm{Fe} 2 \mathrm{p}_{1 / 2}$ binding energies, respectively, confirming the binding Fe(III) ions in the Bth-Dma COF system. ${ }^{141}$

This year, a similar example was reported, where XPS measurement verified the $\mathrm{Fe}(\mathrm{III})$ ion sensing by the Tfpa-Mth COF, forming Tfpa-Mth COF@Fe ${ }^{3+} \cdot{ }^{134}$ Before the sensing experiment, the Tfpa-Mth COF exhibited two peaks at 401.17 and $400.17 \mathrm{eV}$ for $\mathrm{C}-\mathrm{N}$ (unreacted Tfpa and $\mathrm{Mth}$ ) and $\mathrm{C}=\mathrm{N}$ (imine bond), respectively, in its N1s XPS spectrum. There was a decrease in the binding energy values after binding to the $\mathrm{Fe}^{3+}$ ions (400.88 and $399.90 \mathrm{eV}$ ), suggesting the successful fixation of $\mathrm{Fe}^{3+}$ ions to the wall of the pores. Moreover, the O1s XPS spectrum of the COFs also showed the BE values of 531.40 and $531.11 \mathrm{eV}$ before and after the sensing experiment, respectively, which support the detection of Fe ions. In the Fe2p spectrum, the characteristic signals at 712.01 and $725.07 \mathrm{eV}$ were assigned to the binding energies of $\mathrm{Fe} 2 \mathrm{p}_{3 / 2}$ and $\mathrm{Fe} 2 \mathrm{p}_{1 / 2}$, respectively.

For COF-DB and COF-DT, three intense peaks were observed in their C1s, O1s and N1s spectra, which were comprised of 
a SCOF-LZU-1

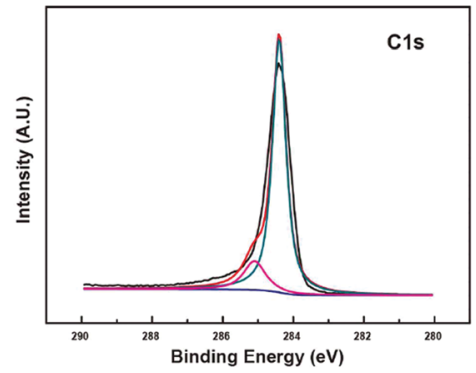

b Bth-Dma and Bth-Dma@Fe ${ }^{3+}$

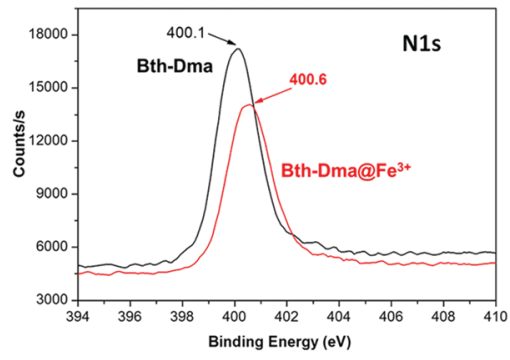

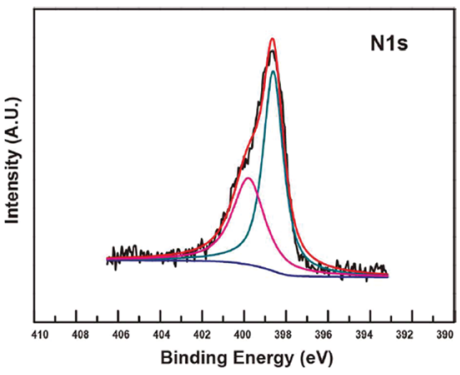

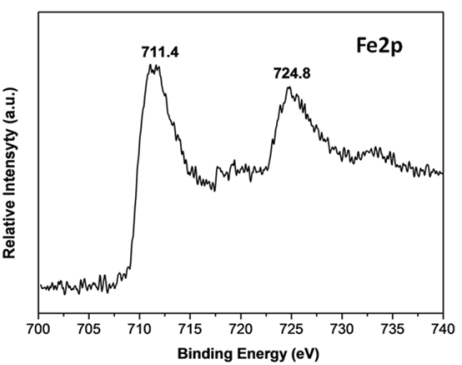

Fig. 44 (a) C1s and N1s XPS spectra of imine-linked SCOF-LZU-1. (b) N1s and Fe2p XPS spectra of hydrazone-linked Bth-Dma and Bth-Dma@Fe ${ }^{3+}$ COFs. Reproduced with permission from: (a) ref. 101, Copyright the American Chemical Society (2013) and (b) ref. 141, Copyright the American Chemical Society (2019)

multiple signals depicted from the high-resolution XPS spectra. It was observed that C1s and O1s show similar binding energy values for both COFs. Conversely, the N1s spectra showed peaks at 399.2 and $398.4 \mathrm{eV}$, which indicate the presence of a $\mathrm{C}-\mathrm{N}=\mathrm{C}$ center corresponding to the picoline and the triazinecores for COF-DB and COF-DT, respectively. ${ }^{131}$

Olefinic-linked frameworks. The formation of a $\mathrm{Csp}^{2}-\mathrm{Csp}^{2}$ bonding linkage using various organic reactions was well explored and discussed in the previous sections. Its characterization to obtain structural information was supported by XPS. For example, the 2DPPV COF was formed via the Knoevenagel condensation and its formed structure was compared to its monomers to validate its synthesis. The C1s XPS spectrum depicts two peaks at around $284.5 \pm 0.1$ and $286.0 \pm 0.5 \mathrm{eV}$, which are ascribed to the $\mathrm{sp}^{2}$-hybridised aromatic carbon and the substituted cyano carbon, respectively. ${ }^{158}$ Additionally, the N1s spectrum could be deconvoluted, exhibiting the binding energy at $398.0 \pm 0.1 \mathrm{eV}$ for the nitrogen of the cyano group (Fig. 45a and b).
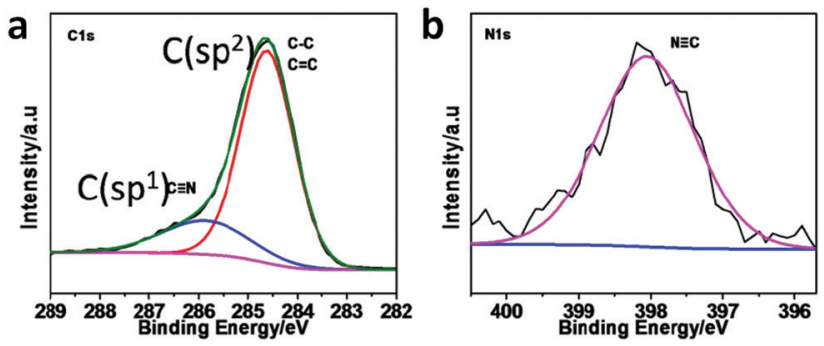

Fig. 45 (a) C1s and (b) N1s XPS spectra of 2DPPV COF. Reproduced with permission from ref. 158, Copyright The Royal Society of Chemistry (2016).
Other linkages. For the aminal-linkage in aminal-COF-1 and aminal COF-2, the strong peaks for C1s and N1s near 398 and $284 \mathrm{eV}$ are attributed to their framework, which is rich in C and $\mathrm{N}$ atoms, whereas the very low intensity of O1s just above $500 \mathrm{eV}$ proves that almost negligible aldehydic monomer was left. ${ }^{38}$

To gain insight into the chemical structure of the metal ions in the dioxin-linked Ni/Co/ZnPc-TFPN COF (Fig. 46), ${ }^{171}$ the XPS of NiPc-TFPN COF was considered. It showed peaks at $855.7 \mathrm{eV}$ for $\mathrm{Ni}_{2} \mathrm{p}_{3 / 2}$ and $873.0 \mathrm{eV}$ for Ni2 $\mathrm{p}_{1 / 2}$ in the Ni2p XPS spectrum. This corresponds to the +2 oxidation state of Ni. Similarly, the Co2p and Zn2p XPS spectra are also indicative of divalent metal ions.

\subsection{Gas sorption}

Porous frameworks have been known for a long time and proven to be potent candidates for a number of applications. Covalently bonded organic frameworks exhibit large surface areas with multiple types of micro-, meso- and macropores, which are tailored by maintaining their topology (vide supra). The surface area, pore volume and pore size of these porous materials can be calculated from the gas adsorption-desorption isotherms using the multi-layered Brunauer-Emmett-Teller (BET) adsorption method. There are six different types of adsorption isotherms, as shown in Fig. $47,{ }^{196}$ which are classified as type I to VI and reveal information about the nature of sorption, type of pores depending on their size, adsorption capacity, etc. The isotherms are obtained via the physical adsorption of inert and non-polar gases $\left(\mathrm{N}_{2}\right.$ and $\left.\mathrm{Ar}\right)$ in the pores of the material. The type I isotherm is specifically exhibited by microporous materials (pore size $<2 \mathrm{~nm}$ ), indicating limited gas uptake due to monolayer adsorption. It is specifically 
a
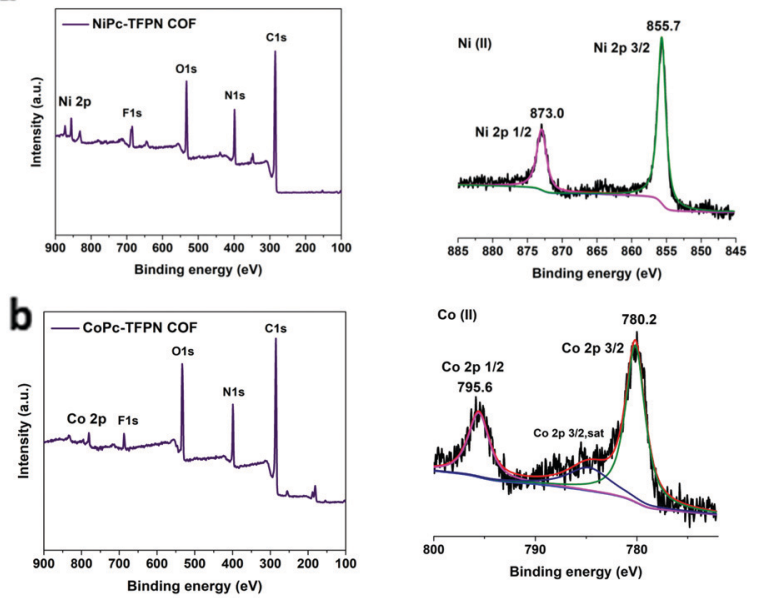

C

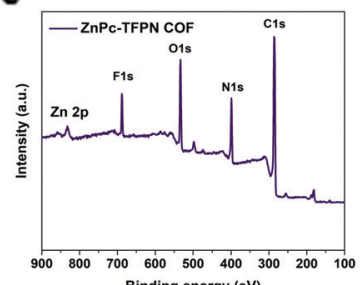

Binding energy (eV)
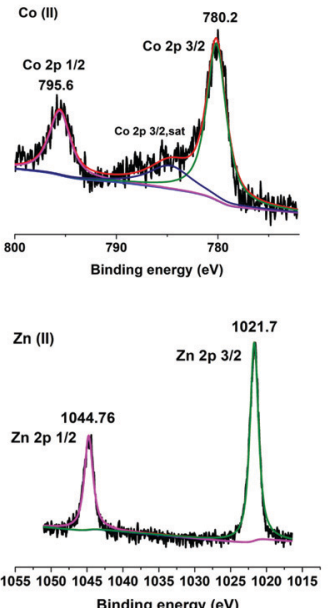

Binding energy (eV)
Fig. 46 Survey scan and high-resolution XPS profiles of (a) NiPc-TFPN COF, (b) COPc-TFPN COF and (c) ZnPc-TFPN COF. Reproduced with permission from ref. 171, Copyright John Wiley and Sons (2021).

attributed to the physisorption of nitrogen or argon on porous solids.

The type II and type III isotherms are particularly associated with non-porous or microporous materials, where the size of their pores is $>50 \mathrm{~nm}$. The type II sorption curve reveals the unrestricted mono- and multi-layered adsorption of inert gas over the surface, whereas the type III isotherm is restricted to only multi-layer adsorption. The type IV and V isotherms represent a hysteresis loop, where multilayer adsorption occurs on the mesoporous surface (pore size 2-50 $\mathrm{nm}$ ), suggesting the presence of mesopores in the material. The type VI isotherm suggests stepwise multilayer adsorption on an enormously homogeneous non-porous solid surface.

Various interesting examples of highly porous COFs with different linkages are discussed in this section, indicating their BET surface areas, number of pores, size and volume and the effect of functionalization.

It is very interesting to note that the highest BET surface area reported in COF chemistry is for the boronic ester-linked DBA-3D COF-1 with a value of $5083 \mathrm{~m}^{2} \mathrm{~g}^{-1}$. Its surface area was reduced down to $4763 \mathrm{~m}^{2} \mathrm{~g}^{-1}$ upon metallation of its framework. ${ }^{197}$

A distinctive example for the effect of alkyl chain length in boronic ester-linked COFs, namely COF-18 ̊ ( $\mathrm{R}=\mathrm{H})$, COF-16 ̊̊ $(\mathrm{R}=\mathrm{Me}), \mathrm{COF}-14 \AA(\mathrm{R}=\mathrm{Et})$ and COF- $11 \AA(\mathrm{R}=n-\mathrm{Pr})$, on the BET surface is shown in Fig. $48 \mathrm{a} \cdot{ }^{198}$ For an increase in the chain length from COF- $18 \AA$ to COF- $11 \AA$, the surface area is drastically reduced, having $\mathrm{SA}_{\mathrm{BET}}$ values of $1263,753,805$ and $105 \mathrm{~m}^{2} \mathrm{~g}^{-1}$, respectively, based on the type-I $\mathrm{N}_{2}$ gas adsorption isotherms at $77 \mathrm{~K}$. The pore diameter decreased from $18 \AA$ to $11 \AA$, as denoted by the name of the COF. It must be noted that the ethylsubstituted framework exhibits a slightly greater surface area in comparison to the methyl-substituted COF due to the rotation of the ethyl group, which provides a large surface area to adsorb nitrogen. A similar trend was observed for pore volume.

The triazine-linked CTF series, viz. CTF-1, CTF-1-600, FCTF1 and FCTF-1-600, was analyzed via Ar sorption isotherms at $87 \mathrm{~K}$ to study the effect of temperature conditions on their surface area (Fig. 48b). ${ }^{120}$ Both CTF-1 and FCTF-1 exhibited type-I sorption isotherms, with the microporous cumulative surface area of 461 and $623 \mathrm{~m}^{2} \mathrm{~g}^{-1}$, respectively. According to non-local density-functional theory (NLDFT), it was found that CTF-1 exhibits a pore size of $0.5 \mathrm{~nm}$, whereas FCTF- 1 has two pores, 0.46 and $0.54 \mathrm{~nm}$. On increasing the reaction temperature from 400 to $600{ }^{\circ} \mathrm{C}$ for the synthesis of CTF-1600 , a type IV sorption isotherm was observed, indicating that mesopores, was obtained with a surprisingly low surface area of $\sim 157 \mathrm{~m}^{2} \mathrm{~g}^{-1}$. However, FCTF-1-600 showed a type I isotherm with a surface area of $752 \mathrm{~m}^{2} \mathrm{~g}^{-1}$. The reduced pore size $\sim 0.59 \mathrm{~nm}$ for FCTF-1-600 is possibly due to the F-atom substitutions in its framework.

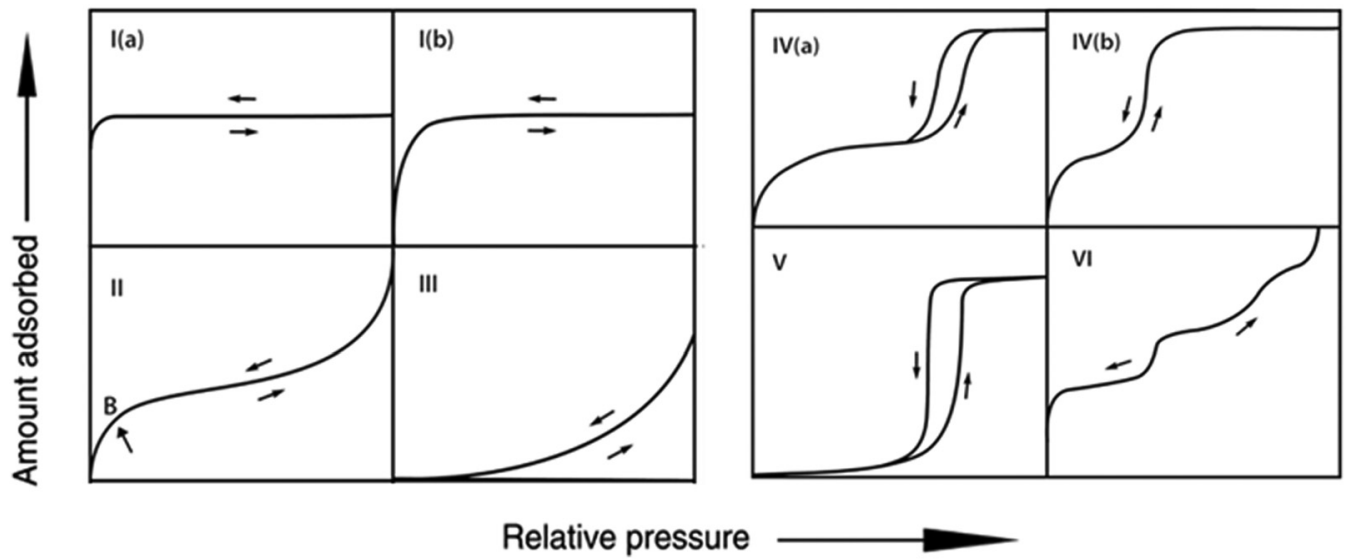

Fig. 47 Different types of BET adsorption isotherms. Reproduced and adapted from ref. 196 (no permission required), Copyright IUPAC (2015). 
a
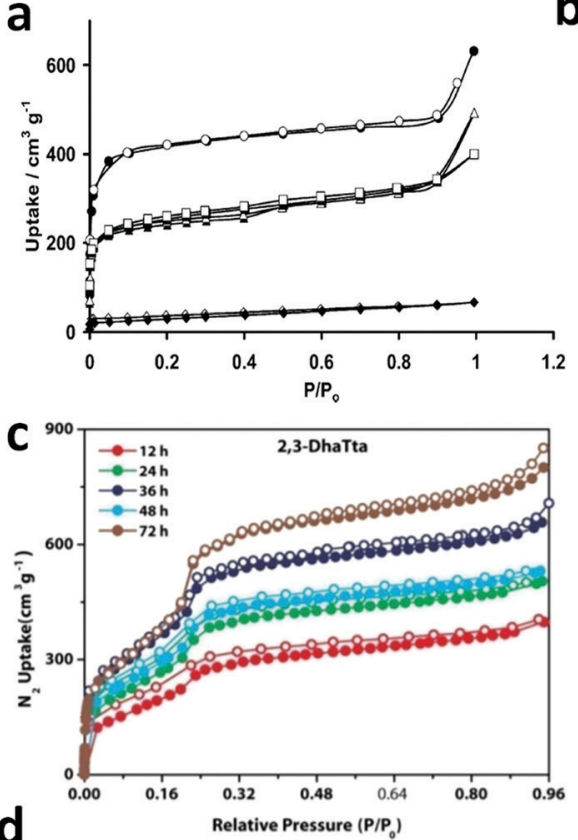

d

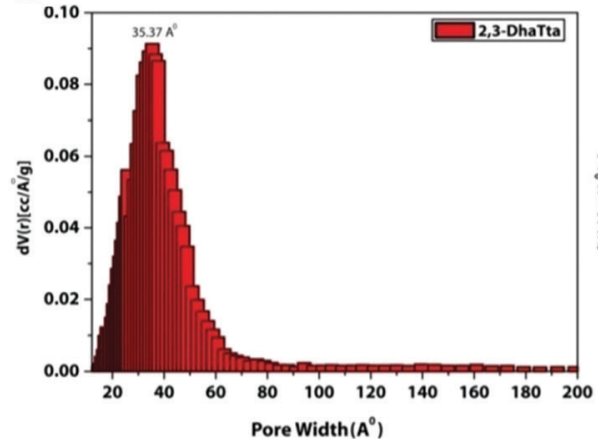

b
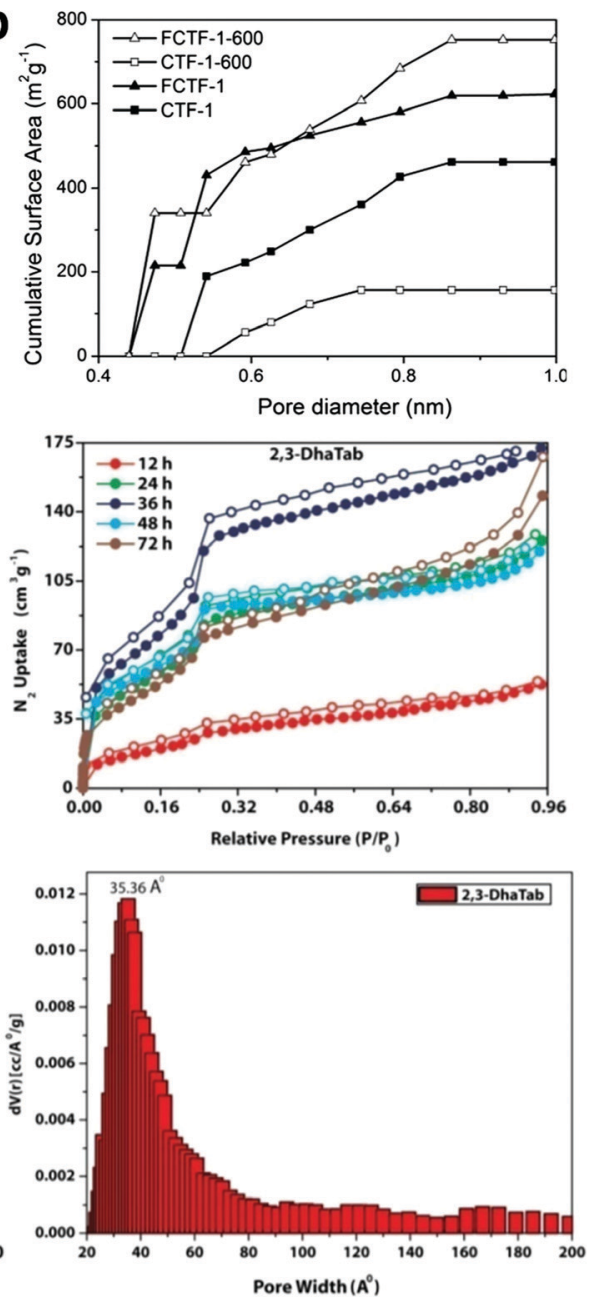

Fig. 48 Adsorption isotherm of (a) boronic ester-linked COFs, COF-18, , COF-16 $\AA$, COF-14 $\AA$ and COF-11 $\AA$, (b) triazine-linked COFs, CTF-1, CTF-1600, FCTF-1 and FCTF-1-600 and (c) imine-linked COFs, 2,3-DhaTta and 2,3-DhaTab with their pore size distribution (d). Reproduced with permission from: (a) ref. 198, Copyright John Wiley and Sons (2008); (b) ref. 120, Copyright The Royal Society of Chemistry (2013) and (c and d) ref. 79, Copyright John Wiley and Sons (2016).

This technique has also been utilized to monitor the significant changes within the framework caused due by different structural packing. On example was reported for the imine-linked 2,3-DhaTta and 2,3-DhaTab COFs (Fig. 48c and d), respectively. These two frameworks are different in terms of the $\pi-\pi$ packing among the 2D layers (see Section 3.2.1). Specifically, 2,3-DhaTta contains planar triazine-cored linker units, which create high crystallinity and high BET surface area in the framework. Its $\mathrm{N}_{2}$ adsorption isotherm depicts a type-IV curve, indicating a mesoporous framework with a surface area of $\sim 1700 \mathrm{~m}^{2} \mathrm{~g}^{-1}$. Conversely, 2,3-DhaTab possesses a low surface area of $\sim 413 \mathrm{~m}^{2} \mathrm{~g}^{-1}$, which is attributed to its reduced planarity and crystallinity because of its twisted phenyl-cores. This was further confirmed by the PXRD technique (vide Section 5.3.1). Moreover, a time-dependent study was conducted on both frameworks at $12,24,36,48$ and $72 \mathrm{~h}$, showing the framework building. ${ }^{79}$

The study of gas sorption and BET surface area is also helpful to support dimensionality changes and the extent of interpenetration. The pair 2D ILCOF-1 and 3D-ILCOF-1, which use planar $C_{4}$-symmetric and non-planar $T_{\mathrm{d}}$-symmetric aldehyde monomers, respectively, is a great example. According to the Ar sorption isotherm, ILCOF-1 has a type IV curve with a sharp uptake in the range of $P / P_{0}=0.05-0.15$, indicating the mesoporous nature of this material, whereas the $\mathrm{N}_{2}$ isotherm of the $3 \mathrm{D}$ framework depicts uptake below $P / P_{0}=0.1$, indicating its microporous cavities (Fig. 49a and b), respectively. Their BET surface areas are 2723 and $517 \mathrm{~m}^{2} \mathrm{~g}^{-1}$ with a pore volume of 1.21 and $0.36 \mathrm{~cm}^{3} \mathrm{~g}^{-1}$, respectively, which was calculated using the NLDFT method. The reason for the drastic reduction in the various surface parameters is the high degree of interpenetration in 3D-ILCOF-1. Moreover, the two isoreticular COFs, 3D-ILCOF-2 and 3D-ILCOF-3, show a slightly higher adsorption uptake in comparison to 3D-ILCOF-1. ${ }^{85,122}$

The hydrazone- and urea-based COFs, Pythz and Pyurea COF, display quite different $\mathrm{N}_{2}$ sorption behaviours at $77 \mathrm{~K}$ (Fig. 49c). ${ }^{138}$ In the case of the Pythz COF, a distinctive combination of type I and II isotherms was observed, indicating its both micro and mesoporous nature. Conversely, the Pyurea COF shows a type I 

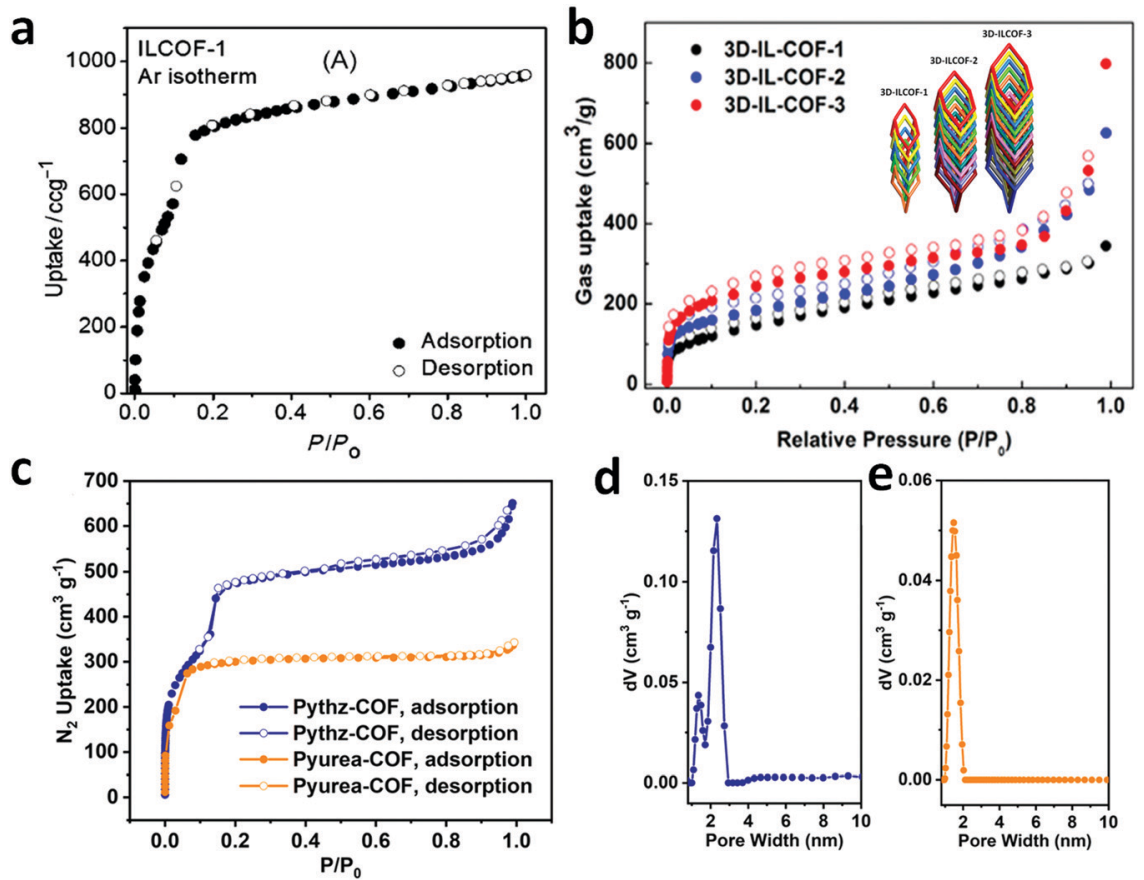

Fig. 49 Adsorption isotherms of imine-linked COFs (a) ILCOF-1 and (b) 3D-ILCOF-1/2/3, and (c) hydrazone-linked Pythz-COF and Pyurea-COF with their pore-size distribution curves (d and e). Reproduced with permission from: (a) ref. 122, Copyright John Wiley and Sons (2013); (b) ref. 85, Copyright the American Chemical Society (2018) and (c-e) ref. 138, Copyright The Royal Society of Chemistry (2021).

adsorption isotherm, indicating its purely microporous structure. The BET surface area/pore volume of these frameworks were 1571/ 1.21 and 1016/0.53 $\mathrm{m}^{2} \mathrm{~g}^{-1} / \mathrm{cm}^{3} \mathrm{~g}^{-1}$, respectively. By using NLDFT calculations, the pore size distribution confirmed two pore sizes $(2.34$ and $1.36 \mathrm{~nm})$ for Pythz COF and one pore size $(1.48 \mathrm{~nm})$ for the Pyurea COF (Fig. 49d and e), respectively.

Two COFs, amial-COF-1 and aminal-COF-2, show similar a surface area of $\sim 1168 \mathrm{~m}^{2} \mathrm{~g}^{-1}$ but different pore volumes of 0.65 and $0.89 \mathrm{~cm}^{3} \mathrm{~g}^{-1}$, respectively, at $P / P_{0}=0.99$. By using quenched solid-state density functional theory (QSDFT), the calculated pore size distributions (Fig. 50) show that both COFs have two types of micropores, i.e., at 5.2 and 8.2 for aminalCOF-1 and 5.4 and 8.2 for aminal-COF-2 (Fig. 50). ${ }^{38}$

It has been seen that by reticulation of the framework, the specific surface areas and pore dimensions change. This can be numerically compared by the BET surface area and pore sizes of the different COFs shown in Table 3.

\subsection{Thermogravimetric analysis (TGA)}

One of the major reasons for the development of COFs is to generate rigid and robust materials that can withstand high thermal conditions. TGA is used to detect the maximum temperature up to which the polymer is stable, where the percentage weight loss of the framework over a certain temperature range $\left(\sim 30-600{ }^{\circ} \mathrm{C}\right)$ is calculated. This also provides an indication for the presence of solvent molecules used during the synthesis and/or any residual unreacted monomers within the framework lattice. Accordingly, the as-synthesized COF sample is fully activated at high temperature $\left(\sim 100-120^{\circ} \mathrm{C}\right)$ under vacuum, and then subjected to TGA analysis under $\mathrm{N}_{2}$ environment.
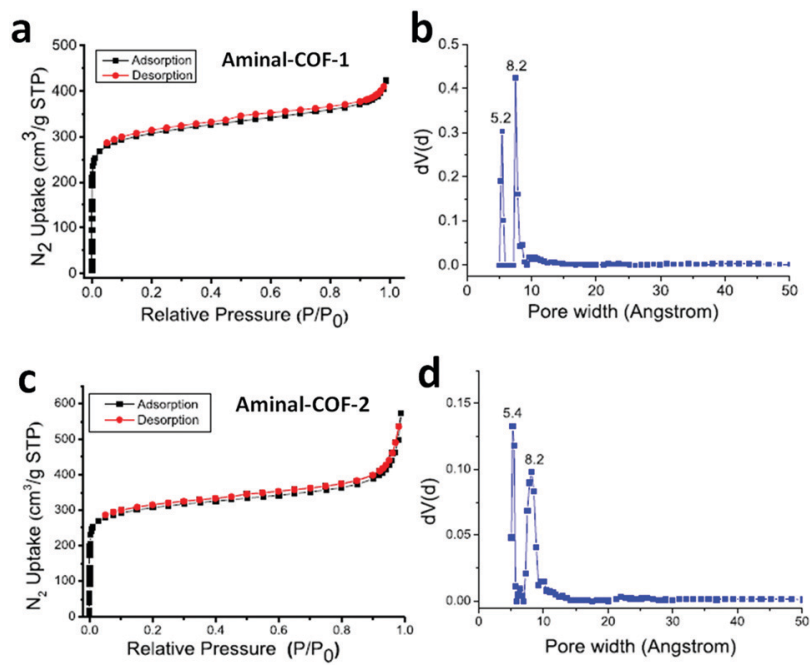

Fig. 50 Adsorption isotherm of (a) aminal-COF-1 and (c) aminal-COF-2 with their pore-size distribution using QSDFT ( $b$ and d), respectively. Reproduced with permission from ref. 38, Copyright the American Chemical Society (2019).

The triazine-connected crystalline framework show very high thermal stability in the presence of air. For example, CTF-0 is relatively more stable than CTF- 1 up to $600{ }^{\circ} \mathrm{C}$, which indicates the rich nitrogen content and good cross linking density in CTF-0 compared to CTF- $1 .{ }^{19}$ It should be noted that only triazine-based COFs exhibit impressive thermal stability in realistic conditions, i.e., in the presence of an oxygen atmosphere. For all other linkages, the TGA is recorded under an inert atmosphere of $\mathrm{N}_{2}$ gas due to their low stability. 
Both activated $\beta$-ketoenamine-linked TpPa- 1 and TpPa- 2 are thermally stable up to $350{ }^{\circ} \mathrm{C}$, indicating the absence of guest molecules within their pores. However, with a further increase in temperature, there is a large weight loss (TpPa-1: 40\% and TpPa-2: $50 \%$, which is attributed to their structural decomposition. ${ }^{67}$

The TGA analysis of hydrazone-linked COFs, such as NUS-50 and NUS-51, showed their thermal stability up to $350{ }^{\circ} \mathrm{C} .{ }^{130}$ The aminal-linked COFs, namely aminal COF-1 and aminal COF-2, are comparatively less thermally stable. Their TGA data revealed virtually no weight loss up to $200{ }^{\circ} \mathrm{C}$, and only $10 \%$ weight loss was observed at around $320{ }^{\circ} \mathrm{C} .{ }^{38}$

The stability changes during post-synthetic modification of the robust organic framework can also be monitored using this technique. The $\mathrm{COF} \operatorname{TpBD}\left(\mathrm{NO}_{2}\right)_{2}$ upon reduction to form $\operatorname{TpBD}\left(\mathrm{NH}_{2}\right)_{2}$ showed a similar weight loss profile (weight loss was observed at $450{ }^{\circ} \mathrm{C}$, suggesting the decomposition of the framework), indicating that there were no distinct structural changes. ${ }^{146}$ The TGA analysis of COF-701 shows no significant weight loss before $400{ }^{\circ} \mathrm{C}$ under a nitrogen atmosphere, proving its exceptional stability. ${ }^{160} \mathrm{~A}$ rare example of the highly crystalline imine-linked 2D ILCOF-1, which remains stable up to $400{ }^{\circ} \mathrm{C}$ even under aerobic conditions, was also reported. ${ }^{122}$

The above-mentioned characterization techniques are greatly helpful to discover the attractive properties of the N-rich frameworks and guide their use for suitable and potential applications.

\section{Luminescence and sensing}

\subsection{Luminescence}

In this section, we introduce and discuss a few phenomena that are essential to describe the luminescence properties of COFs and explain their mechanism of action in various applications in Section 6.2.

6.1.1 Increased $\pi$-conjugation. According to the basic spectroscopic knowledge, it is known that by increasing the length of a $\pi$-conjugated system, the energy gap between the valence and conduction band is lowered, leading to more redshifted $\pi-\pi^{*}$ transitions ( $\Delta \lambda$ higher, $\Delta E$ lower). This further affects the fluorescence emission efficiency ( $\Phi_{\mathrm{f}}$ ) of the conjugated system, which is directly proportional to the length of the conjugated system. ${ }^{199}$ Nevertheless, COFs have been explored as highly fluorescent materials by joining strongly emissive monomers and extending their conjugation via different linkages (vide supra). This effect was studied by Zhao et al., where a highly conjugated TP-COF was constructed via the $\left[C_{3}+C_{2}\right]$ combination of 2,4,6-tris(4-formylphenyl)-1,3,5-triazine (TFPT) and 2,2'-(1,4-phenylene)diacetonitrile (PDAN). ${ }^{200}$ It was observed that there was a reduction in energy gap going from the model compound (TFPT + phenylene acetonitrile) to TP-COF, which led to both a red-shifted absorbance and emission maximum from 355 and $506 \mathrm{~nm}$ to 410 and $543 \mathrm{~nm}$, respectively. This is solely ascribed to the extended $\pi$-conjugation during the formation of the framework.

Furthermore, when the number of consecutive phenyl rings increases within a unit, it loses planarity, giving rise to an anomalous trend. For instance, Perepichka and co-workers reported two different COFs, namely 3PD and 3BD, synthesized from a combination of a tritopic- $b$-ketoenol and two different aromatic amine units, i.e., phenylenediamine (PD) and biphenyldiamine (BD). ${ }^{201}$ The calculated band gap for the less $p$-conjugated COF system, 3PD, is $2.03 \mathrm{eV}$, which, on the contrary, increased to $2.19 \mathrm{eV}$ when the conjugation within the framework increased, i.e., in 3BD COF. This is attributed to the loss of planarity between the phenyl rings of the linker BD.

A similar example was reported by Jiang et al., examining the details of $\pi$-conjugation and its effect on the luminescent properties of COF materials. ${ }^{202}$ A pyrene-containing $C_{4}$-symmetric monomer unit, tetraformylphenylpyrene (TFFPy), was used to synthesize three $\mathrm{sp}^{2}$-carbon conjugated 2D-COFs, viz., $\mathrm{sp}^{2} \mathrm{c}-\mathrm{COF}, \mathrm{sp}^{2} \mathrm{c}-\mathrm{COF}-2$ and $\mathrm{sp}^{2} \mathrm{c}-\mathrm{COF}-3$, via their combination with $C_{2}$-symmetric phenyl, biphenyl and triphenylacetonitrile units (PDAN, BPDAN and TPDAN), respectively. In accordance with the theory, there was a bathochromic shift ( $\Delta \lambda$ higher) in the emission spectra of TFFPy and three COFs as the monomer emits blue light, whereas the COFs emit in the bright red region (orange, bright yellow, dull and yellow). Conversely, the absorbance and emission spectra of $\mathrm{sp}^{2} \mathrm{c}-\mathrm{COF}-2$ and $\mathrm{sp}^{2} \mathrm{c}-\mathrm{COF}-3$ were more blue-shifted despite their higher conjugated frameworks. This is due to the deplanarization of the monomeric unit when more phenyl rings are incorporated within one row. Therefore, a hypsochromic shift was seen, corresponding to a high transition energy band gap.

6.1.2 Aggregation-induced emission (AIE). The study of luminescence not only includes insight about radiative emission, where knowledge of the non-radiative emission pathways can also be gained. These pathways can be examined under different conditions such as molecular aggregation, their steric environment and solvent surroundings. Several moieties have been reported to be luminescent active but do not show any FL emission in a particular environment, as shown in Fig. 51. One of the radiationless pathways is aggregation-caused quenching (ACQ), in which the quenched luminescence is attributed to the tendency of molecules to aggregate. A typical example of the ACQ effect is the perylene molecule, which shows strong luminescence when diluted with THF solvent. ${ }^{203}$ However, upon the addition of a solvent in which it is much less soluble, particularly water, their molecules form excimers via $\pi-\pi$ interactions, leading to a wellpacked structure. Consequently, the luminescence is weakened or completely quenched. On the contrary, the tetraphenylethene (TPE) molecule inhibits the luminescence properties in the dilute

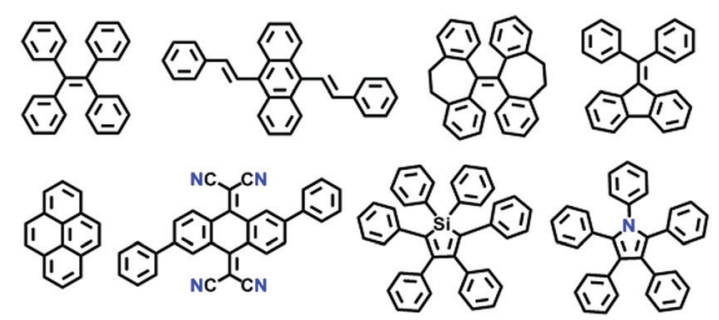

Fig. 51 Selected examples of commonly used fluorophore units that show the AIE effect. 
state but emits when its molecules aggregate. ${ }^{204,205}$ In the dilute state, the four phenyl rings of TPE undergo dynamic rotations against the static olefinic double bond, causing thermal dissipation of energy via a non-radiative pathway. When the concentration of TPE molecules is high, they aggregate and restrict intramolecular rotations (RIR), showing a strong emissive property. This process is called aggregation-induced emission (AIE). These molecules are called AIE luminogens, or simply AIEgens (terminologically, "luminophores" are the emissive chromophores, whereas "luminogens" are the non-emissive chromophores that emit under particular conditions, such as upon aggregation).

The AIE effect has been observed in covalent organic frameworks incorporating AIEgens as monomeric units. Since the COF layers are stacked on each other via non-covalent interlayer $\pi$-interactions, the fluorescent moieties present within the framework form excimers, leading to the dissipation of energy via a non-radiative pathway. This eventually restricts the luminescence in COFs due to the ACQ effect. To turn off this process, aggregation-induced emission (AIE) becomes functional, where a radiative emission competes with the faster non-radiative process to impede intramolecular rotations (RIR), vibrations (RIV) and molecular motions (RIM).

On coating the 3D-TPE-COF, which was reported by Ding et al., on a regular blue LED, bright white light was emitted, which makes the COF a white light-emitting diode (WLED). ${ }^{206}$ The photoluminescence properties of the TPE monomer were studied when it was incorporated inside the framework. It was observed that the model compound of TPE-COF exhibited very weak yellow-green emission with a low photoluminescent quantum yield (ca. 6.6\%), whereas the COF itself emitted bright yellow light $\left(\lambda_{\max }=543 \mathrm{~nm}\right)$ with a much higher quantum yield (ca. 20\%). This is due to the restricted intramolecular rotation (RIR) of the phenyl ring rotators alongside the olefinic bond in TPE when placed in the rigid skeletal three-dimensional framework.

Likewise, the 2D TPE-Ph-COF contains a similar TPE-based core, which shows strong luminescence emission in its aggregated state. A boronic ester-linked COF was reported by Jiang and co-workers in 2016, elaborating the emission of bright blue color at $500 \mathrm{~nm}$ when excited at $390 \mathrm{~nm}$ (Fig. 52). The small COF crystallites from a well-defined belt-like shape with an average size of $4 \mu \mathrm{m}$, the formation of which was monitored by timedependent morphology growth. ${ }^{29}$

Other examples of COFs containing TPE AIEgens are SIOCCOF- $x(x=1,2,5$, and 6$),{ }^{207,208} 4 \mathrm{PE}-x \mathrm{P}-\mathrm{COF}(x=1,2$, and 3$)$ and 4 PE-TT ${ }^{27}$ NUS $30-32^{209}$ and COF-DHTA, DETA and DBTA, where the terephthaldehyde unit is substituted with hydroxyl, ethoxy and butoxy groups, respectively. ${ }^{210}$

The pyrene moiety has also attracted extensive attention as an AIEgen when utilized as a monomer in COFs. For instance, Jiang and co-workers reported HHTP-PDBA-COF constructed via the $\left[C_{3}+C_{2}\right]$ polycondensation of hexahydroxytriphenylene (HHTP) and pyrenediboronic acid (PDBA). ${ }^{211}$ A strong emission at $474 \mathrm{~nm}$ from the pyrene moieties was observed when packed in an eclipsed fashion. One year later, the same group synthesized 2D polypyrene sheets named PPy-COF via the self-condensation of PDBA under solvothermal conditions. ${ }^{17}$ Due to the layer alignment of the pyrene units in the framework, excimer formation occurred, leading to a strong luminescence emission at $484 \mathrm{~nm}$.

Later, Crowe et al. reported Py-DBA-COF-1 and Py-DBA-COF-2, which were synthesized via the condensation reaction of two different dehydrobenzoannulene units, DBA[12] and DBA[18], with pyrenediboronic acid, respectively. ${ }^{212}$ The DBA[12] and [18] units are planar, $\pi$-conjugated emissive macrocycles, where 12 and 18 indicate the number of carbon atoms within their triangular pores. A mixed variant COF using DBA[12] and [18] and PDBA was also synthesized, namely Py-MV-DBA-COF. It was found that the AIE effect of the DBA units resulted in a strong emission property for all the COFs. However, a huge red-shifted emission was observed for COF-1 (530 nm) and the mixed variant COF (528 nm) with respect to $\mathrm{COF}-2(483 \mathrm{~nm})$, which is ascribed to the fact that the DBA[12] unit embedded in the framework shows symmetry-forbidden transitions in its ground-state. This leads to the dominant excited-state properties of Py-DBA-COF-1 and Py-MV-DBA-COF.

A few more pyrene-containing COFs possessing the AIE effect have been reported in the literature, viz., A-TEBPY-COF, A-TENPY-COF, A-TEPPY-COF ${ }^{213}$ 3D-Py-COF,${ }^{46}$ Car-ETTA-COF, TFFPy-ETTA COF ${ }^{214}$ COF-JLU- $2 x(x=3,4,5),{ }^{215}$ Per-Py-COF. $^{216}$
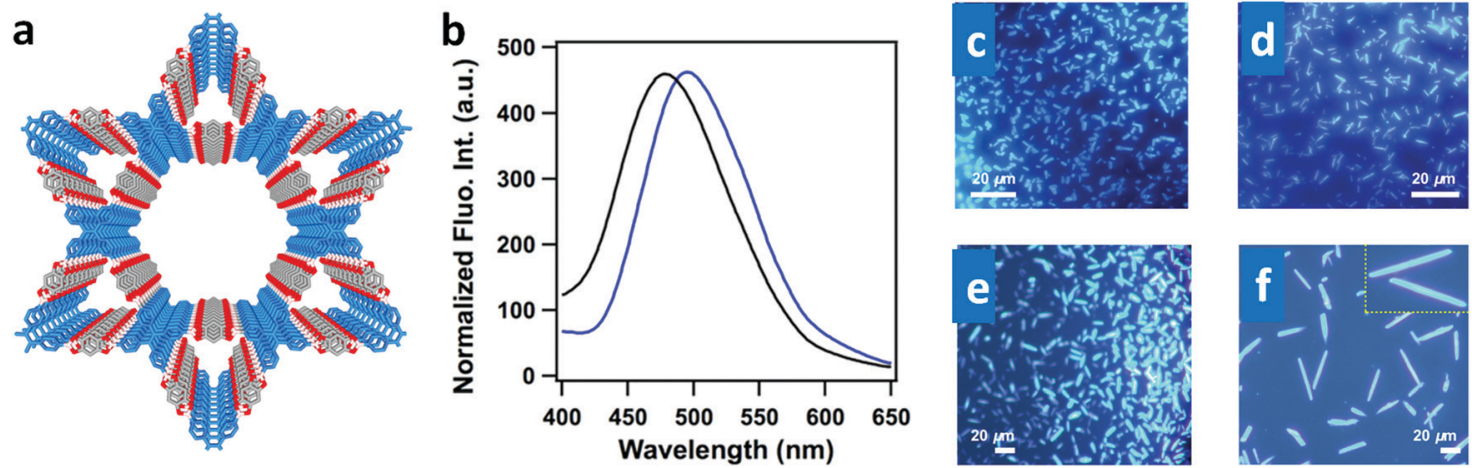

Fig. 52 (a) Structure of TPE-Ph-COF with kgm topology. (b) Fluorescence spectra of the TPE model compound (black) and the TPE-Ph COF (blue). Fluorescence microscopy images of TPE-Ph COF samples prepared at different reaction times of (c) 3 days, (d) 10 days, (e) 20 days, and (f) 30 days (inset image showing an enlarged view of the belts). Reproduced with permission from ref. 29, Copyright the American Chemical Society (2016). 

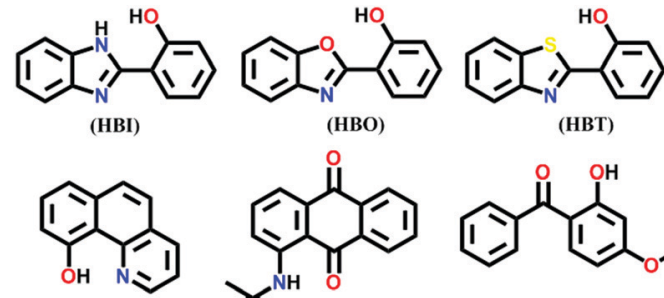

(Quinoline)
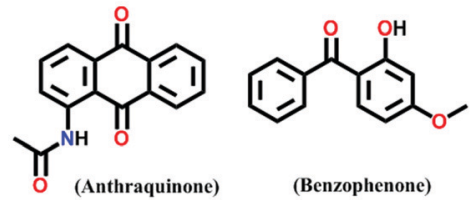

Fig. 53 Selected examples of commonly used ESIPT fluorophore units.

6.1.3 Excited-state intramolecular proton transfer process (ESIPT). This has been extensively studied in molecules containing proton donor and acceptor moieties in close proximity to each other. This mechanism was first discovered in salicylic acid and its derivative methyl salicylate by Weller in $1955 .^{217}$ Since then, numerous studies have been performed and the importance of ESIPT in showing luminescence has been exploited. ${ }^{218}$ Certain fluorophores have been reported in the literature to exhibit the ESIPT mechanism, such as 2-hydroxyphenyl-substituted benzimidazol (HBI), benzoxazole (HBO) and benzothiazole (HBT) derivatives, quinoline and anthraquinone derivatives, and benzophenone derivatives, as shown in Fig. 53.

Recently, various COFs have been reported in which free $-\mathrm{OH}$ and $-\mathrm{NH}_{2}$ groups are present near the proton acceptor sites, such as imine or triazine linkages or $\mathrm{C}=\mathrm{O}$ groups, which leads to intramolecular hydrogen-bond interactions and turns on the ESIPT process. The monomeric units exploited to make highly luminescent COFs following the ESIPT mechanism are shown in Fig. 54.

This photochemical switching process involves four states, i.e., electronic ground state and first excited state of enol and keto forms, denoted by $\mathrm{E}\left(\mathrm{S}_{0}\right)$ and $\mathrm{K}\left(\mathrm{S}_{0}\right)$ and $\mathrm{E}^{*}\left(\mathrm{~S}_{1}\right)$ and $\mathrm{K}^{*}\left(\mathrm{~S}_{1}\right)$, respectively. The enol form of the ESIPT fluorophore is seen at the $\mathrm{E}\left(\mathrm{S}_{0}\right)$ level, which after photon absorption gets excited to the $\mathrm{E}^{*}\left(\mathrm{~S}_{1}\right)$ level. At this stage, there is an increase in the acidic character in the $\mathrm{H}$-bond donor moiety and basicity in the H-bond acceptor site, which causes an extremely rapid ESIPT transition from the excited enol to keto form at the rate of $\sim 10^{12} \mathrm{~s}^{-1}$. After radiative decay from this stage $\left(\mathrm{K}^{*}\left(\mathrm{~S}_{1}\right)\right.$ to $\mathrm{K}$ $\left(\mathrm{S}_{0}\right)$ ), a reverse proton transfer (RPT) possibly happens, giving<smiles>O=Cc1cc(O)c(C=O)cc1O</smiles><smiles>O=Cc1c(O)c(C=O)c(O)c(C=O)c1O</smiles><smiles>O=Cc1cc(C=O)c(O)c(C=O)c1O</smiles><smiles>NNC(=O)c1cc(C(=O)NN)cc(C(=O)NN)c1</smiles><smiles>NNC(=O)c1ccc(C(=O)NN)cc1</smiles><smiles>CCOc1cc(C(=O)NN)ccc1OCCOc1cc(C(=O)NN)ccc1C(=O)NN</smiles>

Fig. $54 C_{2}$ - and $C_{3}$-Symmetric monomer units for establishing ESIPT the mechanism in COFs.

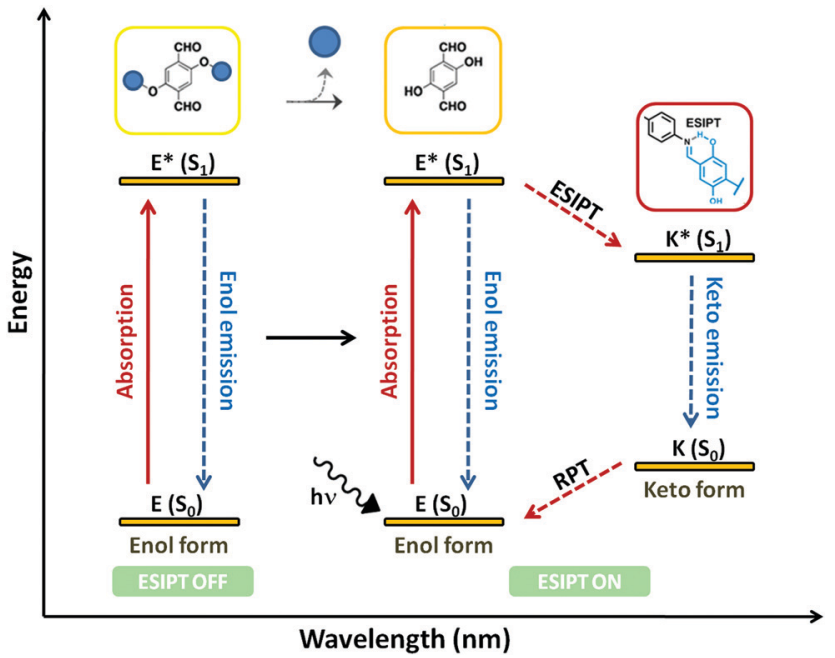

Fig. 55 General mechanism showing the dual-enol and keto emission in the excited-state intramolecular proton transfer (ESIPT) process in COFs.

the original enol form. A detailed scheme of this process is shown in Fig. 55.

The ESIPT mechanism has been widely studied with a large variety of organic and inorganic molecules and polymers. ${ }^{218-222}$ During this study, it has been found that the surrounding environment of these materials highly affects their luminescence property. For instance, polar solvents form additional hydrogen bonds with the molecules in their enolic form, competing with the intramolecular hydrogen-bond formation. This ceases the ESIPT pathway from the enol to keto form, hence inhibiting the process. Thus, organic solvents have been largely used as a medium to produce and examine the luminescence caused by the excited-state intramolecular proton transfer mechanism. ${ }^{218}$

An example of a COF (IISERP-COF-7) showing dual-emission through the ESIPT mechanism was reported by Halder et al. (Fig. 56). ${ }^{223}$ It was found to be a white-light emitter in various solvents. The free $-\mathrm{OH}$ groups on the $C_{3}$-unit form intramolecular hydrogen-bonding with the $-\mathrm{CH}=\mathrm{N}$ - linkages and strong enol-keto tautomerism was observed. The appropriate geometry of the $-\mathrm{OH}$ group and its orientation towards the imine linkage turns on the ESIPT mechanism.

However, this dual-emission is highly disturbed by polar $\mathrm{N}$-donor (pyridine, picoline, and acetonitrile) and O-donor (THF, dioxane, methanol and ethanol) solvents. It has been observed that highly basic N-donor solvents help in proton transfer from the enolic to keto form, whereas the enol form predominates when O-donor solvents are employed as the medium. In the latter case, the phenolic hydrogens of the resorcinol moiety are sandwiched between the O-atom of the solvent and phenolic oxygen.

The combination of an ESIPT and ICT system responsible for dual-emissive covalent organic frameworks was reported by Yan and co-workers. ${ }^{222}$ The TzDa-COF contains a triazine docking site and an intramolecular hydrogen-bonding site. Upon excitation at 365 and $425 \mathrm{~nm}$ in an ethylacetate solution, the TzDa COF exhibits fluorescence emissions at 500 and $590 \mathrm{~nm}$. The phenomenon of ICT was observed in the Tz moiety, where charge transfer 

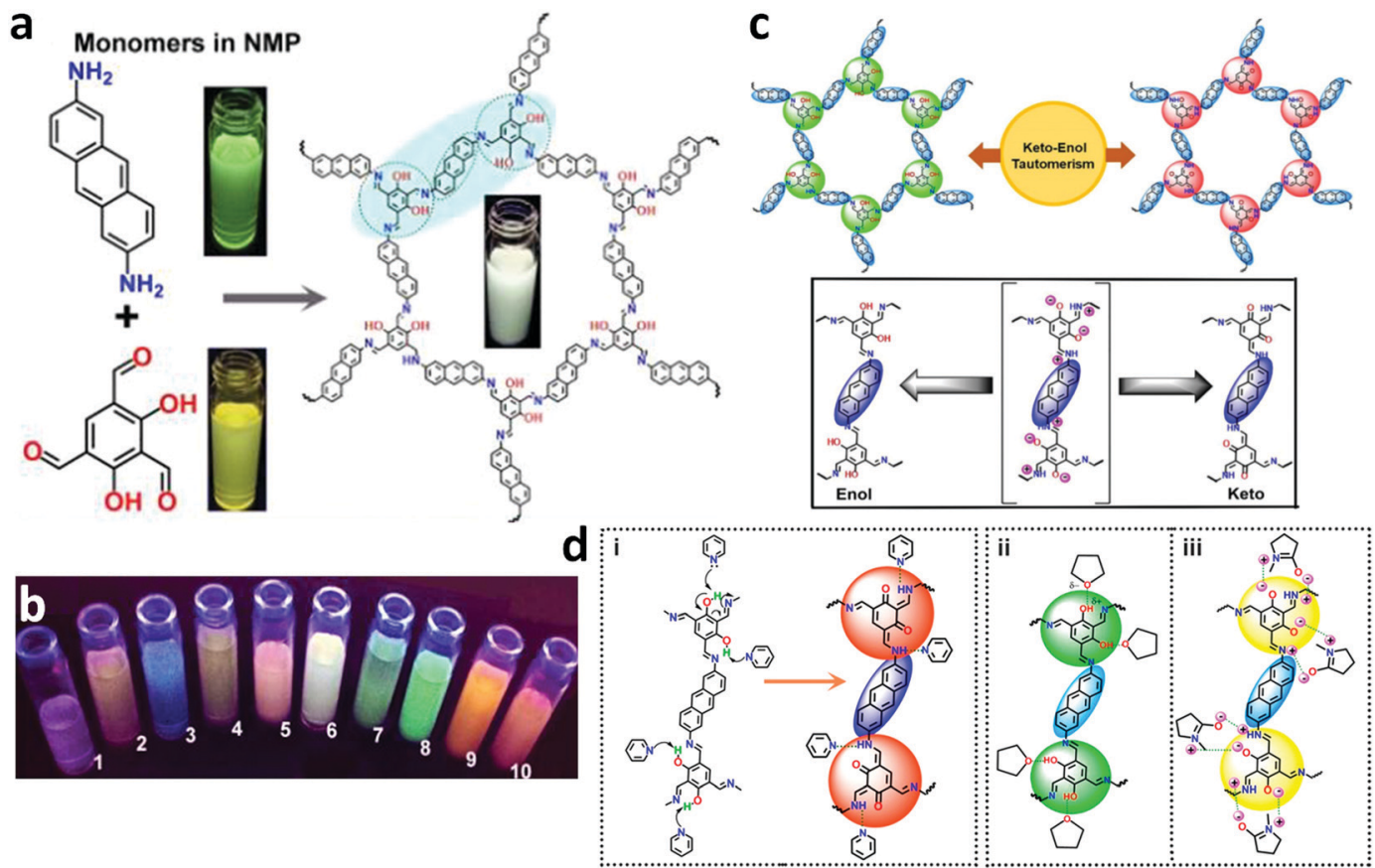

Fig. 56 (a) Dispersions of the monomers and COF suspended in NMP pictured under a UV lamp. (b) COF dispersion in different solvents placed under a UV lamp. Codes: 1, THF; 2, dioxane; 3, glyoxal; 4, pyridine; 5, DMA; 6, NMP; 7, ethanol; 8, formamide; 9, picoline; and 10, DMF. (c) Proposed schematic representation of keto-enol tautomerism in the resorcinol unit of the COF (top). The repeating unit of the COF transforms from the keto to enol form and vice versa through a polarizable transition state (bottom). (d) (i) Proton abstraction mechanism in pyridine, which stabilizes the keto-form; (ii) hydrogen bonding by THF stabilizes the enol-form; and (iii) polarized NMP stabilizing the intermediate keto-enol form. Reproduced with permission from ref. 224 , Copyright the American Chemical Society (2018).

from the donor phenyl group and electron-deficient triazine center occurs, showing an emission at $500 \mathrm{~nm}$. The emission at $590 \mathrm{~nm}$ corresponds to the ESIPT effect due to the presence of a proton donor $-\mathrm{OH}$ group and a proton acceptor $-\mathrm{CH}=\mathrm{N}$ - bond in the $\mathrm{Da}$ moiety. Moreover, the effect of aqueous medium on the dualluminescence emission made the TzDa-COF capable of sensing trace water molecules in organic solvents. It was observed that an increase in water content from 0.6 to $3.2 \mathrm{wt} \%$ blocked the ICT process by forming additional hydrogen bonding between the water and triazine nitrogens. Conversely, the increased interactions of the water molecules with the Da moiety change its excitedstate form and increase the corresponding luminescence intensity at $590 \mathrm{~nm}$.

A unique example was reported by Loh and co-workers, where they exploited the inter and intralayer hydrogen bonding in 2D stacked COF sheets to induce emissive properties in the framework. ${ }^{224}$ For this, a hydrazone-based non-emissive building unit with various functionalities substituted on it was employed, named 2,5-disubstituted terephthalohydrazide (DHzDR, R = Me, All, and Pr). It was proposed that due to the free intramolecular bond rotations, the monomeric units do not emit. The moiety binds with different trialdehydes, such as benzene-1,3,5-tricarboxaldehyde ( $\mathrm{Tf}$ ) and tri(4-formylphenyl)benzene (TFPB), leading to the formation of $2 \mathrm{D}$ COFs with face-to-face stacked sheets, which tend to form interlayer hydrogen bonds between the free - $\mathrm{NH}$ groups of the hydrazide moiety in one layer with $\mathrm{C}-\mathrm{O}-\mathrm{C}$ sites in the other layer. Thus, the monomers cease their rotational motion, leading to strong quenching via the AIE-effect. Moreover, the ESIPT mechanism was also seen in the framework where dual-emission is caused by intralayer hydrogen bond formation between the $-\mathrm{NH}$ and imine linkage located at the proximal ends. Thus, both singleand dual-emission can be seen within a molecule via two different mechanisms.

In this context, COFs such as COF-4-OH ${ }^{225}$ and PEG-COF$42^{226}$ show significant dual-emission by rapid intramolecular proton transfer from the enolic excited state to the ketonic excited state and have been explored for the ESIPT mechanism. Close proximity of the proton donor and acceptor sites, solvent environment, etc., are essential factors that make the ESIPT process possible.

This aforementioned knowledge has pushed the sensingbased study of covalent organic frameworks forward and these materials have been widely exploited to detect various analytes, such as nitroaromatic explosives, various toxic anions and metal ions, volatile small molecules such as organic or inorganic solvents, and biosensing. In this field, it is exciting when COFs are utilized to sense different $\mathrm{pH}$ in aqueous media and for thermal sensing.

6.1.4 Photo-induced electron and energy transfer (PET). This is a process in which an electronically excited molecule passes its energy to another molecule. The excited molecule shows significant luminescence property and is called a fluorophore, and the other molecule is a quencher. Fig. 57 represents a schematic of the PET process in electron-rich/poor donor species. In the initial step, the ground-state fluorophore molecule (F) 


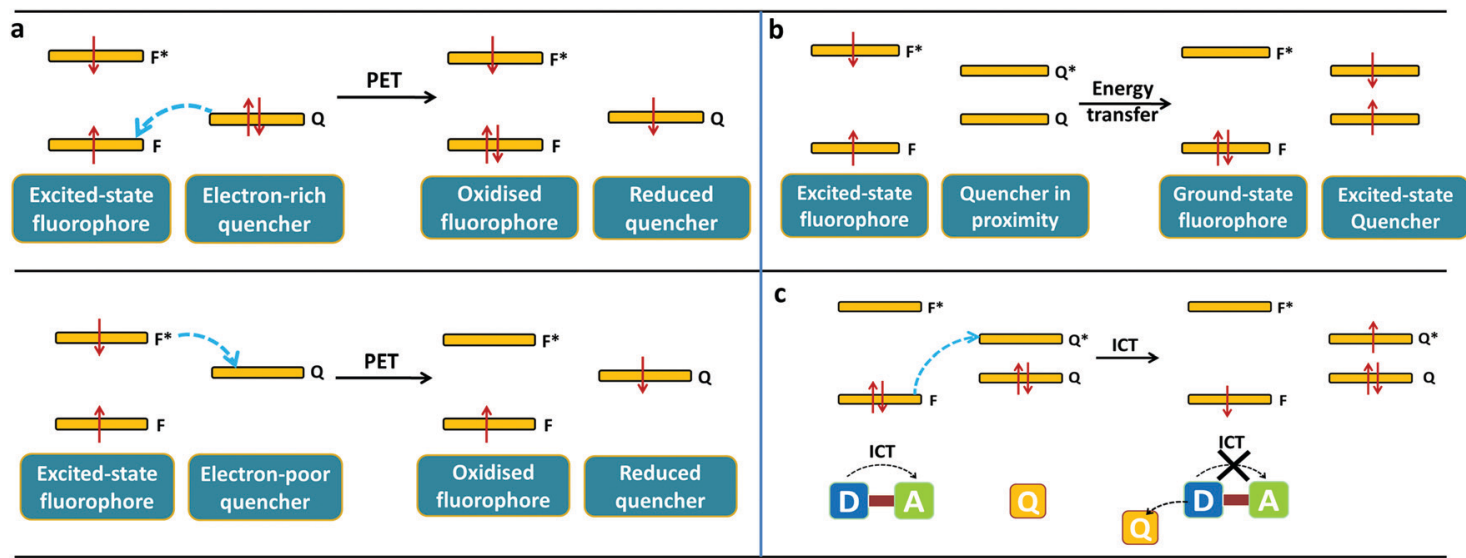

Fig. 57 Electron transfer (a) and energy transfer (b) quenching of a chromophore in the excited state. (c) Intramolecular charge transfer (ICT) mechanism.

absorbs energy from photons and upon irradiation gets excited to a higher electronic state, $\mathrm{F}^{*}$. When the excited-state fluorophore $\left(\mathrm{F}^{*}\right)$ comes in close proximity with electron-rich or electron-poor quenchers, electron transfer occurs, which leads to FL quenching in the system (shown in Fig. 57a and b). For this, the frontier orbital energies of the fluorophore and the quencher should be similar. On the contrary, the energy transfer mechanism for the FL quenching of a fluorophore does not involve any electron transfer from the excited electronic state $\mathrm{F}^{*}$. Rather, the excited state energy is transferred to the quencher whose LUMO must have lower energy values with respect to the excited state $\mathrm{F}^{*}$. Fig. $57 \mathrm{c}$ depicts the energy transfer process.

6.1.5 Intramolecular charge transfer (ICT). This charge transfer process involves the transfer of electronic charge from electron-donating species to the electron-accepting species (Fig. 57c). When both moieties lie inside the sample molecule, charge transfer occurs through a $\pi$-electron bridge, which is called intramolecular charge transfer (ICT). The charge transfer is affected by the solvent polarity and quenched in the presence of analytes, which act as an FL quencher. A polar solvent tends to stabilize the charge separation in an excited fluorophore. Conversely, the quencher can act as a donor or an acceptor depending on its electron-rich and electron-deficient nature.

6.1.6 Förster resonance electron transfer (FRET). Another very important process in fluorescence spectroscopy is Förster (or fluorescence) resonance energy transfer (FRET). According to FRET, when a fluorophore (called donor D) molecule and a quencher (called acceptor A) molecule couple through a dipoledipole interaction at a Förster distance in the range of 15-60 $\AA$, there is an overlap between the emission spectrum of the fluorophore and the absorption spectrum of the quencher. This process does not involve the emission of light by the donor, which is further reabsorbed. ${ }^{227}$ The spectral overlap is shown in Fig. 58. The shorter the distance between the two species, the greater the interaction between them. The extent of energy transfer, $\kappa_{\mathrm{T}}(r)$, is given by the following equation:

$$
\kappa_{\mathrm{T}}(r)=\frac{1}{\tau_{\mathrm{D}}}\left(\frac{R_{0}}{r}\right)^{6}
$$

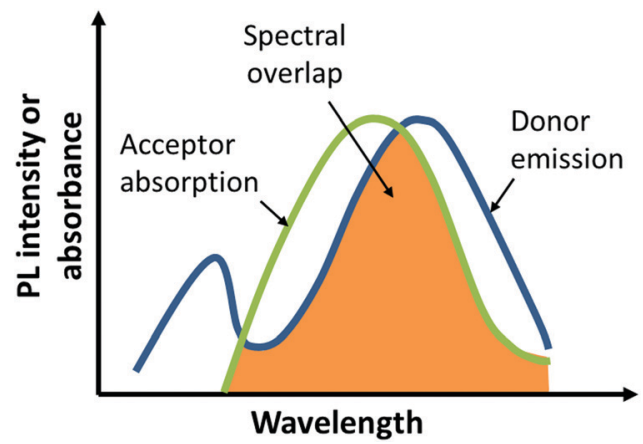

Fig. 58 Spectral overlap of donor emission and acceptor absorption.

where $r$ is the distance between the fluorescent donor (D) and quencher/acceptor (A), $R_{0}$ is the Förster distance and $\tau_{\mathrm{D}}$ is the lifetime of the donor in the absence of energy transfer.

This mechanism is widely seen in fluorescent-active COFs, and the potential candidates for sensing various analytes are described in the next sub-section.

\subsection{Sensing}

6.2.1 Explosive sensing. With the advancement in science, the discovery of various hazardous compounds has resulted in many international and world wars being won. However, these nitro-based compounds such as TNP, TNT, RDX and explosive D pose a great threat to the ecosystem, human health and national/ international security due to their blasting nature. ${ }^{228,229}$ For some time, porous materials proven to be promising candidates to detect these explosive compounds in various media, particularly water. ${ }^{230} \mathrm{COFs}$ are one of the emerging candidates among these materials due to their potential and high emissive properties, large surface areas, pore size and pore volume, long-range order and thermal and chemical stability. ${ }^{231}$

In 2012, the first melamine-based porous organic framework, SNW-1, was efficiently synthesized by W. Zhang et al. from terephthaldehyde (TA) and melamine (MA) using the microwave-assisted technique. ${ }^{232}$ The synthesized nanoparticles possessed a highly cross-linked covalent-bonded network with a 
particle size of 20-50 nm and BET surface area of $476 \mathrm{~m}^{2} \mathrm{~g}^{-1}$. Due to its nano size, quite high porosity and significant emissive properties, the material was rapidly responsive for the selective detection of nitroaromatic explosives in THF-water $(10 \% \mathrm{v} / \mathrm{v})$ at ppb levels. The framework, being electron-rich, senses $\pi$-acidic nitroaromatics such as 2,4,6-trinitrophenol (TNP), 2,4,6-trinitrophenylmethylnitramine (tertyl), 2,4-dinitrotoluene (DNT), 2,4,6-trinitrotoluene (TNT), 4-nitrotoluene (NT), nitrobenzene (NB) and tends to exhibit strong quenching by the photo-induced electron transfer (PET) mechanism. TNP resulted in the highest fluorescence quenching $(>80 \%)$ in the solution phase with a very low detection limit ( $c a .11 .5 \mathrm{ppb}$ ). In addition to PET, the formation of a Meisenheimer complex resulting from hydrogen bonding between the phenolic group of TNP and the secondary amine linkages of SNW-1 caused the quenching. This was confirmed by the blue shift from $261 \mathrm{~nm}$ to $244 \mathrm{~nm}$ in the absorbance peak of the SNW-1 nanoparticles in the presence of TNP. Moreover, a detailed time-dependent vapour phase sensing experiment was conducted for DNT with a detection limit down to $9.8 \mathrm{ppb}$. The authors also tested common organic solvents such as dimethylformamide, dimethylsulfoxide, ethanol, acetonitrile, acetone, and benzene, where no significant effect on the emission intensity of the SNW-1 NPs was observed. The utility of this COF for sensing applications gave a great push to exploit COFs in this direction.

The next year, Jiang et al. reported a nitrogen-rich 2D azinelinked COF with the pyrene-core monomer TFPPy (see Section 4.2). ${ }^{152}$ The chemically robust azine linkage in the Py-azine COF was unaffected in various polar and non-polar organic solvents and did not get destroyed even in inorganic acid and base such as aqueous $\mathrm{HCl}$ and $\mathrm{NaOH}$, respectively. Moreover, the intense emitting property of the COF originates from the extended conjugation via azine linkages between the monomers and from the pyrene fluorescent tag. Therefore, the Py-azine COF was used as a distinctive chemosensing detector for various nitrobenzene derivatives such as 2,4,6-trinitrophenol (TNP), 2,4dinitrophenol (DNP), 2,4-dinitrotoluene (DNT), 2-nitrophenol
(NP), and 2-nitrotoluene (NT). Turn-off sensing up to $69 \%$ fluorescence quenching was observed for TNP with a detection limit of $70 \mathrm{ppm}$, whereas other analytes quenched only around 13\%. The hydrogen-bonding interactions between the TNP analyte and the azine units, which serve as the open docking sites at the framework edges, lead to a reduction in the emission intensity of the Py-azine COF.

Eventually, numerous COFs have been reported with electron-rich and fluorescent moieties, leading to the detection of nitroaromatic explosives, which are electron-deficient. Thus, a turn-off sensing is seen due to the electron transfer process. A unique example of turn-off and turn-on was reported in 2015 utilizing COF thin films, which enhanced the phenomenon of chemical sensing in comparison to bulk COFs. Two frameworks, TpBDH and TfpBDH, were explored for sensing applications in the bulk and exfoliated form, namely TpBDH and TfpBDH-CONs (Fig. 59). ${ }^{149}$

TpBDH-COFs and CONs do not show PL activity due to the restricted $\pi$-conjugation arising from enol-keto tautomerism. Conversely, there are no free $\mathrm{OH}$ groups present in $\mathrm{TfpBDH}-$ COFs and CONs, which limit their tautomerism, and therefore, they exhibit high FL intensity. Various nitroaromatics, such as TNP, TNT, 2-NP, DNT and DNP, were detected using CONs in both solution and dispersed phase showing quite opposite trends. In the solution phase, the FL intensity of TfpCON is quenched by $63 \%$ with TNP (detection limit of $5.4 \times 10^{-5} \mathrm{M}$ ), which is attributed to the electronic charge transfer from the $\pi$ acidic analyte (the picrate anion) to the rich electron cloud of the protonated nanosheet. On the contrary, 'turn-on' sensing behaviour was observed when the weak blue emission of a TfpBDH-CON-coated paper strip changed to a bright-yellow color upon the addition of TNP analyte. This was caused by the proton transfer from the phenolic group of the TNP analyte to the basic nitrogen atom of the imine linkage in the solid CONs.

A similar example of a polyimide covalent organic nanosheet was reported by Zhang et al. ${ }^{233}$ PI-CONs were comparatively more emissive than PI-COFs, which were synthesized via ultrasonic
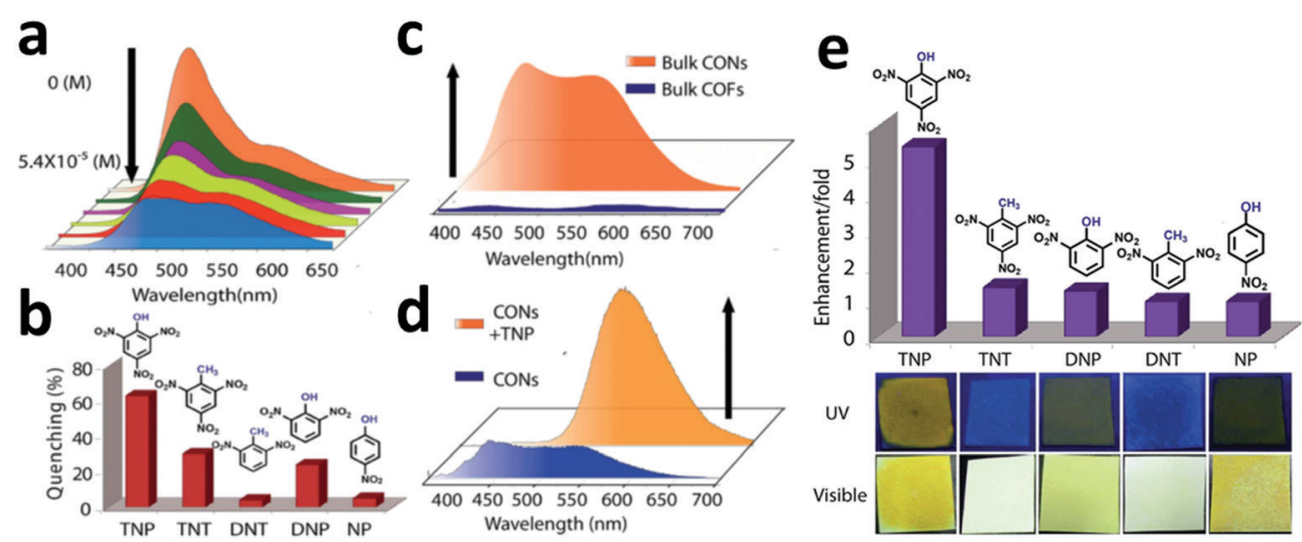

Fig. 59 (a) Turn off sensing (PL quenching) and (b) degree of fluorescence quenching of TfpBDH-CONs with different nitro analytes. (c) Photoluminescent spectra of bulk TfpBDH and TfpBDHCONs. (d) Photoluminescent spectra showing turn on (PL enhancement) of bulk TfpBDH-CONs and with TNP. (e) Photoluminescence enhancement plots of different analytes $\left[5.4 \times 10^{-3} \mathrm{M}\right]$ with TfpBDH-CONs. Reproduced with permission from ref. 149 , Copyright The Royal Society of Chemistry (2015). 
delamination. The nanosheet exhibited high quenching of TNP with $64 \%$ FL quenching and limit of detection $0.25 \mu \mathrm{M}$. The mechanistic explanation indicates electron transfer between the protonated PI-CONs and $\mathrm{TNP}^{-}$, which was supported by theoretical calculations using DFT functional and the B3LYP basis set, where upon its addition, the inner filter effect (IFE) could be seen. The IFE occurs when there is re-absorption of the emitted light by the quencher. The evident spectral overlap between the absorption spectrum of TNP and the emission spectrum of PI-CONs confirms this quenching phenomenon.

As presented in the previous sections, the controlled and systematic choice of the monomers and reaction conditions leads to a framework with the desired topology and dimensionality. A two-fold interpenetrated 3D-PY-COF emits yellow-green luminescence when dispersed in DMF and excited at $\lambda_{\text {ex }}=$ $408 \mathrm{~nm}$. The elevated FL intensity of the COF originates from the imine-functionalized pyrene moieties in its framework. ${ }^{46}$ The titration experiments evidently showed the selective and sensitive detection of TNP (up to 75\%) in DMF with a detection limit of $20 \mathrm{ppm}$. This was the first 3D-COF proven to be a potential candidate for sensing NACs.

Recently, Das et al. also reported a nitrogen-rich dualfunctionalized 2D COF, which was formed from triazine-based trialdehyde (tri(4-formylphenoxy)cyanurate) and electron-rich naphthalene diamine monomers. ${ }^{24}$ A unique six-fold interpenetrated framework was obtained, where the ABC stacked sheets gave rise to a star-like topology. The entangled hexagonal honeycomb sheets reduced the BET surface area $\left(c a .294 \mathrm{~m}^{2} \mathrm{~g}^{-1}\right)$, sculpting a triple-pore framework (ca. $0.75 \mathrm{~nm}, 1 \mathrm{~nm}$ and $2.1 \mathrm{~nm}$ ) corresponding to micro and mesopores. This multifaceted COF was utilized for (1) the detection of trace amounts of TNP in water due to the presence of $\pi$-electron-rich naphthalene moieties, (2) separation of benzene and cyclohexane using the $\pi$-acidic triazine moiety and (3) decoding of volatile organic compounds. Being highly emissive and chemically stable in aqueous media, the COF selectively detected the electron-deficient trinitrophenol up to the lower limit of $68 \mathrm{ppb}$ with a $K_{\mathrm{sv}}$ value 30-times higher than that for the other NACs. Further improvisation was reported for the ultrafast real-time detection of NACs through instant spot via paper strips. A significant color change from blue to green for the FL quenching by TNP was observed, making the COF very handy. This 'turn-off' sensing is attributed to the energy transfer from the framework to TNP analyte and photoinduced electron transfer from the LUMO of the COF to the lowlying conduction band of TNP. In addition, the H-bonded interactions between the $\mathrm{N}$ - and $\mathrm{O}$-functionalities and $-\mathrm{OH}$ group of TNP contributed $90 \%$ quenching.

Last year, another luminescent COF (namely ANCOF) was synthesized by Das et al. for the water-based sensing of nitroaromatic explosives and amines such as dichloronitroaniline (DCNA), 4-nitroaniline (4-NA), 2,4-dinitroaniline (DNA), 3-nitroaniline (3-NA), 2-nitroaniline (2-NA), aniline (AN), 4-nitrotoluene (4-NT), ethylene diamine (EDA), triethylamine (TEA), 1,2-diaminobenzene (DAB) and $N, N$-dimethyl-p-phenylenediamine (DMPD). ${ }^{154}$ The appended $\pi$-electron rich sites in the framework act as recognition sites for the selective detection of dichloran (other name for DCNA) and 4-NA in water/MeOH (Fig. 60). More than 80\% quenching with a large bathochromic shift in the FL intensity of ANCOF ( $c a$. $21 \mathrm{~nm}$ and $19 \mathrm{~nm}$ ) and lower limit of detection (ca. $142 \mathrm{ppb}$ and $89 \mathrm{ppb}$ ) for DCNA and 4-NA, respectively, was observed. The mechanistic insight showed the presence of resonance energy transfer, photo-induced electron transfer (PET) and distinctive $\mathrm{H}$-bonding interactions between the triazine moiety and $\mathrm{Cl}$ atom in DCNA and amine hydrogens in 4-NA. In addition, the lifetime measurements shed light on the static quenching mechanism at lower analyte concentrations, while the dynamic quenching mechanism occurs at higher concentrations. Moreover, the two-fold interpenetration of the framework with the $\mathrm{ABAB}$ arrangement yielded a beautiful marigold-like microfloral morphology.

6.2.2 pH sensing. The utility of nitrogen-rich luminescent COFs for developing $\mathrm{pH}$-sensing systems is an upcoming and barely explored area. Very few examples of COF-based fluorescent $\mathrm{pH}$ sensors have been reported in the literature. In 2016, the $\beta$-ketoenamine-based COF-JLU4 was the first to be explored as a pH-responsive material. ${ }^{144}$ The crystalline $2 \mathrm{D}$ framework a

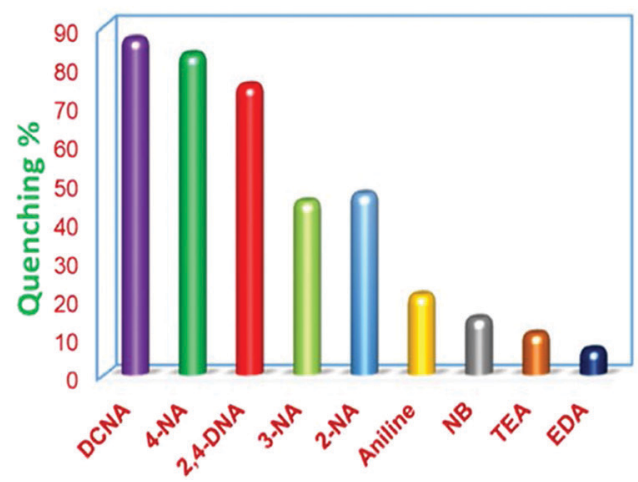

b

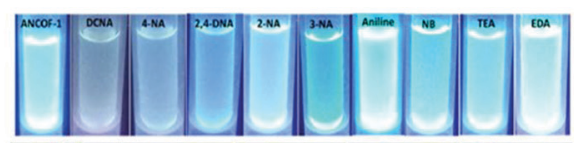

C

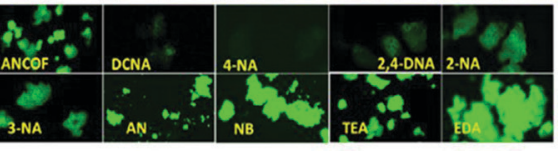

d

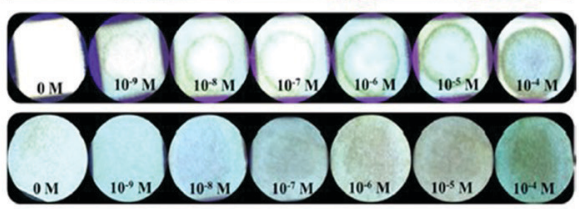

Fig. 60 (a) Percentage of fluorescence quenching obtained for different analytes at room temperature. (b) (top to bottom) Response of ANCOF in the presence of various amines by UV illumination, and (c) fluorescence microscopy images: response of ANCOF in the presence of various amine-analytes in the FITC region. Paper strips coated with ANCOF and different concentrations of (d) DCNA and (e) 4-NA. Reproduced with permission from ref. 154, Copyright American Chemical Society (2020). 
a
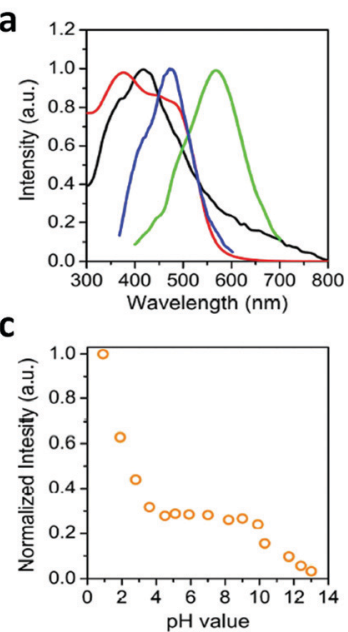

b

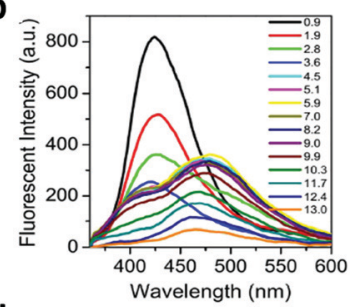

d

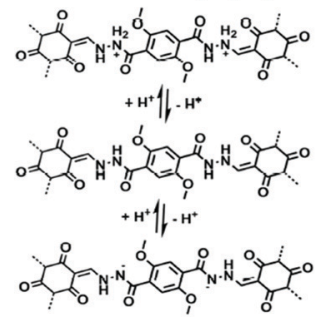

Fig. 61 (a) Electronic absorption (in water: black line and in the solid state: red line) and fluorescence spectra (in water: blue line and in the solid state: green line) of COF-JLU4. (b) pH-Dependent fluorescence of COF-JLU4 in aqueous solution with $\mathrm{pH}$ ranging from 0.9-13.0 measured under excitation at $334 \mathrm{~nm}$. (c) Photoluminescent intensity of COF-JLU4 at $428 \mathrm{~nm}$ in aqueous solutions with different $\mathrm{pH}$ values. (d) Deprotonation and protonation processes of the COF-JLU4 framework in acidic and basic media. Reproduced with permission from ref. 144, Copyright The Royal Society of Chemistry (2016).

showed excellent hydrolytic stability and wettability, a high BET surface area of $923 \mathrm{~m}^{2} \mathrm{~g}^{-1}$, large-sized pores with free $\mathrm{NH}$ groups, and good emissive properties. This makes it a pH-dependent fluorescence-responsive material for a wide range of $\mathrm{pH}$ in aqueous medium. It was observed that COFJLU4 exhibited the strongest fluorescent intensity in the most acidic conditions (Fig. 61). Going from pH 9.0 to 13.0 in the more basic region, the fluorescence intensity decreased gradually with the deprotonation of the $\mathrm{NH}$-groups present in the framework. Over the $\mathrm{pH}$ of 9.0 to 4.5 , not much change was observed in the luminescence intensity, but there was a blueshift in the emission peak at acidic $\mathrm{pH}$ 3.6. When the $\mathrm{pH}$ was below 3.6, the protonation of the NH-groups led to an enhancement of the peak at $428 \mathrm{~nm}$. This optical response of COF-JLU4 in a broad $\mathrm{pH}$ range is reversible and makes the COF a recyclable and reusable fluorescent $\mathrm{pH}$-sensor.

Another COF reported for colorimetric $\mathrm{pH}$ sensing contained a $\mathrm{pH}$-sensitive fluorescent chemical group, 8-hydroxyquinoline (HQ). ${ }^{234}$ COF-HQ was solvothermally synthesized by Chen et al. from the HQ-substituted terephthaldehyde monomer and TAPB, yielding a quite low surface area and semi-occupied pores. This HQ-functionalized framework possesses good acidic and hydrolytic stability, and thus exhibits high luminescent intensity, which is strongly correlated with the $\mathrm{pH}$ of the aqueous medium. Unlike COF-JLU4, the fluorescence intensity of COF-HQ is completely quenched in a highly acidic medium at $\mathrm{pH}=1.0$ due to the protonation of the 4-hydroxyquinoline group inside the pores. Conversely, when the $\mathrm{pH}$ is raised to 5.0, the HQ grafted inside the framework gets deprotonated, leading to a gradual increase in the emission intensity. This has a good linear relationship with the correlation coefficient of $R^{2}=0.979$.

COF-HQ acts as a colorimetric-based pH sensor as its color turns black when the luminescence is quenched and intensifies to dark orange under basic conditions at $\mathrm{pH} \geq 5.0$.

Recently, two porphyrin-based COFs were utilized as colorimetric $\mathrm{pH}$-responsive materials in the $\mathrm{pH}$ range of 0 to 7.0. TPE-Por4 and TPE-Por-2 were distinctively constructed under solvothermal conditions. $^{235}$ Between them, the highly crystalline TPE-Por-4 displays enhanced luminescence intensity due to (1) aggregationinduced emission (AIE) by the non-planar TPE chromophore, (2) the adequate layer spacing among the AB stacked COF, leading to the restriction of its intramolecular rotation (RIR), which enhances its FL emission, and (3) loose stacking and weak $\pi-\pi$ interaction because the twisted TPE prevents FL quenching. Furthermore, colorimetric pH-responsive studies were conducted, showing a bathochromic shift $(\sim 15 \mathrm{~nm})$ and hyperchromic shift of the emission peak from 2.01 to $4.15 \mathrm{~nm}$. This is due to the more protonated $\mathrm{N}$-atoms inside the porphyrin rings at a high hydrogen ion concentration, which destroys the $\pi$-electron-conjugated double-bond system, leading to fluorescence quenching. The TPE-Por-4 COF showed an obvious color change from dark red to bright green below pH 2.0, but no marked change above this value was seen.

Working in this direction, Das et al. reported the synthesis of three nitrogen-rich flexible COFs namely CON, CONN, and CONNCO (vide supra, Section 4.2) for sensing different acidic and basic environments. ${ }^{124}$ These flexible COFs are pH-stable, possess very high BET surface areas $\left(867 \mathrm{~m}^{2} \mathrm{~g}^{-1}\right)$ and pore size (4.2 $\AA$ for CONNCO), and have imine, amide, and hydrazide linkages, which provide inter and intramolecular $\mathrm{H}$-bonding among their stacked layers. These flexible COFs were utilized as reversible fluorescent $\mathrm{ON}-\mathrm{OFF} \mathrm{pH}$ sensing materials (Fig. 62). For CON, the luminescent intensity increases, and a sharp blue shift ( $\sim 50 \mathrm{~nm}$ ) was observed from $\mathrm{pH} 1.0$ to 13.0. In highly basic conditions, the deprotonation of the $\mathrm{N}$-atom leads to electron transfer towards the $\pi$-acidic triazine moiety, and hence a wavelength shift is observed in this region. On the contrary, CONN shows the opposite trend, exhibiting turn ON sensing from basic to acidic medium. This is due to the emergence of extra intramolecular H-bonding in acidic conditions together with the prior intermolecular $\mathrm{H}$-bonding between the carbonyl $\mathrm{O}$ and $\mathrm{NH}$ groups. Conversely, CONNCO exhibits the highest luminescent intensity at $\mathrm{pH} 4.0$ and shows moderate quenching at high acidic and basic conditions. Under these conditions, the intramolecular $\mathrm{H}$-bonding for CONNCO is destroyed, allowing intramolecular rotation, and the emission is quenched. From pH 7.0 to 4.0, the protonation of the $\mathrm{N}$-atoms in the hydrazide linkage dominate the intramolecular H-bonding, resulting in restriction of intramolecular rotations (RIR). The lifetime studies showed a dynamic process at basic $\mathrm{pH}$ for $\mathrm{CON}$ and at acidic $\mathrm{pH}$ for CONN, whereas at both conditions for CONNCO. The good agreement with the $R^{2}$ values, i.e., 0.982 and 0.984 in the polynomial fitting, and 0.999 in the linear fit for CON, CONN, and CONNCO, respectively, confirms the good correlation.

6.2.3. VOCs and small molecules. Recently, the potential of COFs as sensing probes has been explored for the detection of volatile organic and solvent molecules such as ammonia, ${ }^{29,236}$ 
(a)

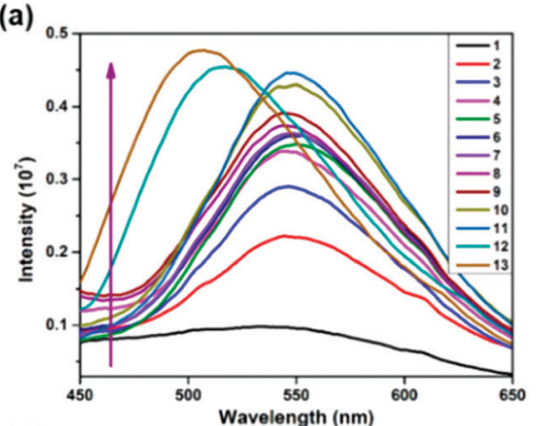

(d)

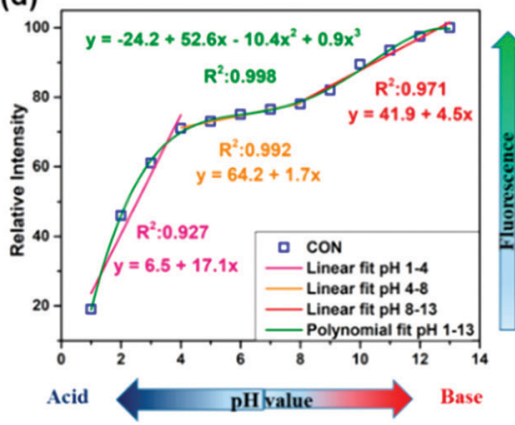

(b)

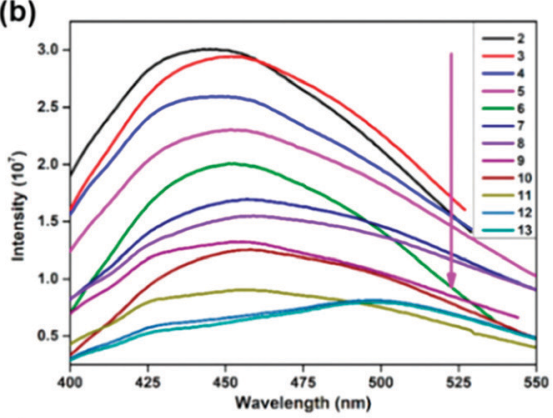

(e)

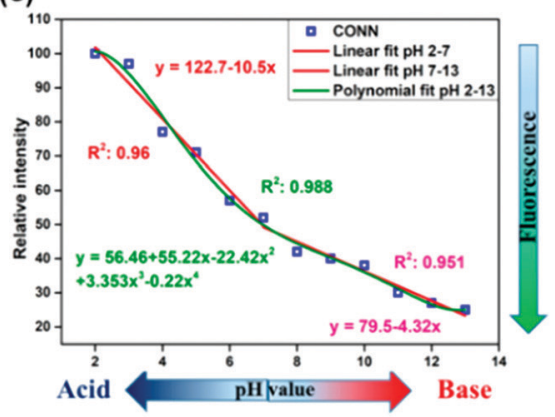

(c)

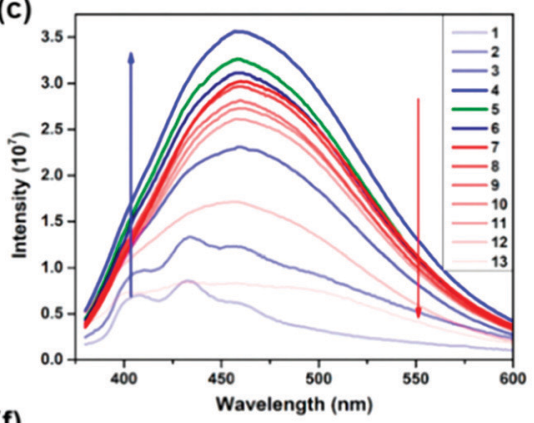

(f)

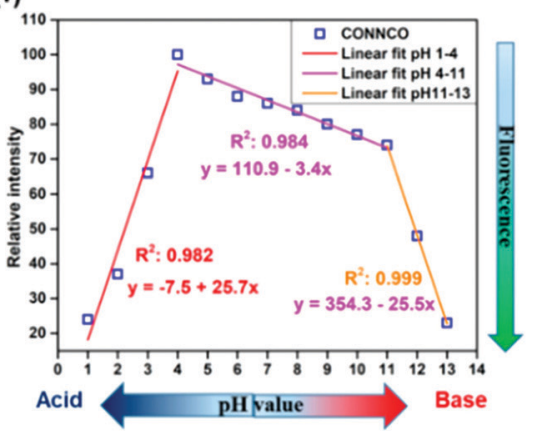

Fig. $62 \mathrm{pH}$-Dependent fluorescence spectra of (a) CON, (b) CONN, and (c) CONNCO in aqueous solution with pH ranging from 1 to 13. Relative fluorescence intensity of (d) CON, (e) CONN and (f) CONNCO at different pH. Reproduced with permission from ref. 124, Copyright the American Chemical Society (2021).

formaldehyde, ${ }^{237,238}$ arene, ${ }^{126}$ and $\mathrm{HCl}$ vapours, ${ }^{239,240}$ unsaturated aromatic volatile molecules ${ }^{24}$ and various protic and aprotic solvents. ${ }^{125,241}$ Several pesticides have also been detected using highly luminescent covalent organic frameworks. ${ }^{234}$

In 2016, Dalapati and co-workers reported the synthesis of TPE-Ph-COF, which is highly emissive due to aggregate-induced emission. ${ }^{29}$ The COF was synthesized via the solvothermal condensation of tetraphenylethene boronic acid (TPEBA) and 1,2,4,5-tetrahydroxybenzene (THB), developing boronate linkages. This COF was highly crystalline with a BET surface area of $962 \mathrm{~m}^{2} \mathrm{~g}^{-1}$, forming a dual-pore $\mathbf{k g m}$ net having pore sizes of 1.3 and $2.6 \mathrm{~nm}$. This material was utilized for the detection of ammonia molecules, which showed rapid quenching response in the organic solvents toluene and cyclohexane. It was explained that the Lewis acidic boronate linkages form acid-base interactions with the Lewis basic ammonia molecules, turning off the bright blue luminescence of the COF.

Xie $e t$ al. studied and reported the fluorescence properties of the highly crystalline TAT-COF-2. ${ }^{126}$ This $2 \mathrm{D}$ COF was synthesized from the $C_{3}$-symmetric trialdehyde and triamine derivative of triazatruxene (TAT), forming imine-linkages. The topological $\left[C_{3}+\right.$ $C_{3}$ ] connectivity weaves the COF with a high BET surface area and pore size of $1166.1 \mathrm{~m}^{2} \mathrm{~g}^{-1}$ and $1.48 \mathrm{~nm}$, respectively. Various electron-rich and electron-deficient arenes were successfully sensed using the strong electron-donating abilities of the TAT building unit (Fig. 63). It was found that the FL intensity was enhanced when an ethanolic suspension of TAT-COF-2 was diluted with electron-rich arenes such as toluene, chlorobenzene, $o$-dichlorobenzene, mesitylene, bromobenzene, 1,2-dimethylbenzene, pyridine, and some solvents such as $N$-methylpyrrolidone, tetrahydrofuran, ether, triethylamine, dimethylformamide, ethyl acetate, dimethylacetylamide, dimethyl sulfoxide, hexane and chloroform. On the contrary, significant fluorescence turn-off was seen upon dilution with nitrobenzene, 2-nitrotoluene, methanol, water, $n$-butanol, tetraethylene glycol, dioxane and phenol solution, which are electron-deficient species. Among them, total quenching was achieved upon the addition of 5 drops of nitrobenzene, while $\sim 60 \%$ enhancement was seen with toluene and mesitylene. The phenomenon of electron transfer was responsible for the whole quenching and enhancement of the emission peak.

Another truxene-based crystalline COF, Tx-COF-2, was reported recently by Nailwal $e t$ al., which was utilized for sensing vapor and solid-phase $\mathrm{HCl}^{240}$ The framework, being highly stable in $12 \mathrm{M}$ $\mathrm{HCl}$, was exposed to $\mathrm{HCl}$ vapor in a 1,4-dioxane suspension. An evident rapid color change from yellow to bright orange was seen, attributing to the protonation of the imine linkages in the framework, showing fast fluorescence enhancement. The detection limit was found to be as low as $4.5 \mathrm{nmol} \mathrm{L}^{-1}$, which is the best reported value for the detection of $\mathrm{HCl}$ vapor to date. The reversibility of the framework was noted up to 10 cycles using ammonia vapor with recovery of the framework using triethylamine when the $\mathrm{pH}$ is maintained at 7.

N-Rich frameworks with high emissive properties and crystallinity were reported by Mandal and co-workers in $2018 .{ }^{24} \mathrm{~A}$ dualfunctionalized $\mathrm{COF}$ was proven to be potent for sensing various tropospheric VOCs such as benzene, toluene, $o-, m$-, $p$-xylene, mesitylene, chloro-, bromo-, iodo-benzene, anisole and cyanobenzene. VOCs have great affinity towards electron-deficient centers, making the COF suitable for VOC sensing. The results showed that the host-guest charge transfer causes the formation of an exciplex, 


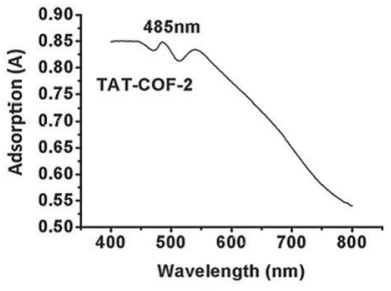

a)

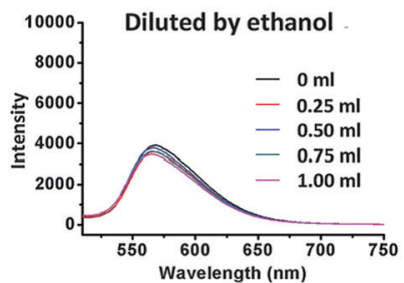

c)

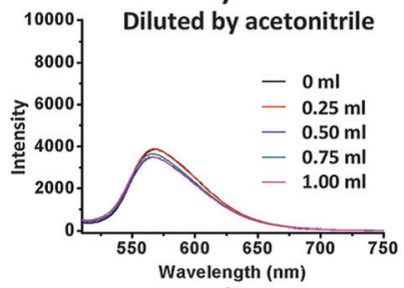

e)

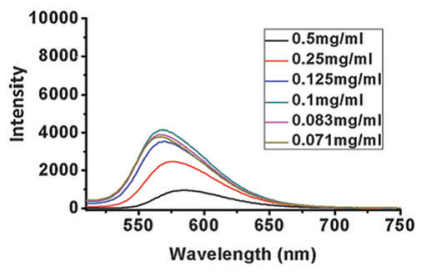

b)

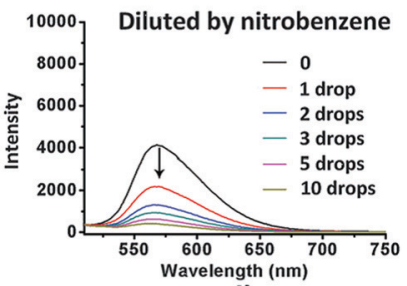

d)

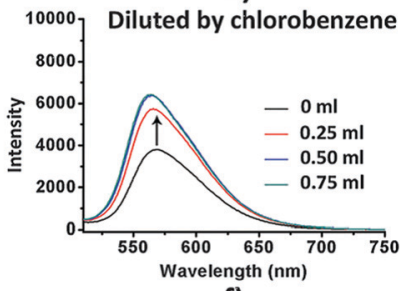

f)
Fig. 63 (a) UV-Vis adsorption of TAT-COF-2, (b) fluorescence of TATCOF-2 suspended in ethanol at different densities; $100 \mathrm{mg}$ TAT-COF-2 suspended in $1 \mathrm{~mL}$ ethanol and diluted with (c) ethanol, (d) nitrobenzene, with the calculated detection limit of about $10 \mathrm{mM}$; (e) acetonitrile and (f) chlorobenzene, with the calculated detection limit of about $198 \mathrm{mM}$. Reproduced with permission from ref. 126, Copyright The Royal Society of Chemistry (2015).

changing the FL intensity and emission wavelength in each case. COF $\supset$ mesitylene showed the maximum emission intensity at $381 \mathrm{~nm}$ with a high quantum yield ( $c a$. 0.083) and long average lifetime ( $c a .3 .89 \mathrm{~ns}$ ), which was blue-shifted in comparison to other electron-donating VOCs. Conversely, there was a red-shift in the emission wavelength of the COF when benzene was substituted with a heavy atom, viz., iodobenzene, with an increase in its average lifetime. Moreover, the same triazine core was utilized to sense and separate aromatic benzene $(\mathrm{Bz})$ molecules from the non-aromatic cyclohexane (Cy) domain. A red shift in the luminescence intensity of the COF was observed when immersed in benzene with quantum yields of $0.06 / 0.042$ and average lifetimes of 3.47/4.02 ns (Bz/Cy), respectively. This is ascribed to the very strong interactions between the electron-rich $\mathrm{Bz}$ and electron-deficient triazine core in the framework.

In recent years, it has been explored that covalent organic frameworks provide a site for hydrogen bond interactions with molecules having carbonyl groups. This led to the sensing of small molecules such as formaldehyde, where the electronegative oxygen atom from the carbonyl group forms hydrogen bonds with the aromatic hydrogens present in the framework. Hao and co-workers investigated the effect of the luminescent properties of the HHTP-DPB COF before and after sensing formaldehyde molecules. ${ }^{237}$ It was observed that the FL intensity of the HHTPDPB COF was rapidly quenched after the incremental addition of formaldehyde. Employing the quantum chemical approach, DFT
AND TDDFT methods, it was determined that the H-bonding is enhanced in the $S_{1}$ state, leading to a diminished energy gap between G. S. and E. S. by $0.54 \mathrm{eV}$ after the interaction of formaldehyde and the COF. This was further confirmed by calculation of the H-bond lengths, IR spectra of the donor and acceptor groups and NMR chemical shifts for the framework hydrogens involved in $\mathrm{H}$-bonding in the $\mathrm{S}_{0}$ and $\mathrm{S}_{1}$ states. Also, the FL rate coefficients for the COF and HCHO@COF were $1.22 \times 10^{4}$ and $4.13 \times 10^{3} \mathrm{~s}^{-1}$, respectively, indicating that the FL intensity weakened after the addition of the analyte.

Similar studies were carried out with TT-COF reported by Hussain et al. ${ }^{238}$ The noncovalent interactions between the thienothiophene-based COF and formaldehyde were investigated using DFT and TDDFT computational tools, which are indicative of strengthened $\mathrm{H}$-bonding and weakened emission intensity after mixing. The FL rate constants for the simulated TT-COF and its complex with HCHO are $1.16 \times 10^{8}$ and $1.45 \times$ $10^{3} \mathrm{~s}^{-1}$, respectively, strongly indicating the quenching effect. Furthermore, the reduction in band gap between the $S_{1}$ and $T_{1}$ states $(1.00$ to $0.32 \mathrm{eV})$ indicates the possibility of a radiationless ISC process as the reason for quenching.

Recently, Mandal and co-workers reported two highly crystalline, luminescent and chemically stable COFs, COF-N and COF-NN, decorated with molecular docking sites for solvatochromic decoding of volatile solvent molecules (VSMs). ${ }^{125}$ The COFs possess pore sizes of 2.7 and $3.06 \mathrm{~nm}$, respectively, with moderate BET surface areas, and thus, the structurally small molecules were deciphered by these materials. On immersing the two COFs in different VSMs with varying polarities such as alcoholic $(\mathrm{MeOH}, \mathrm{EtOH}$, 1-PrOH, 2-PrOH, 1-BuOH, 2-BuOH and $t-\mathrm{BuOH}$ ), chlorinated (DCE, $\mathrm{CHCl}_{3}$, and $\mathrm{CH}_{2} \mathrm{Cl}_{2}$ ) and some aprotic solvents (acetone, acetonitrile, THF, dioxane, EtOAc, DMF, DMA and DMSO), a remarkable color change together with a change in wavelength and emission intensity was noted (Fig. 64). The interactions between the COFs and VSMs were examined using polarity parameters such as Reichardt's, Kamlet-Taft's and Gutmann's parameters. The results showed that the singlet energies are in good luminescence correlation with Reichardt's parameters for COF-N and that with Gutmann's parameters for COF-NN. Moreover, the presence of an extra amine functionality in the COF-NN framework contributed to the electron donation and enhanced the $\mathrm{H}$-bonding interactions with the VSMs.

6.2.4 Toxic anions. The sensing of toxic acidic radicals in various solvents has been practiced for some time. N-rich COFs have been extensively utilized for this purpose due to their porous nature, exceptional stability and availability of reversible proton donor/acceptor sites in their framework.

Considering that pyrene-core organic moieties have been widely explored for AIE in covalent organic frameworks, they have also been utilized for turn-on sensing of anionic molecules. An efficient but weakly luminescent N-rich TFPPy-DETHz COF was fabricated through hydrazone bridges and emitted low green-yellow FL intensity due to the ACQ effect (vide Section 6.1.2). ${ }^{137}$ The luminescent intensity of the COF was enhanced by 3.8-fold that of its original intensity after the addition of a THF solution of tetrabutylammonium fluoride to it. On the 
(a)

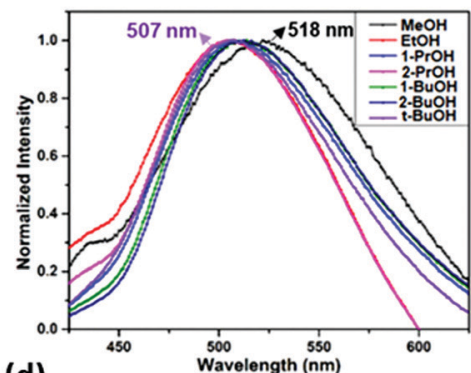

(b)

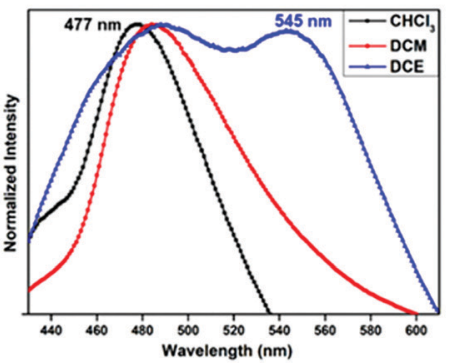

(c)

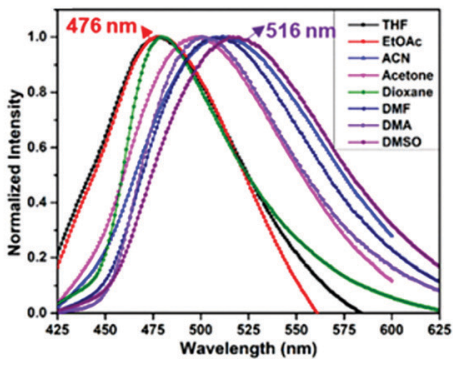

(d)

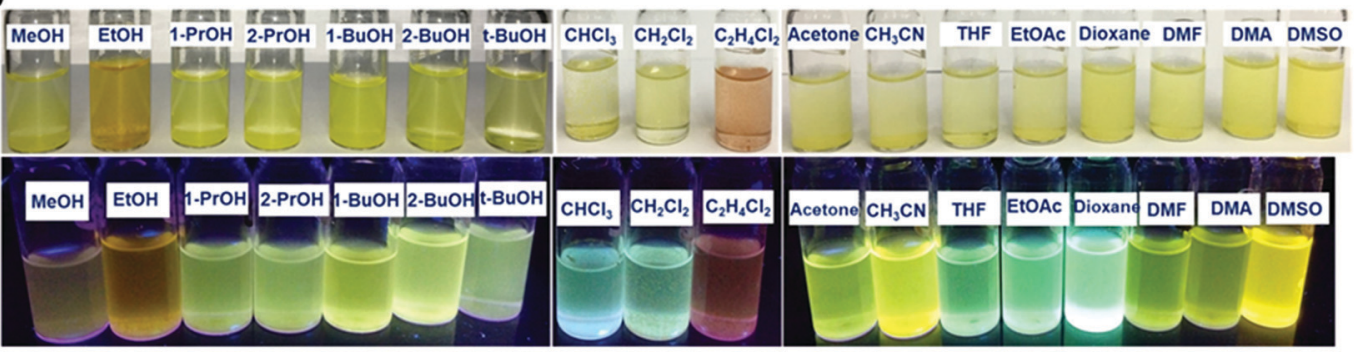

(e)

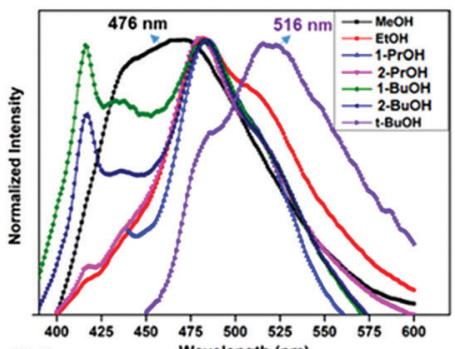

(f)

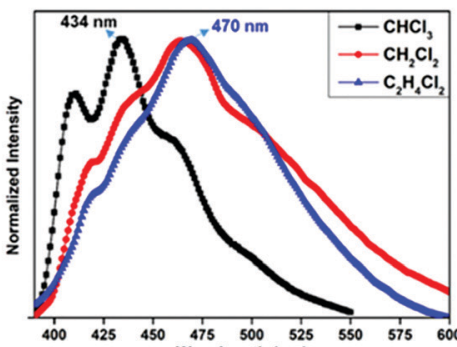

(g)

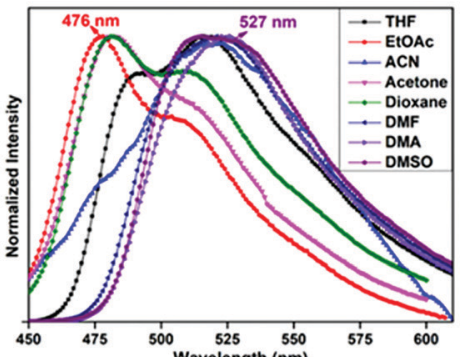

(h)

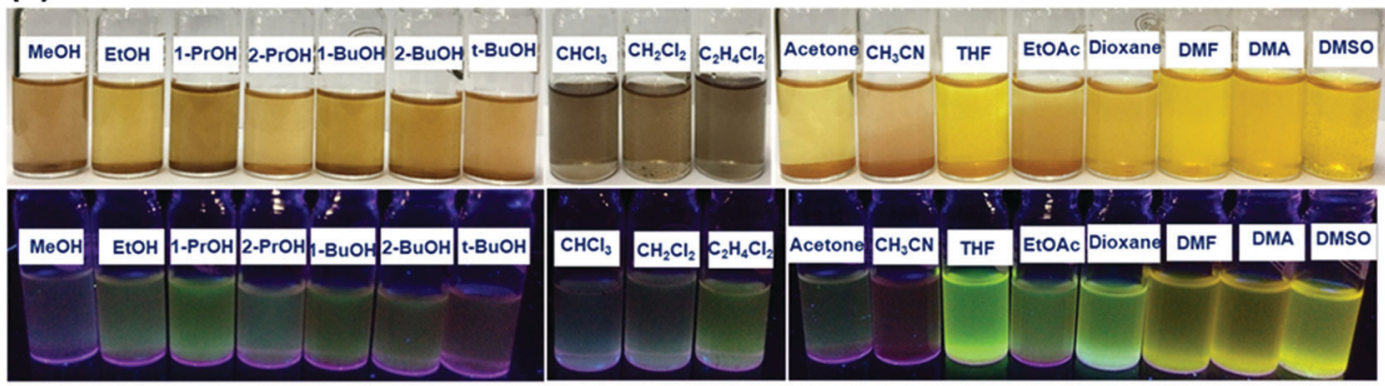

Fig. 64 Emission spectra of COF-N and COF-NN in different ( $a$ and e) alcoholic, (b and f) chlorinated, and (c and g) aprotic VSMs (0.25 mg in 2 mL). (d and h) Solvatochromic behavior of COF-N and COF-NN in the presence different VSMs (top row: under visible light and bottom row: UV illumination). Reproduced with permission from ref. 125, Copyright the American Chemical Society (2020).

contrary, other halogen and nitrate ions did not have any effect on the weakly luminescent framework. This was ascribed to the strong acid-base reaction between the $\mathrm{F}^{-}$ions and $\mathrm{N}-\mathrm{H}$ units of the hydrazone linkages, which led to deprotonation of these units. However, reversible protonation of $\mathrm{N}^{-}$anions can be successfully carried out by adding triflic acid (TFA) to the system. It must be noted that the TFPPy-DETHz COF was the first report of anion sensing using a porous organic framework.

Common oxidizing agents, such as $\mathrm{CrO}_{4}{ }^{2-}, \mathrm{Cr}_{2} \mathrm{O}_{7}{ }^{2-}$ and $\mathrm{MnO}_{4}{ }^{-}$, are widely used in laboratories and industry, which pose a threat to the environment and mankind. Therefore, it is essential to monitor and sense these anions using efficient and easy to handle probes, such as porous organic polymers. Li and co-workers developed the 3D stable and luminescent COF-TT, which exhibited high sensitivity towards the detection of $\mathrm{CrO}_{4}{ }^{2-}$, $\mathrm{Cr}_{2} \mathrm{O}_{7}{ }^{2-}$ and $\mathrm{MnO}_{4}{ }^{-}$ions via a turn-off sensing phenomenon (Fig. 65a). ${ }^{242}$ The COF-TT framework contains a flexible core synthesized from bis(tetraoxacalix[2]arene[2]triazine) and the rigid tetra(paminophenyl)methane. When anions interact with the framework in aqueous medium, a strong competitive energy transfer process occurs, which was validated by UV-Vis spectroscopy. The spectral overlap of the adsorption band of COF-TT with the wide range absorption bands of the three anions supports the quenching mechanism. With up to $0.8 \mathrm{mM}$ 
a

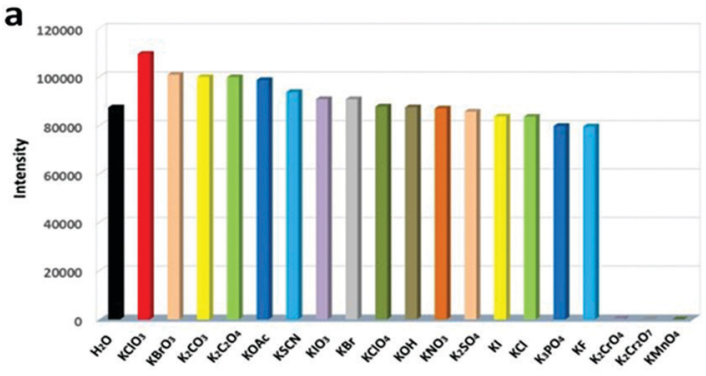

b

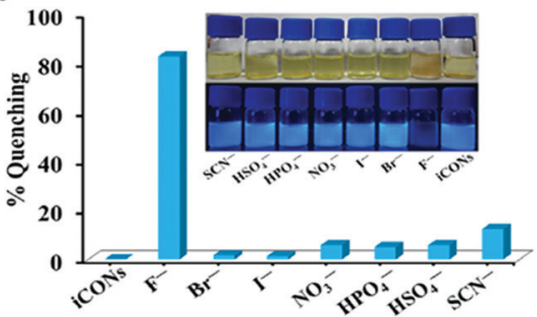

Fig. 65 (a) Fluorescence intensities of COF-TT for different anions. (b) \% quenching of DATGCl-iCONs upon the addition of different anions. Reproduced with permission from: (a) ref. 242, Copyright The Royal Society of Chemistry (2019) and (b) ref. 114, Copyright the American Chemical Society (2020).

(for $\mathrm{CrO}_{4}{ }^{2-}$ and $\mathrm{Cr}_{2} \mathrm{O}_{7}{ }^{2-}$ ) and $0.7 \mathrm{mM}$ (for $\mathrm{MnO}_{4}{ }^{-}$) anionic concentration, the framework completely lost its luminescent property. This makes COF-TT an excellent anion sensor with a detection limit of $3.43 \times 10^{-4} \mathrm{M}$ (for $\mathrm{CrO}_{4}{ }^{2-}$ and $\mathrm{Cr}_{2} \mathrm{O}_{7}{ }^{2-}$ ) and $3.20 \times 10^{-4} \mathrm{M}$ (for $\mathrm{MnO}_{4}{ }^{-}$).

Another strategy for anion sensing is by incorporating an ionic linker as a monomeric unit to synthesize ionic frameworks. Recently, triguanidinium chloride $\left(\mathrm{TG}_{\mathrm{Cl}}\right)$ was utilized as an anion acceptor linker, which was combined with a fluorescent-active dimethoxyterephthaldehyde (Da) unit to give ionic covalent organic nanosheets, namely $\mathrm{DATG}_{\mathrm{Cl}}$-iCONs, via self-exfoliation in methanol (Fig. 65b). ${ }^{114}$ The addition of $\mathrm{F}^{-}$to the cationic sensor caused great fluorescence quenching with a detection limit of $5 \mathrm{ppb}$ level. A unique property of the guanidinium linker is its ability to donate a proton (in the presence of $\mathrm{F}^{-}$ions) and abstract it again (by adding $\mathrm{HCl}$ ). The appropriate basic nature of the $\mathrm{F}^{-}$ions in the framework environment leads to the abstraction of a proton, which gives rise to turn-off sensing. Moreover, the further addition of $\mathrm{HCl}$ supplies enough $\mathrm{H}^{+}$to bind the guanidinium unit and regain its fluorescent property. According to the theoretical studies, it was found that the quenched luminescence of $\mathrm{DATG}_{\mathrm{Cl}}$-iCONs occurs via the photo-induced electron transfer mechanism, which was supported by time-resolved fluorescence spectroscopy, showing dynamic quenching. The turn-off/on sensing was repeated successfully for up to five cycles with minimum loss of the sensor.

6.2.5 Metal ions. The increasing amount of metal ions in water bodies poses a huge threat to the ecosystem and mankind. This global concern has attracted great attention toward the development of novel strategies for monitoring and detecting heavy metal ions. ${ }^{243,244}$ The first example in this direction was reported in 2016, where an azine-linked COF, namely COF-JLU3, was explored to detect $\mathrm{Cu}^{2+}$ ions in THF medium. ${ }^{245}$ This COF exhibits strong luminescence at $601 \mathrm{~nm}$ in the orange-red region upon excitation at $424 \mathrm{~nm}$ (Fig. 66a). The framework contains high porosity, surface area and rich hetero-atom content, such as $\mathrm{N}$ and $\mathrm{O}$, which act as Lewis basic sites for ion sensing. Among the various metal nitrates, $\mathrm{Cu}^{2+}$ ions were selectively detected, and the FL intensity of the framework was reduced to $18.3 \%$ when 2.0 equivalents of $\mathrm{Cu}\left(\mathrm{NO}_{3}\right)_{2}$ was dispersed with COF-JLU3 in THF solution. The selective detection of cuprous ions is ascribed to the partly filled d-orbitals in comparison to alkaline metals (with no d-subshells) and $\mathrm{d}^{10}$ transition metal ions. Among $\mathrm{Fe}^{3+}, \mathrm{Co}^{2+}$ and $\mathrm{Cu}^{2+}$ ions, the highest selectivity of the framework was found with $\mathrm{Cu}^{2+}$ ions with the detection limit of $0.31 \mu \mathrm{M}$ and Stern-Volmer constant of $3.8 \times 10^{4} \mathrm{M}$. The Lewis acid-base interactions are mainly responsible for this quenching due to photo-induced electron transfer (PET) from the COF to metal ion. The time-resolved lifetime measurements indicated the FL lifetime for COF-JLU3 was reduced from $1.5 \mathrm{~ns}$ to $0.7 \mathrm{~ns}$ when dispersed with $2 \times$ $10^{-4} \mathrm{M} \mathrm{Cu}^{2+}$ nitrate solution.

Other COFs for the efficient sensing of $\mathrm{Cu}^{2+}$ ions are Q-graphene (QG)-grafted COF ${ }^{246}$ which detects up to $0.5 \mathrm{nM}$, and nitrogen-rich $\mathrm{CTF}^{247}$ with a detection limit of $0.05 \mu \mathrm{gL}^{-1}$.

Bipyridine-based COF nanosheets (Tp-Bpy NSs) exhibit a structure with regular pores and abundant $\mathrm{N}$-based moieties, which act as active sites for the detection of $\mathrm{Hg}^{2+}$ ions. ${ }^{248}$ The Tp-Bpy nanosheets were prepared via ultrasonic exfoliation, on which Au nanoparticles were loaded to form the AuNPs@Tp-Bpy nanocomposite. The affinity between gold and mercury suggests the high selectivity of AuNPs@Tp-Bpy towards $\mathrm{Hg}^{2+}$ ions with a detection limit of $0.33 \mathrm{nM}$. The framework was enriched with nitrogen, which provides ultra-affinity for Au nanoparticles, and therefore, it provides an excellent platform to detect $\mathrm{Hg}^{2+}$ with good selectivity and sensitivity in real-life sensing applications.

In addition, COF-LZU8 was also utilized for sensing $\mathrm{Hg}^{2+}$ with a very low detection limit of $25.0 \mathrm{ppb} .^{249}$ Recently, COFs have been explored to make quantum dots, which exhibit high luminescent properties for metal ion analyte sensing. The CTF-based N-doped quantum dots (CTFQDs) detected $\mathrm{Hg}^{2+}$ ions up to a limit of $0.23 \mu \mathrm{M}$ (Fig. 66b). ${ }^{250}$

The highly fluorescent AH-COF, which was synthesized using hydroxyl functionalized 2,5-diallyl-3,6-dihydroxyterephthalohydrazide and 1,3,5-benzenetricarboxaldehyde, was utilized for selective and rapid $\mathrm{Hg}^{2+}$ ion sensing (Fig. 66c). ${ }^{251}$ This COF possesses exceptional luminescent properties due to its excitedstate intramolecular proton transfer (ESIPT) and increased $\pi$-conjugation. According to the simulated PXRD showing its eclipsed packed structure, one unit cell exhibits six reacting receptor sites, which consist of allyl and hydroxyl groups. The $\mathrm{Hg}^{2+}$ ions firstly bind to the hydroxyl groups, forming an $\mathrm{Hg}$-containing five-membered ring, blocking the ESIPT mechanism from occurring, which finally quenches the FL intensity of the framework.

For the detection of toxic lead ions in aqueous media, a novel electrochemical sensor, named TAPB-DMTP-COF, was utilized. $^{252}$ This COF was formed via the imine condensation 
of 1,3,5-tris(4-aminophenyl)benzene (TAPB) and 2,5-dimethoxy terephthaldehyde (DMTP). Lead ions were attached to the surface of TAPBDMTP-COF via the formation of a complex with its amine moiety. This sensor exhibited a broad linear range, ultra-stability, low detection limit and great sensitivity towards $\mathrm{Pb}^{2+}$ ions due to its large active sites and surface area. The wide surface area of these electrochemical sensors will provide an excellent platform in environmental monitoring and clinical diagnostic applications.

A series of imine-linked COFs, namely TaTa, DhaTab, TRITER-1 and TzDa, was utilized for the detection of $\mathrm{Fe}^{3+}$ ions in $\mathrm{DMF} / \mathrm{H}_{2} \mathrm{O}(9: 1, \mathrm{v} / \mathrm{v})$ medium. The luminescent-active $\mathrm{N}$-rich frameworks underwent strong PL quenching due to the absorption competition quenching effect of $\mathrm{Fe}^{3+}$ ions, with the highest quenching coefficient for DhaTab $\left(1.0 \times 10^{5} \mathrm{M}^{-1}\right.$, LOD = $0.12 \mu \mathrm{M})$ followed by TaTa $\left(1.4 \times 10^{4} \mathrm{M}^{-1}\right)$, TRITER- $1(5.6 \times$ $\left.10^{3} \mathrm{M}^{-1}\right)$ and TzDa $\left(3.3 \times 10^{3} \mathrm{M}^{-1}\right)$. Moreover, a slight increase in the luminescent intensity of all four COF sensors was observed due to their strong interactions with $\mathrm{Al}^{3+}$ ions. ${ }^{253}$ In the following year, a new hydrazone-linked Bth-Dma COF was employed as a sensor to detect $\mathrm{Fe}^{3+}$ ions via the selective coordination of these ions with the $\mathrm{O}, \mathrm{N}, \mathrm{O}^{\prime}$-chelating sites on the pores of the framework (vide supra, Section 4.2). ${ }^{141}$ Switch-off quenching was reported with an LoD value of $0.17 \mu \mathrm{M}$. Additionally, neither the presence of anions $\left(\mathrm{Cl}^{-}, \mathrm{Br}^{-}, \mathrm{NO}_{3}{ }^{-}\right.$and $\left.\mathrm{OAc}^{-}\right)$nor change in $\mathrm{pH}$ from 4 to 10 caused a change in the quenching profile of the COF (Fig. 66d). To support the electron and energy transfer quenching mechanism,
XPS was used, which showed a decrease in the binding energy values for Bth-Dma and Bth-Dma@Fe ${ }^{3+}$ (see Section 5.5). Later, Asadi et al. synthesised benzimidazole-linked COFs, viz. COF-BI-1 and COF-BI-2, which were conventionally synthesized using pyromellitic dianhydride (PMDA)/Perlin-tetracarboxylic anhydride (PTCA), 5-aminoisophthalic acid and 3,3'-diaminobenzidine, showing significant fluorescent quenching with the incremental addition of an $\mathrm{Fe}^{3+}$ ion solution to COF-BI-1 in THF and COF-BI-2 in DMF up to a limit of $1.3 \mu \mathrm{M}^{254}$

An evident fluorescence "turn-on" mechanism was reported by Qiu and co-workers, where the PL intensity of the waterstable covalent organic nanosheets Bpy-NSs was enhanced with the incremental addition of $\mathrm{Al}^{3+}$ ions among several monovalent $\left(\mathrm{Li}^{+}, \mathrm{Na}^{+}, \mathrm{K}^{+}\right.$, and $\left.\mathrm{Ag}^{+}\right)$, divalent $\left(\mathrm{Zn}^{2+}, \mathrm{Mn}^{2+}, \mathrm{Cd}^{2+}, \mathrm{Cu}^{2+}, \mathrm{Mn}^{2+}\right.$, $\mathrm{Ni}^{2+}, \mathrm{Co}^{2+}, \mathrm{Pb}^{2+}, \mathrm{Ba}^{2+}$, and $\left.\mathrm{Ca}^{2+}\right)$ and trivalent $\left(\mathrm{Cr}^{3+}\right.$ and $\left.\mathrm{Fe}^{3+}\right)$ metal ions in aqueous media. ${ }^{255}$ The photoluminescence intensity of the N-rich Bpy-NSs nanosheets was limited due to extensive energy dissipation by their bipyridine (Bpy) moieties, leading to photoinduced electron transfer to the phloroglucinol centres. However, the addition of $\mathrm{Al}^{3+}$ ions resulted in the formation of a strong Lewis acid-base coordination complex with the Bpy-core, in which the electrons occupy the lowest energy orbital, preventing the PET process. The detection limit for $\mathrm{Al}^{3+}$ ions was as low as $0.33 \mu \mathrm{M}$.

The olefin-linked TFPT-BTAN-AO COF was formed via Knoevenagel condensation followed by post-synthetic modification through amidoximation by treating it with excess $\mathrm{NH}_{2} \mathrm{OH} \cdot \mathrm{HCl}$ at

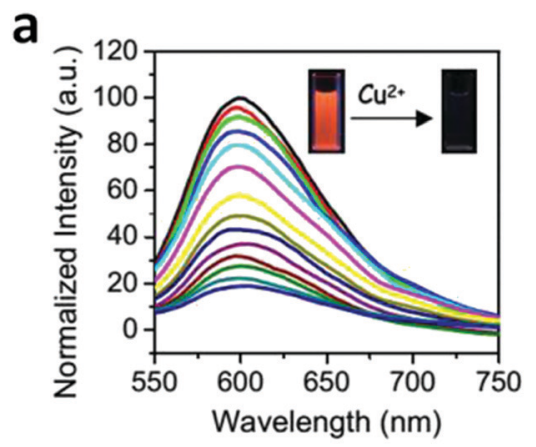

b

C

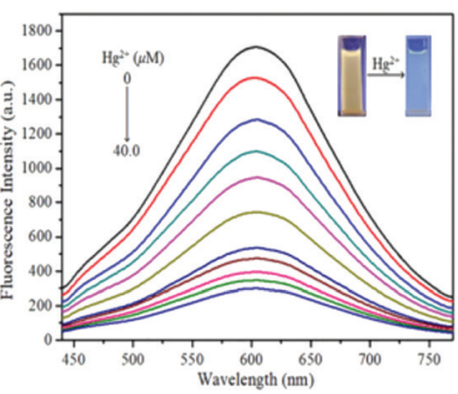

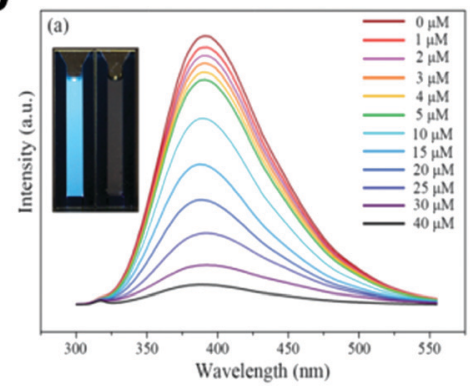

d

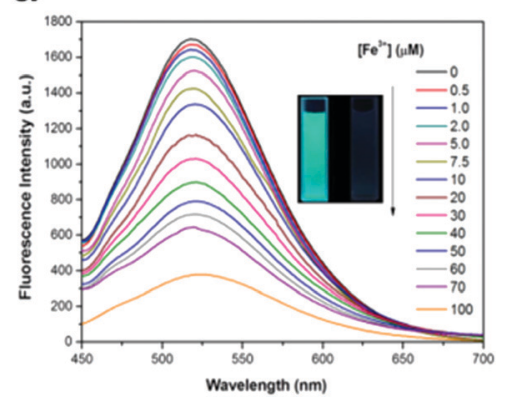

Fig. 66 (a) Luminescence spectra of COF-JLU3 in THF suspension containing different concentrations of $\mathrm{Cu}^{2+}\left(0\right.$ to $\left.2 \times 10^{-4} \mathrm{M}\right)$, $\lambda_{\mathrm{ex}}=400 \mathrm{~nm}$. The inset shows the corresponding images of COF-JLU3 under UV irradiation at $365 \mathrm{~nm}$ before and after titration with $\mathrm{Cu}^{2+}$ ions. (b) Fluorescence emission spectra of CTFQD in aqueous solution at different concentrations of $\mathrm{Hg}^{2+}$. (c) Fluorescence titration of $\mathrm{AH}-\mathrm{COF}$ in the acetonitrile upon treatment with $\mathrm{Hg}^{2+}$. (d) Fluorescence emission spectra of Bth-Dma COF at various concentrations of $\mathrm{Fe}^{3+}$ ions. Reproduced with permission from: (a) ref. 245, Copyright The Royal Society of Chemistry (2016); (b) ref. 250, Copyright The Royal Society of Chemistry (2017); (c) ref. 251, Copyright Elsevier (2020) and (d) ref. 141, Copyright the American Chemical Society (2019). 


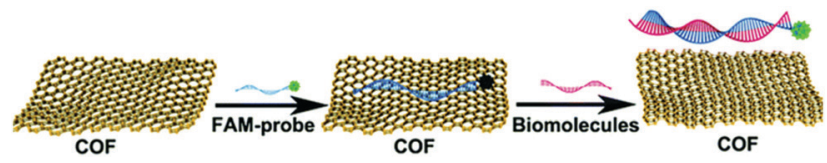

Fig. 67 Turn-on fluorescent sensor for the detection of biomolecules. Reproduced with permission from ref. 257, Copyright The Royal Society of Chemistry (2017).

$85{ }^{\circ} \mathrm{C}$ for 24 h. ${ }^{256}$ This made the framework rich in $-\mathrm{OH}$ and $-\mathrm{NH}_{2}$ functional groups and the material was utilized to detect uranium ions in aqueous medium. The $\mathrm{UO}_{2}{ }^{2+}$ units reversibly bind to the $\mathrm{N}$ and $\mathrm{O}$ atoms of the amino and the hydroxyl groups, respectively, and the COF detects uranium ions up to $6.7 \mathrm{nM}$ with an ultra-fast response time of 2 s. The $\mathrm{UO}_{2}{ }^{2+}$ @TFPT-BTAN-AO COF could be regenerated when dispersed in $1 \mathrm{M} \mathrm{Na} \mathrm{NO}_{3}$ aqueous solution as it has $>95 \%$ elution efficiency for up to 6 cycles.

6.2.6 Bioanalytes. Functionalized porous COFs behave as potent biosensors if they contain the biological receptor elements and mimic biological species for their sensing in a suitable environment.

In 2017, Li et al. reported a biosensor, named TpTta-COF, which was solvothermally synthesized using $4,4^{\prime}, 4^{\prime \prime}$-(1,3,5-triazine-2,4,6triyl)trianiline (Tta) and 1,3,5-triformylphloroglucinol (Tp) units. ${ }^{257}$ Initially, the COF was tagged with an FAM-labeled probe, which was adsorbed on its surface and inside its pores via $\mathrm{H}$-bonding and $\pi-\pi$ stacking interactions (Fig. 67). The carboxyfluorescein in the FAM probe shows very strong luminescence, which was quenched upon contact with COF (acceptor) via fluorescence resonance energy transfer (FRET). The probe ( $5^{\prime}$-FAM-TCG CTA ACC TGT TCA TCG TAG-3') was uniquely designed to be complementary to the target DNA analyte (5'-CTA CGA TGA ACA GGT TAG CGA-3'). After the DNA analyte comes in contact with the COF quenched FAM-labeled probe, the formation of double-stranded DNA occurs, resulting in complete detachment of the probe from the framework. The rapid formation and detachment process resulted in the turn-on sensing of DNA with the limit of $3.7 \mathrm{nM}$.

Peng et al. reported the synthesis of imine-based covalent organic nanosheets (NS), TPA-COF NSs, via the chemical exfoliation of TPA-COF, which was previously synthesized using tris(4formylphenyl)amine (TFPA) and tris(4-aminophenyl)amine (TAPA)
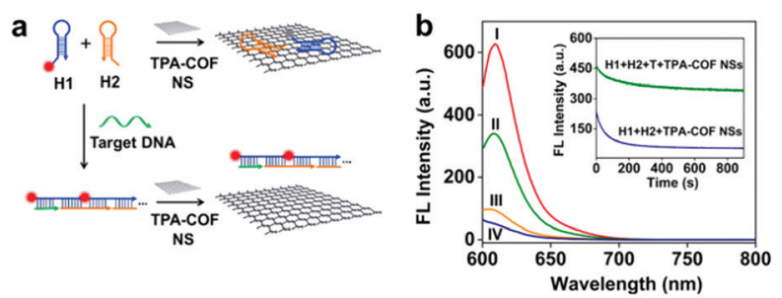

Fig. 68 (a) Schematic representation of a TPA-COF NS-based fluorescence sensor to detect DNA. (b) Fluorescence spectra recorded with different conditions: (I) $\mathrm{H} 1+\mathrm{H}_{2}$; (II) $\mathrm{H} 1+\mathrm{H} 2+\mathrm{T}+\mathrm{TPA}-\mathrm{COF} N \mathrm{NS}$; (III) $\mathrm{H} 1+\mathrm{T}+\mathrm{TPA}-\mathrm{COF}$ NSs; and (IV) H1 + H2 + TPA-COF NSs. Reproduced with permission from ref. 107, Copyright the American Chemical Society (2017). under solvothermal conditions. ${ }^{107}$ The ultrathin NSs were well exploited for the highly selective and sensitive 'switch-on' sensing of DMA molecules, which mechanistically takes place exactly like the previous example in Fig. 67. Initially, two types of hairpin-like DNA probes, denoted as $\mathrm{H} 1$ and $\mathrm{H} 2$, were fabricated, one of which was FL tagged, and then allowed to adsorb on the TPA-COF nanosheets via $\pi-\pi$ stacking (Fig. 68). This led to fluorescence quenching, which was again revived by the hybridization chain reaction (HCR) between the two probes on the surface of the NSs. A long double-stranded DNA (dsDNA) was formed and detached from the NSs due to the weaker $\mathrm{p}-\mathrm{p}$ interactions. Furthermore, the fluorescence intensity was enhanced by increasing the concentration of the targeted DNA up to $1 \mathrm{nM}$ and a good linear relation was achieved. The LoD value for DNA in this case was found to be very low, i.e., $20 \mathrm{pM}$.

Myocardial infarction is a life-threatening cardiovascular disease, which can be prevented by the early detection of the biomarker cardiac troponin I (cTnI). In this direction, Zhang et al. reported an electrochemical immunosensor decorated with gold nanoparticles, $\mathrm{TiO}_{2}$-PPy $\left(\mathrm{TiO}_{2}\right.$-PPy-Au). ${ }^{258}$ This material was efficiently employed to detect cTnI quantitatively through the signal amplification strategy (Fig. 69). Initially, $\mathrm{TiO}_{2}-\mathrm{PPy}-\mathrm{Au}$ was decorated with primary antibodies $\left(\mathrm{AB}_{1}\right)$ on a GCE electrode. Conversely, the $\mathrm{Au}$ NP-decorated COF was subjected to secondary antibodies $\left(\mathrm{AB}_{2}\right)$ and toluidine blue (TB) aromatic dye via its amino-groups. The two units then behave as a sandwich-type electrochemical sensor for cTnI analyte. The proposed technique displayed a linear range from $0.5 \mathrm{pg} \mathrm{mL} \mathrm{m}^{-1}$ to $10.0 \mathrm{ng} \mathrm{mL}^{-1}$ together with a low detection limit of $0.17 \mathrm{pg} \mathrm{mL}^{-1}$.

In 2018, Wang et al. synthesized a porphyrin-based Fe-COF, which was utilized as a peroxidise mimic. ${ }^{259}$ The COF undergoes

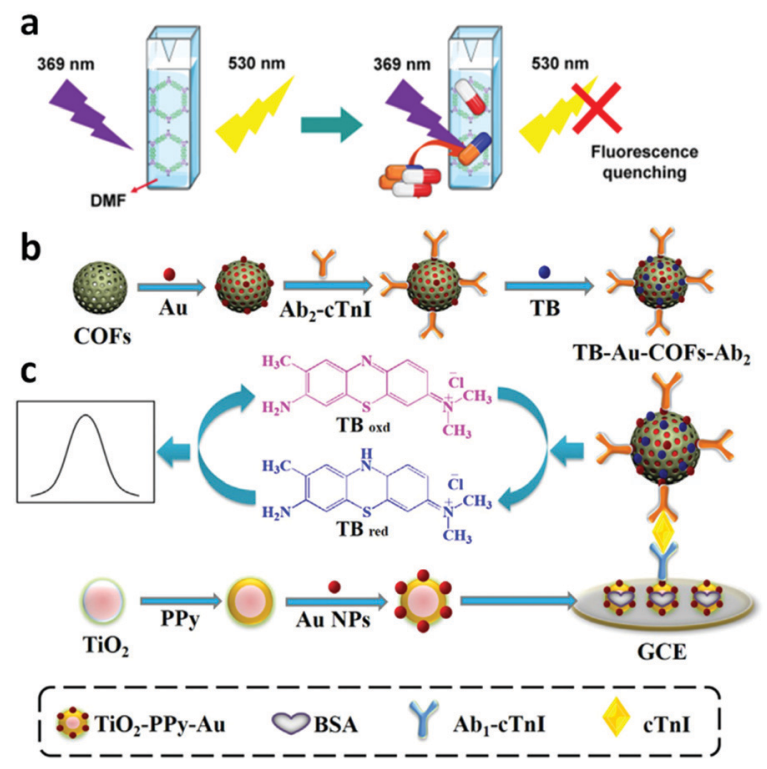

Fig. 69 (a) Process for the detection of antibiotics with COF-1. (b) Procedure for the preparation of the TB-Au-COFs-Ab2 labels. (c) Schematic illustration of the sandwich-type electrochemical immunosensor. Reproduced with permission from: (a) ref. 262. Copyright the American Chemical Society (2020) and (b) ref. 258. Copyright Elsevier (2018). 
colorimetric sensing of $\mathrm{H}_{2} \mathrm{O}_{2}$ and glucose molecules up to very low detection limit of 1.1 and $1.0 \mu \mathrm{M}$, respectively. The mechanism of the biosensing experiment involved the oxidation of the chromogenic substrate TMB in the presence of $\mathrm{H}_{2} \mathrm{O}_{2}$, where the peroxidise-mimetic Fe-porphyrin-COF captures $\mathrm{OH}$ radicals upon its decomposition. This was supported by observing the color change from colorless (for TMB) to blue (for oxTMB). After this, the framework was utilized for the detection of glucose. By combining the COF with glucose oxidase (GOx), the glucose molecules were oxidized to gluconic acid with high sensitivity and selectivity.

Recently, COFs have been utilized to detect $\mathrm{H}_{2} \mathrm{~S}$ gas in living cells, tumor tissues, etc. to diagnose neurodegenerative diseases. The COF TpASH was formed via the Schiff-base condensation of 1,3,5-triformylphloroglucinol (Tp) and the PTSA salt of 4- aminosalicylhydrazide (ASH), which was then tagged with a fluorescent probe via post-synthetic modification. ${ }^{111}$ The phenolic groups in TpASH were first alkylated by glycidol (Glc) on which a 4-amino1,8-naphthalimide derivative (NPHS) was anchored. This functionalized TpASH-NPHS framework was then exfoliated chemically to yield active nanosheets for the detection of $\mathrm{H}_{2} \mathrm{~S}^{260}$ In the presence of $\mathrm{H}_{2} \mathrm{~S}$, the azide units present on the NPHS-modified nanoprobe were reduced to amino group, causing an enhancement in the fluorescent emission with a detection limit of $0.11 \mu \mathrm{M}$. In addition, the TpASH-NPHS fluorescent nanoprobe was employed to detect and image the $\mathrm{H}_{2} \mathrm{~S}$ in deep tumor tissues, HeLa and HepG2, and in a cirrhotic liver.

Furthermore, chiral COFs (CCOFs) have been proven to be promising candidates for the enantioselective recognition of chiral molecules due to the presence of chiral-functionalized crown ether units in their framework. ${ }^{261}$ Crown ethers contain free electron pairs, and thus have the ability to form complexes with ammonium ions, binding them through $\mathrm{H}$-bonds. The formation of an adduct between the immobilized crown ether and protonated amines increases the interlayer spacing among the CCOF layers by weakening the $\pi-\pi$ interactions among them. This causes a change in fluorescent intensity.

In terms of food safety, the detection of antibiotics is necessary and provides insight in various food detection fields. Recently, attempts have been made in this direction. The green synthesis of a polyimide COF, named COF-1, was reported, showing very high luminescence at $530 \mathrm{~nm}\left(\lambda_{\mathrm{ex}}=369 \mathrm{~nm}\right)$ in $\mathrm{DMF}$, which is attributed to its perylene core and triazine monomeric units. Significant FL quenching was observed in the presence of the antibiotics ofloxacin and tetracycline, with a detection limit of 0.002 and $0.0065 \mathrm{mM}$, respectively, as shown in Fig. 69a. ${ }^{262}$

It is well known that antibiotics consist of various aromatic units, particularly, benzene ring units. Examples of these antibiotics are ampicillin (AMP) and enrofloxacin (ENR), which were effectively sensed by the novel pyrene-core Py-M-COF. ${ }^{263}$ This COF was constructed using melamine and TFPPy units, possessing a nanosheet-like structure, which is rich in functional groups such as amino, keto, imino and olefin groups. This enabled the framework to act as a good charge carrying probe. Moreover, the very high FL intensity of this framework is attributed to the presence of Py cores and extended $\pi$-conjugation. The COF behaves as an electrochemical aptasensor as it was first anchored with aptamers via $\pi-\pi$ stacking and electrostatic interactions. The ENR aptamers sense ENR molecules, and the AMP aptamers detect ANP molecules. There was an observable change in the confirmation and flexibility of the aptamer during the detection of AMP, whereas the ENR aptamer folded upon binding ENR. Extremely low detection limits for both antibiotics were calculated, i.e., 6.07 and $0.04 \mathrm{fg} \mathrm{mL}^{-1}(\mathrm{~S} / \mathrm{N}=3)$ for $\mathrm{ENR}$ and AMP, respectively.

6.2.7 Thermosensors. One of the essential but least explored field is the utility of COFs as thermosensors. Considering that temperature is a crucial parameter for many scientific domains, its detection by easily accessible materials is of great demand. Accordingly, Ln-grafted imine-linked COFs have been explored as temperature sensory materials. The incorporation of $\mathrm{Ln}^{3+}$ ions in the binding sites of COFs helps in their sensitization, provide well-separated sharp luminescent peaks, increased luminescence decay and high quantum yield values.

Voort and co-workers attempted the synthesis of the first TpBpy-COF grafted with various lanthanides $\left(\mathrm{Ln}^{3+}=\mathrm{Eu}^{3+}, \mathrm{Tb}^{3+}\right.$, $\mathrm{Eu}^{3+}-\mathrm{Tb}^{3+}$, and $\mathrm{Dy}^{3+}$ ) to serve as a heat detecting material in different temperature ranges. ${ }^{264}$ Initially, the TpBpy COF was synthesized solvothermally from triformylfluoroglucinol (Tp) and 2,2'-bipyridine-5, $5^{\prime}$-diamine (Bpy), which was then implanted with different $\mathrm{Ln}^{3+}$ ions using $\mathrm{Ln}(\mathrm{acac})_{3}\left(\mathrm{H}_{2} \mathrm{O}\right)_{2}$ and $\mathrm{LnCl}_{3}$. Among them, Eu, the Eu/Tb- and Dy-grafted systems were successfully employed as ratiometric thermometers in the temperature range of $10-360 \mathrm{~K}$ for the former two and 280$440 \mathrm{~K}$ for the latter (Fig. 70). In the $\mathrm{Eu}^{3+}$-sensitized TpBpy-COF (labelled 1), the sharp FL peak corresponding to $610 \mathrm{~nm}\left(\lambda_{\max }\right)$ with respect to the ${ }^{5} \mathrm{D}_{0} \rightarrow{ }^{7} \mathrm{~F}_{2}$ transition gradually decreased with an increase in temperature, whereas no significant change was found for the $\mathrm{Tb}^{3+}$-grafted COF (labelled 2), corresponding to the ${ }^{5} \mathrm{D}_{4} \rightarrow{ }^{7} \mathrm{~F}_{5}$ transition at the emission maximum of $548 \mathrm{~nm}$. This is ascribed by the absence of $\mathrm{Tb}^{3+}$-to-ligand back energy transfer due to the fact that the ${ }^{5} \mathrm{D}_{4}$-acceptor band lies above the donor triplet level of the TpBpy ligand, which is present for the former case. In the mixed ion-grafted COF (labelled 5) with an $\mathrm{Eu}^{3+}: \mathrm{Tb}^{3+}$ ratio of $80: 20$, a similar trend was observed given that the $\mathrm{Tb}^{3+}$-to- $\mathrm{Eu}^{3+}$ energy transfer is not possible due to the long distance between the ions. Therefore, $\mathrm{Tb}^{3+}$ did not show any quenching effect with an increase in temperature. A visible color change from orange-red to green for 5 was observed when the temperature was increased from $60 \mathrm{~K}$ to $360 \mathrm{~K}$. The results are highly convincing, with a thermal sensitivity of $1.403 \% \mathrm{~K}^{-1}(160 \mathrm{~K})$ and error bar of $\sim 1 \mathrm{~K}$ above 110 K. Moreover, the $\mathrm{Dy}^{3+}$ hybrid TpBpy COF also showed strong luminescence (482 nm, ${ }^{4} \mathrm{~F}_{9 / 2} \rightarrow{ }^{6} \mathrm{H}_{15 / 2}$ transition), which was gradually quenched with an increase in temperature from 280-440 K.

In 2020, another PP-TzDa COF was synthesized, which senses thermal changes in its environment without using $\mathrm{Ln}^{3+}$ ions. ${ }^{265}$ The PP-TzDa COF was obtained by mixing tri(4-aminophenyl)triazine and 2,5-dihydroxyterephthaldehyde as monomers, followed by post-synthetic modification with the chloro-derivative 
(a)

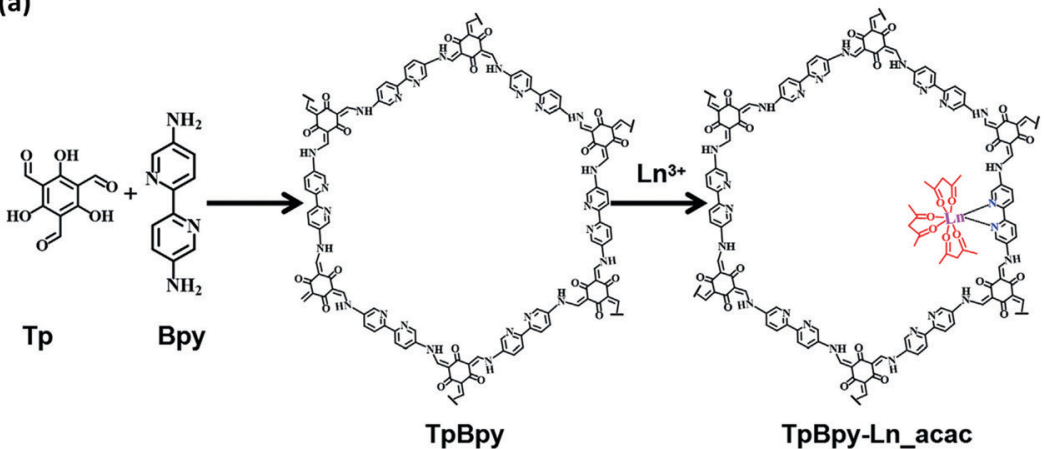

(b)

(c)
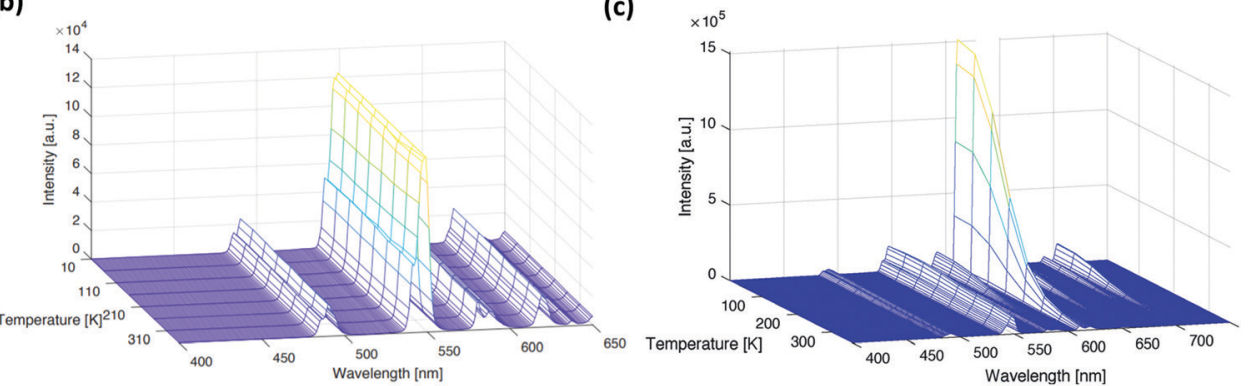

(d)

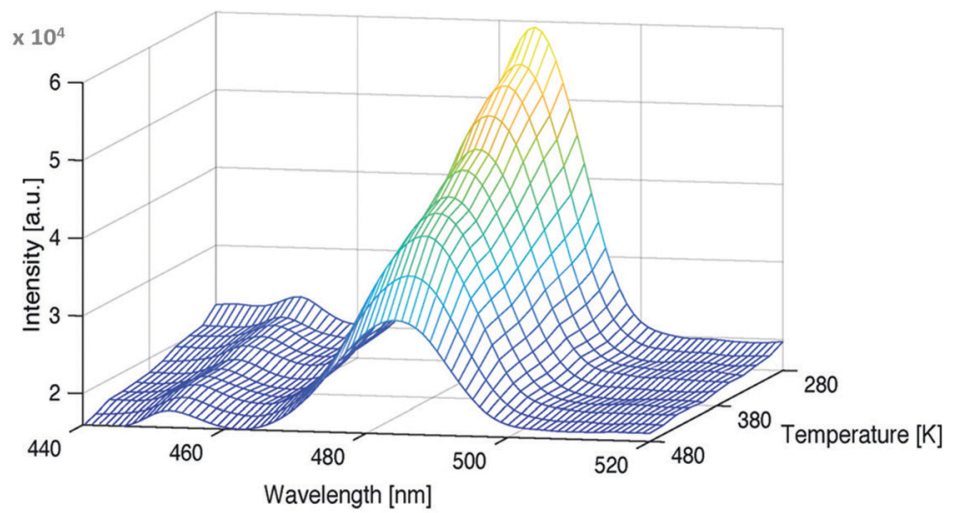

Fig. 70 (a) Schematic representation of TpBpy and TpBpy-Ln_acac COF materials. Emission map of (b) Tb ${ }^{3+}$-grafted and (c) Eu ${ }^{3+}:$ Tb $^{3+}(80: 20)-$ grafted TpBy COF recorded at 10-360 K and (d) Dy ${ }^{3+}$-grafted TpBy COF at 280-480 K. Reproduced with permission from ref. 264, Copyright John Wiley and Sons (2020).

of 2-phenylpropionic acid. The highly emissive COF exhibits luminescence due to (a) extended $\pi$-conjugation and (b) ESIPT causing dual-emission (530 and $597 \mathrm{~nm}$ ) due to the presence of $-\mathrm{OH}$ groups, which further react during PSM. The emission maximum at $597 \mathrm{~nm}$ is sharply quenched in the temperature range of $20-100{ }^{\circ} \mathrm{C}$ in DMF with a $\delta T$ higher than $0.1{ }^{\circ} \mathrm{C}$ and maximum temperature sensitivity of $0.95 \%$ per ${ }^{\circ} \mathrm{C}$ (Fig. 71 ).

According to the above-mentioned experiments, $\mathrm{Ln}^{3+}$-COF hybrids have been proven to be a new and emerging class of porous materials that can be exploited as luminescent thermometers. This year, another $4 \mathrm{f}$-3d-grafted COF was reported, in which the TTA-DFP COF was grafted with $\mathrm{Eu}^{3+}, \mathrm{Tb}^{3+}, \mathrm{Cu}^{2+}$ and $\mathrm{Ni}^{2+}$ ions. ${ }^{266}$ Subsequently, this COF was subjected to Eu(tfac $)_{3}$. $2 \mathrm{H}_{2} \mathrm{O}$ or $\mathrm{Tb}(\mathrm{tfac})_{3} \cdot 2 \mathrm{H}_{2} \mathrm{O}$ and $\mathrm{Cu}(\mathrm{OAc})_{2}$ one at a time, forming the Eu,Tb@TTA-DFPCOF@Cu material. It was further used for dual-ion thermometry application in the temperature range of $0-120{ }^{\circ} \mathrm{C}$ and $20-90{ }^{\circ} \mathrm{C}$ in toluene medium before and after co-grafting with $\mathrm{Cu}^{2+}$ ions, respectively. Interestingly, it was found that the Eu,Tb@TTA-DFP COF, before $\mathrm{Cu}^{2+}$-grafting, undergoes emission quenching with thermal sensitivity of $4.1 \%{ }^{\circ} \mathrm{C}$ at $42{ }^{\circ} \mathrm{C}$. Conversely, with the Cu-grafted Eu,Tb@TTA-DFP COF, there was an increase in emission intensity and good thermal uncertainty of $\delta T<0.37{ }^{\circ} \mathrm{C}$ in the range of $20-90{ }^{\circ} \mathrm{C}$ was observed. Being embedded with $\mathrm{Cu}^{2+} / \mathrm{Ln}^{3+}$ ions, this material was synchronously used as a thermometer and catalyst for the Glaser reaction.

\section{Concluding remarks and future perspectives}

In this review, we described the growth of COFs, starting from 2005 to date, to illustrate how this field has grown tremendously and attracted the attention of scientists at the global level. Recapitulating the main points, we briefly explained how the 

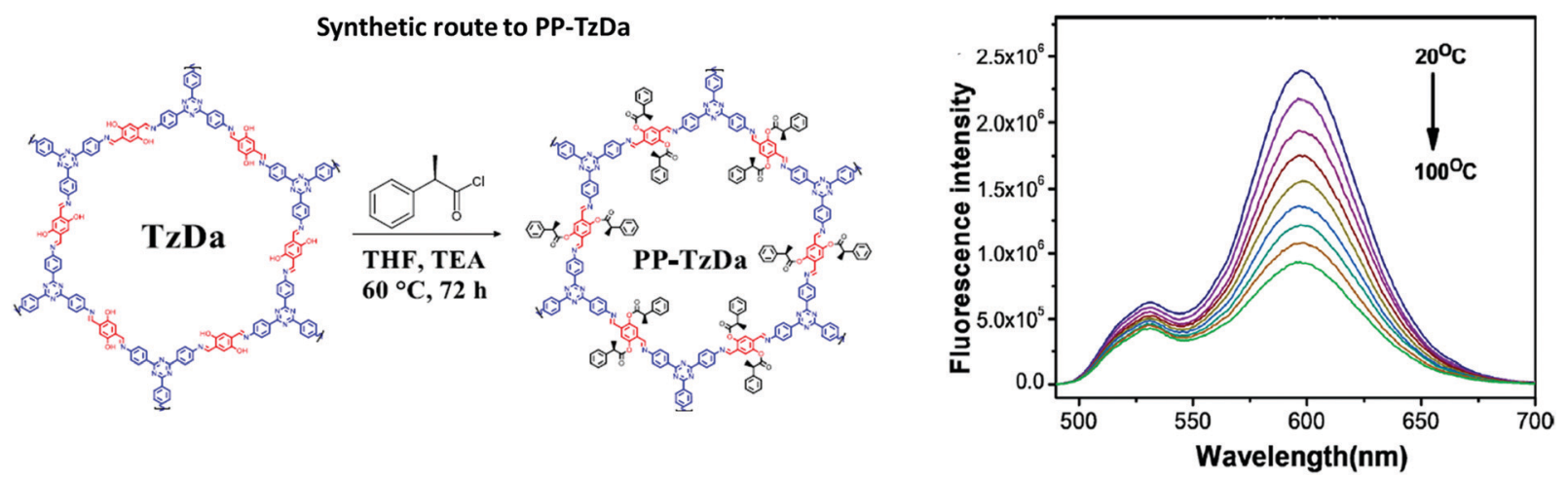

Fig. 71 (left) Synthetic route to PP-TzDa. (right) Fluorescence emission spectra of PP-TzDa in DMF ( $\lambda_{\text {ex }}=460 \mathrm{~nm} \mathrm{~nm}$ ) at $20-100{ }^{\circ} \mathrm{C}$. Reproduced with permission from ref. 265, Copyright Elsevier (2020).

choice of monomer, oriented at a particular angle with an appropriate geometry, tailors various networks with a number of topologies. Consequently, this affects various properties of the framework such as formation of highly ordered structures, its stability towards heat, chemical exposure and moisture, surface areas and pore size. The tremendous efforts in establishing various linkages to build the featured COF were explained in this review. The classification of COFs in 2D and 3D nets was thoroughly explained in the different sections. Additionally, the effect of stacking forces on the construction of 2D COFs and the property of interpenetration in 3D COFs were elaborated with various examples. We also described the various synthetic methodologies such as room temperature, hydro-/solvothermal, microwave, ionothermal, mechanochemical, interfacial and exfoliation methods adopted for these materials. Various analytical methods including FTIR, SS NMR, PXRD, SEM/TEM, XPS, BET and TGA have been efficiently exploited to characterize and monitor the formation of the framework. The structure modeling of most COFs is done with the help of PXRD data, which helps in predicting and establishing the application of these materials. Incorporating $\mathrm{N}$-rich linkages has promoted these frameworks to act as materials for the sensing of explosives, volatile organic compounds, metal ions, toxic anions, bioanalytes, temperature, $\mathrm{pH}$ and many more with high sensitivity and selectivity. The detection of these analytes follows several mechanisms, such as ESIPT, AIE/ACQ, PET, ICT and FRET, which were discussed in detail.

Nevertheless, there is a lot to be explored for COFs. Specifically, the three major areas further described below can provide tremendous scope for future research.

\section{(a) Framework construction}

There are many 2D and 3D frameworks reported thus far from the simplest and commonly found networks (hcb and dia) to the rarely obtained topologies (tth and bcu). Consideirng that the majority of COFs in the literature chose the $\left[C_{3}+C_{2}\right]$ or $\left[C_{3}+\right.$ $\left.C_{3}\right]$ combined hcb net and $\left[C_{4}+C_{2}\right]$ or $\left[C_{4}+C_{4}\right]$ combined dia net, it is necessary to explore high connectivity for both $2 \mathrm{D}$ and 3D COFs, respectively. As a new theme, exploring discrete molecular cages as building units can provide an interesting perspective to inflate the growth of porous networks.

\section{(b) New strategies for synthesis}

Since the solvothermal approach for the formation of first COF was carried out in 2005, this synthetic method has been largely exploited to tailor the desired framework. However, method uses harsh temperature and vacuum conditions together with the choice of non-green solvents to direct the COF reaction at the laboratory scale. Furthermore, strategic planning and vision are required to understand the formation and growth of suitable single crystals. Therefore, the following aspects need special attention:

(i) There is a dire need for the large-scale synthesis of robust frameworks using greener solvents. It must be noted that the high-scale production requires ambient reaction conditions.

(ii) The mechanistic work to establish a road map has been well done for 2D COFs forming boroxine and imine linkages with a few examples explaining the supramolecular interactions between the sheets. However, a more optimized and general method is needed for their synthesis. Henceforth, similar studies should be carried out for other widely explored linkages for a better understanding of the formation of COFs.

(iii) There are only a few single crystal structures known for 3D COF materials to date. Getting insight into the structure and establishing a structure-property relationship will open many other applications for these materials. Therefore, more efforts are needed to grow single crystals of these materials.

(iv) Finally, the post-synthetic modification of COFs is still a challenge with only a few examples known. This area can be explored further to increase the performance of COFs.

\section{(c) Sensing aspect}

Compared to other porous and highly stable materials for sensing applications, COFs are less explored for a number of reasons, as stated below. Therefore, finding proper solutions to these issues will definitely bring extraordinary results for showing the usefulness of one of the best porous materials to date.

(i) It has been observed that $\mathrm{N}$-rich $\mathrm{COF}$ nanosheets are more efficient towards sensing applications in comparison to bulk powders. However, very few CONs have been reported, which use limited synthetic methods. This area has a lot of 
scope in the formation of well-dispersed nanosheets for several real-time sensing applications.

(ii) Most COFs/CONs are studied for their chemical and thermal stability with a few examples of moisture stability. However, to utilize these materials for real-life applications, their stability towards moisture is of utmost importance. Thus, the design strategies have to be improved to generate materials with this property. For sensing applications, most of the experiments are done using organic solvents. However, for real-life applications, the functioning of sensors in water must be considered.

(iv) The regeneration and reusability of a sensor are always desirable. Thus, to maximize the efficiency of COFs as sensory materials, their regeneration and reusability should also be investigated.

(v) The utilization of COFs for temperature, $\mathrm{pH}$, anion and biomolecule sensing is underexplored. Furthermore, biocompatibility of COFs still needs to be determined for their use in the sensing of bioanalytes.

\section{Abbreviations}

\begin{tabular}{|c|c|}
\hline $\mathrm{ABN}$ & 4-Aminobenzonitrile \\
\hline $\mathrm{ACN}$ & Acetonitrile \\
\hline APPO & 3-Anilino-1-phenyl-2-propen-1-one \\
\hline BATH & 2,5-Bis(allyloxy)terephthalohydrazide \\
\hline BDA & Benzene-1,4-diamine \\
\hline $\mathrm{BDB}$ & $\begin{array}{l}\text { 1,1'-Bis }(2,4 \text {-dinitrophenyl)-[4,4'-bipyridine }] \text { - } \\
1,1^{\prime} \text {-diium dichloride }\end{array}$ \\
\hline BDBA & 1,4-Benzenediboronic acid \\
\hline BET & Brunauer-Emmett-Teller \\
\hline BFBZ & 4,7-Bis(4-formylbenzyl)-1H-benzimidazole \\
\hline BPDA & 4,4'-Biphenyldicarbaldehyde \\
\hline BPDAN & 2,2'-(Biphenyl-4,4'-diyl)diacetonitrile \\
\hline BTCA & 1,3,5-Benzenetricarboxaldehyde \\
\hline BTCH & Benzene-1,3,5-tricarbohydrazide \\
\hline ВTTPA & $5,5^{\prime}, 5^{\prime \prime}$-(Benzene-1,3,5-triyl)tripicolinaldehyde \\
\hline $\mathrm{CC}$ & Cyanuric chloride \\
\hline CHDA & trans-1,4-Cyclohexyldiamine \\
\hline $\mathrm{DAB}$ & 3,3'-Diaminobenzidine \\
\hline DABD & 2,5-Diamino-1,4-benzenediol dihydrochloride \\
\hline DAHQ-2HCl & 2,5-Diaminohydroquinone dihydrochloride \\
\hline DBU & 1,8-Diazabicyclo[5.4.0]undec-7-ene \\
\hline DCTMP & 3,5-Dicyano-2,4,6-trimethylpyridine \\
\hline DCTSP & 3,5-Dicyano-2,4,6-tristyrylpyridine \\
\hline DETHz & 2,5-Diethoxyterephthalohydrazide \\
\hline DFPBA & 3,5-Diformylphenylboronic acid \\
\hline Dha & 2,5-Dihydroxyterephthalaldehyde \\
\hline DHTA & 2,4-Dihydroxybenzene-1,3,5-tricarbaldehyde \\
\hline 2,3-DHTH & 2,3-Dihydroxyterephthalohydrazide \\
\hline 2,5-DHTH & 2,5-Dihydroxyterephthalohydrazide \\
\hline DMA & Dimethyl acetamide \\
\hline Dma & 2,5-Dimethoxyterephthalaldehyde \\
\hline DMB & 2,5-Dimethyl-1,4-benzenediamine \\
\hline DMOB & 3,3'-Dimethoxybenzidine \\
\hline
\end{tabular}

DMPO

DMTH

DMTHA

DNB

DNP

DPBF

DPBP

DPPO

DPT

DPTH

$\mathrm{Et}_{3} \mathrm{~N}$

FPBA

FTIR

HHTP

HOPG

HTA

iPrTAPB

MOP

MTAB

NMP

NP

NT

$\mathrm{ODH}$

OHPM

PATCnC

PDA

PDAN

PMDA

PMMA

PT

PTFE

PXRD

SEM

TAA

TAB

TABPB

TA

TAP

TAPA

TAPB

TAPM

TAPT

TATP

TATTA

TCB

TCTAB

TDOEB

TEM

TFB
5,5-Dimethyl-1-pyrroline $N$-oxide

2,5-Dimethoxyterephthalohydrazide

9,10-Dimethyl-2,3,6,7-tetrahydroxyanthracene

3,3'-Dinitrobenzidine

2,4-Dinitrophenol

1,3-Diphenylisobenzofuran

Di(pyridin-2-yl) [1,1'-biphenyl]-4,4'-dicarboxylate

3-(Dimethylamino)-1-phenyl-2-propen-1-one

Di(pyridin-2-yl) terephthalate

2,5-Bis(prop-2-yn-1-yloxy)terephthalohydrazide

Triethylamine

4-Formylphenylboronic acid

Fourier Transform Infrared Spectroscopy

2,3,6,7,10,11-Hexadydroxytriphenylene

Highly oriented pyrolytic graphite

2-Hydroxybenzene-1,3,5-tricarbaldehyde

1,3,5-Tris ( $4^{\prime}$-amino- $3^{\prime}, 5^{\prime}$ -

isopropylphenyl)benzene

Microporous organic polymers

$4,4^{\prime}, 4^{\prime \prime}, 4^{\prime \prime \prime}$-Methanetetrayltetrabenzoic acid

$\mathrm{N}$-Methyl-2-pyrrolidinone

2-Nitrophenol

2-Nitrotoluene

Oxalyldihydrazide

$2,3,9,10,16,17,23,24-$

Octahydroxyphthalocyaninato metal

Polyamide $\left(4,4^{\prime}, 4^{\prime \prime}, 4^{\prime \prime \prime}\right.$ -

tetra(carboxyphenyl)methane-net-trans-1,4-

cyclohexanediamine)

Phenylenediamine

2,2'-(1,4-Phenylene)diacetonitrile

Pyromelliticdianhydride

Polymethylmethacrylate

tert-Butyl pyrene-tetraone

Polytetrafluoroethylene

Powder X-ray diffractometry

Scanning electron microscopy

1,3,5,7-Tetraaminoadamantane

1,2,4,5-Tetraminobenzene

1,3,5-Tris[4-amino(1,1-biphenyl-4-yl)]benzene

Terephthaldehyde

5,10,15,20-Tetrakis(4-aminophenyl)porphyrin

Tris(4-aminophenyl)amine

1,3,5-Tris ( $4^{\prime}$-aminophenyl)benzene

Tetra(4-aminophenyl)methane

1,3,5-Tris(4-aminophenyl)triazine

Triacetonetriperoxide

$4,4^{\prime}, 4^{\prime \prime}$-(1,3,5-Triazine-2,4,6-

triyl)tribenzaldehyde

1,3,5-Tricyanobenzene

1,3,5-Tricarboxylicacid-tris(4-amino-phenyl-

amide)benzene

1,3,5-Tris(3-dimethylamino-1-oxoprop-2-en-

yl)benzene

Transmission electron microscopy

1,3,5-Triformylbenzene 


$\begin{array}{ll}\text { TFFPB } & \text { 1,3,5-Tris(3-fluoro-4-formyl phenyl)benzene } \\ \text { TFM } & \text { Tetrakis(4-formylphenyl)methane } \\ \text { TFMS } & \text { Trifluoromethanesulfonic acid } \\ \text { TFP } & \text { 1,3,5-Triformylphluroglucinol } \\ \text { Tfpa } & \text { Tris(4-formylphenyl)amine } \\ \text { TFPB } & \text { 1,3,5-Tris(4'-formylphenyl)benzene } \\ \text { TFPC } & \text { 2,3,5,6-Tetrafluoro-4-pyridinecarbonitrile } \\ \text { TFPC } & \text { Tri(4-formylphenoxy)cyanurate } \\ \text { TFPN } & \text { Tetrafluorophthalonitrile } \\ \text { TFPPy } & \text { 1,3,6,8-Tetrakis(4-formylphenyl)pyrene } \\ \text { TFPT } & \text { 1,3,5-Tris(4-formyl-phenyl)triazine } \\ \text { THA } & \text { Terephthalohydrazide } \\ \text { THAT } & \text { 4,6-Trihydroxybenzene-1,3,5-tricarbaldehyde } \\ \text { THPB } & \text { 1,3,5-Tris(4-hydroxyphenyl)benzene } \\ \text { THPE } & \text { Tetrakis(4-hydroxyphenyl)ethylene } \\ \text { Thz } & \text { Terephthalicdihydrazide } \\ \text { TICPB } & \text { 1,3,5-Tris(4-isocyanophenyl)benzene } \\ \text { TGA } & \text { Thermogravimetric Analysis } \\ \text { TMA } & \text { Trimesic acid } \\ \text { TMEDA } & \text { Tetramethylethylenediamine } \\ \text { TMOS } & \text { Tetramethylorthosilicate } \\ \text { TMSCN } & \text { Trimethylsilyl cyanide } \\ \text { TMTA } & \text { 2,4,6-Trimethyl-1,3,5-triazine } \\ \text { TNP } & \text { 2,4,6-Trinitrophenol } \\ \text { Tp } & \text { 1,3,5-Triformylphloroglucinol } \\ \text { TPDAN } & \text { 2,2'-([1,1' }: 4^{\prime}, 1^{\prime \prime} \text {-Terphenyl]-4,4'- } \\ \text { TPHA } & \text { diyl)diacetonitrile } \\ \text { TPT-3CHO } & \text { Triphenylene hexamine } \\ \text { TST } & \text { 2,4,6-Tris(4-formylphenyl)triazine } \\ \text { XPS } & \text { 2,4,6-Tri((E)-styryl)-1,3,5-triazine } \\ & \end{array}$

\section{Author contributions}

All authors contributed to the organization and writing of this manuscript. Its final version was approved by all authors.

\section{Note after first publication}

This article replaces the version published on $23^{\text {rd }}$ November 2021, which contained errors in Table 3.

\section{Conflicts of interest}

There are no conflicts to declare.

\section{Acknowledgements}

H. B. is grateful to the UGC of India for a research fellowship. Sakshi is grateful to MHRD, India for a research fellowship.

\section{Notes and references}

1 W. B. Jensen, J. Chem. Educ., 2008, 85, 624.
2 H. Staudinger and J. Fritschi, Helv. Chim. Acta, 1922, 5, 785-806.

3 J.-F. Lutz, M. Ouchi, D. R. Liu and M. Sawamoto, Science, 2013, 341, 1238149.

4 A. P. Cote, A. I. Benin, N. W. Ockwig, M. O'Keeffe, A. J. Matzger and O. M. Yaghi, Science, 2005, 310, 1166-1170.

5 B. J. Smith and W. R. Dichtel, J. Am. Chem. Soc., 2014, 136, 8783-8789.

6 T. Ma, E. A. Kapustin, S. X. Yin, L. Liang, Z. Zhou, J. Niu, L.-H. Li, Y. Wang, J. Su, J. Li, X. Wang, W. D. Wang, W. Wang, J. Sun and O. M. Yaghi, Science, 2018, 361, 48-52.

7 B. C. Patra, S. Khilari, R. N. Manna, S. Mondal, D. Pradhan, A. Pradhan and A. Bhaumik, ACS Catal., 2017, 7, 6120-6127.

8 J. Guo and D. Jiang, ACS Cent. Sci., 2020, 6, 869-879.

9 K. Zhang, K. O. Kirlikovali, R. S. Varma, Z. Jin, H. W. Jang, O. K. Farha and M. Shokouhimehr, ACS Appl. Mater. Interfaces, 2020, 12, 27821-27852.

10 W. Wang, V. S. Kale, Z. Cao, S. Kandambeth, W. Zhang, J. Ming, P. T. Parvatkar, E. Abou-Hamad, O. Shekhah, L. Cavallo, M. Eddaoudi and H. N. Alshareef, ACS Energy Lett., 2020, 5, 2256-2264.

11 Y. Song, Q. Sun, B. Aguila and S. Ma, Adv. Sci., 2019, 6, 1801410.

12 X. Liu, D. Huang, C. Lai, G. Zeng, L. Qin, H. Wang, H. Yi, B. Li, S. Liu, M. Zhang, R. Deng, Y. Fu, L. Li, W. Xue and S. Chen, Chem. Soc. Rev., 2019, 48, 5266-5302.

13 T. Skorjanc, D. Shetty and M. Valant, ACS Sens., 2021, 6, 1461-1481.

14 X. Chen, K. Geng, R. Liu, K. T. Tan, Y. Gong, Z. Li, S. Tao, Q. Jiang and D. Jiang, Angew. Chem., Int. Ed., 2020, 59, 5050-5091.

15 E. L. Spitler and W. R. Dichtel, Nat. Chem., 2010, 2, 672-677.

16 L. Liang, Y. Qiu, W. D. Wang, J. Han, Y. Luo, W. Yu, G. Yin, Z. Wang, L. Zhang, J. Ni, J. Niu, J. Sun, T. Ma and W. Wang, Angew. Chem., Int. Ed., 2020, 59, 17991-17995.

17 S. Wan, J. Guo, J. Kim, H. Ihee and D. Jiang, Angew. Chem., Int. Ed., 2009, 48, 5439-5442.

18 E. L. Spitler, B. T. Koo, J. L. Novotney, J. W. Colson, F. J. Uribe-Romo, G. D. Gutierrez, P. Clancy and W. R. Dichtel, J. Am. Chem. Soc., 2011, 133, 19416-19421.

19 P. Katekomol, J. Roeser, M. Bojdys, J. Weber and A. Thomas, Chem. Mater., 2013, 25, 1542-1548.

20 P. Kuhn, M. Antonietti and A. Thomas, Angew. Chem., Int. Ed., 2008, 47, 3450-3453.

21 W. Huang, J. Byun, I. Rörich, C. Ramanan, P. W. M. Blom, H. Lu, D. Wang, L. Caire da Silva, R. Li, L. Wang, K. Landfester and K. A. I. Zhang, Angew. Chem., Int. Ed., 2018, 57, 8316-8320.

22 F. J. Uribe-Romo, C. J. Doonan, H. Furukawa, K. Oisaki and O. M. Yaghi, J. Am. Chem. Soc., 2011, 133, 11478-11481.

23 A. P. Côté, H. M. El-Kaderi, H. Furukawa, J. R. Hunt and O. M. Yaghi, J. Am. Chem. Soc., 2007, 129, 12914-12915.

24 P. Das and S. K. Mandal, J. Mater. Chem. A, 2018, 6, 16246-16256. 25 S.-Q. Xu, T.-G. Zhan, Q. Wen, Z.-F. Pang and X. Zhao, ACS Macro Lett., 2016, 5, 99-102.

26 S. Dalapati, M. Addicoat, S. Jin, T. Sakurai, J. Gao, H. Xu, S. Irle, S. Seki and D. Jiang, Nat. Commun., 2015, 6, 7786. 
27 L. Ascherl, T. Sick, J. T. Margraf, S. H. Lapidus, M. Calik, C. Hettstedt, K. Karaghiosoff, M. Döblinger, T. Clark, K. W. Chapman, F. Auras and T. Bein, Nat. Chem., 2016, 8, 310-316.

28 T.-Y. Zhou, S.-Q. Xu, Q. Wen, Z.-F. Pang and X. Zhao, J. Am. Chem. Soc., 2014, 136, 15885-15888.

29 S. Dalapati, E. Jin, M. Addicoat, T. Heine and D. Jiang, J. Am. Chem. Soc., 2016, 138, 5797-5800.

$30 \mathrm{Y}$. $\mathrm{Wu}, \mathrm{H} . \mathrm{Xu}, \mathrm{X}$. Chen, J. Gao and D. Jiang, Chem. Commun., 2015, 51, 10096-10098.

31 L. Wang, C. Zeng, H. Xu, P. Yin, D. Chen, J. Deng, M. Li, N. Zheng, C. Gu and Y. Ma, Chem. Sci., 2019, 10, 1023-1028.

32 A. Cuevas, I. Viera, M. H. Torre, E. Kremer, S. B. Etcheverry and E. J. Baran, Afinidad, 1999, 56, 263-265.

33 S.-Q. Xu, R.-R. Liang, T.-G. Zhan, Q.-Y. Qi and X. Zhao, Chem. Commun., 2017, 53, 2431-2434.

34 B. Zhang, H. Mao, R. Matheu, J. A. Reimer, S. A. Alshmimri, S. Alshihri and O. M. Yaghi, J. Am. Chem. Soc., 2019, 141, 11420-11424.

35 B. Nath, W.-H. Li, J.-H. Huang, G.-E. Wang, Z. Fu, M.-S. Yao and G. Xu, CrystEngComm, 2016, 18, 4259-4263.

36 C. Qian, Q.-Y. Qi, G.-F. Jiang, F.-Z. Cui, Y. Tian and X. Zhao, J. Am. Chem. Soc., 2017, 139, 6736-6743.

37 Y. Du, H. Yang, J. M. Whiteley, S. Wan, Y. Jin, S.-H. Lee and W. Zhang, Angew. Chem., Int. Ed., 2016, 55, 1737-1741.

38 S.-Y. Jiang, S.-X. Gan, X. Zhang, H. Li, Q.-Y. Qi, F.-Z. Cui, J. Lu and X. Zhao, J. Am. Chem. Soc., 2019, 141, 14981-14986.

39 T. Banerjee, F. Haase, S. Trenker, B. P. Biswal, G. Savasci, V. Duppel, I. Moudrakovski, C. Ochsenfeld and B. V. Lotsch, Nat. Commun., 2019, 10, 2689.

40 H. L. Nguyen, N. Hanikel, S. J. Lyle, C. Zhu, D. M. Proserpio and O. M. Yaghi, J. Am. Chem. Soc., 2020, 142, 2218-2221.

41 S.-L. Cai, Z.-H. He, X.-L. Li, K. Zhang, S.-R. Zheng, J. Fan, Y. Liu and W.-G. Zhang, Chem. Commun., 2019, 55, 13454-13457.

42 X. Guan, F. Chen, Q. Fang and S. Qiu, Chem. Soc. Rev., 2020, 49, 1357-1384.

43 C. Bonneau, O. Delgado-Friedrichs, M. O'Keeffe and O. M. Yaghi, Acta Crystallogr., Sect. A: Found. Crystallogr., 2004, 60, 517-520.

44 Q. Fang, J. Wang, S. Gu, R. B. Kaspar, Z. Zhuang, J. Zheng, H. Guo, S. Qiu and Y. Yan, J. Am. Chem. Soc., 2015, 137, 8352-8355.

45 H. M. El-Kaderi, J. R. Hunt, J. L. Mendoza-Cortes, A. P. Cote, R. E. Taylor, M. O'Keeffe and O. M. Yaghi, Science, 2007, 316, 268-272.

46 G. Lin, H. Ding, D. Yuan, B. Wang and C. Wang, J. Am. Chem. Soc., 2016, 138, 3302-3305.

47 Y. Xie, J. Li, C. Lin, B. Gui, C. Ji, D. Yuan, J. Sun and C. Wang, J. Am. Chem. Soc., 2021, 143, 7279-7284.

48 Y. Zhang, J. Duan, D. Ma, P. Li, S. Li, H. Li, J. Zhou, X. Ma, X. Feng and B. Wang, Angew. Chem., Int. Ed., 2017, 56, 16313-16317.

49 O. Yahiaoui, A. N. Fitch, F. Hoffmann, M. Fröba, A. Thomas and J. Roeser, J. Am. Chem. Soc., 2018, 140, 5330-5333.

50 X. Kang, X. Wu, X. Han, C. Yuan, Y. Liu and Y. Cui, Chem. Sci., 2020, 11, 1494-1502.
51 X. Kang, X. Han, C. Yuan, C. Cheng, Y. Liu and Y. Cui, J. Am. Chem. Soc., 2020, 142, 16346-16356.

52 H. L. Nguyen, C. Gropp, Y. Ma, C. Zhu and O. M. Yaghi, J. Am. Chem. Soc., 2020, 142, 20335-20339.

53 H. Li, J. Ding, X. Guan, F. Chen, C. Li, L. Zhu, M. Xue, D. Yuan, V. Valtchev, Y. Yan, S. Qiu and Q. Fang, J. Am. Chem. Soc., 2020, 142, 13334-13338.

54 Y. Wang, C. Wu, W. Sun, Q. Pan, W. Hao, H. Liu, J. Sun, Z. Li, J. Sun and Y. Zhao, Mater. Chem. Front., 2021, 5, 944-949.

55 Q. Zhu, X. Wang, R. Clowes, P. Cui, L. Chen, M. A. Little and A. I. Cooper, J. Am. Chem. Soc., 2020, 142, 16842-16848.

56 Z. Li, L. Sheng, H. Wang, X. Wang, M. Li, Y. Xu, H. Cui, H. Zhang, H. Liang, H. Xu and X. He, J. Am. Chem. Soc., 2021, 143, 92-96.

57 C. Gropp, T. Ma, N. Hanikel and O. M. Yaghi, Science, 2020, 370, eabd6406.

58 F. Haase and B. V. Lotsch, Chem. Soc. Rev., 2020, 49, 8469-8500.

59 L. M. Lanni, R. W. Tilford, M. Bharathy and J. J. Lavigne, J. Am. Chem. Soc., 2011, 133, 13975-13983.

60 K. T. Jackson, T. E. Reich and H. M. El-Kaderi, Chem. Commun., 2012, 48, 8823.

61 J. R. Hunt, C. J. Doonan, J. D. LeVangie, A. P. Côté and O. M. Yaghi, J. Am. Chem. Soc., 2008, 130, 11872-11873.

62 B. Zhang, M. Wei, H. Mao, X. Pei, S. A. Alshmimri, J. A. Reimer and O. M. Yaghi, J. Am. Chem. Soc., 2018, 140, 12715-12719.

63 C. Zhao, H. Lyu, Z. Ji, C. Zhu and O. M. Yaghi, J. Am. Chem. Soc., 2020, 142, 14450-14454.

64 Y. Zeng, R. Zou, Z. Luo, H. Zhang, X. Yao, X. Ma, R. Zou and Y. Zhao, J. Am. Chem. Soc., 2015, 137, 1020-1023.

65 H. Wang, C. Qian, J. Liu, Y. Zeng, D. Wang, W. Zhou, L. Gu, H. Wu, G. Liu and Y. Zhao, J. Am. Chem. Soc., 2020, 142, 4862-4871.

66 R.-R. Liang, R.-H. A, S.-Q. Xu, Q.-Y. Qi and X. Zhao, J. Am. Chem. Soc., 2020, 142, 70-74.

67 S. Kandambeth, A. Mallick, B. Lukose, M. V. Mane, T. Heine and R. Banerjee, J. Am. Chem. Soc., 2012, 134, 19524-19527.

68 P. Das and S. K. Mandal, Chem. Mater., 2019, 31, 1584-1596.

69 D. A. Pyles, J. W. Crowe, L. A. Baldwin and P. L. McGrier, ACS Macro Lett., 2016, 5, 1055-1058.

70 P. J. Waller, Y. S. AlFaraj, C. S. Diercks, N. N. Jarenwattananon and O. M. Yaghi, J. Am. Chem. Soc., 2018, 140, 9099-9103.

71 W. Liu, X. Luo, Y. Bao, Y. P. Liu, G.-H. Ning, I. Abdelwahab, L. Li, C. T. Nai, Z. G. Hu, D. Zhao, B. Liu, S. Y. Quek and K. P. Loh, Nat. Chem., 2017, 9, 563-570.

72 W. Liu, M. Ulaganathan, I. Abdelwahab, X. Luo, Z. Chen, S. J. Rong Tan, X. Wang, Y. Liu, D. Geng, Y. Bao, J. Chen and K. P. Loh, ACS Nano, 2018, 12, 852-860.

73 D. Zhou, X. Tan, H. Wu, L. Tian and M. Li, Angew. Chem., Int. Ed., 2019, 58, 1376-1381.

74 S. Wu, M. Li, H. Phan, D. Wang, T. S. Herng, J. Ding, Z. Lu and J. Wu, Angew. Chem., Int. Ed., 2018, 57, 8007-8011. 
75 D. L. Pastoetter, S. Xu, M. Borrelli, M. Addicoat, B. P. Biswal, S. Paasch, A. Dianat, H. Thomas, R. Berger, S. Reineke, E. Brunner, G. Cuniberti, M. Richter and X. Feng, Angew. Chem., Int. Ed., 2020, 59, 23620-23625.

$76 \mathrm{~J} . \mathrm{Hu}, \mathrm{S}$. K. Gupta, J. Ozdemir and M. H. Beyzavi, ACS Appl. Nano Mater., 2020, 3, 6239-6269.

77 L. Bourda, C. Krishnaraj, P. Van Der Voort and K. Van Hecke, Mater. Adv., 2021, 2, 2811-2845.

78 S. B. Alahakoon, S. D. Diwakara, C. M. Thompson and R. A. Smaldone, Chem. Soc. Rev., 2020, 49, 1344-1356.

79 A. Halder, S. Kandambeth, B. P. Biswal, G. Kaur, N. C. Roy, M. Addicoat, J. K. Salunke, S. Banerjee, K. Vanka, T. Heine, S. Verma and R. Banerjee, Angew. Chem., Int. Ed., 2016, 55, 7806-7810.

80 A. Halder, S. Karak, M. Addicoat, S. Bera, A. Chakraborty, S. H. Kunjattu, P. Pachfule, T. Heine and R. Banerjee, Angew. Chem., Int. Ed., 2018, 57, 5797-5802.

81 C. Zhao, C. S. Diercks, C. Zhu, N. Hanikel, X. Pei and O. M. Yaghi, J. Am. Chem. Soc., 2018, 140, 16438-16441.

82 F. J. Uribe-Romo, J. R. Hunt, H. Furukawa, C. Klöck, M. O’Keeffe and O. M. Yaghi, J. Am. Chem. Soc., 2009, 131, 4570-4571.

83 B. P. Biswal, S. Chandra, S. Kandambeth, B. Lukose, T. Heine and R. Banerjee, J. Am. Chem. Soc., 2013, 135, 5328-5331.

84 H. Wei, S. Chai, N. Hu, Z. Yang, L. Wei and L. Wang, Chem. Commun., 2015, 51, 12178-12181.

85 X. Guan, Y. Ma, H. Li, Y. Yusran, M. Xue, Q. Fang, Y. Yan, V. Valtchev and S. Qiu, J. Am. Chem. Soc., 2018, 140, 4494-4498.

86 D. Stewart, D. Antypov, M. S. Dyer, M. J. Pitcher, A. P. Katsoulidis, P. A. Chater, F. Blanc and M. J. Rosseinsky, Nat. Commun., 2017, 8, 1102.

87 Z. Li, X. Ding, Y. Feng, W. Feng and B.-H. Han, Macromolecules, 2019, 52, 1257-1265.

88 D. Beaudoin, T. Maris and J. D. Wuest, Nat. Chem., 2013, 5, 830-834.

89 Y.-B. Zhang, J. Su, H. Furukawa, Y. Yun, F. Gándara, A. Duong, X. Zou and O. M. Yaghi, J. Am. Chem. Soc., 2013, 135, 16336-16339.

90 B. Hou, S. Yang, K. Yang, X. Han, X. Tang, Y. Liu, J. Jiang and Y. Cui, Angew. Chem., 2021, 133, 6151-6158.

91 T. Ma, J. Li, J. Niu, L. Zhang, A. S. Etman, C. Lin, D. Shi, P. Chen, L.-H. Li, X. Du, J. Sun and W. Wang, J. Am. Chem. Soc., 2018, 140, 6763-6766.

92 C. Gao, J. Li, S. Yin, J. Sun and C. Wang, J. Am. Chem. Soc., 2020, 142, 3718-3723.

93 L. Zhu and Y.-B. Zhang, Molecules, 2017, 22, 1149.

94 G. Das, D. Balaji Shinde, S. Kandambeth, B. P. Biswal and R. Banerjee, Chem. Commun., 2014, 50, 12615-12618.

95 N. L. Campbell, R. Clowes, L. K. Ritchie and A. I. Cooper, Chem. Mater., 2009, 21, 204-206.

96 W. Ji, Y.-S. Guo, H.-M. Xie, X. Wang, X. Jiang and D.-S. Guo, J. Hazard. Mater., 2020, 397, 122793.

97 A. de la Peña Ruigómez, D. Rodríguez-San-Miguel, K. C. Stylianou, M. Cavallini, D. Gentili, F. Liscio, S. Milita, O. M. Roscioni, M. L. Ruiz-González, C. Carbonell, D. Maspoch, R. Mas-Ballesté, J. L. Segura and F. Zamora, Chem. - Eur. J., 2015, 21, 10666-10670.
98 D. Rodríguez-San-Miguel, A. Yazdi, V. Guillerm, J. PérezCarvajal, V. Puntes, D. Maspoch and F. Zamora, Chem. Eur. J., 2017, 23, 8623-8627.

99 T. Shiraki, G. Kim and N. Nakashima, Chem. Lett., 2015, 44, 1488-1490.

100 K. Dey, M. Pal, K. C. Rout, S. Kunjattu, H. A. Das, R. Mukherjee, U. K. Kharul and R. Banerjee, J. Am. Chem. Soc., 2017, 139, 13083-13091.

101 X.-H. Liu, C.-Z. Guan, S.-Y. Ding, W. Wang, H.-J. Yan, D. Wang and L.-J. Wan, J. Am. Chem. Soc., 2013, 135, 10470-10474.

102 J. I. Feldblyum, C. H. McCreery, S. C. Andrews, T. Kurosawa, E. J. G. Santos, V. Duong, L. Fang, A. L. Ayzner and Z. Bao, Chem. Commun., 2015, 51, 13894-13897.

103 A. Gogia, P. Das and S. K. Mandal, ACS Appl. Mater. Interfaces, 2020, 12, 46107-46118.

104 G. Li, K. Zhang and T. Tsuru, ACS Appl. Mater. Interfaces, 2017, 9, 8433-8436.

105 I. Berlanga, M. L. Ruiz-González, J. M. González-Calbet, J. L. G. Fierro, R. Mas-Ballesté and F. Zamora, Small, 2011, 7, 1207-1211.

106 D. N. Bunck and W. R. Dichtel, J. Am. Chem. Soc., 2013, 135, 14952-14955.

107 Y. Peng, Y. Huang, Y. Zhu, B. Chen, L. Wang, Z. Lai, Z. Zhang, M. Zhao, C. Tan, N. Yang, F. Shao, Y. Han and H. Zhang, J. Am. Chem. Soc., 2017, 139, 8698-8704.

108 S. Chandra, S. Kandambeth, B. P. Biswal, B. Lukose, S. M. Kunjir, M. Chaudhary, R. Babarao, T. Heine and R. Banerjee, J. Am. Chem. Soc., 2013, 135, 17853-17861.

109 S. Wang, Q. Wang, P. Shao, Y. Han, X. Gao, L. Ma, S. Yuan, X. Ma, J. Zhou, X. Feng and B. Wang, J. Am. Chem. Soc., 2017, 139, 4258-4261.

110 M. A. Khayum, S. Kandambeth, S. Mitra, S. B. Nair, A. Das, S. S. Nagane, R. Mukherjee and R. Banerjee, Angew. Chem., Int. Ed., 2016, 55, 15604-15608.

111 S. Mitra, H. S. Sasmal, T. Kundu, S. Kandambeth, K. Illath, D. Díaz Díaz and R. Banerjee, J. Am. Chem. Soc., 2017, 139, 4513-4520.

112 S. Haldar, K. Roy, S. Nandi, D. Chakraborty, D. Puthusseri, Y. Gawli, S. Ogale and R. Vaidhyanathan, Adv. Energy Mater., 2018, 8, 1702170.

113 S. Mitra, S. Kandambeth, B. P. Biswal, A. Khayum, M. C. K. Choudhury, M. Mehta, G. Kaur, S. Banerjee, A. Prabhune, S. Verma, S. Roy, U. K. Kharul and R. Banerjee, J. Am. Chem. Soc., 2016, 138, 2823-2828.

114 H. Singh, M. Devi, N. Jena, M. M. Iqbal, Y. Nailwal, A. De Sarkar and S. K. Pal, ACS Appl. Mater. Interfaces, 2020, 12, 13248-13255.

115 R. P. Bisbey, C. R. DeBlase, B. J. Smith and W. R. Dichtel, J. Am. Chem. Soc., 2016, 138, 11433-11436.

116 Y. Peng, W. K. Wong, Z. Hu, Y. Cheng, D. Yuan, S. A. Khan and D. Zhao, Chem. Mater., 2016, 28, 5095-5101.

117 J. W. Colson, A. R. Woll, A. Mukherjee, M. P. Levendorf, E. L. Spitler, V. B. Shields, M. G. Spencer, J. Park and W. R. Dichtel, Science, 2011, 332, 228-231.

118 M. J. Bojdys, J. Jeromenok, A. Thomas and M. Antonietti, Adv. Mater., 2010, 22, 2202-2205. 
119 S. Ren, M. J. Bojdys, R. Dawson, A. Laybourn, Y. Z. Khimyak, D. J. Adams and A. I. Cooper, Adv. Mater., 2012, 24, 2357-2361.

120 Y. Zhao, K. X. Yao, B. Teng, T. Zhang and Y. Han, Energy Environ. Sci., 2013, 6, 3684.

121 L. Hao, J. Ning, B. Luo, B. Wang, Y. Zhang, Z. Tang, J. Yang, A. Thomas and L. Zhi, J. Am. Chem. Soc., 2015, 137, 219-225.

122 M. G. Rabbani, A. K. Sekizkardes, Z. Kahveci, T. E. Reich, R. Ding and H. M. El-Kaderi, Chem. - Eur. J., 2013, 19, 3324-3328.

123 S. Yan, X. Guan, H. Li, D. Li, M. Xue, Y. Yan, V. Valtchev, S. Qiu and Q. Fang, J. Am. Chem. Soc., 2019, 141, 2920-2924.

124 P. Das and S. K. Mandal, ACS Appl. Mater. Interfaces, 2021, 13, 14160-14168.

125 P. Das, G. Chakraborty, S. Tyagi and S. K. Mandal, ACS Appl. Mater. Interfaces, 2020, 12, 52527-52537.

126 Y.-F. Xie, S.-Y. Ding, J.-M. Liu, W. Wang and Q.-Y. Zheng, J. Mater. Chem. C, 2015, 3, 10066-10069.

127 J.-Y. Yue, X.-H. Liu, B. Sun and D. Wang, Chem. Commun., 2015, 51, 14318-14321.

128 R. Gomes, P. Bhanja and A. Bhaumik, Chem. Commun., 2015, 51, 10050-10053.

129 S.-Y. Ding, X.-H. Cui, J. Feng, G. Lu and W. Wang, Chem. Commun., 2017, 53, 11956-11959.

130 T. Kundu, J. Wang, Y. Cheng, Y. Du, Y. Qian, G. Liu and D. Zhao, Dalton Trans., 2018, 47, 13824-13829.

131 C. Qian, W. Zhou, J. Qiao, D. Wang, X. Li, W. L. Teo, X. Shi, H. Wu, J. Di, H. Wang, G. Liu, L. Gu, J. Liu, L. Feng, Y. Liu, S. Y. Quek, K. P. Loh and Y. Zhao, J. Am. Chem. Soc., 2020, 142, 18138-18149.

132 X. Wu, Y. Hong, B. Xu, Y. Nishiyama, W. Jiang, J. Zhu, G. Zhang, S. Kitagawa and S. Horike, J. Am. Chem. Soc., 2020, 142, 14357-14364.

133 K. Gottschling, G. Savasci, H. Vignolo-González, S. Schmidt, P. Mauker, T. Banerjee, P. Rovó, C. Ochsenfeld and B. V. Lotsch, J. Am. Chem. Soc., 2020, 142, 12146-12156.

134 D.-M. Li, S.-Y. Zhang, J.-Y. Wan, W.-Q. Zhang, Y.-L. Yan, X.-H. Tang, S.-R. Zheng, S.-L. Cai and W.-G. Zhang, CrystEngComm, 2021, 23, 3594-3601.

135 Y. Lu, Y. Liang, Y. Zhao, M. Xia, X. Liu, T. Shen, L. Feng, N. Yuan and Q. Chen, ACS Appl. Mater. Interfaces, 2021, 13, 1644-1650.

136 Y. Li, C. Wang, S. Ma, H. Zhang, J. Ou, Y. Wei and M. Ye, ACS Appl. Mater. Interfaces, 2019, 11, 11706-11714.

137 Z. Li, N. Huang, K. H. Lee, Y. Feng, S. Tao, Q. Jiang, Y. Nagao, S. Irle and D. Jiang, J. Am. Chem. Soc., 2018, 140, 12374-12377.

138 S. Jiang, L. Meng, W. Ma, G. Pan, W. Zhang, Y. Zou, L. Liu, B. Xu and W. Tian, Mater. Chem. Front., 2021, 5, 4193-4201.

139 K. Gottschling, L. Stegbauer, G. Savasci, N. A. Prisco, Z. J. Berkson, C. Ochsenfeld, B. F. Chmelka and B. V. Lotsch, Chem. Mater., 2019, 31, 1946-1955.

140 G. J. Chen, X. B. Li, C. C. Zhao, H. C. Ma, J. L. Kan, Y. Bin Xin, C. X. Chen and Y. Bin Dong, Inorg. Chem., 2018, 57, 2678-2685.

141 G. Chen, H. H. Lan, S. L. Cai, B. Sun, X. Le Li, Z. H. He, S. R. Zheng, J. Fan, Y. Liu and W. G. Zhang, ACS Appl. Mater. Interfaces, 2019, 11, 12830-12837.
142 H. Zhao, Z. Jin, H. Su, X. Jing, F. Sun and G. Zhu, Chem. Commun., 2011, 47, 6389.

143 C. R. DeBlase, K. E. Silberstein, T.-T. Truong, H. D. Abruña and W. R. Dichtel, J. Am. Chem. Soc., 2013, 135, 16821-16824.

144 Y. Zhang, X. Shen, X. Feng, H. Xia, Y. Mu and X. Liu, Chem. Commun., 2016, 52, 11088-11091.

145 D. Kaleeswaran, P. Vishnoi and R. Murugavel, J. Mater. Chem. C, 2015, 3, 7159-7171.

146 M. S. Lohse, T. Stassin, G. Naudin, S. Wuttke, R. Ameloot, D. De Vos, D. D. Medina and T. Bein, Chem. Mater., 2016, 28, 626-631.

147 P. Pachfule, A. Acharjya, J. Roeser, T. Langenhahn, M. Schwarze, R. Schomäcker, A. Thomas and J. Schmidt, J. Am. Chem. Soc., 2018, 140, 1423-1427.

148 M. Zhang, L. Li, Q. Lin, M. Tang, Y. Wu and C. Ke, J. Am. Chem. Soc., 2019, 141, 5154-5158.

149 G. Das, B. P. Biswal, S. Kandambeth, V. Venkatesh, G. Kaur, M. Addicoat, T. Heine, S. Verma and R. Banerjee, Chem. Sci., 2015, 6, 3931-3939.

150 Y. Liu, Y. Wang, H. Li, X. Guan, L. Zhu, M. Xue, Y. Yan, V. Valtchev, S. Qiu and Q. Fang, Chem. Sci., 2019, 10, 10815-10820.

151 J. Guo, Y. Xu, S. Jin, L. Chen, T. Kaji, Y. Honsho, M. A. Addicoat, J. Kim, A. Saeki, H. Ihee, S. Seki, S. Irle, M. Hiramoto, J. Gao and D. Jiang, Nat. Commun., 2013, 4, 2736.

152 S. Dalapati, S. Jin, J. Gao, Y. Xu, A. Nagai and D. Jiang, J. Am. Chem. Soc., 2013, 135, 17310-17313.

153 Z. Li, X. Feng, Y. Zou, Y. Zhang, H. Xia, X. Liu and Y. Mu, Chem. Commun., 2014, 50, 13825-13828.

154 P. Das, G. Chakraborty and S. K. Mandal, ACS Appl. Mater. Interfaces, 2020, 12, 10224-10232.

155 A. Nagai, X. Chen, X. Feng, X. Ding, Z. Guo and D. Jiang, Angew. Chem., Int. Ed., 2013, 52, 3770-3774.

156 Q. Fang, Z. Zhuang, S. Gu, R. B. Kaspar, J. Zheng, J. Wang, S. Qiu and Y. Yan, Nat. Commun., 2014, 5, 4503.

157 K. M. Gupta, K. Zhang and J. Jiang, Ind. Eng. Chem. Res., 2018, 57, 6477-6482.

158 X. Zhuang, W. Zhao, F. Zhang, Y. Cao, F. Liu, S. Bi and X. Feng, Polym. Chem., 2016, 7, 4176-4181.

159 S. Wei, F. Zhang, W. Zhang, P. Qiang, K. Yu, X. Fu, D. Wu, S. Bi and F. Zhang, J. Am. Chem. Soc., 2019, 141, 14272-14279.

160 H. Lyu, C. S. Diercks, C. Zhu and O. M. Yaghi, J. Am. Chem. Soc., 2019, 141, 6848-6852.

161 J. Xu, Y. He, S. Bi, M. Wang, P. Yang, D. Wu, J. Wang and F. Zhang, Angew. Chem., Int. Ed., 2019, 58, 12065-12069.

162 B. C. Patra, S. Khilari, L. Satyanarayana, D. Pradhan and A. Bhaumik, Chem. Commun., 2016, 52, 7592-7595.

163 S. Kim and H. C. Choi, Commun. Chem., 2019, 2, 60.

164 A. F. M. El-Mahdy, Y. Hung, T. H. Mansoure, H. Yu, T. Chen and S. Kuo, Chem. - Asian J., 2019, 14, 1429-1435.

165 J. Roeser, D. Prill, M. J. Bojdys, P. Fayon, A. Trewin, A. N. Fitch, M. U. Schmidt and A. Thomas, Nat. Chem., 2017, 9, 977-982.

166 G. Das, T. Skorjanc, S. K. Sharma, F. Gándara, M. Lusi, D. S. Shankar Rao, S. Vimala, S. Krishna Prasad, J. Raya, D. S. Han, R. Jagannathan, J.-C. Olsen and A. Trabolsi, J. Am. Chem. Soc., 2017, 139, 9558-9565. 
167 G. Das, T. Skorjanc, T. Prakasam, S. Nuryyeva, J.-C. Olsen and A. Trabolsi, RSC Adv., 2017, 7, 3594-3598.

168 T. Skorjanc, D. Shetty, F. Gándara, L. Ali, J. Raya, G. Das, M. A. Olson and A. Trabolsi, Chem. Sci., 2020, 11, 845-850.

169 T. Skorjanc, D. Shetty, S. K. Sharma, J. Raya, H. Traboulsi, D. S. Han, J. Lalla, R. Newlon, R. Jagannathan, S. Kirmizialtin, J.-C. Olsen and A. Trabolsi, Chem. - Eur. J., 2018, 24, 8648-8655.

170 F. Haase, E. Troschke, G. Savasci, T. Banerjee, V. Duppel, S. Dörfler, M. M. J. Grundei, A. M. Burow, C. Ochsenfeld, S. Kaskel and B. V. Lotsch, Nat. Commun., 2018, 9, 2600.

171 M. Lu, M. Zhang, C.-G. Liu, J. Liu, L. Shang, M. Wang, J. Chang, S. Li and Y. Lan, Angew. Chem., Int. Ed., 2021, 60, 4864-4871.

172 J. Liu, T. Yang, Z.-P. Wang, P.-L. Wang, J. Feng, S.-Y. Ding and W. Wang, J. Am. Chem. Soc., 2020, 142, 20956-20961.

173 B. J. Smith, N. Hwang, A. D. Chavez, J. L. Novotney and W. R. Dichtel, Chem. Commun., 2015, 51, 7532-7535.

174 M. Calik, T. Sick, M. Dogru, M. Döblinger, S. Datz, H. Budde, A. Hart Schuh, F. Auras and T. Bein, J. Am. Chem. Soc., 2016, 138, 1234-1239.

175 B. J. Smith, L. R. Parent, A. C. Overholts, P. A. Beaucage, R. P. Bisbey, A. D. Chavez, N. Hwang, C. Parl, A. M. Evans, N. C. Gianneschi and W. R. Dichtel, J. Am. Chem. Soc., 2016, 138, 1234-1239.

176 A. M. Evans, L. R. Parent, N. C. Flanders, R. P. Bisbey, E. Vitaku, M. S. Kirschner, R. D. Schaller, L. X. Chen, N. C. Gianneschi and W. R. Dichtel, Science, 2018, 361, 52-57.

177 B. J. Smith, A. C. Overholts, N. Hwang and W. R. Dichtel, Chem. Commun., 2016, 52, 3690-3693.

178 W. Zhao, J. Qiao, T. Ning and X. Liu, Chin. J. Polym. Sci., 2018, 36, 1-7.

179 D. Zhu, L. B. Alemany, W. Guo and R. Verduzo, Polym. Chem., 2020, 11, 4464-4468.

180 W. Liu, X. Li, C. Wang, H. Pan, W. Liu, K. Wang, Q. Zeng, R. Wang and J. Jiang, J. Am. Chem. Soc., 2019, 141, 17431-17440.

181 S. T. Emmerling, L. S. Germann, P. A. Julien, J. Moudra, M. Etter, T. Friščić, R. E. Dinnebier and B. V. Lotsch, Chem, 2021, 7, 1639-1652.

182 C. H. Feriante, S. Jhulki, A. M. Evans, R. R. Dasari, K. Slicker, W. R. Dichtel and S. R. Marder, Adv. Mater., 2020, 32, 1905776.

183 Z. Zha, L. Xu, Z. Wang, X. Li, Q. Pan, P. Hu and S. Lei, ACS Appl. Mater. Interfaces, 2015, 7, 17837-17843.

184 P. J. Waller, S. J. Lyle, T. M. Osborn Popp, C. S. Diercks, J. A. Reimer and O. M. Yaghi, J. Am. Chem. Soc., 2016, 138, 15519-15522.

185 M. Herrmann, P. B. Kempa, H. Fietzek, T. Altenburg, A. Polyzoidis, C. G. Piscopo and S. Löbbecke, Chem. Ing. Tech., 2016, 88, 967-970.

186 W. Ma, Q. Zheng, Y. He, G. Li, W. Guo, Z. Lin and L. Zhang, J. Am. Chem. Soc., 2019, 141, 18271-18277.

187 B. Gole, V. Stepanenko, S. Rager, M. Grüne, D. D. Medina, T. Bein, F. Würthner and F. Beuerle, Angew. Chem., Int. Ed., 2018, 57, 846-850.

188 S. Wang, Z. Zhang, H. Zhang, A. G. Rajan, N. Xu, Y. Yang, Y. Zeng, P. Liu, X. Zhang, Q. Mao, Y. He, J. Zhao, B.-G. Li, M. S. Strano and W.-J. Wang, Matter, 2019, 1, 1592-1605.
189 S. He, T. Zeng, S. Wang, H. Niu and Y. Cai, ACS Appl. Mater. Interfaces, 2017, 9, 2959-2965.

190 Z. Lu, Y. Liu, X. Liu, S. Lu, Y. Li, S. Yang, Y. Qin, L. Zheng and H. Zhang, J. Mater. Chem. B, 2019, 7, 1469-1474.

191 X. H. Xiong, Z. W. Yu, L. Le Gong, Y. Tao, Z. Gao, L. Wang, W. H. Yin, L. X. Yang and F. Luo, Adv. Sci., 2019, 6, 1900547.

192 D. B. Shinde, H. B. Aiyappa, M. Bhadra, B. P. Biswal, P. Wadge, S. Kandambeth, B. Garai, T. Kundu, S. Kurungot and R. Banerjee, J. Am. Chem. Soc., 2016, 4, 2682-2690.

193 P. Pachfule, S. Kandmabeth, A. Mallick and R. Banerjee, Chem. Commun., 2015, 51, 11717-11720.

194 J. Tan, S. Namuangruk, W. Kong, N. Kungwan, J. Guo and C. Wang, Angew. Chem., Int. Ed., 2016, 55, 13979-13984.

195 Y. Liu, Y. Ma, Y. Zhao, X. Sun, F. Gandara, H. Furukawa, Z. Liu, H. Zhu, C. Zhu, K. Suenaga, P. Oleynikov, A. S. Alshammari, X. Zhang, O. Terasaki and O. M. Yaghi, Science, 2016, 351, 365-369.

196 M. Thommes, K. Kaneko, A. V. Neimark, J. P. Olivier, F. Rodriguez-Reinoso, J. Rouquerol and K. S. W. Sing, Pure Appl. Chem., 2015, 87, 1051-1069.

197 L. A. Baldwin, J. W. Crowe, D. A. Pyles and P. L. McGrier, J. Am. Chem. Soc., 2016, 138, 15134-15137.

198 R. W. Tilford, S. J. Mugavero, P. J. Pellechia and J. J. Lavigne, Adv. Mater., 2008, 20, 2741-2746.

199 Y. Yamaguchi, Y. Matsubara, T. Ochi, T. Wakamiya and Z. Yoshida, J. Am. Chem. Soc., 2008, 130, 16442.

200 Y. Zhao, H. Liu, C. Wu, Z. Zhang, Q. Pan, F. Hu, R. Wang, P. Li, X. Huang and Z. Li, Angew. Chem., Int. Ed., 2019, 58, 5376-5381.

201 M. R. Rao, Y. Fang, S. De Feyter and D. F. Perepichka, J. Am. Chem. Soc., 2017, 139, 2421-2427.

202 E. Jin, J. Li, K. Geng, Q. Jiang, H. Xu, Q. Xu and D. Jiang, Nat. Commun., 2018, 9, 4143.

203 Y. Hong, J. W. Y. Lam and B. Z. Tang, Chem. Soc. Rev., 2011, 40, 5361.

204 J. Mei, Y. Hong, J. W. Y. Lam, A. Qin, Y. Tang and B. Z. Tang, Adv. Mater., 2014, 26, 5429-5479.

205 Z. Chi, X. Zhang, B. Xu, X. Zhou, C. Ma, Y. Zhang, S. Liu and J. Xu, Chem. Soc. Rev., 2012, 41, 3878-3896.

206 H. Ding, J. Li, G. Xie, G. Lin, R. Chen, Z. Peng, C. Yang, B. Wang, J. Sun and C. Wang, Nat. Commun., 2018, 9, 5234.

207 Z.-F. Pang, S.-Q. Xu, T.-Y. Zhou, R.-R. Liang, T.-G. Zhan and X. Zhao, J. Am. Chem. Soc., 2016, 138, 4710-4713.

208 Y. Tian, S.-Q. Xu, C. Qian, Z.-F. Pang, G.-F. Jiang and X. Zhao, Chem. Commun., 2016, 52, 11704-11707.

209 J. Dong, X. Li, S. B. Peh, Y. Di Yuan, Y. Wang, D. Ji, S. Peng, G. Liu, S. Ying, D. Yuan, J. Jiang, S. Ramakrishna and D. Zhao, Chem. Mater., 2019, 31, 146-160.

210 Z.-F. Pang, T.-Y. Zhou, R.-R. Liang, Q.-Y. Qi and X. Zhao, Chem. Sci., 2017, 8, 3866-3870.

211 S. Wan, J. Guo, J. Kim, H. Ihee and D. Jiang, Angew. Chem., Int. Ed., 2008, 47, 8826-8830.

212 J. W. Crowe, L. A. Baldwin and P. L. McGrier, J. Am. Chem. Soc., 2016, 138, 10120-10123.

213 L. Stegbauer, S. Zech, G. Savasci, T. Banerjee, F. Podjaski, K. Schwinghammer, C. Ochsenfeld and B. V. Lotsch, Adv. Energy Mater., 2018, 8, 1703278. 
214 M. G. Mohamed, C.-C. Lee, A. F. M. EL-Mahdy, J. Lüder, M.-H. Yu, Z. Li, Z. Zhu, C.-C. Chueh and S.-W. Kuo, J. Mater. Chem. A, 2020, 8, 11448-11459.

215 Z. Li, S. Han, C. Li, P. Shao, H. Xia, H. Li, X. Chen, X. Feng and X. Liu, J. Mater. Chem. A, 2020, 8, 8706-8715.

216 L. Ascherl, E. W. Evans, J. Gorman, S. Orsborne, D. Bessinger, T. Bein, R. H. Friend and F. Auras, J. Am. Chem. Soc., 2019, 141, 15693-15699.

217 A. Weller, Naturwissenschaften, 1955, 42, 175-176.

218 L. Chen, P. Fu, H. Wang and M. Pan, Adv. Opt. Mater., 2021, 2001952.

219 R. Ali, R. C. Gupta, S. K. Dwivedi and A. Misra, New J. Chem., 2018, 42, 11746-11754.

220 B. P. R. Aradhyula, R. V. Ranga Naidu Chinta, K. Dhanunjayarao and K. Venkatasubbaiah, RSC Adv., 2020, 10, 13149-13154.

221 K. Sakai, S. Takahashi, A. Kobayashi, T. Akutagawa, T. Nakamura, M. Dosen, M. Kato and U. Nagashima, Dalton Trans., 2010, 39, 1989.

222 H.-L. Qian, C. Dai, C.-X. Yang and X.-P. Yan, ACS Appl. Mater. Interfaces, 2017, 9, 24999-25005.

223 H.-Q. Yin, F. Yin and X.-B. Yin, Chem. Sci., 2019, 10, 11103-11109.

224 S. Haldar, D. Chakraborty, B. Roy, G. Banappanavar, K. Rinku, D. Mullangi, P. Hazra, D. Kabra and R. Vaidhyanathan, J. Am. Chem. Soc., 2018, 140, 13367-13374.

225 X. Li, Q. Gao, J. Wang, Y. Chen, Z.-H. Chen, H.-S. Xu, W. Tang, K. Leng, G.-H. Ning, J. Wu, Q.-H. Xu, S. Y. Quek, Y. Lu and K. P. Loh, Nat. Commun., 2018, 9, 2335.

226 X. Guo, T. Mao, Z. Wang, P. Cheng, Y. Chen, S. Ma and Z. Zhang, ACS Cent. Sci., 2020, 6, 787-794.

227 J. R. Lakowicz, Principles of Fluorescence Spectroscopy, Springer, New York, USA, 3rd edn, 2006.

228 R. Gomes and A. Bhaumik, RSC Adv., 2016, 6, 28047-28054.

229 J. F. Wyman, M. P. Serve, D. W. Hobson, L. H. Lee and D. "E” Uddin, J. Toxicol. Environ. Health, 1992, 37, 313-327.

230 A. Dutta, A. Singh, X. Wang, A. Kumar and J. Liu, CrystEngComm, 2020, 22, 7736-7781.

231 M. S. Lohse and T. Bein, Adv. Funct. Mater., 2018, 28, 1705553.

232 W. Zhang, L.-G. Qiu, Y.-P. Yuan, A.-J. Xie, Y.-H. Shen and J.-F. Zhu, J. Hazard. Mater., 2012, 221-222, 147-154.

233 C. Zhang, S. Zhang, Y. Yan, F. Xia, A. Huang and Y. Xian, ACS Appl. Mater. Interfaces, 2017, 9, 13415-13421.

234 L. Chen, L. He, F. Ma, W. Liu, Y. Wang, M. A. Silver, L. Chen, L. Zhu, D. Gui, J. Diwu, Z. Chai and S. Wang, ACS Appl. Mater. Interfaces, 2018, 10, 15364-15368.

235 X. Wu, X. Zhang, Y. Li, B. Wang, Y. Li and L. Chen, J. Mater. Sci., 2021, 56, 2717-2724.

236 Y. Yang, Z. Zhao, Y. Yan, G. Li and C. Hao, New J. Chem., 2019, 43, 9274-9279.

237 Y. Wang, Z. Zhao, G. Li, Y. Yan and C. Hao, J. Mol. Model., 2018, 24, 153.

238 M. Hussain, X. Song, S. Shah and C. Hao, Spectrochim. Acta, Part A, 2020, 224, 117432.

239 F.-Z. Cui, J.-J. Xie, S.-Y. Jiang, S.-X. Gan, D.-L. Ma, R.-R. Liang, G.-F. Jiang and X. Zhao, Chem. Commun., 2019, 55, 4550-4553.

240 Y. Nailwal, A. D. D. Wonanke, M. A. Addicoat and S. K. Pal, Macromolecules, 2021, 53, 6595-6604.
241 Y. Liu, X. Yan, H.-S. Lu, W.-D. Zhang, Y.-X. Shi and Z.-G. Gu, Sens. Actuators, B, 2020, 323, 128708.

242 M. Li, Z. Cui, S. Pang, L. Meng, D. Ma, Y. Li, Z. Shi and S. Feng, J. Mater. Chem. C, 2019, 7, 11919-11925.

243 D. Huang, X. Liu, C. Lai, L. Qin, C. Zhang, H. Yi, G. Zeng, B. Li, R. Deng, S. Liu and Y. Zhang, Microchim. Acta, 2019, 186, 31.

244 J. Zhang, X. Sun and J. Wu, Appl. Sci., 2019, 9, 489.

245 Z. Li, Y. Zhang, H. Xia, Y. Mu and X. Liu, Chem. Commun., 2016, 52, 6613-6616.

246 Y. Cai, Y. Jiang, L. Feng, Y. Hua, H. Liu, C. Fan, M. Yin, S. Li, X. Lv and H. Wang, Anal. Chim. Acta, 2019, 1057, 88-97.

247 Y. Xiong, L. Su, X. He, Z. Duan, Z. Zhang, Z. Chen, W. Xie, D. Zhu and Y. Luo, Sens. Actuators, B, 2017, 253, 384-391.

248 W.-R. Cui, C.-R. Zhang, W. Jiang, R.-P. Liang, S.-H. Wen, D. Peng and J.-D. Qiu, ACS Sustainable Chem. Eng., 2019, 7, 9408-9415.

249 S.-Y. Ding, M. Dong, Y.-W. Wang, Y.-T. Chen, H.-Z. Wang, C.-Y. Su and W. Wang, J. Am. Chem. Soc., 2016, 138, 3031-3037.

250 Y. Zhu, M. Qiao, W. Peng, Y. Li, G. Zhang, F. Zhang, Y. Li and X. Fan, J. Mater. Chem. A, 2017, 5, 9272-9278.

251 Y. Yu, G. Li, J. Liu and D. Yuan, Chem. Eng. J., 2020, 401, 126139.

252 T. Zhang, C. Gao, W. Huang, Y. Chen, Y. Wang and J. Wang, Talanta, 2018, 188, 578-583.

253 L.-L. Wang, C.-X. Yang and X.-P. Yan, Sci. China: Chem., 2018, 61, 1470-1474.

254 P. Asadi, M. Falsafin and M. Dinari, J. Mol. Struct., 2021, 1227, 129546.

255 W.-R. Cui, C.-R. Zhang, W. Jiang, R.-P. Liang and J.-D. Qiu, ACS Appl. Nano Mater., 2019, 2, 5342-5349.

256 W.-R. Cui, C.-R. Zhang, W. Jiang, F.-F. Li, R.-P. Liang, J. Liu and J.-D. Qiu, Nat. Commun., 2020, 11, 436.

257 W. Li, C.-X. Yang and X.-P. Yan, Chem. Commun., 2017, 53, 11469-11471.

258 T. Zhang, N. Ma, A. Ali, Q. Wei, D. Wu and X. Ren, Biosens. Bioelectron., 2018, 119, 176-181.

259 J. Wang, X. Yang, T. Wei, J. Bao, Q. Zhu and Z. Dai, ACS Appl. Bio Mater., 2018, 1, 382-388.

260 P. Wang, F. Zhou, C. Zhang, S.-Y. Yin, L. Teng, L. Chen, X.-X. Hu, H.-W. Liu, X. Yin and X.-B. Zhang, Chem. Sci., 2018, 9, 8402-8408.

261 X. Wu, X. Han, Q. Xu, Y. Liu, C. Yuan, S. Yang, Y. Liu, J. Jiang and Y. Cui, J. Am. Chem. Soc., 2019, 141, 7081-7089.

262 N. Zhao, J.-M. Liu, F.-E. Yang, S.-W. Lv, J. Wang and S. Wang, ACS Appl. Bio Mater., 2021, 4, 995-1002.

263 M. Wang, M. Hu, J. Liu, C. Guo, D. Peng, Q. Jia, L. He, Z. Zhang and M. Du, Biosens. Bioelectron., 2019, 132, 8-16.

264 A. M. Kaczmarek, Y. Liu, M. K. Kaczmarek, H. Liu, F. Artizzu, L. D. Carlos and P. Van Der Voort, Angew. Chem., Int. Ed., 2020, 59, 1932-1940.

265 M. Huang, J. Chong, C. Hu and Y. Yang, Inorg. Chem. Commun., 2020, 119, 108094.

266 A. M. Kaczmarek, H. S. Jena, C. Krishnaraj, H. Rijckaert, S. K. P. Veerapandian, A. Meijerink and P. Van Der Voort, Angew. Chem., Int. Ed., 2021, 60, 3727-3736. 